ORNL/SUB/99-19XSY063V-3

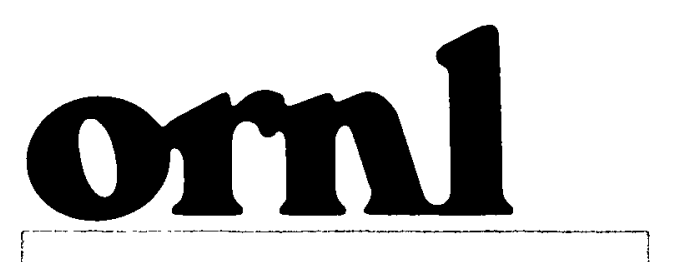

OAK RIDGE NATIONAL LABORATORY

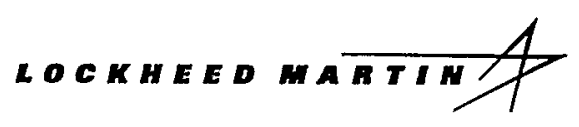

MANAGED AND OPERATED BY LOCKHEED MARTIN ENERGY RESEARCH CORPORATION FOR THE UMTED STATES DEPARTMENT OF ENERGY

ORNL-27 (3-96)

\section{FINAL REPORT}

\section{Evaluation of Mixed MOX-LEU Loading Patterns for LWRs Originating from Inability to Deliver MOX Assemblies: GE BWR/6 Reload Core Study}

Imelda Ariani

Paul J. Turinsky

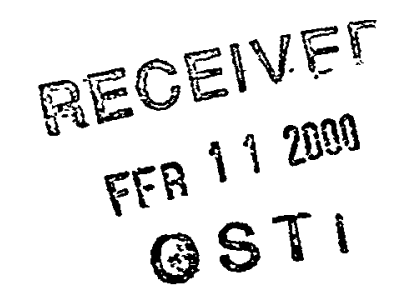


This report was prepared as an account of work sponsored by an agency of the United States Government. Neither the United States Government nor any agency thereof, nor any of their employees, makes any warranty, express or implied, or assumes any legal liability or responsibility for the accuracy, completeness, or usefulness or any information, apparatus, product, or process disclosed, or represents that its use would not infringe privately owned rights. Reference herein to any specific commercial product, process, or service by trade name, trademark, manufacturer, or otherwise, does not necessarily constitute or imply its endorsement, recommendation, or favoring by the United States Government or any agency thereof. The views and opinions of authors expressed herein do not necessarily state or reflect those of the United States Government or any agency thereof. 


\section{DISCLAIMER}

Portions of this document may be illegible in electronic image products. Images are produced from the best available original document. 
FINAL REPORT

\title{
EVALUATION OF MIXED MOX-LEU LOADING PATTERNS FOR LWRs ORIGINATING FROM INABLITY TO DELIVER MOX ASSEMBLIES: GE BWR/6 RELOAD CORE STUDY
}

\author{
Imelda Ariani \\ Paul J. Turinsky
}

Date Published: January 2000

\author{
Prepared by \\ Electric Power Research Center \\ Department of Nuclear Engineering \\ North Carolina State University \\ Raleigh, North Carolina \\ under Subcontract 19XSY063 \\ Prepared for the \\ Computational Physics and Engineering Division \\ OAK RIDGE NATIONAL LABORATORY \\ Oak Ridge, Tennessee 37831 \\ managed by \\ LOCKHEED MARTIN ENERGY RESEARCH CORP. \\ for the \\ U.S. DEPARTMENT OF ENERGY \\ under contract DE-AC05-96OR22464
}


Page Intentionally Blank 


\section{FINAL REPORT}

Evaluation of Mixed MOX-LEU Loading Patterns for LWRs Originating from Inability to Deliver MOX Assemblies

GE BWR/6 Reload Core Study

Imelda Ariani and Paul J. Turinsky

Electric Power Research Center

Department of Nuclear Engineering

North Carolina State University

Raleigh, NC

September, 1999 


\section{Table of Contents}

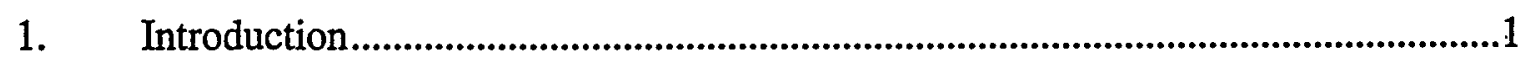

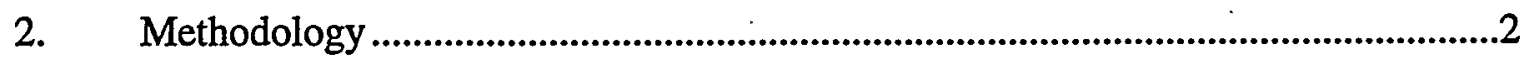

3. Lattice Physics Calculations ..............................................................................2

4. Equilibrium Cycle, Full MOX Core ……………......................................................

5. Initial Mixed MOX-LEU Core........................................................................10

6. Subsequent Mixed MOX-LEU Cores .....................................................................23

6.1 Scenario 1: MOX Feed Assemblies Available for Subsequent Cycles......24

6.2 Transition Back to Full MOX Core ...........................................................27

6.2.1 Scenario 1b Mixed MOX-LEU Core .................................................27

6.2.2 Nearly Full MOX Core .................................................................33

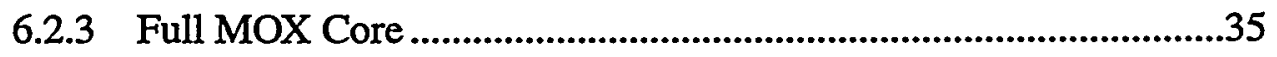

6.3 Summary of Disruption Scenario 1 Cores ..................................................35

6.4 Scenario 2: MOX Feed Batches Continue to be Unavailable for Subsequent Cycle.............................................................................................

6.5 Transition Back to Full LEU Core.............................................................48

6.5.1 Nearly Full LEU Core................................................................48

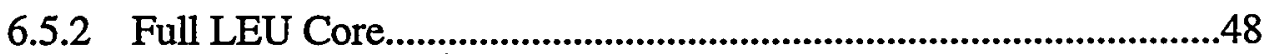

6.6 Summary of Disruption Scenario 2 Cores .................................................53

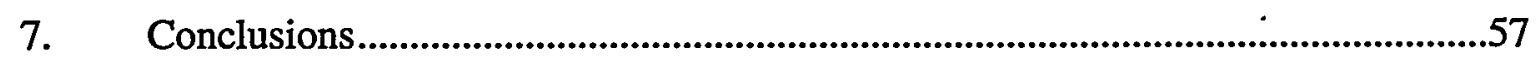

8. Acknowledgment ...................................................................................................57

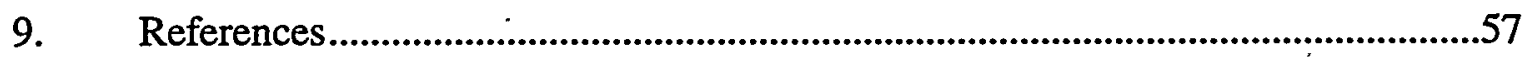

A1. HELIOS Sample Input File...........................................................................63

A2. ZENITH Sample Input/Output File ....................................................................67

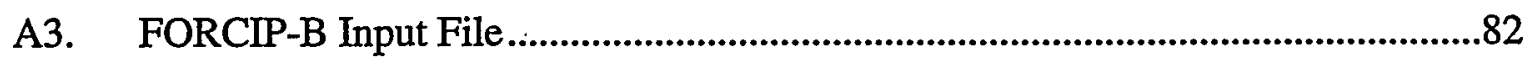

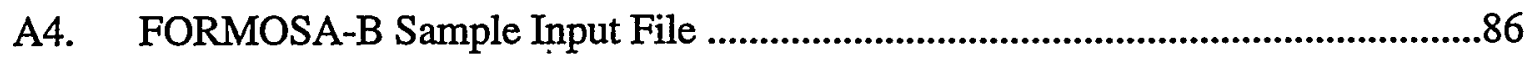

A5. FORMOSA-B Sample Output File .........................................................................98 


\section{List of Figures}

FIGURE 4.1. Process Diagram for Obtaining the Equilibrium Cycle, Full MOX Core........6

FIGURE 4.2. Equilibrium Cycle, Full MOX Core Loading Pattern......................................7

FIGURE 4.3. Equilibrium Cycle, Full MOX Core Shuffle Instructions .................................8

FIGURE 4.4. Equilibrium Cycle, Full MOX Core Control Rod Patterns...............................9

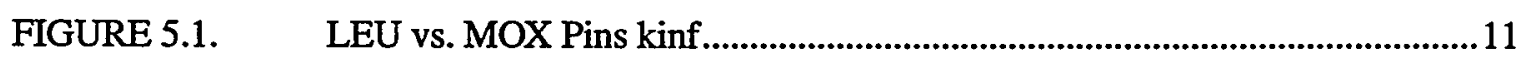

FIGURE 5.2. Gd Loading for MOX Lattices..............................................................13

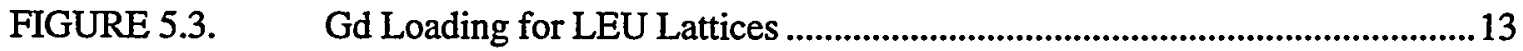

FIGURE 5.4. Lower Lattice LEU vs. MOX Lattice kinf (Uncontrolled, HFP, 0\% Void).... 14

FIGURE 5.5. Lower Lattice LEU vs. MOX Lattice Peak Pin Power (Uncontrolled, HFP, 0\% Void) 14

FIGURE 5.6. LEU Enrichment 1 Fuel Bundle Composition..............................................15

FIGURE 5.7. LEU Enrichment 2 Fuel Bundle Composition ............................................18

FIGURE 5.8. LEU Enrichment 3 Fuel Bundle Composition................................................ 19

FIGURE 5.9. LowerLatticekinfforDifferentLEUEnrichments(Uncontrolled, HFP,0\% Void)

FIGURE 5.10. Initial Mixed Core Control Rod Patterns ......................................................221

FIGURE 5.11. Initial Mixed MOX-LEU Core Loading Pattern .........................................22

FIGURE 6.1. Mixed MOX-LEU Core Disruption Scenarios .............................................23

FIGURE 6.2. Scenario 1 Mixed MOX-LEU Core Loading Pattern ......................................25

FIGURE 6.3. Scenario 1 Mixed MOX-LEU Core Control Rod Patterns ...............................26

FIGURE 6.4. Scenario 1b Mixed MOX-LEU Core Control Rod Patterns ............................28

FIGURE 6.5. Scenario $1 \mathrm{~b}$ (Optimized) Mixed MOX-LEU Core Loading Pattern...............30

FIGURE 6.6. Scenario 1b (Optimized) Mixed MOX-LEU Core Control Rod Patterns.......31

FIGURE 6.7. Scenario 1b Mixed MOX-LEU Core 2D BOC Exposure Map.......................32

FIGURE 6.8. Scenario $1 \mathrm{~b}$ (Optimized) Mixed MOX-LEU Core 2D BOC Exposure Map.32

FIGURE 6.9. Nearly Full MOX Core Control Rod Patterns.................................................34

FIGURE 6.10. Full MOX Core Control Rod Patterns ............................................................36

FIGURE 6.11. Summary of Rated Core Flow for Disruption Scenario 1 Cores ....................37

FIGURE 6.12. Summary of MFLPD for Disruption Scenario 1 Cores ....................................38 
FIGURE 6.13. Summary of MAPRAT for Disruption Scenario 1 Cores ...............................38

FIGURE 6.14. Summary of MFLCPR for Disruption Scenario 1 Cores...............................39

FIGURE 6.15. Summary of Maximum LHGR for Disruption Scenario 1 Cores ...................39

FIGURE 6.16. Summary of MCPR for Disruption Scenario 1 Cores ......................................40

FIGURE 6.17. Summary of keff (ARO, 100\% Flow) for Disruption Scenario 1 Cores........40

FIGURE 6.18. Scenario 2 Mixed MOX-LEU Core Loading Pattern ....................................44

FIGURE 6.19. Scenario 2 Mixed MOX-LEU Core Control Rod Patterns ............................45

FIGURE 6.20. Scenario 2 (Optimized) Mixed MOX-LEU Core Control Rod Patterns........46

FIGURE 6.21. Scenario 2 Mixed MOX-LEU Core 2D BOC Exposure Map........................47

FIGURE 6.22. Scenario 2 (Optimized) Mixed MOX-LEU Core 2D BOC Exposure Map...47

FIGURE 6.23. Nearly Full LEU Core Loading Pattem ......................................................49

FIGURE 6.24. Nearly Full LEU Core Control Rod Patterns ..................................................50

FIGURE 6.25. Full LEU Core Loading Pattern ..................................................................51

FIGURE 6.26. Full LEU Core Control Rod Patterns..............................................................52

FIGURE 6.27. Summary of Rated Core Flow for Disruption Scenario.2 Cores .....................53

FIGURE 6.28. Summary of MFLPD for Disruption Scenario 2 Cores ...................................54

FIGURE 6.29. : Summary of MAPRAT for Disruption Scenario 2 Cores ................................54

FIGURE 6.30. Summary of MFLCPR for Disruption Scenario 2 Cores..................................55

FIGURE 6.31. Summary of Maximum LHGR for Disruption Scenario 2 Cores ...................55

FIGURE 6.32. ' Summary of MCPR for Disruption Scenario 2 Cores ......................................56

FIGURE 6.33. Summary of keff (ARO, 100\% Core Flow) for Disruption Scenario 2 Cores... 


\section{List of Tables}

TABLE 3.1: Instantaneous Branch Case Parameters for HELIOS BWR Lattice Calculations.. 3

TABLE 4.1: GE BWR Core Design and Operating Parameters ....................................................4

TABLE 4.2: $\quad$ Equilibrium Cycle, Full MOX Core's Key Attributes .............................................10

TABLE 5.1: Gd Loading Modifications................................................................................12

TABLE 5.2: Initial Mixed MOX-LEU Core's Key Attributes ..................................................20

TABLE 6.1: Scenario 1 Mixed MOX-LEU Core's Key Attributes............................................24

TABLE 6.2: Scenario 1b Mixed MOX-LEU Core's Key Attributes...........................................27

TABLE 6.3: Scenario 1b (Optimized) Mixed MOX-LEU Core's Key Attributes ......................29

TABLE 6.4: Nearly Full MOX Core's Key Attributes....................................................................33

TABLE 6.5: Full MOX Core's Key Attributes.....................................................................35

TABLE 6.6: Summary of Feed Assemblies' Mean Enrichment for Disruption Scenario 1 Cores

TABLE 6.7: Scenario 2 Mixéd MOX-LEU Core's Key Attributes...........................................44

TABLE 6.8: Scenario 2 (Optimized) Mixed MOX-LEU Core's Key Attributes .......................42

TABLE 6.9: Nearly Full LEU Core's Key Attributes ............................................................43

TABLE 6.10: Full LEU Core's Key Attributes .......................................................................4

TABLE 6.11: Summary of Feed Assemblies' Mean Enrichment for Disruption Scenario 2 Cores 


\section{Introduction}

This study is in support of the weapon's grade Pu disposition project utilizing the reactor option, which entails burning weapon's grade Mixed Oxide (MOX) fuel in Light Water Reactors (LWRs). What is being evaluated is the feasibility of substituting Low Enriched Úranium (LEU) fuel assemblies for weapon's grade MOX assemblies starting from an equilibrium cycle, full MOX core. The specific focus of this report is on the GE Boiling Water Reactor (BWR) core design, starting with an equilibrium cycle, full MOX core identified by GE. The motivation for this study is two fold. Firstly, since there are technical and political uncertainties associated with the utilization of weapon's grade MOX assemblies, there is a potential that some or all of the feed fuel assemblies associated with a reload region may not be available in a timely manner. This condition will be referred to. as the disruption scenario. Secondly, at the conclusion of the $\mathrm{Pu}$ disposition campaign, the reactors involved will need to transition back to full LEU cores. Either condition will result in the insertion of feed LEU assemblies in a core containing once and twice burnt weapon's grade MOX assemblies. There are known design challenges associated with mixed MOX-LEU cores. The emphasis of the current study is noted to be related to the transition from an equilibrium cycle, full MOX core to an equilibrium cycle, full LEU core, with the additional complexity of not knowing when this will occur for the disruption scenario.

The design features of interest include the lattice design, core loading pattern (LP), and control rod pattern (CRP). The lattice design involves the placement of fuel and burnable poison (BP) material within the fuel lattice. The MOX lattice designs used in this study have been restricted to the existing GE designs used in their full MOX core study. The LEU lattice designs were created based upon the MOX lattice designs, as described in Section 3. In determining the LP, most active LP constraints have been imposed, the exception being the cold shutdown margin constraint. The CRP is determined utilizing FORMOSA-B's CRP search capability for conventional cores. 


\section{Methodology}

Core models are established by generating two-group, homogenized cross-sections via the lattice physics code HELIOS [3], and solving the two-group neutron diffusion equation via the core simulator code FORMOSA-B [5]. Linkage codes ZENITH [3] and FORCIP-B [4] are also used to process the cross sections to the form required by FORMOSA-B.

FORMOSA-B is capable of optimizing the fuel loading pattern (LP) for a BWR while simultaneously determining the Control Rod Patterns (CRP). It also differs from FORMOSA-P in the following manners: 3-D, only macroscopic depletion capability, full two-phase flow model with inlet flow redistribution, critical flow search, usage of BWR relevant objectives and constraints, and peak pin-to-box pin reconstruction.

\section{Lattice Physics Calculations}

Lattice physics calculations for MOX fuel lattices were completed by the team from Oak Ridge National Laboratory utilizing HELIOS/ZENITH codes. LEU lattice designs and calculations were completed by the author. The MOX fuel lattice designs were taken from the $\mathrm{UO}_{2}$-alike $\mathrm{MOX}$ fuel bundle designs from the $\mathrm{GE}$ report [6]. Each LEU lattice is designed such that the LEU lattice's $k_{\text {inf }}$ at the end of one cycle of exposure will be approximately the same as the MOX lattice's $k_{\text {inf }}$ it is substituting for. This is done for each MOX lattice design to generate the equivalent LEU lattice design. HELIOS lattice physics calculations were then completed for a range of LEU lattice designs determined by perturbing slightly up and down the fuel pin enrichments for the equivalent LEU lattices, thereby generating an inventory of LEU lattices with slightly different reactivities. This was necessary since FORMOSA-B does not treat the assembly enrichment as a continuous variable since BWR assemblies employ multiple pin enrichments and axially segmented lattice designs, hence the concept of an assembly enrichment is not applicable. Thus, the user must carefully select the feed fuel assembly 
designs to employ or allow FORMOSA-B to select from an inventory of assembly designs the appropriate mix, to satisfy the cycle energy requirements. For both MOX and LEU lattice designs, integral gadolinia burnable absorbers in the $\mathrm{UO}_{2}$ rods are employed. This was done to avoid using integral burnable absorber in MOX pins, a restriction placed on the design by DOE due to lack of burnup experience for such a configuration. For each lattice design, the lattice was depleted to high burnup at base case conditions, i.e. Hot Full Power (HFP) average moderator density and fuel temperature. Multiple instantaneous branch cases were executed during the depletion, enabling characterization of the crosssections as a function of moderator density, fuel temperature, void fraction, and rodded/ unrodded configuration within FORMOSA-B in order to apply spatially dependent, feedback corrections. Specifically for each lattice type, one base and six branch cases calculations were performed (Table 3.1). This was done for three unrodded depletions utilizing different void fractions. In addition, a rodded depletion at each void fraction was completed, with an instantaneous branch to the unrodded state.

TABLE 3.1: Instantaneous Branch Case Parameters for HELIOS BWR Lattice Calculations

\begin{tabular}{|l|c|c|c|c|}
\hline \multicolumn{1}{|c|}{ CASE } & T FUEL $(\mathbf{K})$ & $\begin{array}{c}\text { Void* } \\
\text { Fraction }\end{array}$ & $\begin{array}{c}\text { Rodded } \\
\text { (Y/N) }\end{array}$ & $\begin{array}{c}\text { Power } \\
\text { Level W/g }\end{array}$ \\
\hline \hline BASE & 792.5 & $0 \%$ & $\mathrm{~N}$ & 26.47 \\
\hline BRANCH 1: VOID LOW & 792.5 & $40 \%$ & $\mathrm{~N}$ & 26.47 \\
\hline BRANCH 2: VOID HIGH & 792.5 & $70 \%$ & $\mathrm{~N}$ & 26.47 \\
\hline BRANCH 3: T FUEL DECREASE & 560.0 & $0 \%$ & $\mathrm{~N}$ & 26.47 \\
\hline BRANCH 4: CONTROLLED HFP & 792.5 & $0 \%$ & $\mathrm{Y}$ & 26.47 \\
\hline BRANCH 5: UNCONTROLLED CZP & 293.0 & $0 \%$ & $\mathrm{~N}$ & 0.0 \\
\hline BRANCH 6: CONTROLLED CZP & 293.0 & $0 \%$ & $\mathrm{Y}$ & 0.0 \\
\hline HFP RODDED DEPLETION & 792.5 & $0 \%$ & $\mathrm{Y}$ & 26.47 \\
\hline
\end{tabular}

Note: three void fractions $(0 \%, 40 \%, 70 \%)$ depletions required

The FORMOSA-B Cross Section Interface Program (FORCIP-B) has been modified to read the ZENITH outputs. 


\section{Equilibrium Cycle, Full MOX Core}

Table 4.1 provides the core design and operating parameters assumed throughout this study. A FORMOSA-B model has beien established for an equilibrium cycle, full MOX core loading pattern identified by GE.

TABLE 4.1: GE BWR Core Design and Operating Parameters

\begin{tabular}{|lc|}
\hline \multicolumn{1}{|c|}{ Parameter } & Value \\
\hline \hline Power level & $3833 \mathrm{MWth}$ \\
System pressure & $1047 \mathrm{psia}$ \\
$100 \%$ Core coolant flow rate & $112.5 \mathrm{Mlbm} / \mathrm{hr}$ \\
Coolant inlet subcooling & $21.23 \mathrm{Btu} / \mathrm{bm}$ \\
Fraction of power generated in fuel rods & 0.98 \\
Fraction of power deposited in bypass flow regions & 0.02 \\
Target cycle length & $12,350 \mathrm{MWd} / \mathrm{ST}$ \\
Number of fuel assemblies & 800 \\
Number of feed assemblies & 264 \\
Fuel lattice & $9 \times 9$ \\
Fuel rods per assembly & 74 \\
Active fuel height & 150 in. \\
Core loading & $169.3 \mathrm{ST}$ \\
MLHGR limit & $14.4 \mathrm{~kW} / \mathrm{ft}$ (operating limit) \\
& $12.96 \mathrm{~kW} / \mathrm{ft}$ (design target) \\
MCPR limit & 1.29 (operating limit) \\
& 1.387 (design target) \\
\hline
\end{tabular}

The BWR-6 equilibrium cycle full MOX core was developed utilizing the FORMOSA-B code using the LP and shuffle instructions (Figure 4.2 and Figure 4.3, respectively) provided by GE. Note that the LP in Figure 4.2 is different from the LP in Figure 1.1.5-1 of the GE Report [6], since GE could not locate the shuffle instructions for 
the LP presented in the GE Report. The process involved in obtaining the equilibrium cycle full MOX core is shown in Figure 4.1. For the initial guess, the bundle burnup values for all axial nodes within the bundle were set to the following values: $12350 \mathrm{MWd} / \mathrm{ST}$, $24700 \mathrm{MWd} / \mathrm{ST}$, and $37050 \mathrm{MWd} / \mathrm{ST}$ for once-, twice, and thrice-burnt assemblies, respectively. Utilizing the multicycle restart and the CRP search capabilities for a Conventional Core in FORMOSA-B, we iterated the cycle depletions and CRP optimizations until convergence was achieved. The control rod sequence exchanges are performed at the end of each $1.5 \mathrm{GWd} / \mathrm{ST}$ depletion. The sequence chosen follows the control rod exchange sequence presented in the GE Report, i.e. $A 2 \rightarrow B 1 \rightarrow A 1 \rightarrow B 2 \rightarrow A 2 \rightarrow e t c$. During depletions, the core flow rates are adjusted to meet the target $k_{\text {eff }}$. The target $k_{\text {eff }}$ is kept at 1.000 throughout the cycle since bias values applicable to FORMOSA:B could not be developed based upon the data available.

The equilibrium cycle, full MOX core results are presented in Figure 4.4 and Table 4.2. The Critical Power Ratio (CPR) predictions are based upon the GEXI correlation applicable to GE-11 bundles, which was provided to us by GE and incorporated into the FORMOSA-B code. The R-factor data required for GEXL calculations are assumed to be independent of notch insertions.

Table 4.2 indicates that the core flows required to achieve criticality are acceptable, utilizing low flows earlier in the cycle to maximize spectral shift production of plutonium and higher flows later in the cycle to retain criticality. Table 4.2 also presents the thermal limits (MFLPD: Maximum Fraction of Linear Power Density, MAPRAT: maximum fraction of average linear power density, MFLCPR: Maximum Fraction of Limiting Critical Power Ratio, Max. LHGR: Maximum Linear Heat Generation Rate, and MCPR), where it is to be noted that all thermal margins are less than or about 0.95 . The value of the thermal margins are comparable to the values presented in the GE Report. Since we are using a different LP and CRPs, one would not expect them to be exactly the same. We conclude that we have established a feasible full MOX core FORMOSA-B model, which will serve as the basis for subsequent, disruption scenario studies. 


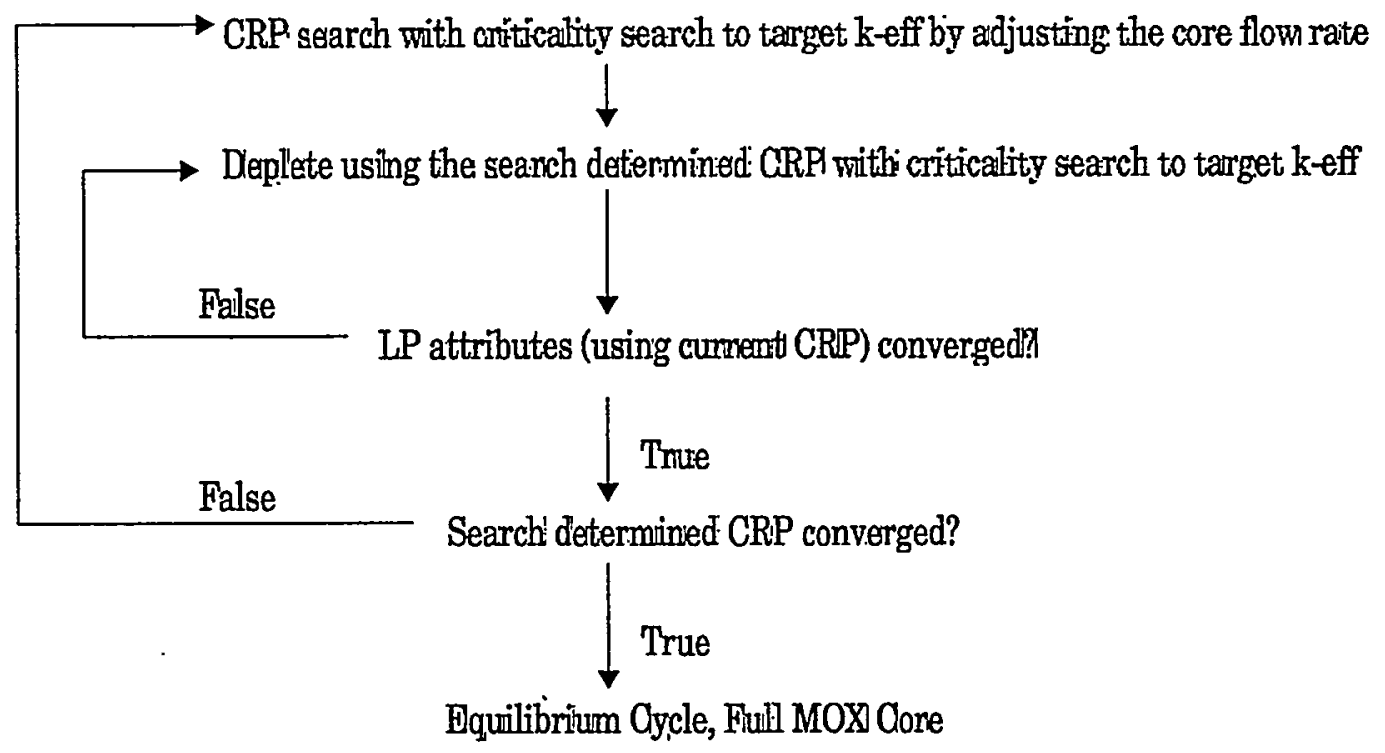

FIGURE 4.1. Process Diagram for Obtaining the Equilibrium Cycle, Full MOX Core 
First Index

\begin{tabular}{|c|c|c|c|c|c|c|c|c|c|c|c|c|c|c|c|c|}
\hline & 16 & 15 & 14 & 13 & 12 & 11 & 10 & 9 & 8 & 7 & 6 & 5 & 4 & 3 & 2 & 1 \\
\hline 16 & 2 & 1 & 1 & 1 & 1 & 1 & 1 & 2 & $\mathrm{~F}$ & 2 & $F$ & 2 & $\mathrm{~F}$ & 1 & $F$ & 2 \\
\hline 15 & .1 & $\mathrm{~F}$ & 2 & $F$ & 2 & F & 2 & $\mathrm{~F}$ & 2 & $\mathrm{~F}$ & 2 & $\mathrm{~F}$ & 2 & $\mathrm{~F}$ & 1 & 3 \\
\hline 14 & 1 & 2 & 1 & 1 & 1 & 1 & 1 & 1 & $\mathrm{~F}$ & 1 & $F$ & 1 & $F$ & 2 & 2 & \\
\hline c $\quad 13$ & 1 & $F$ & 1 & $\mathrm{~F}$ & 2 & $\mathrm{~F}$ & 2 & $\mathrm{~F}$ & 2 & F & 2 & $\mathrm{~F}$ & 2 & 1 & 2 & \\
\hline 12 & 1 & 2 & 1 & 2 & 1 & 1 & 1 & 1 & $F$ & 1 & $F$ & 1 & $F$ & 1 & 2 & \\
\hline 11 & 1 & $F$ & 1 & $F$ & 1 & $\mathrm{~F}$ & 2 & $\mathrm{~F}$ & 2 & $\mathrm{~F}$ & 2 & $\mathrm{~F}$ & 2 & 1 & 2 & \\
\hline 10 & 1 & 2 & 1 & 2 & 1 & 2 & F & 1 & $\mathrm{~F}$ & 1 & $F$ & 1 & $\mathrm{~F}$ & 1 & 2. & \\
\hline 9 & 2 & $F$ & 1 & $F$ & 1 & $\mathrm{~F}$ & 1 & $\mathrm{~F}$ & 2 & $\mathrm{~F}$ & 2 & $\mathrm{~F}$ & 1 & 2 & & \\
\hline 8 & $\mathrm{~F}$ & 2 & F & 2 & $F$ & 2 & $F$ & 2 & $\mathrm{~F}$ & 1 & $F$ & 1 & 2 & & & \\
\hline 7 & 2 & F & 1 & $\mathrm{~F}$ & 1 & $\mathrm{~F}$ & 1 & $F$ & 1 & $\mathrm{~F}$ & 2 & 1 & 2 & & & \\
\hline 6 & $\mathrm{~F}$ & 2 & $F$ & 2 & $\mathrm{~F}$ & 2 & $\mathrm{~F}$ & 2 & $F$ & 2 & $F$ & 1 & 2 & & & \\
\hline 5 & 2 & $\mathrm{~F}$ & 1 & $\mathrm{~F}$ & 1 & $\mathrm{~F}$ & 1 & $F$ & 1 & 1 & 1 & 2 & & & . & \\
\hline 4 & F & 2 & F & 2 & F & 2 & $F$ & 1 & 2 & 2 & 2 & & & & & \\
\hline 3 & 1 & $\mathrm{~F}$ & 2 & 1 & 1 & 1 & 1 & 2 & & & & & & & & \\
\hline 2 & $F$ & 1 & 2 & 2 & 2 & 2 & 2 & & & & & & & . & & \\
\hline $\mathbf{1}$ & 2 & 3 & & & & & & & & & & & & & & \\
\hline
\end{tabular}

The numbers represent the number of previous cycles the bundle has been in the core. The symbol $\mathrm{F}$ denotes fresh fuels.

FIGURE 4.2. Equilibrium Cycle, Full MOX Core Loading Pattern 
First Index

\begin{tabular}{|c|c|c|c|c|c|c|c|c|c|c|c|c|c|c|c|c|}
\hline & 16 & 15 & 14 & 13 & 12 & 11 & 10 & 9 & 8 & 7 & 6 & 5 & 4 . & 3 & 2 & 1 \\
\hline 16 & $\mid 12,12$ & 9,11 & 5,11 & 8,12 & 7,7 & 7,13 & 6,8 & 13,16 & F & 7,8 & F & 5,14 & F & 11,15 & F & 11,12 \\
\hline 15 & 11,9 & F & 5,12 & $\mathbf{F}$ & 15,16 & F & 12,14 & F & 12,16 & $\mathrm{~F}$ & 5,8 & F & 5,7 & F & 3,15 & 6,7 \\
\hline 14 & 11,5 & 12,5 & 13,13 & 7,11 & 4,6 & 9,13 & 4,14 & 8,8 & F & 7,9 & F & 8,16 & F & 3,10 & 13,14 & \\
\hline 13 & 12,8 & $F$ & 11,7 & $\mathrm{~F}$ & 5,10 & $\mathrm{~F}$ & $\mid 10,14$ & F & 14,16 & $\mathrm{~F}$ & 3,16 & F & 4,9 & 5,13 & 7,14 & \\
\hline 12 & 15,15 & 16,15 & 16,4 & 10,5 & 6,6 & 8,14 & 4,12 & 6,12 & F & 7,15 & F & 6,10 & F & 10,10 & 9,14 & \\
\hline 11 & 13,7 & $\mathrm{~F}$ & 13,9 & $\mathbf{F}$ & 14,8 & F & $\mid 10,12$ & $\mathrm{~F}$ & 10,16 & F & 3,13 & F & 2,15 & 5,15 & 7,10 & \\
\hline 10 & 8,6 & $\mid 14,12$ & 14,4 & 14,10 & 12,4 & 12,10 & F & 6,14 & $F$ & 11,13 & $\mathbf{F}$ & 9,15 & F & 4,10 & 9,10 & \\
\hline 9 & 16,13 & F & 11,11 & $F$ & 12,6 & $\mathrm{~F}$ & 14,6 & F & 3,12 & F & 3,11 & F & 5,9 & 7,12 & & \\
\hline 8 & F & 16,12 & F & 16,14 & F & 16,10 & F & 12,3 & F & 6,16 & $\mathrm{~F}$ & 8,10 & 11,16 & & & \\
\hline 7 & 8,7 & F & 9,7 & F & 15,7 & $F$ & 13,11 & F & 16,6 & $\mathbf{F}$ & 5,6 & 13,15 & 11,14 & & & \\
\hline 6 & F & 8,5 & $\mathrm{~F}$ & 16,3 & F & 13,3 & F & 11,3 & F & 6,5 & F & 2,16 & 9,12 & & & \\
\hline 5 & 14,5 & F & 16,8 & $\mathbf{F}$ & 10,6 & F & 15,9 & $F$ & 10,8 & 15,13 & 16,2 & 14,14 & & & & \\
\hline 4 & $\mathrm{~F}$ & 7,5 & F & 9,4 & F & 15,2 & F & 9,5 & $\mid 16,11$ & 14,11 & 12,9 & & & & & \\
\hline 3 & 15,11 & F & 10,3 & 13,5 & 9,9 & 15,5 & 10,4 & 12,7 & & & & & & & & \\
\hline 2 & F & 15,3 & 14,13 & 14,7 & 14,9 & 10,7 & 10,9 & & \multirow{2}{*}{\multicolumn{8}{|c|}{$\begin{array}{l}\text { The two numbers represent the location of the bundle in } \\
\text { the previous cycle. }\end{array}$}} \\
\hline 1 & 12,11 & 7,6 & & & & & & & & & & & & & & \\
\hline
\end{tabular}

FIGURE 4.3. Equilibrium Cycle, Full MOX Core Shuffle Instructions 
0-1.5 GWd/ST

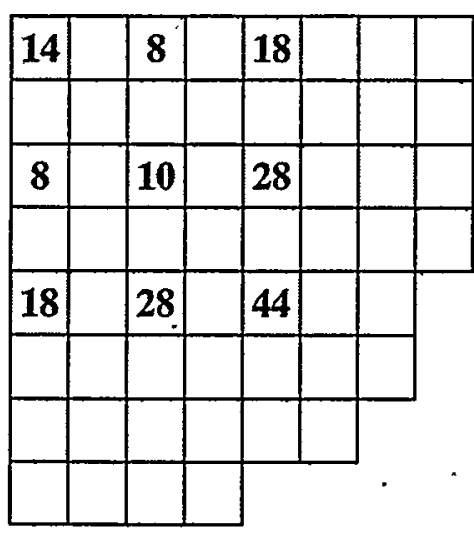

4.5-6.0 GWd/ST

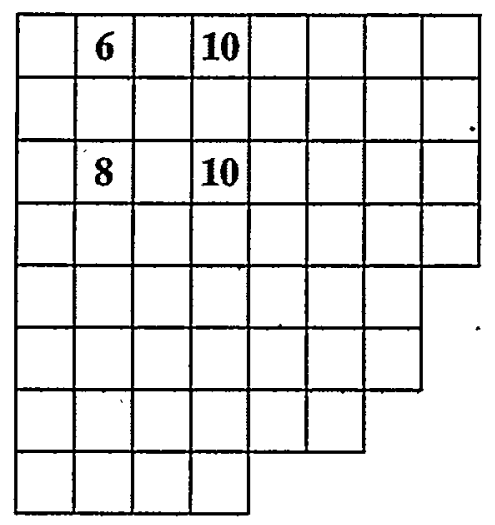

9.0-10.5 GWd/ST
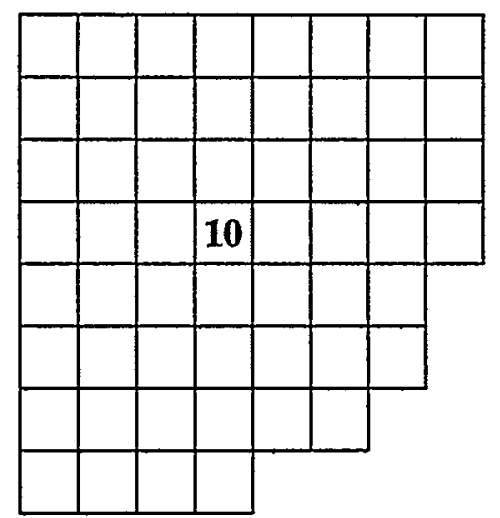

1.5-3.0 GWd/ST

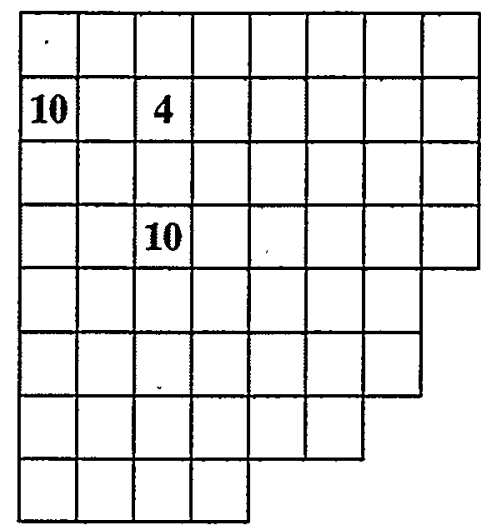

6.0-7.5 GWd/ST

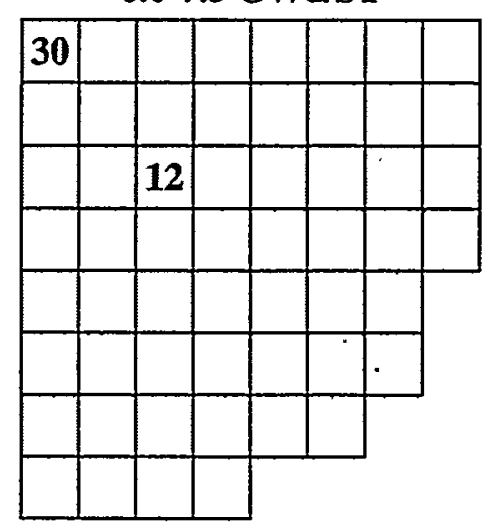

10.5-11.5 GWd/ST

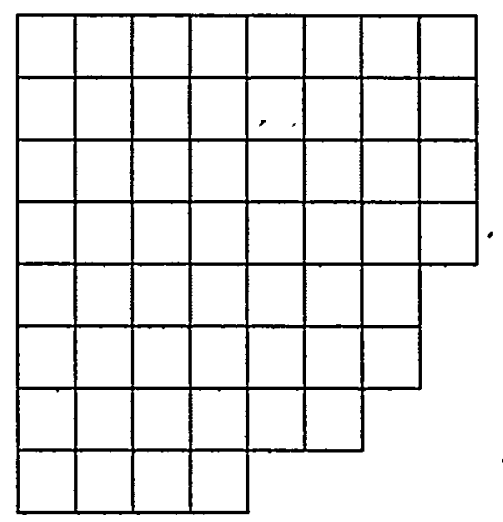

11.5-12.35 GWd/ST

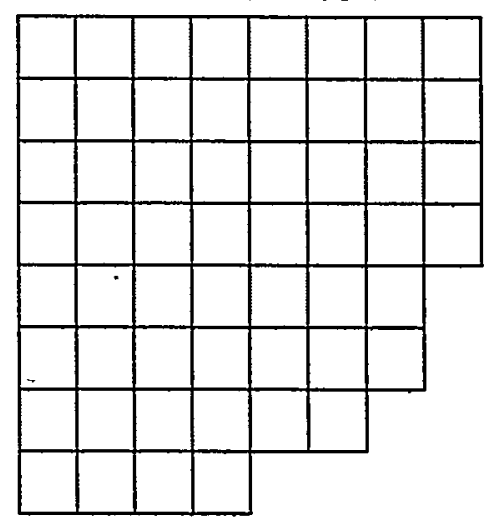

3.0-4.5 GWd/ST

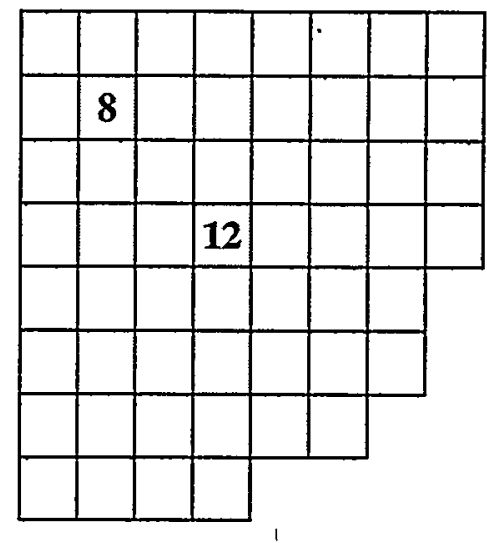

7.5-9.0 GWd/ST.

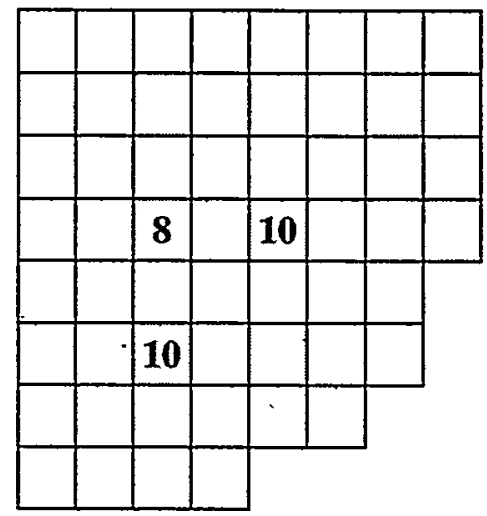


TABLE 4.2: Equilibrium Cycle, Full MOX Core's Key Attributes

\begin{tabular}{|c|ccccccc|}
\hline $\begin{array}{c}\text { Cycle } \\
\text { Exposure } \\
\text { (GWd/ST) }\end{array}$ & $\begin{array}{c}\text { \% Rated } \\
\text { Core } \\
\text { Flow }\end{array}$ & MFLPD & MAPRAT & MFLCPR & $\begin{array}{c}\text { MHGR } \\
\text { kw/ft. }\end{array}$ & MCPR & $\begin{array}{c}\text { 100\% Core } \\
\text { Flow) }\end{array}$ \\
\hline \hline 0 & 83.3422 & $\mathbf{0 . 9 3 4 7}$ & 0.8593 & $\mathbf{0 . 9 3 1 7}$ & 12.11 & 1.49 & 1.0188 \\
1.5 & 83.1626 & 0.8763 & 0.7978 & 0.9057 & 11.36 & 1.53 & 1.0204 \\
3.0 & 83.5822 & 0.9058 & 0.7999 & 0.9195 & 11.74 & 1.51 & 1.0167 \\
4.5 & 93.3264 & 0.8964 & $\mathbf{0 . 8 7 9 3}$ & 0.8689 & 11.62 & 1.60 & 1.0141 \\
6.0 & 84.0028 & 0.8986 & 0.8787 & 0.9020 & 11.65 & 1.54 & 1.0129 \\
7.5 & 94.5321 & 0.9033 & 0.8741 & 0.9298 & 11.71 & 1.49 & 1.0130 \\
9.0 & 86.2311 & 0.8526 & 0.8309 & 0.9326 & 11.05 & 1.49 & 1.0121 \\
10.5 & 86.9833 & 0.8933 & 0.7979 & 0.8933 & 11.58 & 1.56 & 1.0084 \\
11.5 & 94.3653 & 0.8475 & 0.8090 & 0.8753 & 10.98 & 1.59 & 1.0040 \\
12.35 & $\mathbf{1 0 3 . 8 1 4 0}$ & 0.8134 & 0.8396 & 0.8674 & 10.54 & 1.60 & 0.9992 \\
\hline
\end{tabular}

\section{Initial Mixed MOX-LEU Core}

In the first cycle in which the LEU feed assemblies are loaded into the core, all - MOX feed assemblies are assumed to be unavailable and replaced by LEU feed assemblies. This will be referred to as the initial mixed MOX-LEU core. For the initial mixed MOX-LEU core, the following design approach was employed. Since there is no information available from the GE Report or elsewhere regarding the LEU fuel assembly design, we have to design the LEU assemblies ourselves. For each MOX fuel bundle, there are three lattice designs which correspond to three elevation spans: upper, middle, and lower. There are four enrichment types of MOX pins, 2.3, 3.6, 4.0, and 5.4 w/o WGPu, used in the three lattices of the MOX fuel bundle. To approximately determine the ${ }^{235} \mathrm{U}$ 
enrichment of the LEU pins equivalent to the MOX pins, which will be used when replacing the MOX pins in developing the LEU lattices, we developed a lattice which has an equivalent LEU pin enrichment for each of the MOX pin enrichments. We accomplished this equivalence by utilizing HELIOS to obtain the ${ }^{235} \mathrm{U}$ enrichment for a uniform pin-wise enrichment distribution LEU lattice which produces approximately the same $\mathrm{k}_{\mathrm{inf}}$ as the uniform pin-wise enrichment distribution MOX lattice for each of the MOX pin enrichment (at the end of one cycle of exposure, which is $\sim 17 \mathrm{GWd} / \mathrm{MT}$ ). The results are summarized in Figure 5.1.
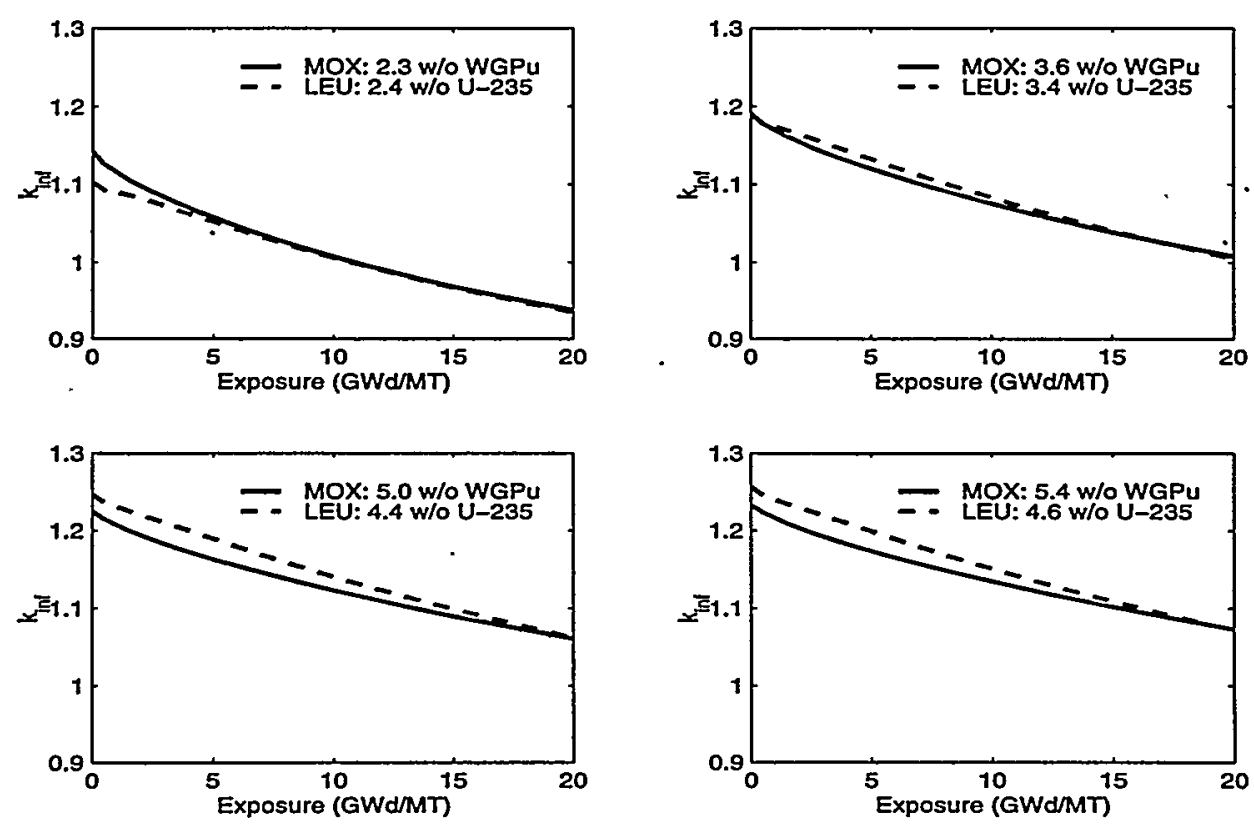

FIGURE 5.1. LEU vs. MOX Pins $k_{\text {inf }}$

The MOX pins in the three MOX lattices are then replaced by their equivalent LEU pins to create the LEU lattices. Similar to the original MOX lattice design, all LEU rods which replace the MOX pins are also axially uniform. Modifications also need to be completed for Gd pins, due to differences in Gd worth and burnout rate in LEU versus MOX lattices. The LEU lattices will need less Gd pins since the gadolinia is worth more in the softer energy spectrum of an LEU versus MOX lattice. There are $16 \mathrm{Gd}$ rods in the 
MOX design (Figure 5.2); whereas, the LEU design producing nearly equivalent reactivity hold-down at BOL was determined to have six less Gd rods (Figure 5.3). Note that the ${ }^{235} \mathrm{U}$ enrichments in the $\mathrm{UO}_{2}$ pins which previously contained gadolinia are kept the same as the original values. Therefore, the enrichment for an LEU pin in locations where Gd pins previously existed is not axially uniform. The gadolinium enrichment in the Gd rods also needs to be increased to accommodate the fact that the LEU lattice burns out the gadolinia faster than the MOX lattice due to the softer energy spectrum. This fact is supported by results in Figure 5.4. Figure 5.5 presents the peak relative pin power versus burnup for the axially lower MOX lattice and various Gd rod designs for the LEU lattices. The Gd loading modifications required to produce approximately the same burnout rate, hence hot excess core reactivity, are summarized in Table 5.1. The resulting lattices that produce equivalent LEU lattices to the MOX lattices will be referred to as enrichment 1 lattices, whose enrichment distributions are shown in Figure 5.6.

TABLE 5.1: Gd Loading Modifications

\begin{tabular}{|c|c|c|c|c|}
\hline \multirow{2}{*}{ Lattice } & \multicolumn{2}{|c|}{ Number of Gd Rods } & \multicolumn{2}{c|}{ Gd w/o } \\
\cline { 2 - 5 } & MOX & LEU & MOX & LEU \\
\hline \hline Upper & 16 & 10 & 3.5 & 4.5 \\
\hline Middle & 16 & 10 & 3.5 & 5.0 \\
\hline Lower & 16 & 10 & 4.5 & 6.5 \\
\hline
\end{tabular}




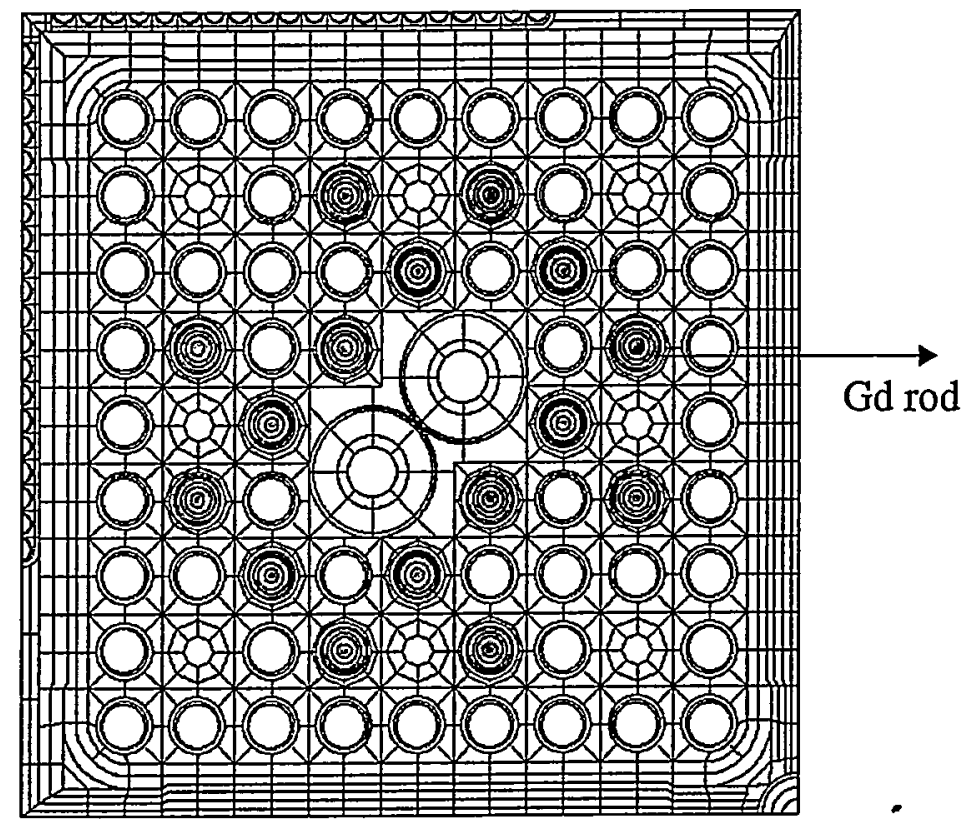

FIGURE 5.2. Gd Loading for MOX Lattices

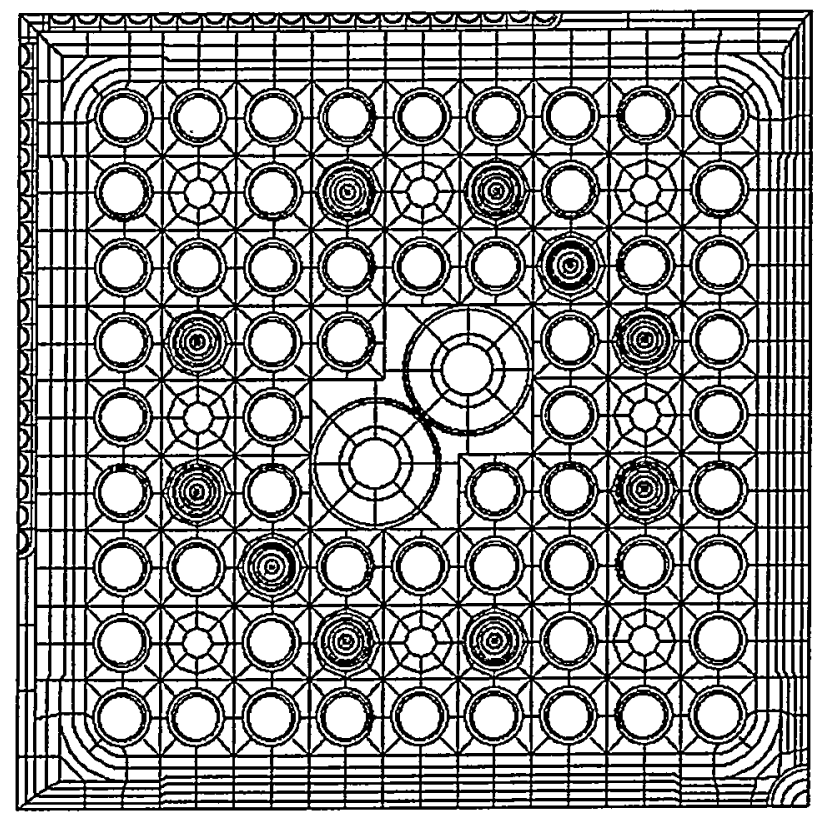

FIGURE 5.3. Gd Loading for LEU Lattices 


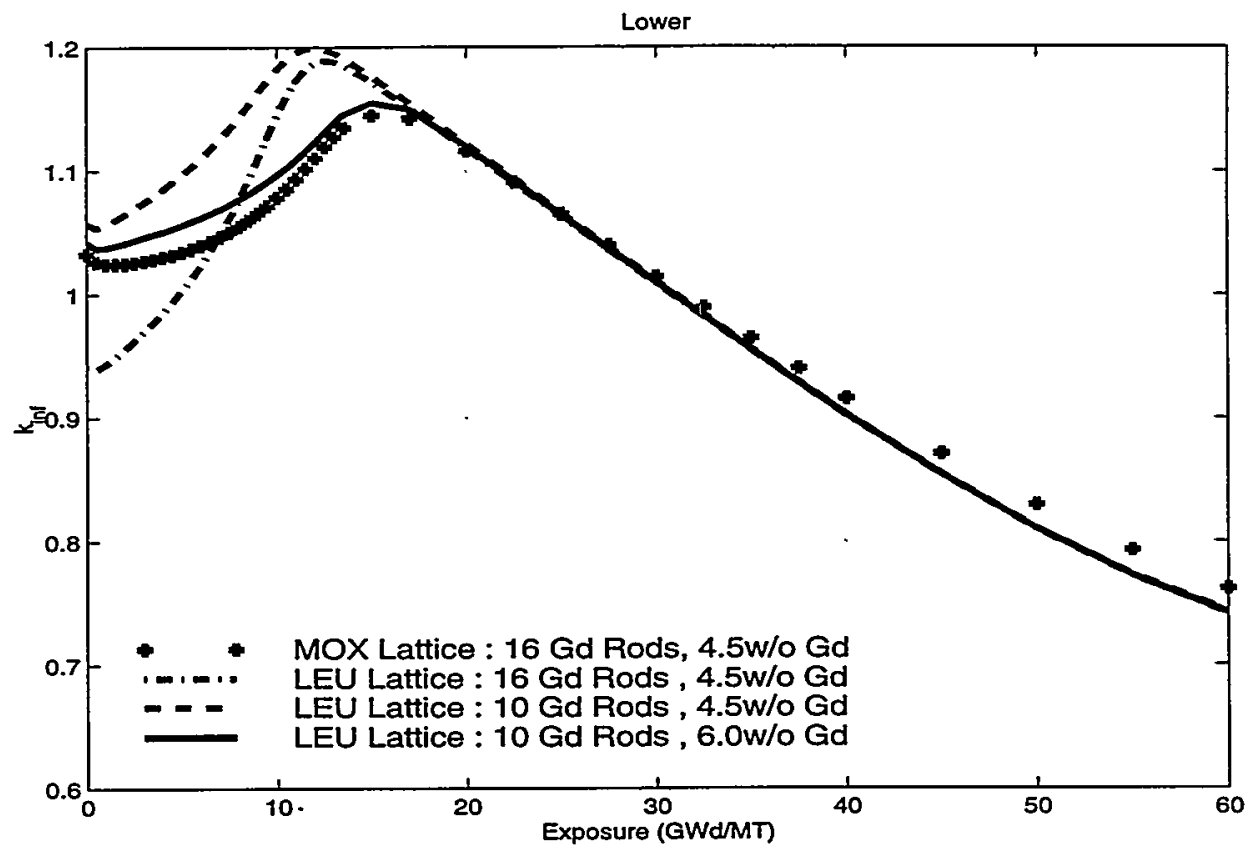

FIGURE 5.4. Lower Lattice LEU vs. MOX Lattice $k_{\text {inf }}$ (Uncontrolled, HFP, $0 \%$ Void)

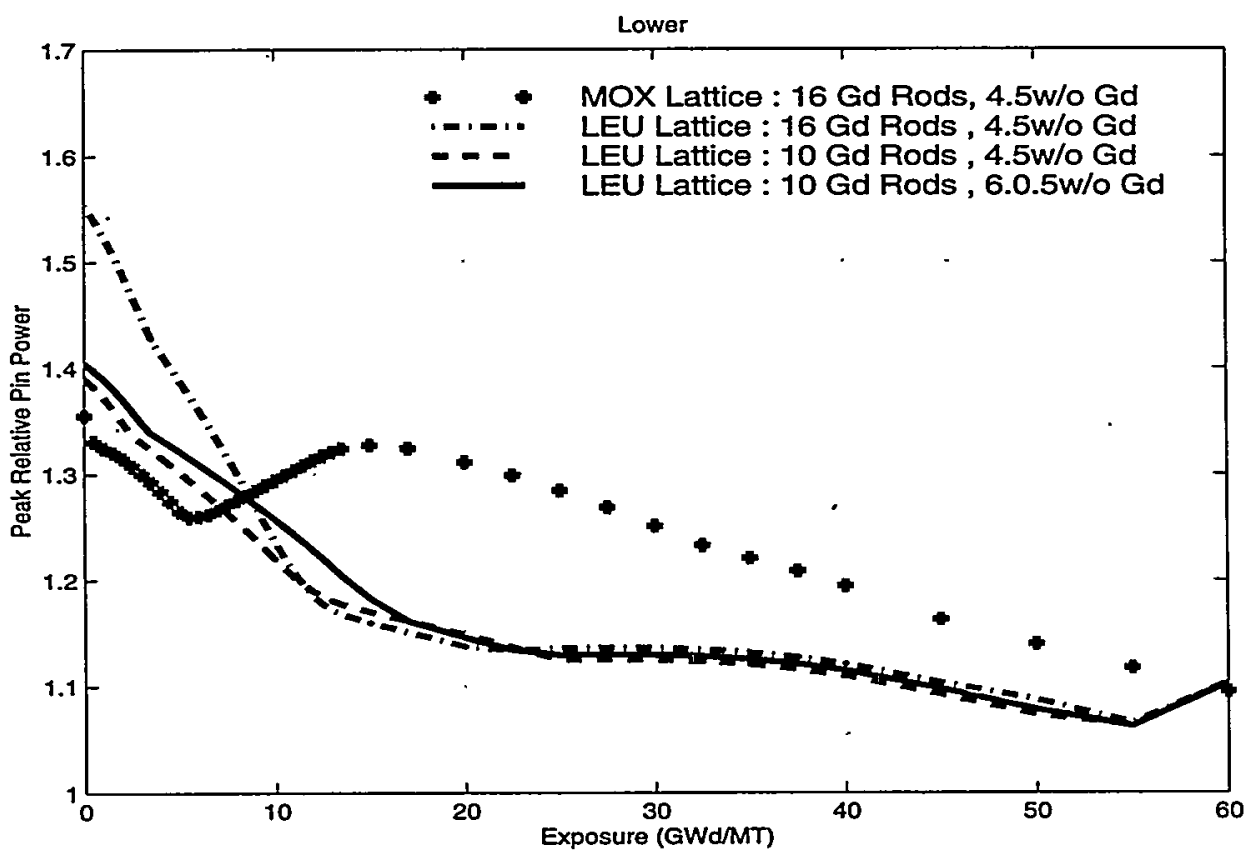

FIGURE 5.5. Lower Lattice LEU vs. MOX Lattice Peak Pin Power (Uncontrolled, HFP, $0 \%$ Void) 


\begin{tabular}{|c|c|c|c|c|c|c|c|c|}
\hline \multicolumn{9}{|c|}{ Upper } \\
\hline 2.20 & 3.00 & 3.60 & 4.20 & 4.20 & 4.20 & 3.60 & 3.00 & 2.20 \\
\hline & VR & 3.40 & $4.95^{*}$ & VR & $4.95^{*}$ & 3.40 & VR & 3.00 \\
\hline & & 4.60 & 4.60 & 4.95 & 4.60 & $4.95^{*}$ & 3.40 & 3.60 \\
\hline & & & 4.95 & $\mathrm{~W}$ & $W$ & 4.60 & $4.95 *$ & 4.20 \\
\hline & & & & W & $\mathrm{W}$ & 4.95 & VR & 4.20 \\
\hline & & & & & 4.95 & 4.60 & $4.95^{*}$ & 4.20 \\
\hline \multicolumn{4}{|c|}{ Numbers shown are U-235w/o } & & & 4.60 & 3.40 & 3.60 \\
\hline \multicolumn{4}{|c|}{ VR = Vanished Region } & & & & VR & 3.00 \\
\hline \multicolumn{4}{|c|}{$* \mathrm{Gd} 4.5 \mathrm{w} / \mathrm{o}$} & & & & & 2.20 \\
\hline
\end{tabular}

Middle

\begin{tabular}{|c|c|c|c|c|c|c|c|c|}
\hline 2.20 & 3.00 & 3.60 & 4.20 & 4.20 & 4.20 & 3.60 & 3.00 & 2.20 \\
\hline & 2.40 & $\cdot 3.40$ & $4.40^{*}$ & 4.40 & $4.40^{*}$ & 3.40 & 2.40 & 3.00 \\
\hline & & 4.60 & 4.60 & 4.40 & 4.60 & $4.40^{*}$ & 3.40 & 3.60 \\
\hline & & & 4.40 & W & W & 4.60 & $4.40^{*}$ & 4.20 \\
\hline . & & & & W & W & 4.40 & 4.40 & 4.20 \\
\hline & & & & & $\dot{4} .40$ & 4.60 & $4.40^{*}$ & 4.20 \\
\hline & & & & & & 4.60 & 3.40 & 3.60 \\
\hline & & & & & & & 2.40 & 3.00 \\
\hline \multicolumn{4}{|c|}{ * Gd 5.0w/o } & & & & & 2.20 \\
\hline
\end{tabular}

Lower

\begin{tabular}{|c|c|c|c|c|c|c|c|c|}
\hline 2.20 & 3.00 & 3.60 & 4.20 & 4.20 & 4.20 & 3.60 & 3.00 & 2.20 \\
\hline & 2.40 & 3.40 & $3.60 *$ & 4.40 & $3.60 *$ & 3.40 & 2.40 & 3.00 \\
\hline & & 4.60 & 4.60 & 3.60 & 4.40 & $3.60^{*}$ & 3.40 & 3.60 \\
\hline & & & 3.60 & W & W & .4 .60 & $3.60 *$ & 4.20 \\
\hline & & & & W & W & 3.60 & 4.40 & 4.20 \\
\hline & & & & & 3.60 & 4.60 & $3.60 *$ & 4.20 \\
\hline & & & & & & 4.60 & 3.40 & 3.60 \\
\hline & & & & & & & 2.40 & 3.00 \\
\hline \multicolumn{4}{|c|}{ * Gd 6.5w/o } & & & & & 2.20 \\
\hline
\end{tabular}

FIGURE 5.6. LEU Enrichment 1 Fuel Bundle Composition 
Since FORMOSA-B does not complete an enrichment search, the mean enrichment for the LEU feed assemblies which will meet the cycle energy production requirement and other constraints has to be predetermined. This can be done by providing FORMOSA-B with two LEU feed fuel bundle designs, one with excess reactivity and the other with deficient reactivity with regard to meeting the cycle energy production requirement and determining the appropriate mix to satisfy the cycle energy requirement. Two sets of LEU lattice cross sections were generated for this purpose, obtained by increasing or decreasing the pin-wise ${ }^{235} \mathrm{U}$ enrichment uniformly throughout the lattice from those used in the enrichment 1 lattices. Enrichment 2 (Figure 5.7) is generated by subtracting $0.15 \mathrm{w} / \mathrm{o}{ }^{235} \mathrm{U}$ from every LEU pin, while enrichment 3 (Figure 5.8) is generated by adding $0.15 \mathrm{w} / \mathrm{o}^{235} \mathrm{U}$ to every LEU pin. This results in a maximum $5.10 \mathrm{w} / \mathrm{o}$ ${ }^{235} \mathrm{U}$ pin enrichment, which slightly exceeds the fuel fabrication plant's current critical material license. Given the continuing need to increase the critical material license enrichment limit as required to support higher discharge burnups and the fact that the maximum enrichment occurs in a Gd rod, this slight violation should present no practical problem. Figure 5.9 present the lattice $\mathrm{k}_{\text {inf }}$ versus burnup for the axially lower MOX lattice and axially lower LEU enrichments 1,2 and 3 lattices, displaying the desired "bracketing" of the MOX lattice behavior.

Due to the limited time available, we did not utilize FORMOSA-B's capability to perform an LP search. Instead, the burned fuel bundles are kept at their original locations as defined in the equilibrium cycle, full MOX core LP (Figure 4.3). The mix of the two LEU assembly enrichments are varied such that the EOC core flow requirement is met. For each feed fuel variation, the CRP search capability in FORMOSA-B is utilized. Similar to the equilibrium cycle, full MOX core, the target $k_{\text {eff }}$ is 1.000 throughout the cycle depletion.

The CRP and LP for the resulting LP for the initial mixed MOX-LEU core are shown in Figure 5.10 and Figure 5.11, respectively. The core's key attributes are summarized in Table 5.2. MFLPD, MAPRAT, MFLCPR, maximum LHGR, minimum 
CPR and core flow values are comparable with the equilibrium cycle, full MOX core's values. Therefore, we conclude that a feasible initial mixed MOX-LEU core has been determined.

The resulting LP for the initial mixed MOX-LEU core is the starting point for the optimization of subsequent cycles as now explained. 
Upper

\begin{tabular}{|c|c|c|c|c|c|c|c|c|}
\hline 2.05 & 2.85 & 3.45 & 4.05 & 4.05 & 4.05 & 3.45 & 2.85 & 2.05 \\
\hline & VR & 3.25 & $4.80^{*}$ & VR & $4.80^{*}$ & 3.25 & VR & 2.85 \\
\hline & & 4.45 & 4.45 & 4.80 & 4.45 & $4.80 *$ & 3.25 & 3.45 \\
\hline & & & 4.80 & W & W & 4.45 & $4.80 *$ & 4.05 \\
\hline & & & & $\mathrm{W}$ & $\mathrm{W}$ & 4.80 & VR & 4.05 \\
\hline & & & & & 4.80 & 4.45 & $4.80^{*}$ & 4.05 \\
\hline \multicolumn{4}{|c|}{ Numbers shown are U-235w/o } & & & 4.45 & 3.25 & 3.45 \\
\hline \multicolumn{4}{|c|}{$\mathrm{VR}=$ Vanished Region } & & & & VR & 2.85 \\
\hline \multicolumn{4}{|c|}{ * Gd 4.5w/o } & & & & & 2.05 \\
\hline
\end{tabular}

Middle

\begin{tabular}{|c|c|c|c|c|c|c|c|c|}
\hline 2.05 & 2.85 & 3.45 & 4.05 & 4.05 & 4.05 & 3.45 & 2.85 & 2.05 \\
\hline & 2.25 & 3.25 & $4.25^{*}$ & 4.25 & $4.25^{*}$ & 3.25 & 2.25 & 2.85 \\
\hline & & 4.45 & 4.45 & 4.25 & 4.45 & $4.25^{*}$ & 3.25 & 3.45 \\
\hline & & & 4.25 & $\mathrm{~W}$ & $\mathrm{~W}$ & 4.45 & $4.25^{*}$ & 4.05 \\
\hline & & & & $\mathrm{W}$ & $\mathrm{W}$ & 4.25 & .4 .25 & 4.05 \\
\hline & & & & & 4.25 & 4.45 & $4.25 *$ & 4.05 \\
\hline & & & & & & 4.45 & 3.25 & 3.45 \\
\hline & & & & & & & 2.25 & 2.85 \\
\hline
\end{tabular}

Lower

\begin{tabular}{|c|c|c|c|c|c|c|c|c|}
\hline 2.05 & 2.85 & 3.45 & 4.05 & 4.05 & 4.05 & 3.45 & 2.85 & 2.05 \\
\hline & 2.25 & 3.25 & $3.45^{*}$ & 4.25 & $3.45^{*}$ & 3.25 & 2.25 & 2.85 \\
\hline & & 4.45 & 4.45 & 3.45 & 4.25 & $3.45^{*}$ & 3.25 & 3.45 \\
\hline & & & 3.45 & W & $\mathrm{W}$ & 4.45 & $3.45^{*}$ & 4.05 \\
\hline & & & & W & W & 3.45 & 4.25 & 4.05 \\
\hline & & & & & 3.45 & 4.45 & $3.45^{*}$ & 4.05 \\
\hline & & & & & & 4.45 & 3.25 & 3.45 \\
\hline & & & & & & & 2.25 & 2.85 \\
\hline \multicolumn{4}{|c|}{ * Gd 6.5w/o } & & & & & 2.05 \\
\hline
\end{tabular}

FIGURE 5.7. LEU Enrichment 2 Fuel Bundle Composition 


\section{Upper}

\begin{tabular}{|c|c|c|c|c|c|c|c|c|}
\hline 2.35 & 3.15 & 3.75 & 4.35 & 4.35 & 4.35 & 3.75 & 3.15 & 2.35 \\
\hline & VR & 3.55 & $5.10^{*}$ & VR & $5.10^{*}$ & 3.55 & VR & 3.15 \\
\hline & & 4.75 & 4.75 & 5.10 & 4.75 & $5.10^{*}$ & 3.55 & 3.75 \\
\hline & & & 5.10 & W & W & 4.75 & $5.10^{*}$ & 4.35 \\
\hline & & & & W & $\mathrm{W}$ & 5.10 & VR & 4.35 \\
\hline & & & & & 5.10 & 4.75 & $5.10^{*}$ & 4.35 \\
\hline \multicolumn{4}{|c|}{ Numbers shown are U-235w/o } & & & 4.75 & 3.55 & 3.75 \\
\hline \multicolumn{4}{|c|}{ VR = Vanished Region } & & & & VR & 3.15 \\
\hline \multicolumn{4}{|c|}{${ }^{*} \mathrm{Gd} 4.5 \mathrm{w} / \mathrm{o}$} & & & & & 2.35 \\
\hline
\end{tabular}

Middle

\begin{tabular}{|c|c|c|c|c|c|c|c|c|}
\hline 2.35 & 3.15 & 3.75 & 4.35 & 4.35 & 4.35 & 3.75 & 3.15 & 2.35 \\
\hline & 2.55 & 3.55 & $4.55^{*}$ & 4.55 & $4.55^{*}$ & 3.55 & 2.55 & 3.15 \\
\hline & & 4.75 & 4.75 & 4.55 & 4.75 & $4.55^{*}$ & 3.55 & 3.75 \\
\hline & & & 4.55 & $\mathrm{~W}$ & $\mathrm{~W}$ & 4.75 & $4.55^{*}$ & 4.35 \\
\hline & $\cdot$. & & & $\mathrm{W}$ & $\mathrm{W}$ & 4.55 & 4.55 & 4.35 \\
\hline & & & & & 4.55 & 4.75 & $4.55 *$ & 4.35 \\
\hline & & & & & & 4.75 & 3.55 & 3.75 \\
\hline & & & & & & & 2.55 & 3.15 \\
\hline
\end{tabular}

Lower

\begin{tabular}{|c|c|c|c|c|c|c|c|c|}
\hline 2.35 & 3.15 & 3.75 & 4.35 & 4.35 & 4.35 & 3.75 & 3.15 & 2.35 \\
\hline & 2.55 & 3.55 & $3.75^{*}$ & 4.55 & $3.75^{*}$ & 3.55 & 2.55 & 3.15 \\
\hline & $\cdot$ & 4.75 & 4.75 & 3.75 & 4.55 & $3.75^{*}$ & 3.55 & 3.75 \\
\hline & & & 3.75 & $\mathrm{~W}$ & $\mathrm{~W}$ & 4.75 & $3.75^{*}$ & 4.35 \\
\hline & & & & $\mathrm{W}$ & $\mathrm{W}$ & 3.75 & 4.55 & 4.35 \\
\hline & & & & & 3.75 & 4.75 & $3.75 *$ & 4.35 \\
\hline & & & & & & 4.75 & 3.55 & 3.75 \\
\hline & & & & & & & 2.55 & 3.15 \\
\hline
\end{tabular}

FIGURE 5.8. LEU Enrichment 3 Fuel Bundle Composition 


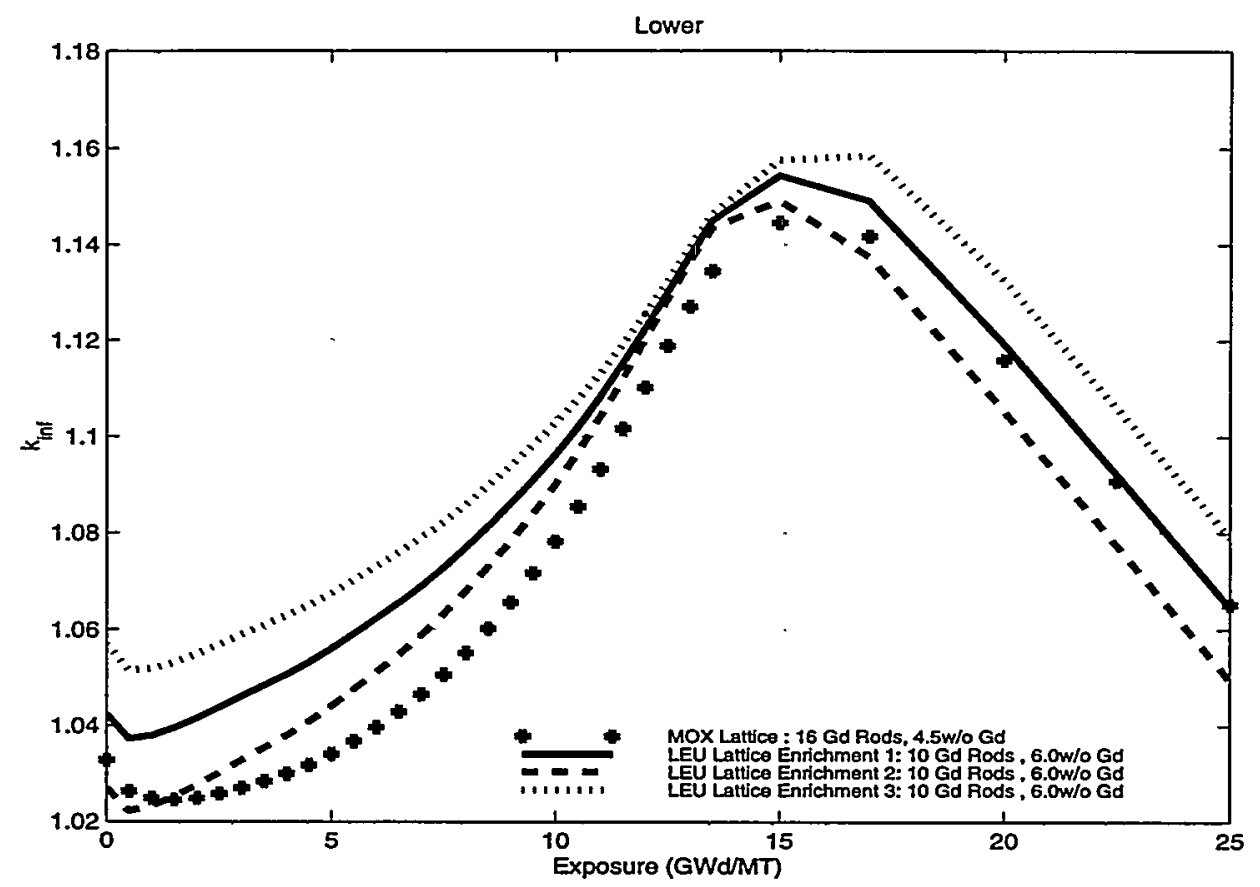

FIGURE 5.9. Lower Lattice $k_{\text {inf }}$ for Different LEU Enrichments (Uncontrolled, HFP, $0 \%$ Void)

TABLE 5.2: Initial Mixed MOX-LEU Core's Key Attributes

\begin{tabular}{|c|cccccccc|}
\hline $\begin{array}{c}\text { Cycle } \\
\text { Exposure } \\
\text { (GWd/ST) }\end{array}$ & $\begin{array}{c}\text { \% Rated } \\
\text { Core } \\
\text { Flow }\end{array}$ & MFLPD & MAPRAT & MFLCPR & $\begin{array}{c}\text { LHGR } \\
\text { kw/f. }\end{array}$ & MCPR & $\begin{array}{c}\text { Mo0\% Core } \\
\text { Flow) }\end{array}$ \\
\hline \hline 0 & .84 .097 & 0.928 & 0.825 & 0.908 & 12.029 & 1.531 & 1.0218 \\
1.5 & 84.063 & $\mathbf{0 . 9 4 4}$ & 0.852 & $\mathbf{0 . 9 4 2}$ & 12.231 & 1.476 & 1.0283 \\
3.0 & 85.042 & 0.931 & 0.836 & 0.932 & 12.061 & 1.492 & 1.0271 \\
4.5 & 85.551 & 0.847 & 0.822 & 0.913 & 10.973 & 1.522 & 1.0263 \\
6.0 & 91.116 & 0.829 & 0.803 & 0.933 & 10.748 & 1.490 & 1.0265 \\
7.5 & 86.545 & 0.858 & $\mathbf{0 . 8 9 5}$ & 0.939 & 11.123 & 1.480 & 1.0268 \\
9.0 & 99.769 & 0.807 & 0.821 & 0.930 & 10.462 & 1.494 & 1.0234 \\
10.5 & 87.864 & 0.804 & 0.780 & 0.920 & 10.421 & 1.511 & 1.0152 \\
11.5 & 94.142 & 0.779 & 0.781 & 0.888 & 10.100 & 1.565 & 1.0082 \\
12.35 & $\mathbf{1 0 5 . 4 2 2}$ & 0.726 & 0.812 & 0.863 & 9.403 & 1.611 & 1.0016 \\
\hline
\end{tabular}



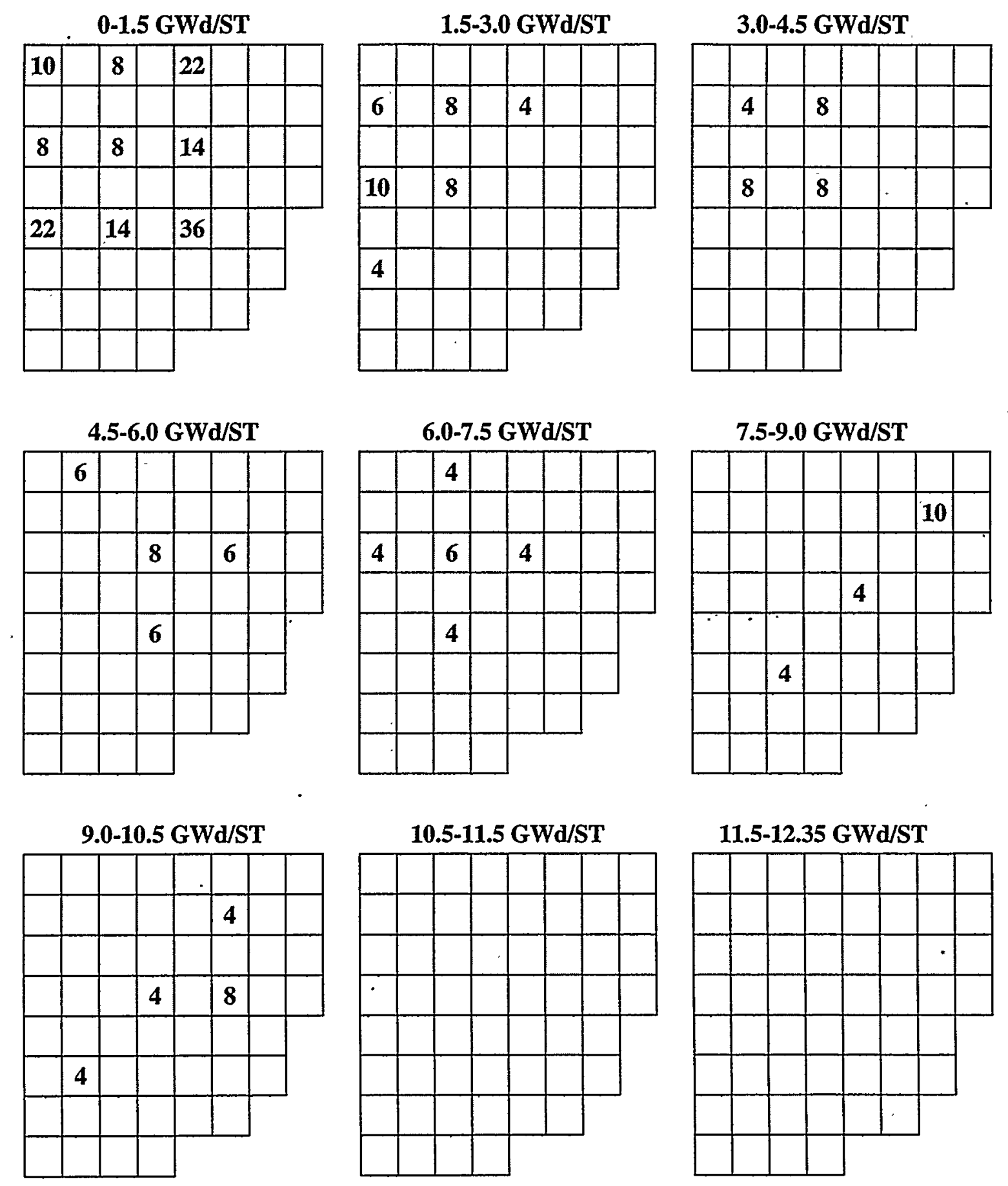

FIGURE 5.10. Initial Mixed Core Control Rod Patterns 
First Index

\begin{tabular}{|c|c|c|c|c|c|c|c|c|c|c|c|c|c|c|c|c|}
\hline & 16 & 15 & 14 & 13 & 12 & 11 & 10 & 9 & 8 & 7 & 6 & 5 & 4 & 3 & 2 & 1 \\
\hline 16 & 2 & 1 & 1 & 1 & 1 & 1 & 1 & 2 & $\mathrm{~F} 2$ & 2 & F3 & 2 & F3 & 1 & F3 & 2 \\
\hline 15 & 1 & $\mathrm{~F} 2$ & 2 & $\mathrm{~F} 2$ & 2 & $\mathrm{~F} 2$ & 2 & $\mathrm{~F} 2$ & 2 & F2 & 2 & $\mathrm{~F} 2$ & 2 & F3 & 1 & 3 \\
\hline 14 & 1 & 2 & 1 & 1 & 1 & 1 & 1 & 1 & $\mathrm{~F} 2$ & 1 & F2 & 1 & F3 & 2 & 2 & \\
\hline 13 & 1 & F2 & 1 & F2 & 2 & $\mathrm{~F} 2$ & 2 & F2 & 2 & F2 & 2 & F3 & 2 & 1 & 2 & \\
\hline 12 & 1 & 2 & 1 & 2 & 1 & 1 & 1 & 1 & $\mathrm{~F} 2$ & 1 & F2 & 1 & F3 & 1 & 2 & \\
\hline 11 & 1 & $\mathrm{~F} 2$ & 1 & $\mathrm{~F} 2$ & 1 & F2 & 2 & $\mathrm{~F} 2$ & 2 & F2 & 2 & F3 & 2 & 1 & 2 & \\
\hline 10 & 1 & 2 & 1 & 2 & 1 & 2 & F3 & 1 & F3 & 1 & F3 & 1 & F3 & 1 & 2 & \\
\hline 9 & 2 & F2 & 1 & $\mathrm{~F} 2$ & 1 & F2 & 1 & F3 & 2 & F3 & 2 & F3 & 1 & 2 & & \\
\hline 8 & F2 & 2 & F2 & 2 & $\mathrm{~F} 2$ & 2 & F3 & 2 & F3 & 1 & F3 & 1 & 2 & & & \\
\hline 7 & 2 & F2 & 1 & F2 & 1 & F2 & 1 & F3 & 1 & F3 & 2 & 1 & 2 & & & \\
\hline 6 & F3 & 2 & F2 & 2 & $\mathrm{~F} 2$ & 2 & F3 & 2 & F3 & 2 & F3 & .1 & 2 & & & \\
\hline 5 & 2 & F2 & 1 & F3 & 1 & F3 & 1 & F3 & 1 & 1 & 1 & 2 & & & & \\
\hline 4 & F3 & 2 & F3 & 2 & F3 & 2 & F3 & 1 & 2 & 2 & 2 & & & & & \\
\hline 3 & 1 & F3 & 2 & 1 & 1 & 1 & 1 & 2 & & & & & & & & \\
\hline 2 & F3 & 1 & 2 & 2 & 2 & 2 & 2 & & & & & & & & & \\
\hline 1 & 2 & 3 & & & & & & & & & & & & & & \\
\hline
\end{tabular}

The numbers represent the number of previous cycles the bundle has been in the core. The symbol $F$ denotes fresh fuels.

$$
\begin{aligned}
& \text { F2 }=\text { LEU Enrichment } 2 \text { (Figure 5.7) } \\
& \text { F3 }=\text { LEU Enrichment } 3 \text { (Figure 5.8) }
\end{aligned}
$$

FIGURE 5.11. Initial Mixed MOX-LEU Core Loading Pattern 


\section{Subsequent Mixed MOX-LEU Cores}

Similar to the mixed MOX-LEU core studies completed for PWRs [1 and 2], starting with the initial mixed MOX-LEU core defined in Section 5, two scenarios (see Figure 6.1) are introduced in regard to availability of MOX feed assemblies in the subsequent cycle: (1) assume that MOX feed batches are available and (2) assume that MOX feed batches continue to be unavailable. MOX lattice designs are limited to MOX designs used in the GE BWR-6 equilibrium cycle, full MOX core and LEU lattice designs are limited to the two designs described in Section 5. This was done to determine if acceptable LPs could be determined without the need to develop new and more complex lattice designs.

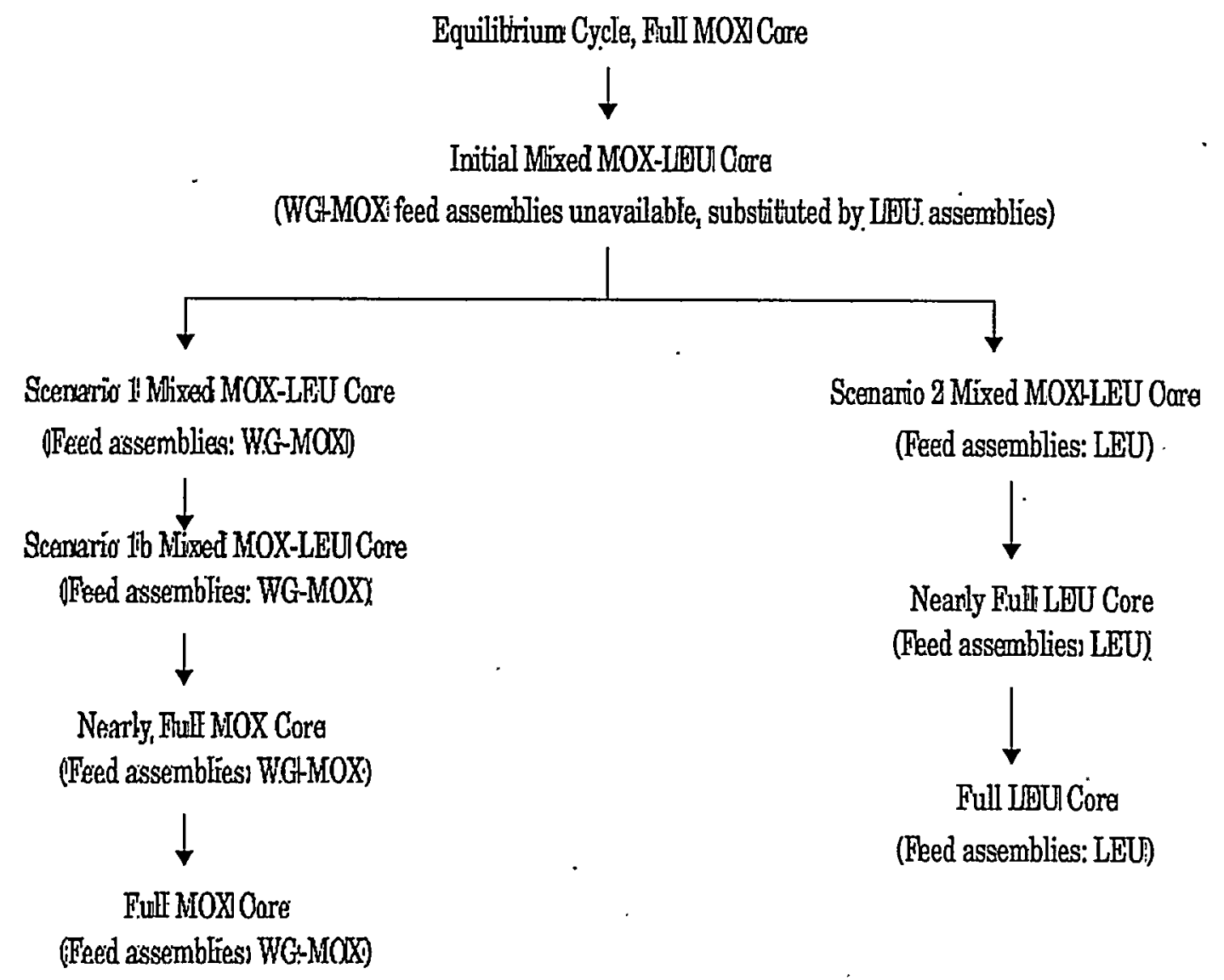

FIGURE 6.1. Mixed MOX-LEU Core Disruption Scenarios 


\subsection{Scenario 1: MOX Feed Assemblies Available for Subsequent Cycles}

For scenario 1 cores, the LP is created by using the shuffle instructions shown in Figure 4.3. The same MOX feed fuel assembly design as employed in the equilibrium cycle, full MOX core is again used. The CRP is obtained utilizing FORMOSA-B's CRP search capability with core flow adjusted to achieve the target $k_{\text {eff }}$. As before, the target $\mathrm{k}_{\text {eff }}$ is kept at 1.000 throughout the cycle depletion. Thermal margin and core flow values summarized in Table 6.1 show that a feasible LP/CRP has been obtained. This core will be referred to as the scenario 1 mixed MOX-LEU core whose LP and CRP are shown in Figure 6.2 and Figure 6.3, respectively.

TABLE 6.1: Scenario 1 Mixed MOX-LEU Core's Key Attributes

\begin{tabular}{|c|cccccccc|}
\hline $\begin{array}{c}\text { Cycle } \\
\text { Exposure } \\
\text { (GWd/ST) }\end{array}$ & $\begin{array}{c}\text { \% Rated } \\
\text { Core } \\
\text { Flow }\end{array}$ & MFLPD & MAPRAT & MFLCPR & $\begin{array}{c}\text { MHGR. } \\
\text { kw/ft. }\end{array}$ & MCPR & $\begin{array}{c}\text { 100\% Core } \\
\text { Flow) }\end{array}$ \\
\hline \hline 0 & 83.402 & $\mathbf{0 . 9 4 0}$ & 0.864 & 0.926 & 12.183 & 1.500 & 1.0191 \\
1.5 & 83.113 & 0.888 & 0.830 & 0.907 & 11.506 & 1.533 & 1.0208 \\
3.0 & 85.040 & 0.822 & 0.794 & 0.911 & 10.654 & 1.525 & 1.0166 \\
4.5 & 84.736 & 0.902 & $\mathbf{0 . 9 1 1}$ & 0.933 & 11.692 & 1.490 & 1.0135 \\
6.0 & 82.999 & 0.866 & 0.853 & 0.910 & 11.218 & 1.527 & 1.0121 \\
7.5 & 93.177 & 0.910 & 0.875 & 0.938 & 11.790 & 1.481 & 1.0118 \\
9.0 & 84.096 & 0.939 & 0.902 & $\mathbf{0 . 9 4 2}$ & 12.173 & 1.476 & 1.0106 \\
10.5 & 87.805 & 0.905 & 0.796 & 0.894 & 11.734 & 1.554 & 1.0069 \\
11.5 & 95.541 & 0.834 & 0.819 & 0.880 & 10.812 & 1.579 & 1.0027 \\
12.35 & $\mathbf{1 0 4 . 6 6 5}$ & 0.834 & 0.853 & 0.878 & 10.803 & 1.583 & 0.9981 \\
\hline
\end{tabular}


First Index

\begin{tabular}{|c|c|c|c|c|c|c|c|c|c|c|c|c|c|c|c|c|}
\hline & 16 & 15 & 14 & 13 & 12 & 11 & 10 & 9 & 8 & 7 & 6 & 5 & 4 & 3 & 2 & 1 \\
\hline 16 & 2 & 1 & 1 & 1 & 1 & 1 & 1 & 2 & F & 2 & $F$ & 2 & $F$ & 1 & $\mathrm{~F}$ & 2 \\
\hline 15 & 1 & $\mathrm{~F}$ & 2 & $\mathrm{~F}$ & 2 & $\mathrm{~F}$ & 2 & $F$ & 2 & $F$ & 2 & $F$ & 2 & $\mathrm{~F}$ & 1 & 3 \\
\hline 14 & 1 & 2 & 1 & 1 & 1 & 1 & 1 & 1 & $\mathrm{~F}$ & 1 & $\mathrm{~F}$ & 1 & $\mathrm{~F}$ & 2 & 2 & \\
\hline 13 & 1 & F & 1 & $\mathrm{~F}$ & 2 & $F$ & 2 & $F$ & 2 & $F$ & 2 & $F$ & 2 & 1 & 2 & \\
\hline 12 & 1 & 2 & 1 & 2 & 1 & 1 & 1 & 1 & $\mathrm{~F}$ & 1 & $\mathrm{~F}$ & 1 & $\mathrm{~F}$ & 1 & 2 & \\
\hline 11 & 1 & $F$ & 1 & $\mathrm{~F}$ & 1 & $\mathrm{~F}$ & 2 & $F$ & 2 & $\mathrm{~F}$ & 2 & $\mathrm{~F}$ & 2 & 1 & 2 & \\
\hline 10 & 1 & 2 & 1 & 2 & 1 & 2 & $\mathrm{~F}$ & 1 & $\mathrm{~F}$ & 1 & $\mathrm{~F}$ & 1 & $F$ & 1 & 2 & \\
\hline 9 & 2 & $\mathrm{~F}$ & 1 & $\mathrm{~F}$ & 1 & $\mathrm{~F}$ & 1 & $\mathrm{~F}$ & 2 & $\mathrm{~F}$ & 2 & $\mathrm{~F}$ & 1 & 2 & & \\
\hline 8 & $\mathrm{~F}$ & 2 & $F$ & 2 & $\mathrm{~F}$ & 2 & $\mathrm{~F}$ & 2 & $\mathrm{~F}$ & 1 & $\mathrm{~F}$ & 1 & 2 & & & \\
\hline 7 & 2 & $F$ & 1 & $F$ & 1 & $\mathrm{~F}$ & 1 & $F$ & 1 & $\mathrm{~F}$ & 2 & 1 & 2 & & & \\
\hline 6 & $\mathrm{~F}$ & 2 & $\mathrm{~F}$ & 2 & $\mathrm{~F}$ & 2 & $\mathrm{~F}$ & 2 & $\mathrm{~F}$ & 2 & $F$ & 1 & 2 & . & & \\
\hline 5 & 2 & $\mathrm{~F}$ & 1 & $\mathrm{~F}$ & 1 & $\mathrm{~F}$ & 1 & $\mathrm{~F}$ & 1 & 1 & 1 & 2 & & & & \\
\hline 4 & $\mathrm{~F}$ & 2 & $\mathrm{~F}$ & 2 & $\mathrm{~F}$ & 2 & $\mathrm{~F}$ & 1 & 2 & 2 & 2 & & & & & \\
\hline 3 & 1 & $F$ & 2 & 1 & 1 & 1 & 1 & 2 & & & & & & & & \\
\hline 2 & $\mathrm{~F}$ & 1 & 2 & 2 & 2 & 2 & 2 & & & & & & & & & \\
\hline 1 & 2 & 3 & & & & & & & & & & & & & & \\
\hline
\end{tabular}

The numbers represent the number of previous cycles the bundle has been in the core.

$\mathrm{F}=$ MOX Fresh Assemblies

FIGURE 6.2. Scenario 1 Mixed MOX-LEU Core Loading Pattern 
0-1.5 GWd/ST

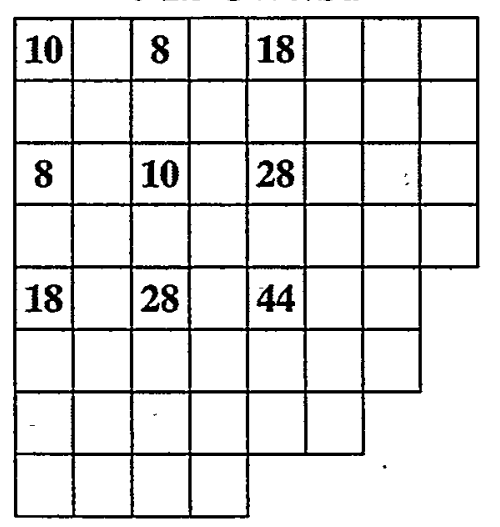

4.5-6.0 GWd/ST

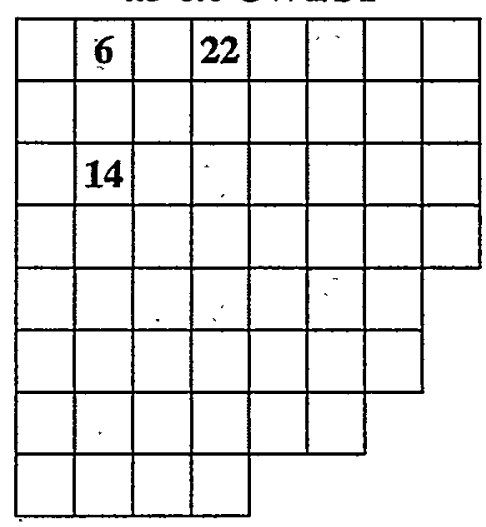

9.0-10.5 GWd/ST

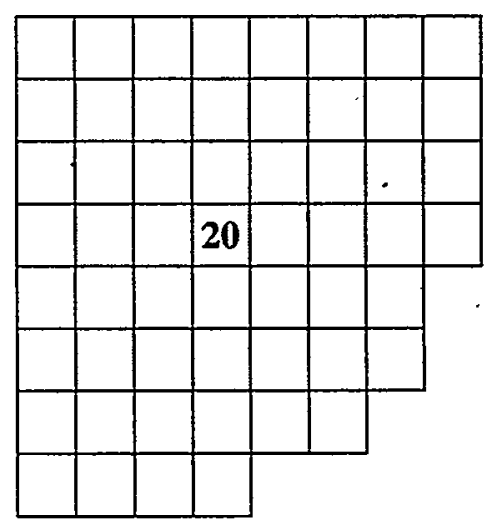

1.5-3.0. GWd/ST

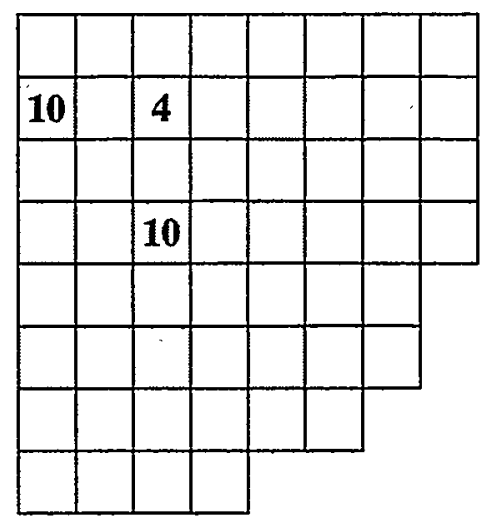

6.0-7.5 GWd/ST

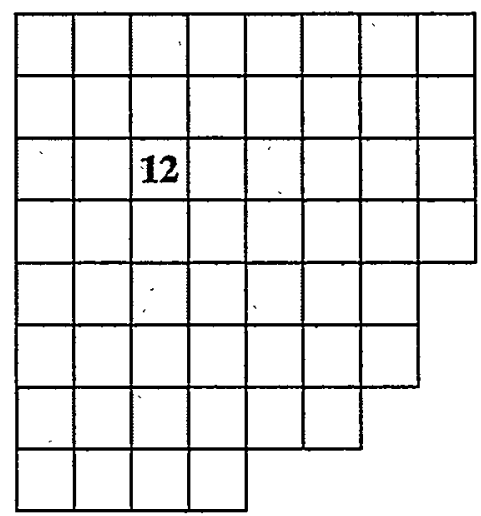

10.5-11.5 GWd/ST.

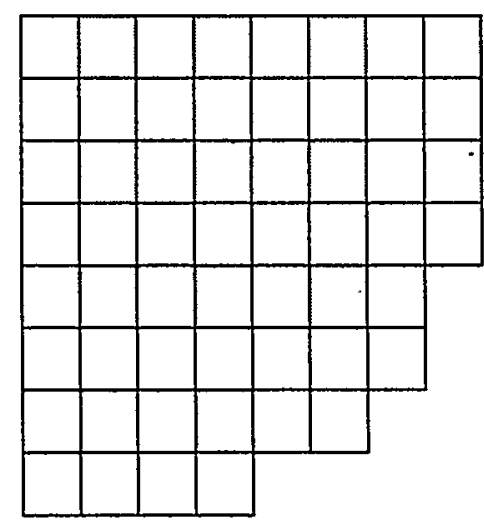

3.0-4.5 GWd/ST

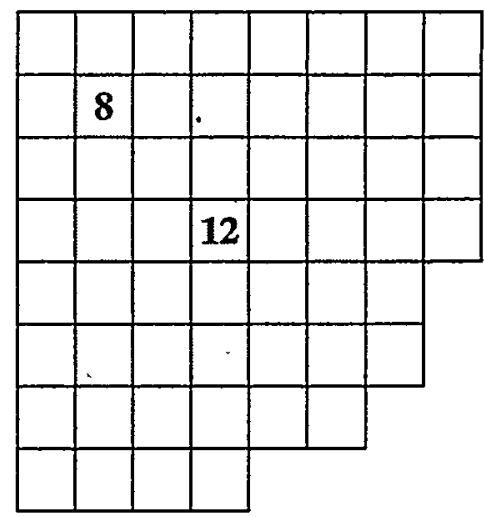

7.5-9.0 GWd/ST

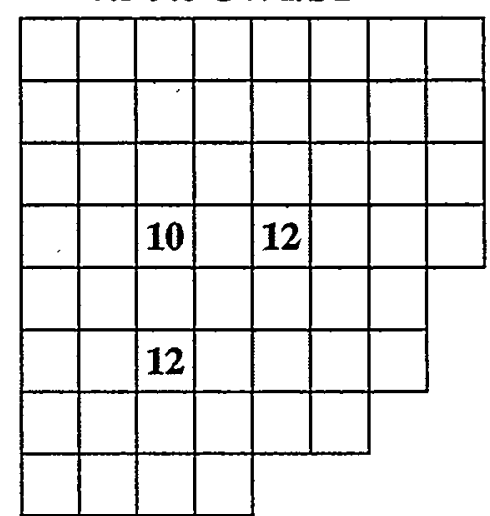

11.5-12.35 GWd/ST

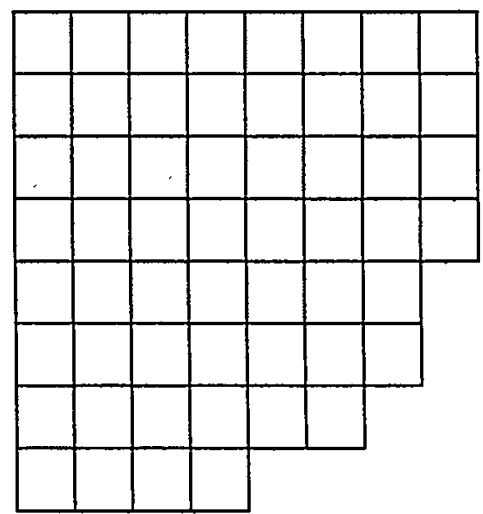

FIGURE 6.3. Scenario 1 Mixed MOX-LEU Core Control Rod Patterns 


\subsection{Transition Back to Full MOX Core}

\subsubsection{Scenario 1b Mixed MOX-LEU Core}

The scenario $1 \mathrm{~b}$ mixed MOX-LEU core is obtained by loading MOX feed assemblies in the subsequent cycle of the scenario 1 mixed MOX-LEU core. In the scenario $1 \mathrm{~b}$ mixed MOX-LEU core, there are 8 thrice-burned MOX assemblies, 264 twice-burned LEU assemblies, 264 once-burned MOX assemblies, and 264 fresh MOX assemblies. The CRP for this core is shown in Figure 6.4. A summary of thermal margin and core flow values are shown in Table 6.2. Acceptable thermal margin values are observed. It is noted that the EOC core flow is higher than the specified limit of $105 \%$. Recall that the LP for this core is obtained using the shuffle instructions employed by the equilibrium cycle, full MOX core without any further LP optimization. Currently, employing the FORMOSA-B's LP search capability requires a substantial amount of computational time, hence the desire to avoid the need to complete LP optimization.

TABLE 6.2: Scenario 1b Mixed MOX-LEU Core's Key Attributes

\begin{tabular}{|c|cccccccc|}
\hline $\begin{array}{c}\text { Cycle } \\
\text { Exposure } \\
\text { (GWd/ST) }\end{array}$ & $\begin{array}{c}\text { \% Rated } \\
\text { Core } \\
\text { Flow }\end{array}$ & MFLPD & MAPRAT & MFLCPR & $\begin{array}{c}\text { LHGR } \\
\text { kw/ft. }\end{array}$ & MCPR & $\begin{array}{c}\text { 100\% Core } \\
\text { Flow) }\end{array}$ \\
\hline \hline 0 & 85.300 & 0.935 & 0.863 & 0.898 & 12.118 & 1.548 & 1.0185 \\
1.5 & 85.555 & 0.875 & 0.812 & 0.892 & 11.343 & 1.558 & 1.0205 \\
3.0 & 89.292 & 0.895 & 0.879 & 0.936 & 11.597 & 1.485 & 1.0166 \\
4.5 & 85.981 & 0.921 & $\mathbf{0 . 8 8 4}$ & 0.919 & 11.941 & 1.512 & 1.0139 \\
6.0 & 80.698 & $\mathbf{0 . 9 5 0}$ & 0.883 & $\mathbf{0 . 9 4 9}$ & 12.305 & 1.465 & 1.0127 \\
7.5 & 89.626 & 0.928 & 0.871 & 0.952 & 12.029 & 1.460 & 1.0127 \\
9.0 & 85.484 & 0.864 & 0.854 & 0.951 & 11.192 & 1.462 & 1.0114 \\
10.5 & 89.108 & 0.889 & 0.807 & 0.897 & 11.518 & 1.550 & 1.0068 \\
11.5 & 97.499 & 0.860 & 0.811 & 0.870 & 11.146 & 1.598 & 1.0017 \\
12.35 & 108.259 & 0.828 & 0.858 & 0.865 & 10.728 & 1.607 & 0.9966 \\
\hline
\end{tabular}


0-1.5 GWd/ST

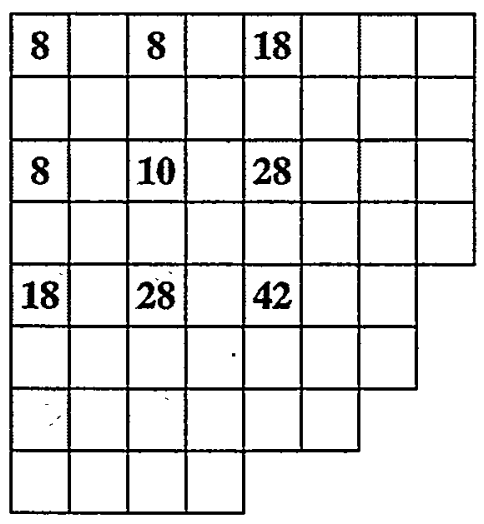

4.5-6.0 GWd/ST

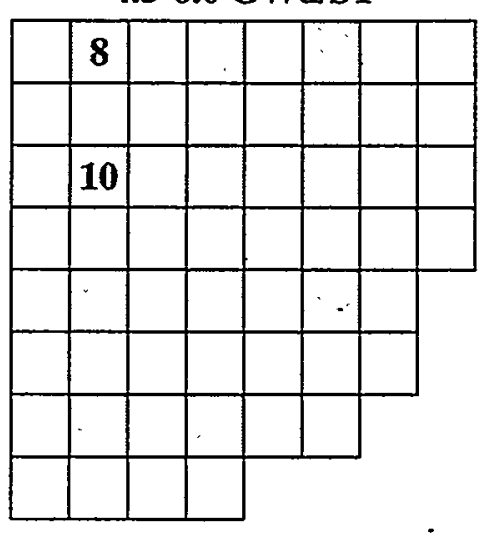

9.0-10.5 GWd/ST

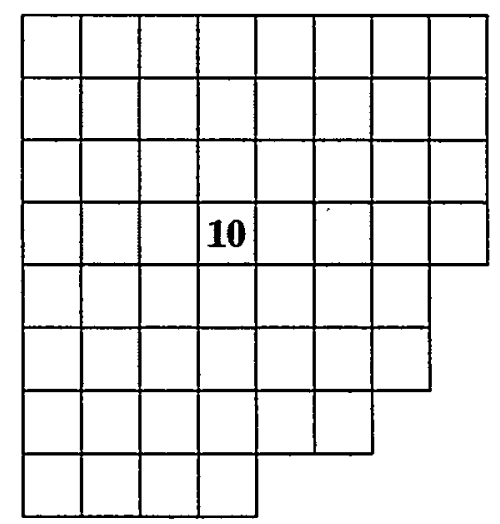

1.5-3.0 GWd/ST

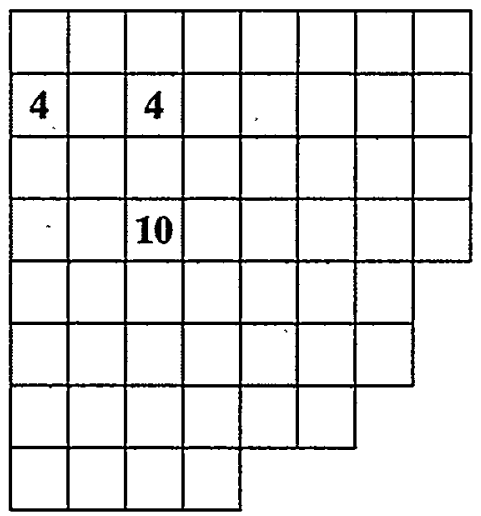

6.0-7.5 GWd/ST

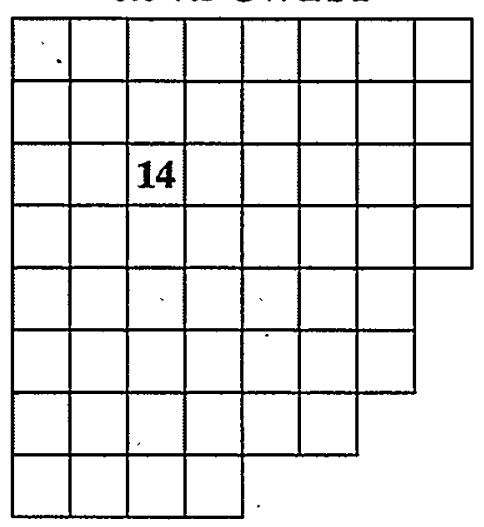

10.5-11.5 GWd/ST

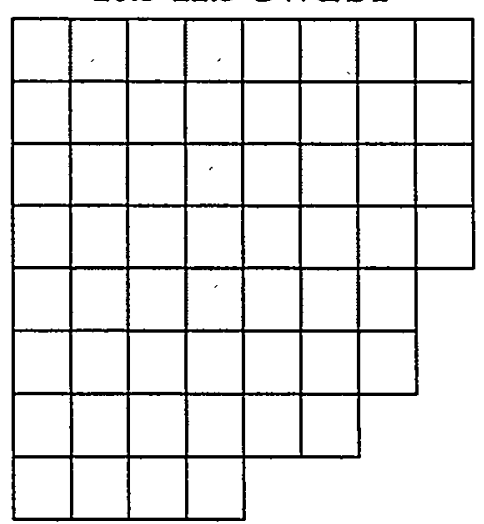

3.0-4.5 GWd/ST

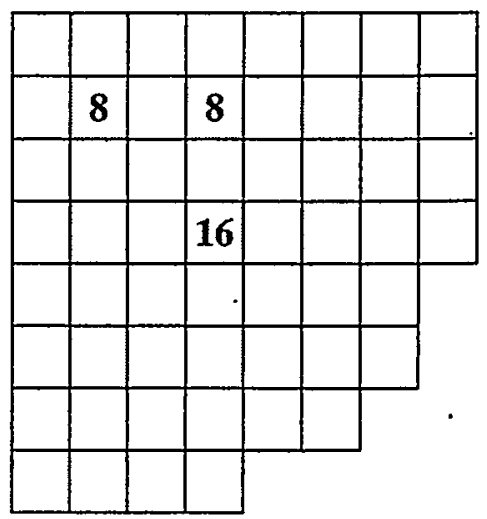

7.5-9.0 GWd/ST

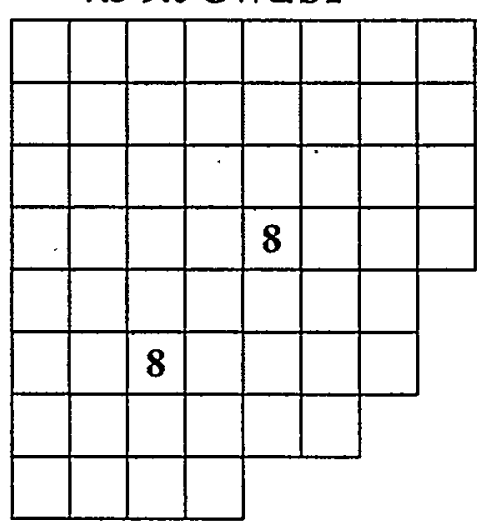

11.5-12.35 GWd/ST

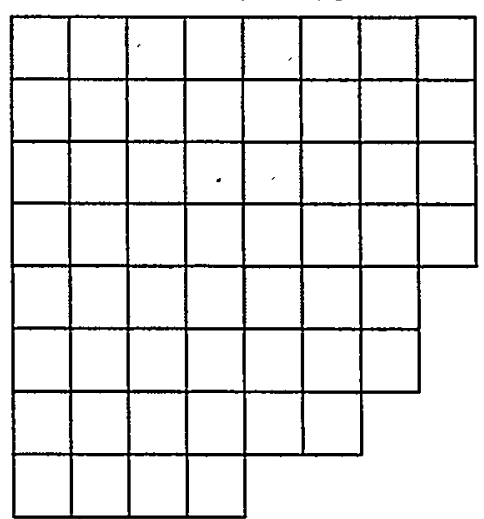

FIGURE 6.4. Scenario 1b Mixed MOX-LEU Core Control Rod Patterns 
Performing LP/CRP search on the scenario $1 \mathrm{~b}$ mixed MOX-LEU core removes the EOC core flow violation and also reduces the.maximum thermal margin values, as shown in Table 6.3. The objective function employed during the Optimization by Simulated Annealing (OSA) cooling cycle is cycle length maximization. The OSA search type is a local search with 2,000 histories and a $k_{\text {inf }}$ range of 0.18 . The $k_{\text {inf }}$ range allows the exchange between once- and twice-burned assemblies. The locations of fresh MOX assemblies are preserved during OSA. A CRP search is performed every 200 histories. The optimized LP and CRP are shown in Figure 6.5 and Figure 6.6, respectively.

TABLE 6.3: Scenario 1b (Optimized) Mixed MOX-LEU Core's Key Attributes

\begin{tabular}{|c|cccccccc|}
\hline $\begin{array}{c}\text { Cycle } \\
\text { Exposure } \\
\text { (GWd/ST) }\end{array}$ & $\begin{array}{c}\text { \% Rated } \\
\text { Core } \\
\text { Flow }\end{array}$ & MFLPD & MAPRAT & MFLCPR & $\begin{array}{c}\text { LHGR/ft. } \\
\text { kw/ }\end{array}$ & MCPR & $\begin{array}{c}\text { 100\% Core } \\
\text { Flow) }\end{array}$ \\
\hline \hline 0 & 85.604 & 0.863 & $\mathbf{0 . 8 5 2}$ & 0.901 & 11.188 & 1.542 & 1.0171 \\
1.5 & 86.077 & $\mathbf{0 . 9 2 7}$ & 0.808 & 0.852 & 12.013 & 1.631 & 1.0193 \\
3.0 & 86.224 & 0.933 & 0.844 & 0.884 & 12.097 & 1.573 & 1.0159 \\
4.5 & 85.547 & 0.874 & 0.795 & 0.898 & 11.330 & 1.547 & 1.0134 \\
6.0 & 85.078 & 0.920 & 0.803 & 0.917 & 11.919 & 1.516 & 1.0123 \\
7.5 & 87.627 & 0.867 & 0.788 & 0.915 & 11.232 & 1.520 & 1.0125 \\
9.0 & 96.032 & 0.901 & 0.841 & 0.908. & 11.677 & 1.531 & 1.0118 \\
10.5 & 89.059 & 0.885 & 0.797 & 0.916 & 11.465 & 1.518 & 1.0083 \\
11.5 & 94.142 & 0.840 & 0.816 & 0.907 & 10.884 & 1.532 & 1.0041 \\
12.35 & $\mathbf{1 0 3 . 5 3 9}$ & 0.863 & 0.852 & 0.901 & 11.188 & 1.542 & 0.9994 \\
\hline
\end{tabular}


First Index

\begin{tabular}{|c|c|c|c|c|c|c|c|c|c|c|c|c|c|c|c|c|}
\hline & 16 & 15 & 14 & 13 & 12 & 11 & 10 & 9 . & 8 & 7 & 6 & 5 & 4 & 3 & 2 & 1 \\
\hline 16 & 2 & 1 & 1 & 1 & 1 & 2 & 1 & 2 & $F$ & 2 & $\mathrm{~F}$ & 2 & $\mathrm{~F}$ & 1 & $\mathrm{~F}$ & 2 \\
\hline 15 & 2 & F & 1 & $\mathrm{~F}$ & 2 & $\mathrm{~F}$ & 2 & $F$ & 2 & $F$ & 1 & $F$ & 1 & $\mathrm{~F}$ & 1 & 2 \\
\hline 14 & 1 & 2 & 1 & 1 & 1 & 2 & 1 & 1 & $\mathrm{~F}$ & 1 & $\mathrm{~F}$ & 2 & F & 1 & 2 & \\
\hline 13 & 1 & $\mathrm{~F}$ & 1 & $\mathrm{~F}$ & 2 & $F$ & 2 & $\mathrm{~F}$ & 2 & $\mathrm{~F}$ & 2 & $\mathrm{~F}$ & 1 & 1 & 2 & \\
\hline 12 & 1 & 2 & 1 & 2 & 1 & 2 & 1 & 2 & $\mathrm{~F}$ & 2 & $\mathrm{~F}$ & 1 & $F$ & 2 & 2 & \\
\hline 11 & 1 & $\mathrm{~F}$ & 2 & $\mathrm{~F}$ & 1 & $\mathrm{~F}$ & 2 & $\mathrm{~F}$ & 2 & $\mathrm{~F}$ & 1 & $\mathrm{~F}$ & 1 & 1 & 2 & \\
\hline 10 & 1 & 2 & 1 & 1 & 1 & 2 & $\mathrm{~F}$ & 1 & $\mathrm{~F}$ & 1 & $\mathrm{~F}$ & 1 & $\mathrm{~F}$ & 2 & 2 & \\
\hline 9 & 2 & F & 1 & $F$ & 1 & F & 1 & $\mathrm{~F}$ & 1 & $\mathrm{~F}$ & 2 & $\mathrm{~F}$ & 2 & 1 & & \\
\hline 8 & $F$ & 2 & $\mathrm{~F}$ & 1 & $\mathrm{~F}$ & 2 & $\mathrm{~F}$ & 1 & $\mathrm{~F}$ & 2 & $\mathrm{~F}$ & 1 & 2 & & & \\
\hline 7 & 2 & $\mathrm{~F}$ & 1 & $\mathrm{~F}$ & 1 & $F$ & 2 & $\mathrm{~F}$ & 1 & $\mathrm{~F}$ & 2 & 1 & 3 & & & \\
\hline 6 & $F$ & 2 & $\bar{F}$ & 1 & $\mathrm{~F}$ & 1 & $\mathrm{~F}$ & 2 & $\mathrm{~F}$ & 1 & $\mathrm{~F}$ & 1 & 2 & & & \\
\hline 5 & 1 & $F$ & 1 & $F$ & 2 & $\mathrm{~F}$ & 1 & $\mathrm{~F}$ & 1 & 2 & 1 & 3 & & & & \\
\hline 4 & $\mathrm{~F}$ & 2 & $F$ & 1 & $\mathrm{~F}$ & 1 & $\mathrm{~F}$ & 1. & 2 & 2 & 2 & & & & & \\
\hline 3 & 1 & $\mathrm{~F}$ & 2 & 1 & 2 & 1 & 2 & 2 & & & & & & & & \\
\hline 2 & $\mathrm{~F}$ & 1 & 2 & 2 & 2 & 2 & 2 & & & & & & & & & \\
\hline 1 & 2 & 2 & & & & & & & & & & & & & & \\
\hline
\end{tabular}

The numbers represent the number of previous cycles the bundle has been in the core.

$\mathrm{F}=$ MOX Fresh Assemblies

FIGURE 6.5. Scenario 1b (Optimized) Mixed MOX-LEU Core Loading Pattern 

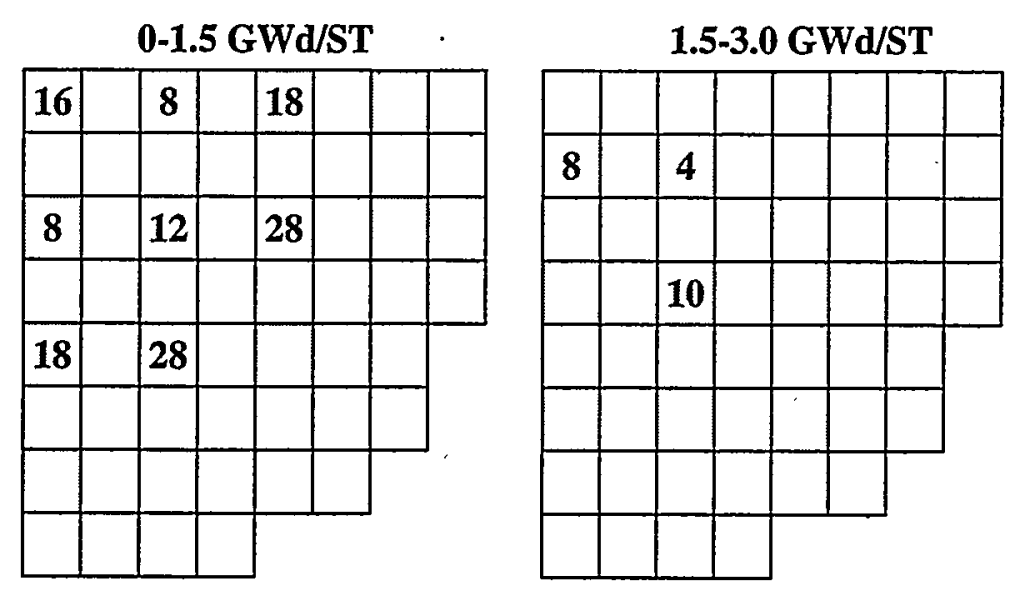

3.0-4.5 GWd/ST

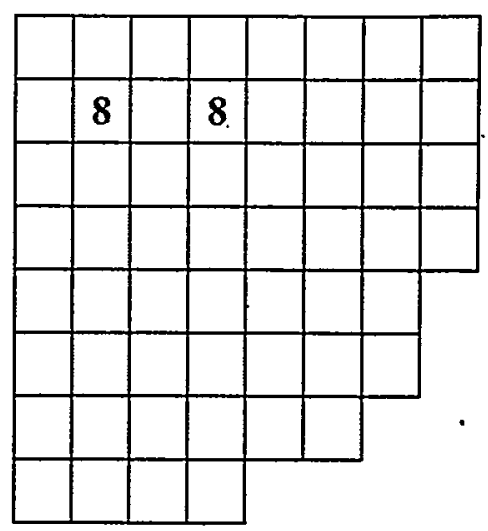

4.5-6.0 GWd/ST

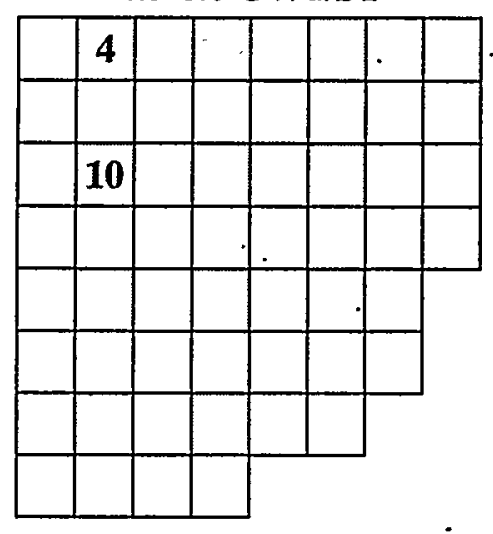

6.0-7.5 GWd/ST

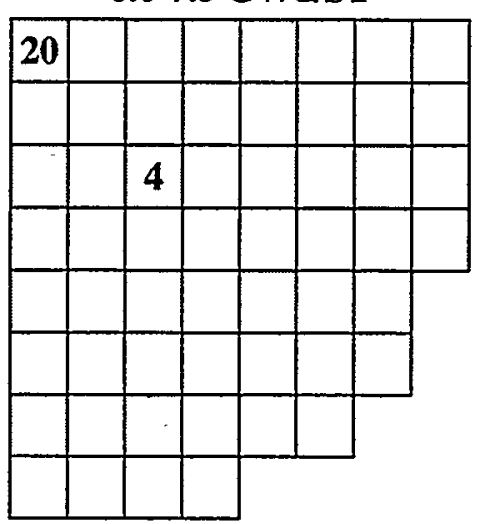

7.5-9.0 GWd/ST

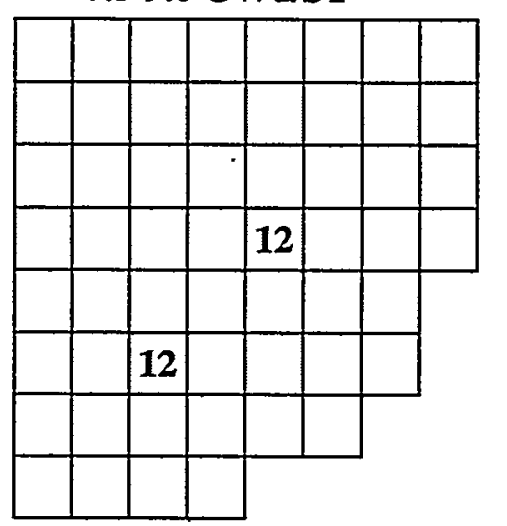

9.0-10.5 GWd/ST
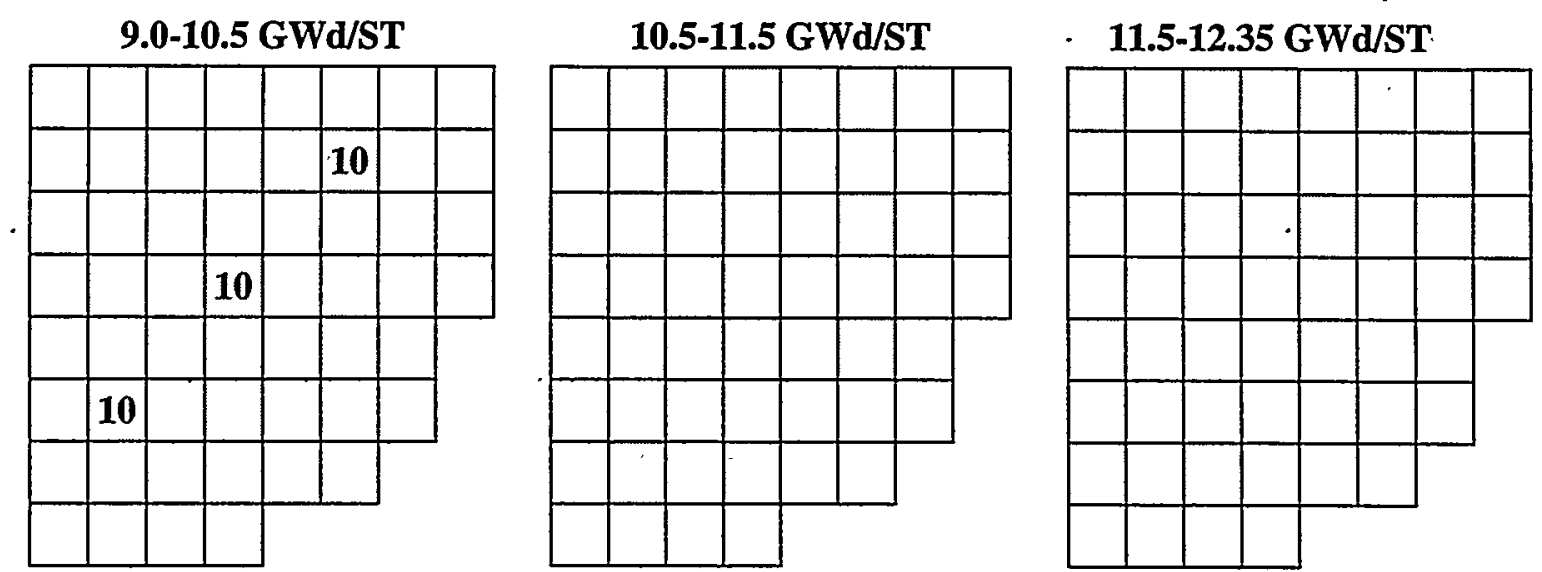

FIGURE 6.6. Scenario 1b (Optimized) Mixed MOX-LEU Core Control Rod Patterns 


\begin{tabular}{|c|c|c|c|c|c|c|c|c|c|c|c|c|c|c|c|}
\hline 26.44 & 15.05 & $\mid 13.97$ & 15.41 & $\mid 13.86$ & $\mid 15.64$ & 13.51 & 27.19 & 0.00 & 28.94 & 0.00 & 27.38 & 0.00 & 15.47 & 0.00 & 27.91 \\
\hline 15.31 & 0.00 & 28.75 & 0.00 & 27.28 & 0.00 & 27.99 & 0.00 & 29.94 & 0.00 & 26.55 & 0.00 & 23.56 & 0.00 & 11.26 & 30.19 \\
\hline 13.69 & 29.43 & 13.51 & 15.52 & 12.92 & 15.59 & 12.98 & 15.18 & 0.00 & 15.11 & 0.00 & 14.31 & 0.00 & 21.51 & 27.79 & \\
\hline 14.93 & 0.00 & 15.22 & 0.00 & 26.16 & 0.00 & 28.81 & 0.00 & 28.65 & 0.00 & 25.05 & 0.00 & 24.05 & 14.33 & 31.08 & \\
\hline 13.54 & 27.37 & 12.70 & 27.46 & 10.88 & 15.74 & 12.67 & 15.14 & 0.00 & 15.15 & 0.00 & 14.69 & 0.00 & 14.36 & 31.58 & \\
\hline 14.52 & 0.00 & 14.90 & 0.00 & 15.10 & 0.00 & 29.05 & 0.00 & 29.77 & 0.00 & 25.18 & 0.00 & 21.18 & 14.07 & 28.27 & \\
\hline 13.43 & 28.62 & 12.68 & 29.29 & 12.48 & |27.92 & 0.00 & 15.13 & 0.00 & 15.51 & 0.00 & 15.51 & 0.00 & 11.52 & 28.23 & \\
\hline 27.59 & 0.00 & 14.16 & 0.00 & 14.14 & 0.00 & 14.62 & 0.00 & 24.01 & 0.00 & 23.20 & 0.00 & 12.58 & 28.48 & & \\
\hline 0.00 & 27.82 & 0.00 & 29.11 & 0.00 & 30.06 & 0.00 & 26.07 & 0.00 & 14.37 & 0.00 & 15.04 & 28.37 & & & \\
\hline 27.99 & 0.00 & 15.03 & 0.00 & 14.72 & 0.00 & 13.53 & 0.00 & 14.02 & 0.00 & 19.01 & 13.57 & 27.80 & & & \\
\hline 0.00 & 26.70 & 0.00 & 25.18 & 0.00 & 25.67 & 0.00 & 24.09 & 0.00 & 19.25 & 0.00 & 8.95 & 28.83 & & & \\
\hline 27.45 & 0.00 & 13.98 & 0.00 & 14.50 & 0.00 & 15.02 & 0.00 & 15.50 & 14.24 & 8.82 & 26.61 & & & & \\
\hline 0.00 & 24.76 & 0.00 & 24.31 & 0.00 & 21.46 & 0.00 & 12.47 & 29.18 & 27.93 & 28.97 & & & & & \\
\hline 15.34 & 0.00 & 21.84 & 13.93 & 15.56 & 13.71 & 11.39 & 28.03 & & & & & & & & \\
\hline 0.00 & 11.05 & 29.20 & 30.86 & 27.62 & 28.22 & 29.09 & & & & & & & & & \\
\hline 28.07 & 30.11 & & & & & & & & & & & & & & \\
\hline
\end{tabular}

FIGURE 6.7. Scenario 1b Mixed MOX-LEU Core 2D BỌC Expọsure Map

\begin{tabular}{|l|c|c|c|c|c|c|c|c|c|c|c|c|c|c|c|}
\hline 24.76 & 11.39 & 13.98 & 13.43 & 13.71 & 28.62 & 14.24 & 27.59 & 0.00 & 27.92 & 0.00 & 21.46 & 0.00 & 12.98 & 0.00 & 23.20 \\
\hline 27.46 & 0.00 & 15.04 & 0.00 & 26.07 & 0.00 & 27.82 & 0.00 & 28.81 & 0.00 & 15.05 & 0.00 & 15.56 & 0.00 & 14.50 & 29.20 \\
\hline 13.69 & 26.70 & 13.54 & 12.48 & 12.92 & 23.56 & 14.93 & 15.50 & 0.00 & 11.05 & 0.00 & 21.18 & 0.00 & 15.03 & 26.55 & \\
\hline 15.52 & 0.00 & 14.16 & 0.00 & 27.19 & 0.00 & 27.91 & 0.00 & 27.45 & 0.00 & 26.16 & 0.00 & 15.41 & 15.18 & 27.79 \\
\hline 15.34 & 29.77 & 13.93 & 27.37 & 15.74 & 26.44 & 14.69 & 27.99 & 0.00 & 24.01 & 0.00 & 12.68 & 0.00 & 29.29 & 28.22 \\
\hline 13.57 & 0.00 & 28.23 & 0.00 & 10.88 & 0.00 & 27.62 & 0.00 & 27.28 & 0.00 & 12.47 & 0.00 & 13.51 & 19.01 & 27.38 \\
\hline 15.59 & 28.07 & 14.72 & 14.36 & 14.31 & 25.18 & 0.00 & 13.53 & 0.00 & 13.51 & 0.00 & 13.86 & 0.00 & 25.18 & 30.86 \\
\hline 27.80 & 0.00 & 15.15 & 0.00 & 15.64 & 0.00 & 15.31 & 0.00 & 14.52 & 0.00 & 28.97 & 0.00 & 25.67 & 15.51 & \\
\hline 0.00 & 28.37 & 0.00 & 15.22 & 0.00 & 28.03 & 0.00 & 15.11 & 0.00 & 29.05 & 0.00 & 12.67 & 28.83 & & \\
\hline 26.61 & 0.00 & 14.62 & 0.00 & 14.02 & 0.00 & 25.05 & 0.00 & 14.37 & 0.00 & 19.25 & 13.97 & 30.11 & & \\
\hline 0.00 & 27.93 & 0.00 & 15.51 & 0.00 & 14.90 & 0.00 & 29.94 & 0.00 & 8.82 & 0.00 & 12.58 & 29.09 & & \\
\hline 15.47 & 0.00 & 8.95 & 0.00 & 28.75 & 0.00 & 11.26 & 0.00 & 14.14 & 28.94 & 15.13 & 30.19 & & & \\
\hline 0.00 & 28.65 & 0.00 & 11.52 & 0.00 & 12.70 & 0.00 & 15.14 & 28.27 & 31.58 & 29.43 & & & & \\
\hline 14.07 & 0.00 & 21.51 & 14.33 & 24.05 & 15.02 & 24.31 & 28.48 & & & & & & & \\
\hline 0.00 & 15.10 & 29.18 & 29.11 & 31.08 & 30.06 & 24.09 & & & & & & & &
\end{tabular}

FIGURE 6.8. Scenario 1b (Optimized) Mixed MOX-LEU Core 2D BOC Exposure Map 


\subsubsection{Nearly Full MOX Core}

The core obtained by continuing the scenario $1 \mathrm{~b}$ mixed MOX-LEU core and assuming MOX feed assemblies to be available is referred to as the nearly full MOX core. The core contains eight thrice-burned LEU assemblies, with the rest of the burned assemblies being MOX assemblies. As before, the LP is obtained by using the shuffle instructions employed in the equilibrium cycle, full MOX core (Figure 4.3) and the CRP is obtained using FORMOSA-B's CRP search capability. Figure 6.9 shows the resulting CRP. The nearly full MOX core has acceptable thermal margin and core flow values as shown in Table 6.4.

TABLE 6.4: Nearly Full MOX Core's Key Attributes

\begin{tabular}{|c|cccccccc|}
\hline $\begin{array}{c}\text { Cycle } \\
\text { Exposure } \\
\text { (GWd/ST) }\end{array}$ & $\begin{array}{c}\text { \% Rated } \\
\text { Core } \\
\text { Flow }\end{array}$ & MFLPD & MAPRAT & MFLCPR & $\begin{array}{c}\text { MHGR } \\
\text { kw/ft. }\end{array}$ & MCPR & $\begin{array}{c}\text { 100\% Core } \\
\text { Flow) }\end{array}$ \\
\hline \hline 0 & 85.298 & 0.928 & $\cdot 0.853$ & 0.916 & 12.023 & 1.517 & 1.0185 \\
1.5 & 85.619 & 0.892 & 0.789 & 0.888 & 11.555 & 1.565 & 1.0199 \\
3.0 & 86.403 & $\mathbf{0 . 9 3 0}$ & 0.835 & 0.903 & 12.058 & 1.538 & 1.0163 \\
4.5 & 85.313 & 0.795 & 0.793 & 0.904 & 10.298 & 1.538 & 1.0137 \\
6.0 & 94.572 & 0.927 & $\mathbf{0 . 8 8 5}$ & 0.873 & 12.014 & 1.592 & 1.0125 \\
7.5 & 91.324 & 0.918 & 0.879 & 0.930 & 11.897 & 1.494 & 1.0126 \\
9.0 & 86.433 & 0.862 & 0.861 & 0.931 & 11.176 & 1.492 & 1.0120 \\
10.5 & 88.193 & 0.908 & 0.799 & 0.881 & 11.773 & 1.578 & 1.0085 \\
11.5 & 95.648 & 0.887 & 0.799 & 0.856 & 11.500 & 1.623 & 1.0042 \\
12.35 & $\mathbf{1 0 5 . 1 6 3}$ & 0.821 & 0.820 & 0.845 & 10.635 & 1.644 & 0.9995 \\
\hline
\end{tabular}



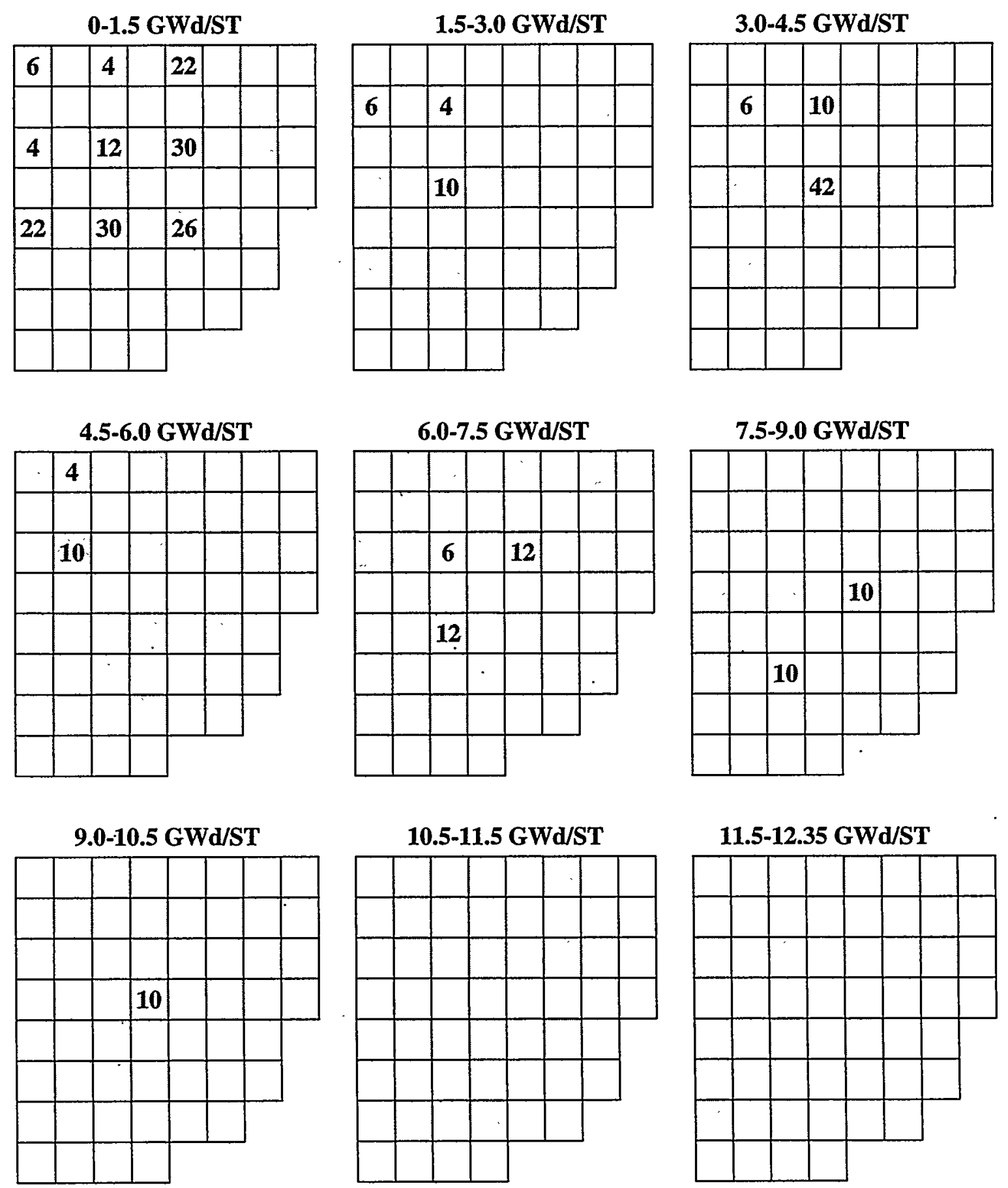

FIGURE 6.9. Nearly Full MOX Core Control Rod Patterns 


\subsubsection{Full MOX Core}

Continuing from the nearly full MOX core and assuming MOX feed assemblies continue to be available results in a full MOX core. The CRP is shown in Figure 6.10. Thermal margin and core flow values are judged to be acceptable (Table 6.5).

TABLE 6.5: Full MOX Core's Key Attributes

\begin{tabular}{|c|cccccccc|}
\hline $\begin{array}{c}\text { Cycle } \\
\text { Exposure } \\
\text { (GWd/ST) }\end{array}$ & $\begin{array}{c}\text { \% Rated } \\
\text { Core } \\
\text { Flow }\end{array}$ & MFLPD & MAPRAT & MFLCPR & $\begin{array}{c}\text { LHGR/f. } \\
\text { kwax. }\end{array}$ & MCPR & $\begin{array}{c}\text { 100\% Core } \\
\text { Flow) }\end{array}$ \\
\hline \hline 0 & 85.298 & 0.933 & 0.862 & 0.908 & 12.090 & 1.530 & 1.0185 \\
1.5 & 84.761 & 0.873 & 0.805 & 0.887 & 11.315 & 1.567 & 1.0200 \\
3.0 & 85.104 & 0.901 & 0.812 & 0.905 & 11.681 & 1.536 & 1.0164 \\
4.5 & 85.316 & 0.805 & 0.784 & 0.905 & 10.437 & 1.536 & 1.0137 \\
6.0 & 92.666 & $\mathbf{0 . 9 3 8}$ & 0.902 & 0.872 & 12.156 & 1.593 & 1.0125 \\
7.5 & 91.649 & 0.914 & 0.860 & 0.924 & 11.846 & 1.504 & 1.0125 \\
9.0 & 85.030 & 0.918 & 0.917 & 0.930 & 11.902 & 1.494 & 1.0118 \\
10.5 & 87.180 & 0.902 & 0.800 & 0.885 & 11.689 & 1.570 & 1.0083 \\
11.5 & 94.307 & 0.872 & 0.805 & 0.864 & 11.296 & 1.609 & 1.0042 \\
12.35 & $\mathbf{1 0 3 . 4 2 9}$ & 0.802 & 0.829 & 0.855 & 10.401 & 1.626 & 0.9997 \\
\hline
\end{tabular}

\subsection{Summary of Disruption Scenario 1 Cores}

We conclude that we have successfully established a disruption scenario in which the mixed MOX-LEU core is introduced by loading LEU fresh assemblies into the initial mixed core, and a full MOX core is produced by assuming the complete availability of the MOX fresh assemblies for subsequent cycles following the initial mixed MOX-LEU core. The MOX feed assemblies' mean enrichments (Table 6.6) are constant, since only one 
MOX fuel bundle design was utilized throughout this study. Figure 6.11 through Figure 6.17 summarizes disruption scenario 1 cores' key properties.
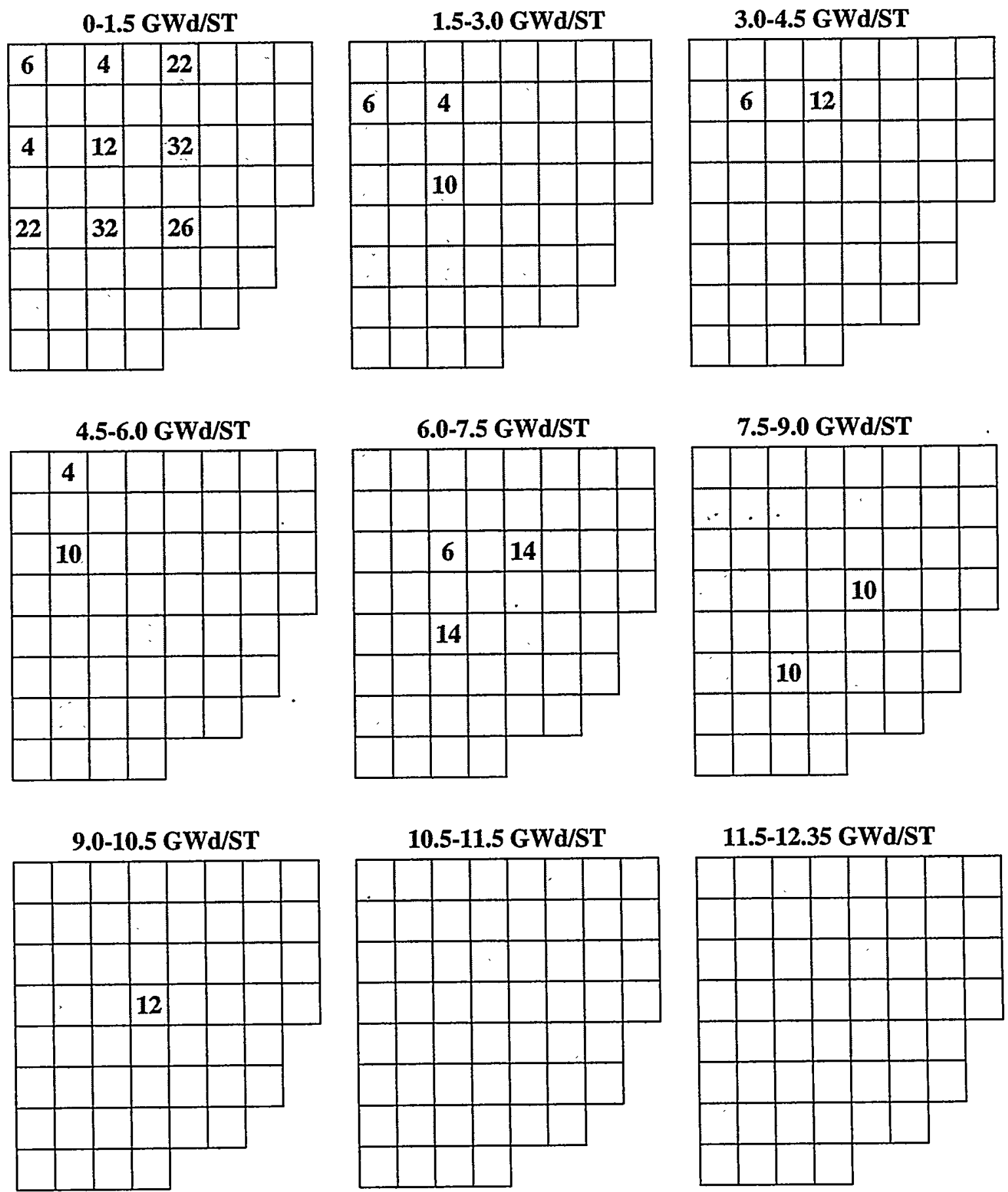

FIGURE 6.10. Full MOX Core Control Rod Patterns 
TABLE 6.6: Summary of Feed Assemblies' Mean Enrichment for Disruption Scenario 1 Cores

\begin{tabular}{|lc|}
\hline \multicolumn{1}{|c|}{ Core } & Mean Enrichment \\
\hline \hline Equilibrium Cycle, Full MOX Core & 4.310 w/o WGPu \\
Initial Mixed MOX-LEU Core & 3.847 w/o U-235 \\
Scenario 1 Mixed MOX-LEU Core & 4.310 w/o WGPu \\
Scenario 1b Mixed MOX-LEU Core & 4.310 w/o WGPu \\
Nearly Full MOX Core & 4.310 w/o WGPu \\
Full MOX Core & 4.310 w/o WGPu \\
\hline
\end{tabular}

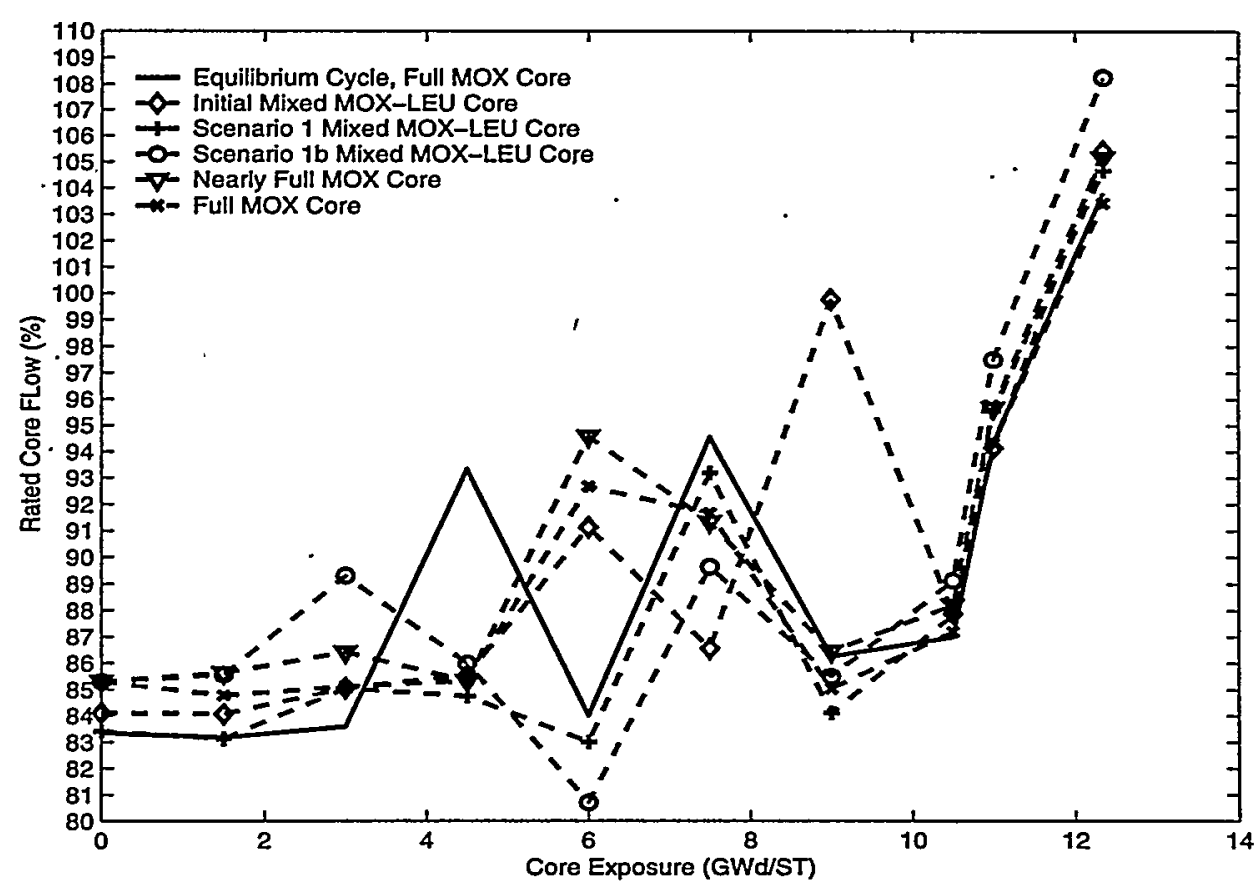

FIGURE 6.11. Summary of Rated Core Flow for Disruption Scenario 1 Cores 


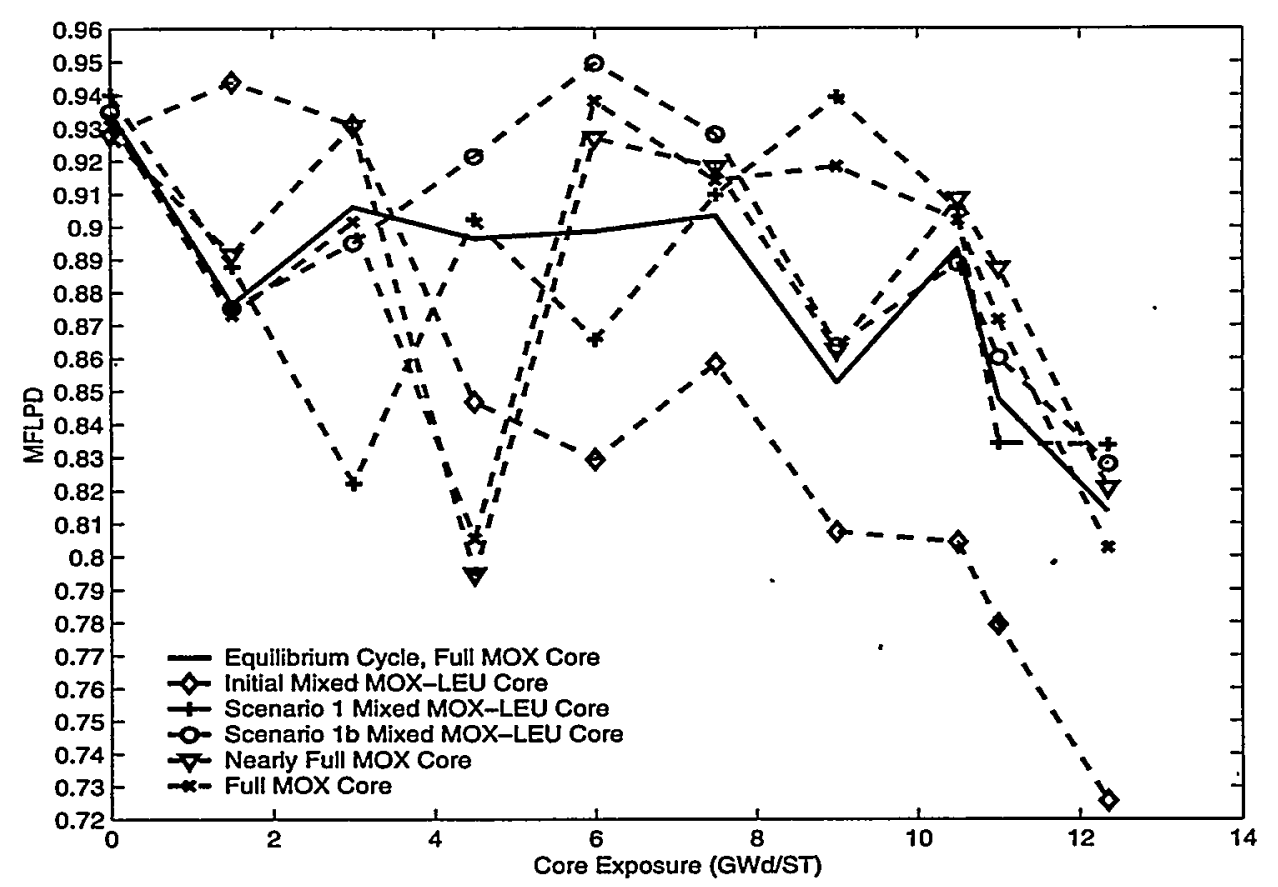

FIGURE 6.12. Summary of MFLPD for Disruption Scenario 1 Cores

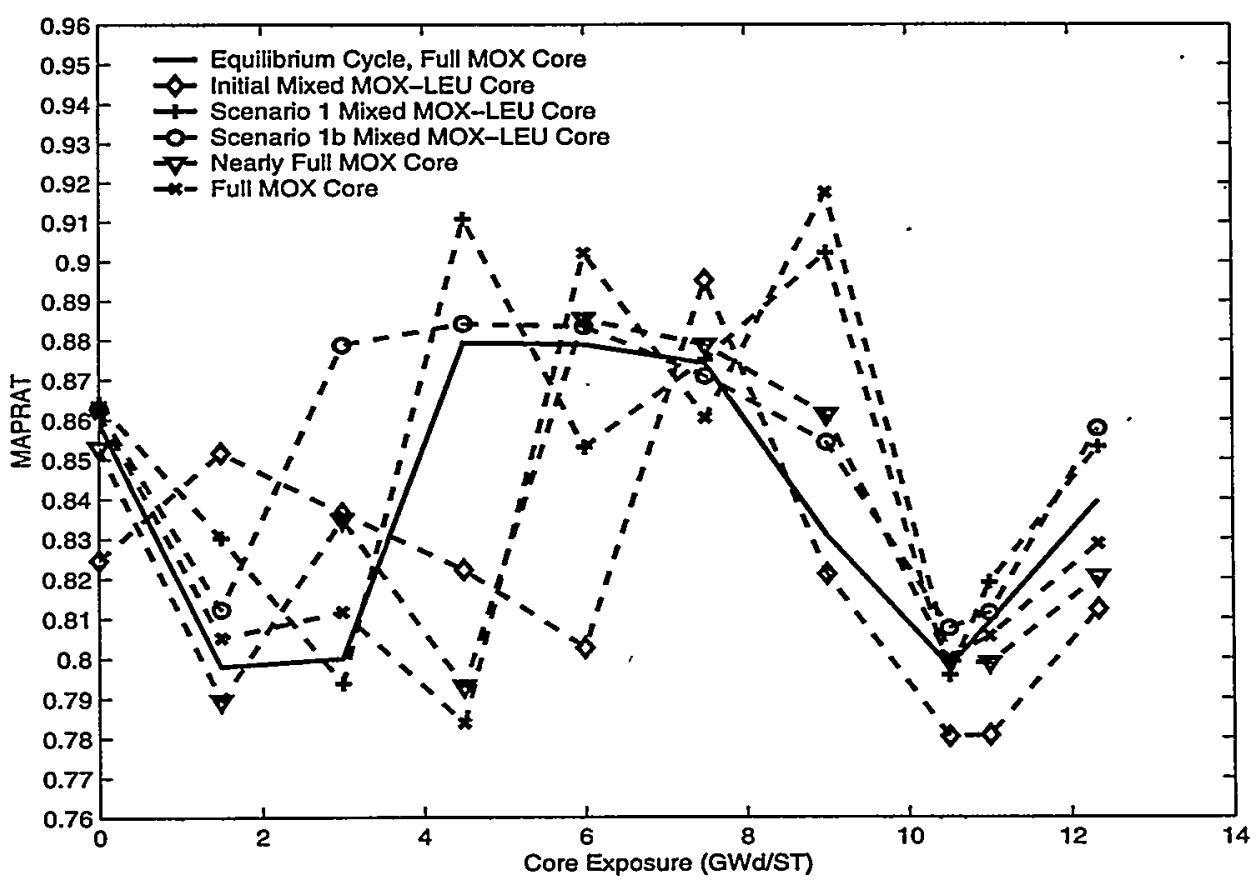

FIGURE 6.13. Summary of MAPRAT for Disruption Scenario 1 Cores 


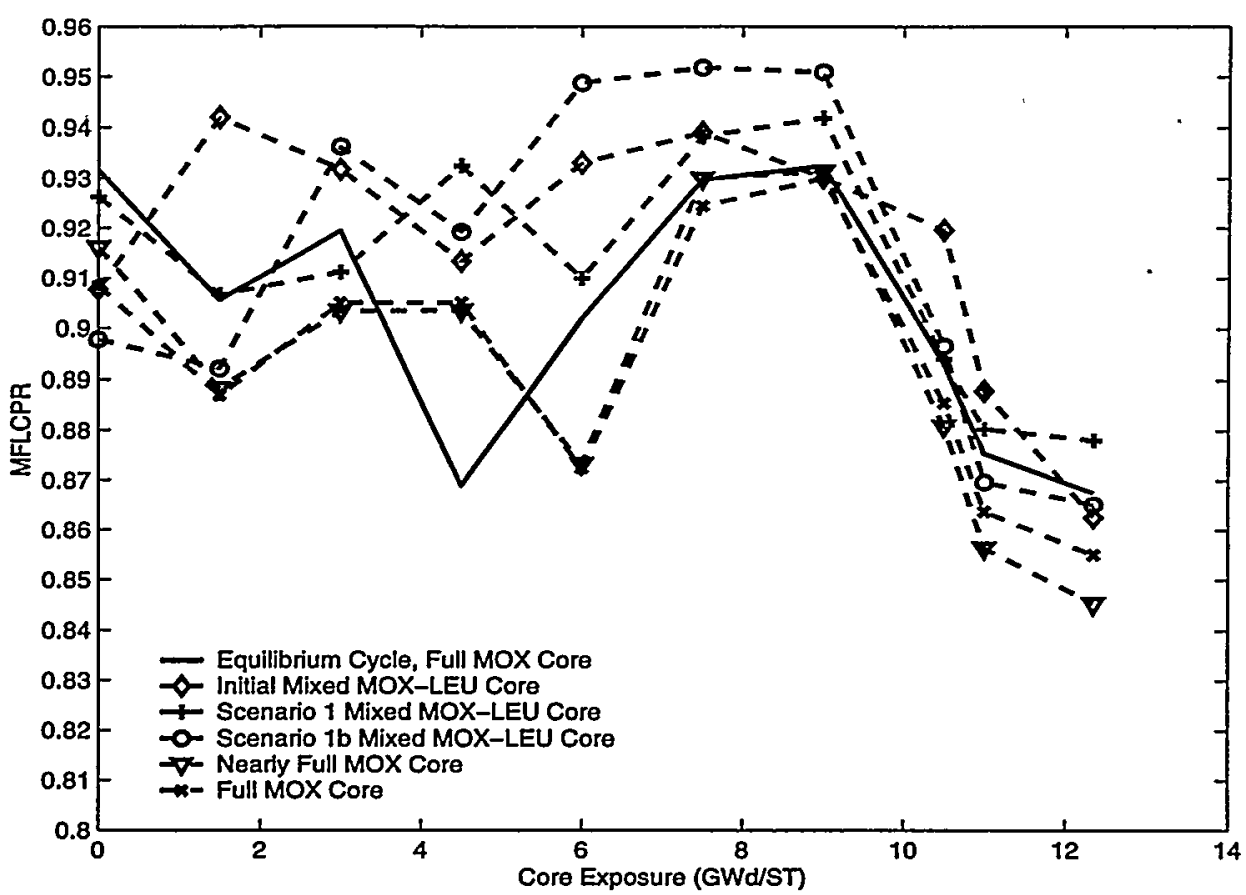

FIGURE 6.14. Summary of MFLCPR for Disruption Scenario 1 Cores

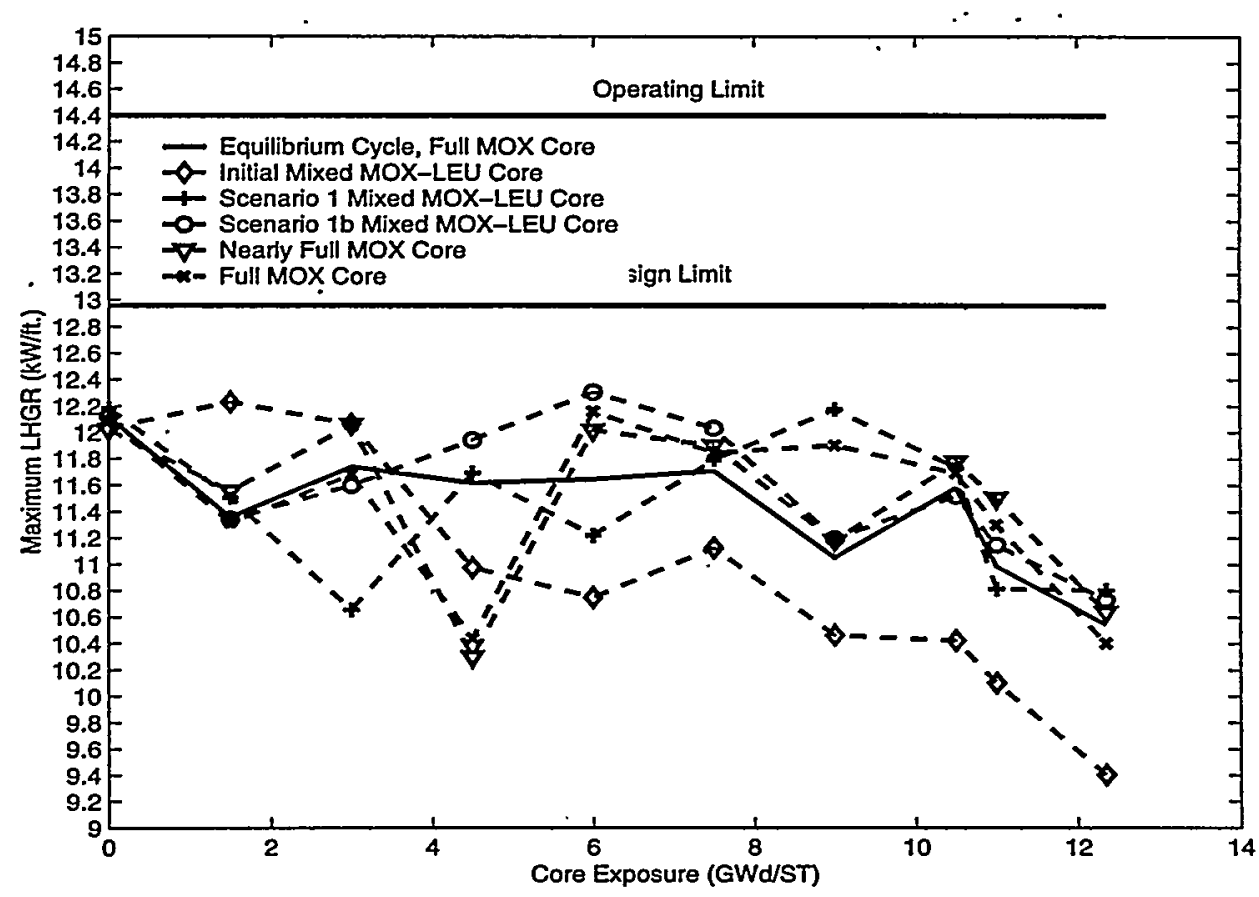

FIGURE 6.15. Summary of Maximum LHGR for Disruption Scenario 1 Cores 


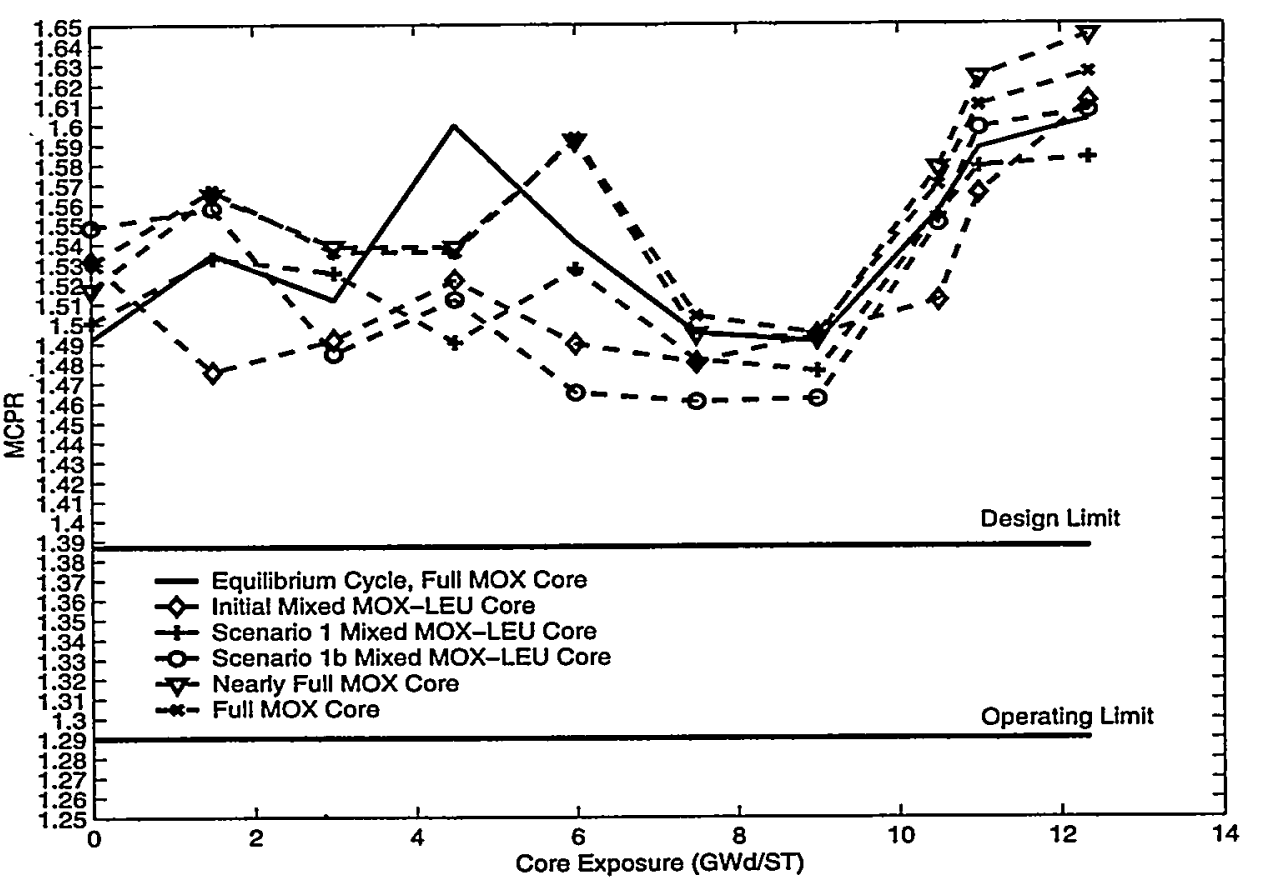

FIGURE 6.16. Summary of MCPR for Disruption Scenario 1 Cores

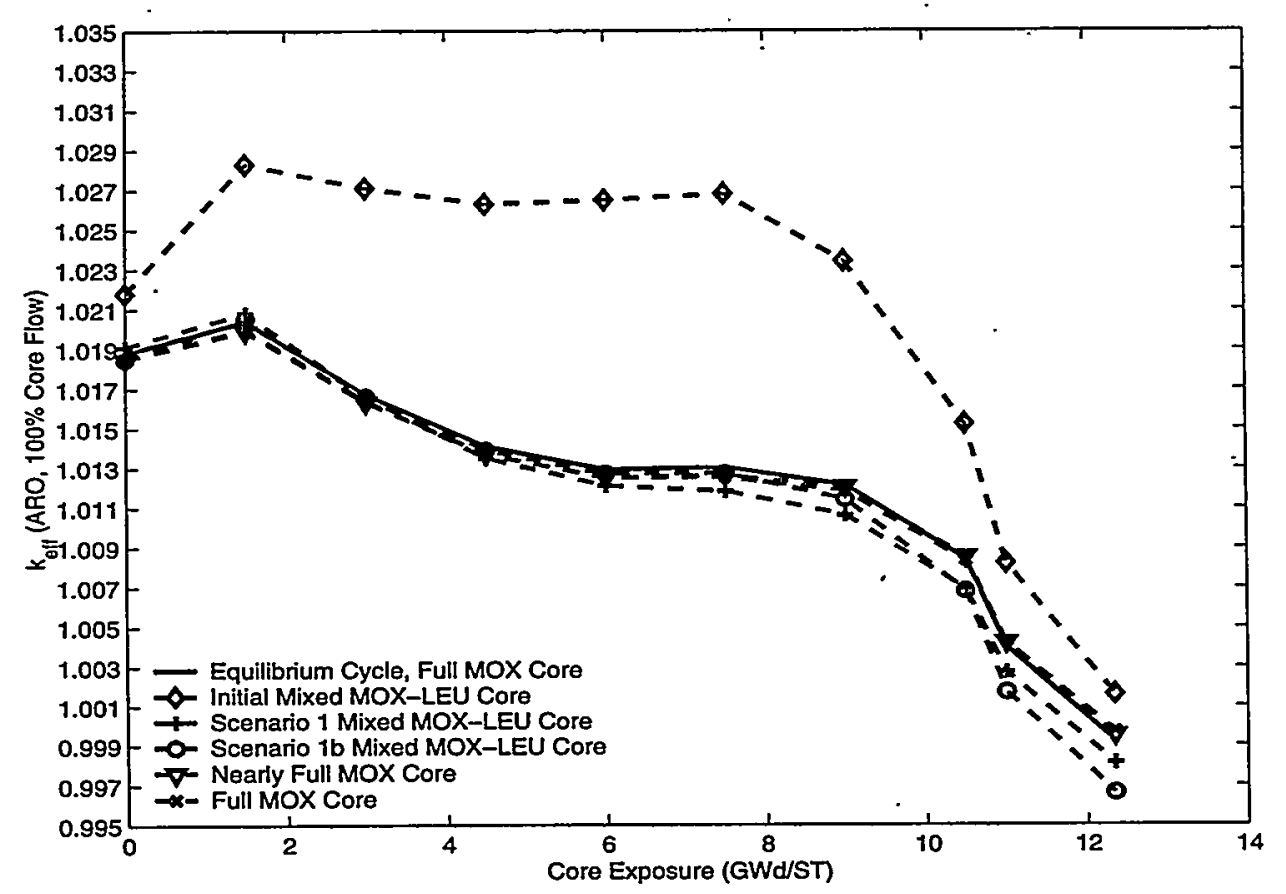

FIGURE 6.17. Summary of $k_{\text {eff }}$ (ARO, $100 \%$ Flow) for Disruption Scenario 1 Cores 


\subsection{Scenario 2: MOX Feed Batches Continue to be Unavailable for Subsequent Cycle}

Similar to the approach used in developing the initial mixed MOX-LEU core, the subsequent cycle's loading pattern is determined using the equilibrium cycle, full MOX core's shuffling instruction shown in Figure 4.3. Two enrichment types of LEU fresh assemblies (Figure 5.7 and Figure 5.8) are employed. The mix of LEU enrichment 2 and 3 is determined such that the cycle energy requirement is met. The CRP for the core is determined utilizing FORMOSA-B's CRP search capability with the core flow adjusted to meet the target $k_{\text {eff }}$. Again, the target $k_{\text {eff }}$ is kept at 1.000 throughout the cycle depletion. The core in which the second region of LEU feed assemblies is loaded will be referred to as the scenario 2 mixed MOX-LEU core.

The CRP and LP for the resulting scenario 2 mixed MOX-LEU core are shown in Figure 6.18 and Figure 6.19, respectively. The LEU feed assemblies' mean' enrichment for the scenario 2 mixed MOX-LEU core is higher than the mean enrichment required in the initial mixed MOX-LEU core. This occurs since lattice reactivity decreases faster with burnup for LEU versus MOX lattices. The core's key attributes are summarized in Table 6.7. Thermal limits and EOC core flow required to achieve criticality for the scenario 2 mixed MOX-LEU core are comparable to the values obtained for the equilibrium cycle, full MOX and the initial mixed MOX-LEU cores. It is noted that a slightly higher cycle maximum MFLCPR (0.963) value exists. Performing a FORMOSAB LP/CRP search $\left(2,000\right.$ histories of OSA local search with a $k_{\text {inf }}$ range of 0.18 and OSA objective of cycle length maximization) lowers the cycle maximum MFLCPR value to an acceptable 0.948 (Table 6.8). 
TABLE 6.7: Scenario 2 Mixed MOX-LEU Core's Key Attributes

\begin{tabular}{|c|c|c|c|c|c|c|c|}
\hline $\begin{array}{c}\text { Cycle } \\
\text { Exposure } \\
(\mathbf{G W d} / \mathrm{ST})\end{array}$ & $\begin{array}{c}\text { \% Rated } \\
\text { Core } \\
\text { Flow }\end{array}$ & MFLPD & MAPRAT & MFLCPR & $\begin{array}{c}\text { Max. } \\
\text { LHGR } \\
\text { kw/ft. }\end{array}$ & MCPR & $\begin{array}{c}\mathbf{k}_{\mathrm{eff}}(\mathrm{ARO}, \\
100 \% \text { Core } \\
\text { Flow) }\end{array}$ \\
\hline 0 & 84.098 & 0.932 & 0.833 & 0.916 & 12.085 & 1.518 & 1.0253 \\
\hline 1.5 & 82.181 & 0.935 & 0.863 & 0.956 & 12.124 & 1.455 & 1.0314 \\
\hline 3.0 & 83.019 & 0.909 & 0.817 & 0.934 & 11.777 & 1.488 & 1.0291 \\
\hline 4.5 & 88.442 & 0.908 & 0.879 & 0.934 & 11.771 & 1.488 & 1.0274 \\
\hline 6.0 & 90.677 & 0.921 & 0.850 & 0.915 & 11.933 & 1.520 & 1.0267 \\
\hline 7.5 & 88.293 & 0.886 & 0.913 & 0.963 & 11.489 & 1.444 & 1.0263 \\
\hline 9.0 & 84.305 & 0.826 & 0.843 & 0.961 & 10.709 & 1.440 & 1.0235 \\
\hline 10.5 & 85.683 & 0.808 & 0.776 & 0.907 & 10.478 & 1.532 & 1.0164 \\
\hline 11.5 & 93.641 & 0.819 & 0.785 & 0.862 & 10.620 & 1.613 & 1.0098 \\
\hline 12.35 & 105.422 & 0.802 & 0.829 & 0.855 & 10.401 & 1.626 & 0.9997 \\
\hline
\end{tabular}

TABLE 6.8: Scenario 2 (Optimized) Mixed MOX-LEU Core's Key Attributes

\begin{tabular}{|c|cccccccc|}
\hline $\begin{array}{c}\text { Cycle } \\
\text { Exposure } \\
\text { (GWd/ST) }\end{array}$ & $\begin{array}{c}\text { \% Rated } \\
\text { Core } \\
\text { Flow }\end{array}$ & MFLPD & MAPRAT & MFLCPR & $\begin{array}{c}\text { LFG. } \\
\text { kw/f. }\end{array}$ & MCPR & $\begin{array}{c}\text { 100\% Core } \\
\text { Flow) }\end{array}$ \\
\hline \hline 0 & 85.440 & $\mathbf{0 . 9 3 5}$ & 0.830 & 0.916 & 12.119 & 1.517 & 1.0239 \\
1.5 & 92.412 & 0.895 & 0.839 & 0.903 & 11.602 & 1.539 & 1.0300 \\
3.0 & 88.754 & 0.905 & 0.823 & 0.911 & 11.723 & 1.526 & 1.0278 \\
4.5 & 96.814 & 0.838 & 0.795 & 0.938 & 10.858 & 1.482 & 1.0261 \\
6.0 & 87.195 & 0.832 & 0.837 & 0.920 & 10.784 & 1.511 & 1.0253 \\
7.5 & 91.643 & 0.864 & 0.907 & 0.944 & 11.198 & 1.472 & 1.0250 \\
9.0 & 86.138 & 0.815 & 0.826 & $\mathbf{0 . 9 4 8}$ & 10.557 & 1.466 & 1.0225 \\
10.5 & 84.782 & 0.824 & 0.786 & 0.911 & 10.678 & 1.525 & 1.0159 \\
11.5 & 92.733 & 0.794 & 0.781 & 0.877 & 10.290 & 1.585 & 1.0095 \\
12.35 & $\mathbf{1 0 3 . 4 5 2}$ & 0.761 & 0.772 & 0.851 & 9.858 & 1.634 & 1.0033 \\
\hline
\end{tabular}


TABLE 6.9: Nearly Full LEU Core's Key Attributes

\begin{tabular}{|c|cccccccc|}
\hline $\begin{array}{c}\text { Cycle } \\
\text { Exposure } \\
\text { (GWd/ST) }\end{array}$ & $\begin{array}{c}\text { \% Rated } \\
\text { Core } \\
\text { Flow }\end{array}$ & MFLPD & MAPRAT & MFLCPR & $\begin{array}{c}\text { MHGR } \\
\text { kw/ft. }\end{array}$ & MCPR & $\begin{array}{c}\text { k } \\
\text { 100\% Core } \\
\text { Flow) }\end{array}$ \\
\hline \hline 0 & 84.098 & $\mathbf{0 . 9 3 4}$ & 0.830 & 0.905 & 12.104 & 1.536 & 1.0274 \\
$1.5 \cdot$ & 86.287 & 0.920 & 0.822 & 0.949 & 11.919 & 1.464 & 1.0337 \\
3.0 & 84.651 & 0.884 & 0.858 & 0.951 & 11.453 & 1.462 & 1.0311 \\
4.5 & 90.370 & 0.872 & 0.856 & 0.925 & 11.297 & 1.502 & 1.0289 \\
6.0 & 86.459 & 0.923 & 0.923 & 0.952 & 11.963 & 1.460 & 1.0277 \\
7.5 & 89.664 & 0.906 & 0.898 & 0.950 & 11.736 & 1.463 & 1.0269 \\
9.0 & 85.835 & 0.842 & 0.858 & 0.956 & 10.909 & 1.454 & 1.0239 \\
10.5 & 84.999 & 0.844 & 0.803 & 0.923 & 10.932 & 1.506 & 1.0169 \\
11.5 & 93.160 & 0.853 & 0.832 & 0.880 & 11.061 & 1.580 & 1.0102 \\
12.35 & $\mathbf{1 0 4 . 7 7 4}$ & 0.826 & 0.811 & 0.845 & 10.706 & 1.645 & 1.0037 \\
\hline
\end{tabular}

TABLE 6.10: Full LEU Core's Key Attributes

\begin{tabular}{|c|c|c|c|c|c|c|c|}
\hline $\begin{array}{c}\text { Cycle } \\
\text { Exposure } \\
\text { (GWd/ST) }\end{array}$ & $\begin{array}{c}\text { \% Rated } \\
\text { Core } \\
\text { - Hlow }\end{array}$ & MFLPD & MAPRAT & MFLCPR & $\begin{array}{c}\text { Max. } \\
\text { LHGR } \\
\text { kw/ft. }\end{array}$ & MCPR & $\begin{array}{c}\mathbf{k}_{\text {eff }}(\text { ARO, } \\
100 \% \text { Core } \\
\text { Flow) }\end{array}$ \\
\hline 0 & 85.245 & 0.936 & 0.831 & 0.912 & 12.127 & 1.524 & 1.0267 \\
\hline 1.5 & 85.089 & 0.922 & 0.851 & 0.921 & 11.945 & 1.508 & 1.0331 \\
\hline 3.0 & 85.980 & 0.906 & 0.815 & 0.927 & 11.748 & 1.499 & 1.0307 \\
\hline 4.5 & 91.279 & 0.832 & 0.810 & 0.940 & 10.786 & 1.479 & 1.0287 \\
\hline 6.0 & 86.230 & 0.803 & 0.762 & 0.928 & 10.411 & 1.497 & 1.0277 \\
\hline 7.5 & 91.349 & 0.882 & 0.853 & 0.939 & 11.433 & 1.481 & 1.0270 \\
\hline 9.0 & 92.772 & 0.793 & $0.794^{\circ}$ & 0.939 & 10.281 & 1.480 & 1.0239 \\
\hline 10.5 & 84.996 & 0.827 & 0.802 & 0.931 & 10.714 & 1.492 & 1.0164 \\
\hline 11.5 & 92.678 & 0.830 & 0.807 & 0.883 & 10.756 & 1.574 & 1.0095 \\
\hline 12.35 & 103.704 & 0.793 & 0.767 & 0.855 & 10.282 & 1.626 & 1.0029 \\
\hline
\end{tabular}


First Ind ex

\begin{tabular}{|c|c|c|c|c|c|c|c|c|c|c|c|c|c|c|c|c|}
\hline & 16 & 15 & 14 & 13 & 12 & 11 & 10 & 9 & 8 & 7 & 6 & 5 & 4 & 3 & 2 & 1 \\
\hline 16 & 2 & 1 & 1 & 1 & 1 & 2 & 1 & 2 & F3 & 2 & F3 & 2 & F3 & 1 & F3 & 2 \\
\hline 15 & 2 & $\mathrm{~F} 2$ & 1 & $\mathrm{~F} 2$ & 2 & F2 & 2 & F3 & 2 & F3 & 1 & F3 & 1 & F3 & 1 & 2 \\
\hline 14 & 1 & 2 & 1 & 1 & 1 & 2 & 1 & 1 & $\mathrm{~F} 2$ & 1 & F3 & 2 & F3 & 1 & 2 & \\
\hline 13 & 1 & $\mathrm{~F} 2$ & 1 & F3 & 2 & F3 & 2 & F3 & 2 & F3 & 2 & F3 & 1 & 1 & 2 & \\
\hline 12 & 1 & 2 & 1 & 2 & 1 & 2 & 1 & 2 & F3 & 2 & F3 & 1 & F2 & 2 & 2 & \\
\hline 11 & 1 & F2 & 2 & F3 & 1 & F3 & 2 & F3 & 2 & F3 & 1 & F3 & 1 & 1 & 2 & \\
\hline 10 & 1 & 2 & 1 & 1 & 1 & 2 & F3 & 1 & F3 & 1 & F3 & 1 & F2 & 2 & 2 & \\
\hline 9 & 2 & F3 & 1 & F3 & 1 & F3 & 1 & F3 & 1 & $\mathrm{~F} 2$ & 2 & F2 & 2 & 1 & & \\
\hline 8 & F3 & 2 & F2 & 1 & F3 & 2 & F3 & 1 & F3 & 2 & $\mathrm{~F} 2$ & 1 & 2 & & & \\
\hline 7 & 2 & F3 & 1 & F3 & 1 & F3 & 2 & F2 & 1 & F3 & 2 & 1 & 3 & & & \\
\hline 6 & F3 & 2 & F3 & 1 & F3 & 1 & F3 & 2 & F2 & 1 & $\mathrm{~F} 2$ & 1 & 2 & & & \\
\hline 5 & 1 & F3 & 1 & F3 & 2 & F3 & 1 & $\mathrm{~F} 2$ & 1 & 2 & 1 & 3 & & & & \\
\hline 4 & F3 & 2 & F3 & 1 & $\mathrm{~F} 2$ & 1 & F2 & 1 & 2 & 2 & 2 & & & & & \\
\hline 3 & 1 & F3 & 2 & 1 & 2 & 1 & 2 & 2 & & & & & & & & \\
\hline 2 & F3 & 1 & 2 & 2 & 2 & 2 & 2 & & & & & & & & & \\
\hline 1 & 2 & 2 & & & & & & & & & & & & & & \\
\hline
\end{tabular}

The numbers represent the number of previous cycles the bundle has been in the core. The symbol $\mathrm{F}$ denotes fresh fuels.

F2 = LEU Enrichment 2 (Figure 5.7)

F3 = LEU Enrichment 3 (Figure 5.8)

FIGURE 6.18. Scenario 2 Mixed MOX-LEU Core Loading Pattern 
0-1.5 GWd/ST

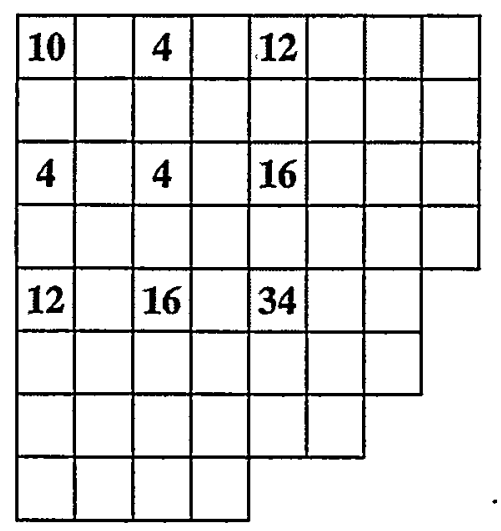

4.5-6.0 GWd/ST

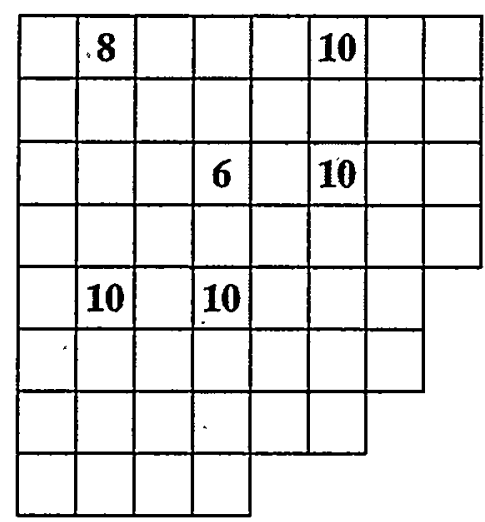

9.0-10.5 GWd/ST

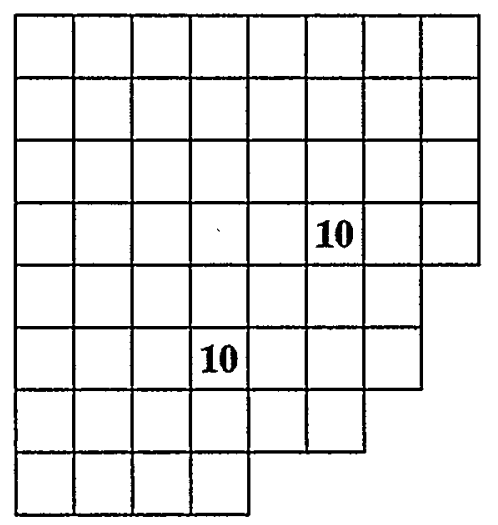

1.5-3.0 GWd/ST

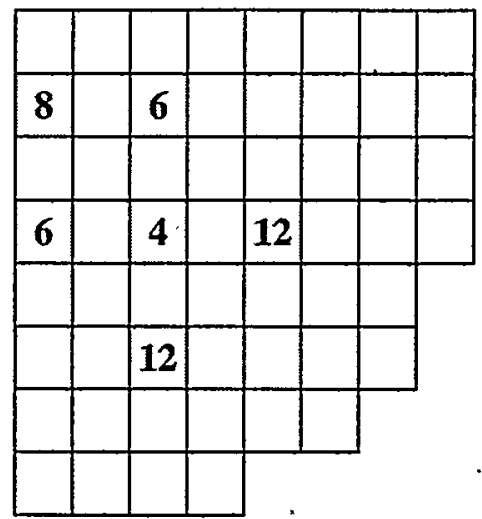

6.0-7.5 GWd/ST

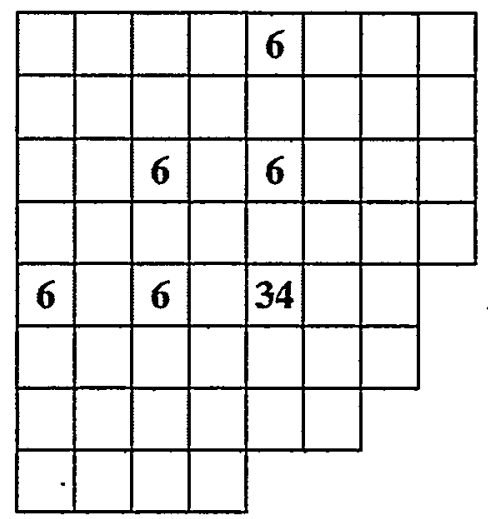

10.5-11.5 GWd/ST

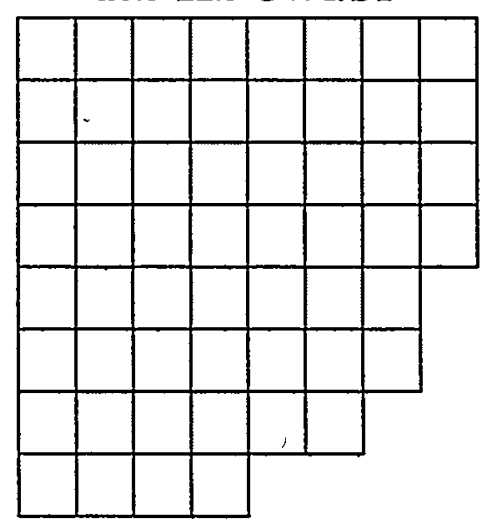

3.0-4.5 GWd/ST

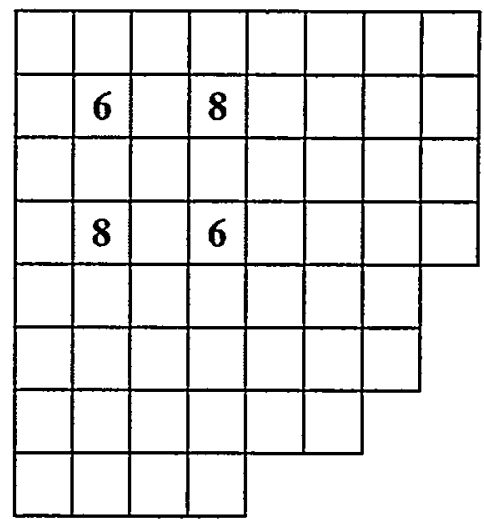

7.5-9.0 GWd/ST

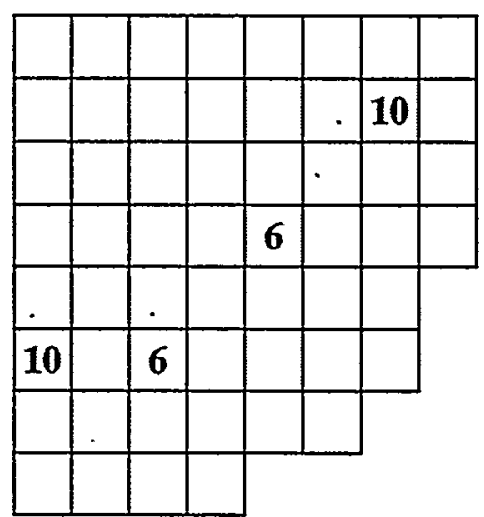

11.5-12.35 GWd/ST

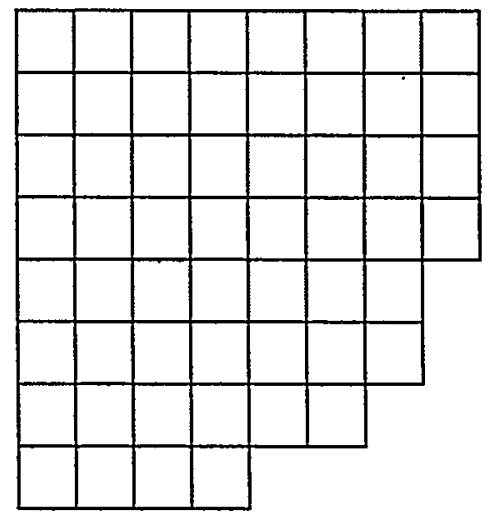

FIGURE 6.19. Scenario 2 Mixed MOX-LEU Core Control Rod Patterns 

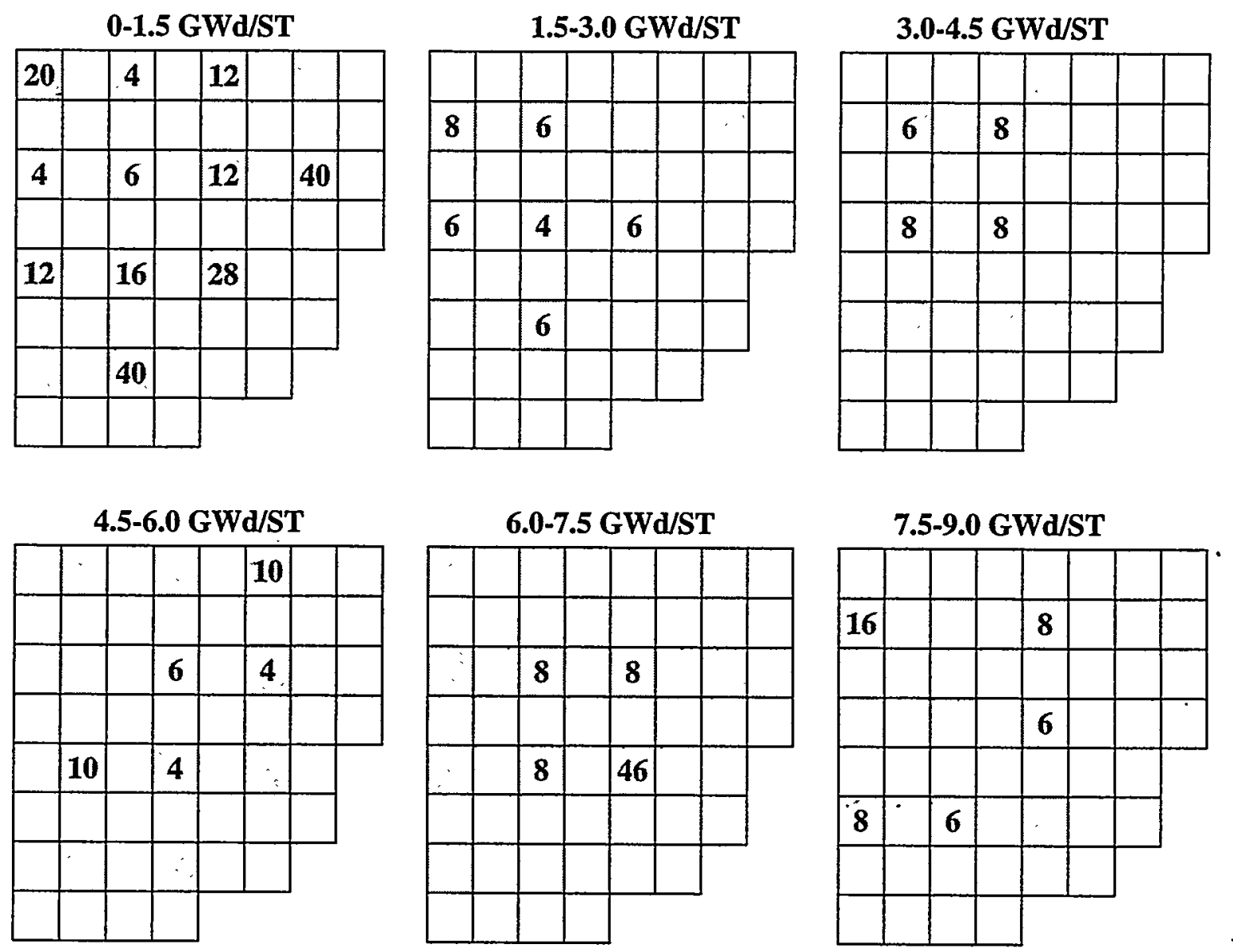

9.0-10.5 GWd/ST .

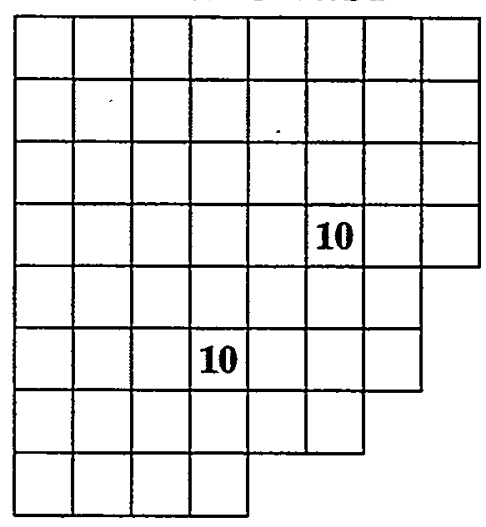

10.5-11.5 GWd/ST

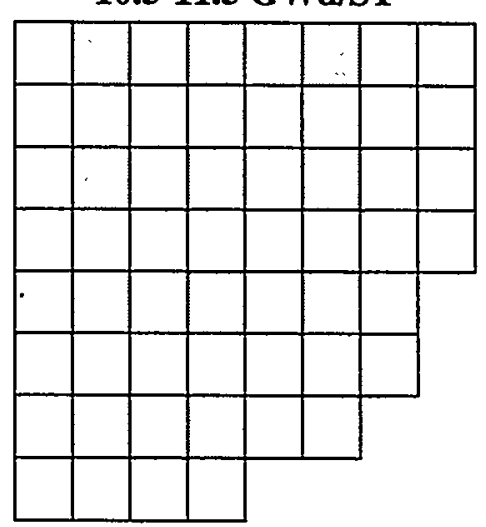

11.5-12.35 GWd/ST

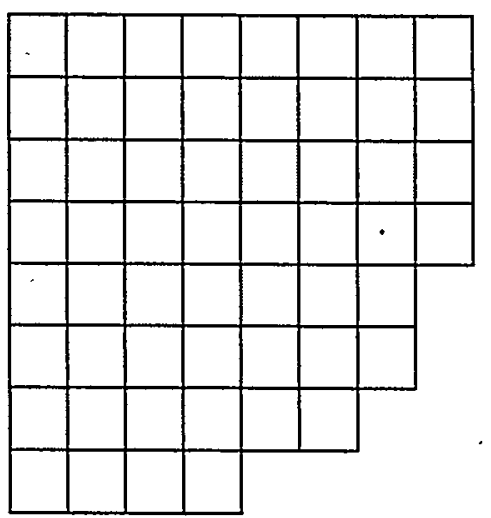

FIGURE 6.20. Scenario 2 (Optimized) Mixed MOX-LEU Core Control Rod Patterns 


\begin{tabular}{|c|c|c|c|c|c|c|c|c|c|c|c|c|c|c|c|}
\hline 24.10 & 14.14 & 14.80 & 13.29 & 15.43 & 13.81 & 14.91 & 28.27 & 0.00 & 29.64 & 0.00 & 27.75 & 0.00 & 13.80 & \begin{tabular}{|l|}
0.00 \\
\end{tabular} & 28.87 \\
\hline 14.29 & 0.00 & 28.60 & 0.00 & 27.85 & 0.00 & 26.50 & 0.00 & 27.06 & 0.00 & 26.74 & 0.00 & 24.30 & 0.00 & 12.26 & 30.48 \\
\hline 15.36 & 29.03 & 13.25 & 14.68 & 13.96 & 13.00 & 13.26 & 16.31 & 0.00 & 16.17 & 0.00 & 13.67 & 0.00 & 20.82 & 28.62 & \\
\hline 13.75 & 0.00 & 15.68 & 0.00 & 28.95 & 0.00 & 26.52 & 0.00 & 27.41 & 0.00 & 27.18 & 0.00 & 23.30 & 14.72 & 28.46 & \\
\hline 13.57 & 28.48 & 14.36 & 29.32 & 12.26 & 13.19 & 13.61 & 14.14 & 0.00 & 13.76 & 0.00 & 15.02 & 0.00 & 13.88 & 27.94 & \\
\hline 14.53 & 0.00 & 13.53 & 0.00 & 14.89 & 0.00 & 26.28 & 0.00 & 27.48 & 0.00 & 25.20 & 0.00 & 20.75 & 13.71 & 28.81 & \\
\hline 15.11 & 27.32 & 14.28 & 26.82 & 14.09 & 26.25 & 0.00 & 13.98 & $0: 00$ & 13.71 & 0.00 & 13.19 & 0.00 & 12.60 & 29.39 & \\
\hline 28.36 & 0.00 & 13.10 & 0.00 & 14.87 & 0.00 & 14.53 & 0.00 & 24.27 & 0.00 & 24.39 & 0.00 & 13.75 & 28.82 & & \\
\hline 0.00 & 27.02 & 0.00 & 27.39 & 0.00 & 27.20 & 0.00 & 26.22 & 0.00 & 14.64 & 0.00 & 15.28 & 29.68 & & & \\
\hline 29.61 & 0.00 & 16.48 & 0.00 & 14.20 & 0.00 & 13.75 & 0.00 & 13.67 & 0.00 & 18.47 & 13.50 & 28.42 & & & \\
\hline 0.00 & 27.70 & 0.00 & 27.71 & 0.00 & 25.34 & 0.00 & 24.50 & 0.00 & 18.41 & 0.00 & 10.10 & 28.12 & & & \\
\hline 28.65 & 0.00 & 14.15 & 0.00 & 16.01 & 0.00 & 14.76 & 0.00 & 15.49 & $\mid 14.78$ & 10.35 & 26.68 & & & & \\
\hline 0.00 & 24.98 & 0.00 & 23.65 & 0.00 & 20.89 & 0.00 & 14.16 & 28.39 & 28.56 & 27.55 & & & & & \\
\hline 14.14 & 0.00 & 21.09 & 15.33 & 16.00 & 14.61 & 12.98 & 29.29 & & & & & & & & \\
\hline 0.00 & 12.69 & 29.37 & 29.08 & 27.72 & 28.67 & 29.11 & & & & & & & & & \\
\hline .55 & 30.43 & & & & & & & & & & & & & & \\
\hline
\end{tabular}

FIGURE 6.21. Scenario 2 Mixed MOX-LEU Core 2D BOC Exposure Map

\begin{tabular}{|c|c|c|c|c|c|c|c|c|c|c|c|c|c|c|c|}
\hline 13.75 & 27.75 & 14.09 & 14.80 & 13.61 & 13.10 & 24.50 & 28.65 & 0.00 & 29.11 & 0.00 & 28.36 & 0.00 & 13.81 & 0.00 & 14.29 \\
\hline 27.18 & 0.00 & 14.14 & 0.00 & 13.29 & 0.00 & 27.71 & 0.00 & 26.25 & 0.00 & 26.28 & 0.00 & 27.70 & 0.00 & 12.26 & 29.61 \\
\hline 20.75 & 28.48 & 13.00 & 24.30 & 16.17 & 15.43 & 13.80 & 14.76 & 0.00 & 14.87 & 0.00 & 13.19 & 0.00 & 28.55 & 24.39 & \\
\hline 14.78 & 0.00 & 16.01 & 0.00 & 28.42 & 0.00 & 29.39 & 0,00 & 14.61 & 0.00 & 27.94 & 0.00 & 15.49 & 26.82 & 29.08 & \\
\hline 27.20 & 13.75 & 15.68 & 29.03 & 14.53 & 14.89 & 20.82 & 26.52 & 0.00 & 18.41 & 0.00 & 27.39 & 0.00 & 15.36 & 30.48 & \\
\hline 10.35 & 0.00 & 13.26 & 0.00 & 15.02 & 0.00 & 27.41 & 0.00 & 25.20 & 0.00 & 28.62 & \begin{tabular}{|l|}
0.00 \\
\end{tabular} & 15.33 & 24.10 & 20.89 & \\
\hline 14.68 & 28.95 & 13.71 & 14.14 & 13.57 & 15.28 & 0.00 & 24.27 & 0.00 & 14.36 & 0.00 & 14.16 & 0.00 & 12.69 & 16.00 & \\
\hline 28.12 & 0.00 & 14.15 & 0.00 & 13.96 & 0.00 & 28.46 & 0.00 & 16.48 & 0.00 & 13.98 & 0.00 & 12.26 & 28.87 & & \\
\hline 0.00 & 24.98 & 0.00 & 28.60 & 0.00 & 26.22 & 0.00 & 27.55 & 0.00 & 13.25 & 0.00 & 14.53 & 29.64 & & & \\
\hline 27.85 & 0.00 & 13.50 & 0.00 & 27.48 & 0.00 & 14.20 & 0.00 & 13.88 & 0.00 & 14.64 & 14.91 & 29.32 & & & \\
\hline 0.00 & 27.02 & 0.00 & 27.06 & 0.00 & 26.68 & 0.00 & 13.67 & 0.00 & 28.27 & 0.00 & 14.28 & 25.34 & & & \\
\hline 28.82 & 0.00 & 21.09 & 0.00 & 13.19 & 0.00 & 13.53 & 0.00 & 16.31 & 10.10 & 23.65 & 28.81 & & & & \\
\hline 0.00 & 14.72 & 0.00 & 12.98 & 0.00 & 13.67 & 0.00 & 13.75 & 28.39 & 18.47 & 29.37 & & & & & \\
\hline 23.30 & 0.00 & 13.71 & 13.76 & 26.74 & 14.14 & 15.11 & 27.72 & & & & & & & & \\
\hline 0.00 & 12.60 & 28.56 & 28.67 & 26.50 & 27.32 & 29.68 & & & & & & & & & \\
\hline 29.29 & 30.43 & & & & & & & & & & & & & & \\
\hline
\end{tabular}

FIGURE 6.22. Scenario 2 (Optimized) Mixed MOX-LEU Core 2D BOC Exposure Map 


\subsection{Transition Back to Full LEU Core}

\subsubsection{Nearly Full LEU Core}

Continuing from the scenario 2 mixed MOX-LEU core, which assumes that MOX assemblies are unavailable, another mixed MOX-LEU core is produced. This core is referred to as the nearly full LEU core since only eight out of 800 assemblies are MOX assemblies. The LP and CRP are shown in Figure 6.23 and Figure 6.24, respectively. A summary of thermal margin and core flow values are shown in Figure 6.9.

\subsubsection{Full LEU Core}

A full LEU core is obtained by continuing the nearly full LEU core and loading another region of LEU fresh fuel assemblies into the core. The LP and CRP are shown in Figure 6.25 and Figure 6.26. Thermal margin and core flow values are judged to be acceptable (Table 6.10). 
First Index

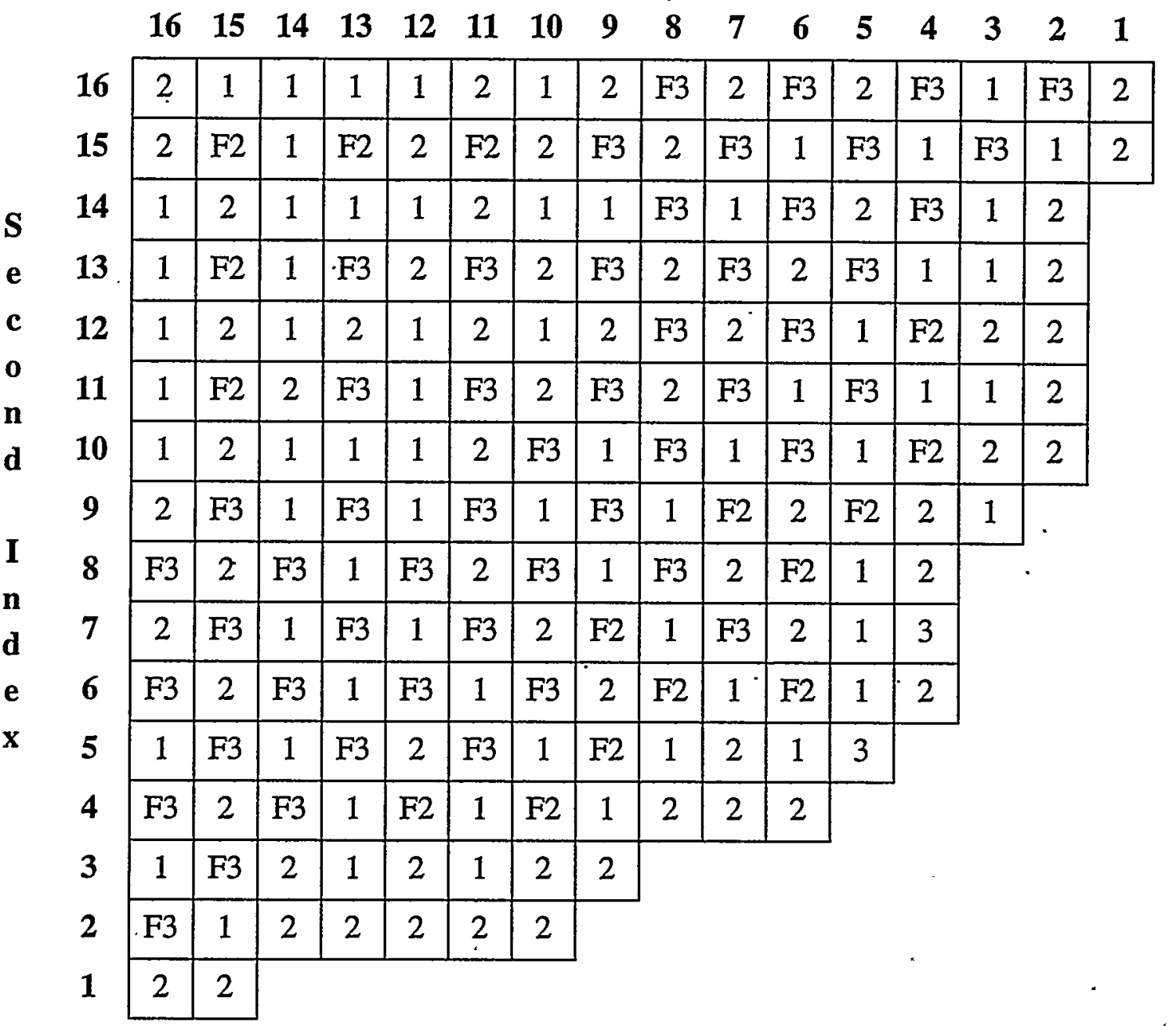

The numbers represent the number of previous cycles the bundle has been in the core. The symbol $\mathrm{F}$ denotes fresh fuels.

F2 = LEU Enrichment 2 (Figure 5.7)

F3 = LEU Enrichment 3 (Figure 5.8)

FIGURE 6.23. Nearly Full LEU Core Loading Pattern 
0-1.5 GWd/ST

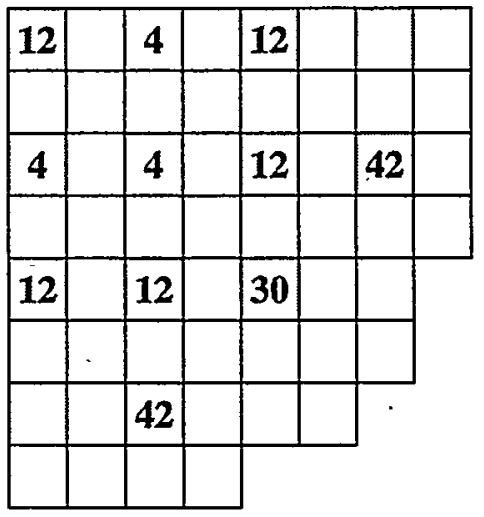

4.5-6.0 GWd/ST

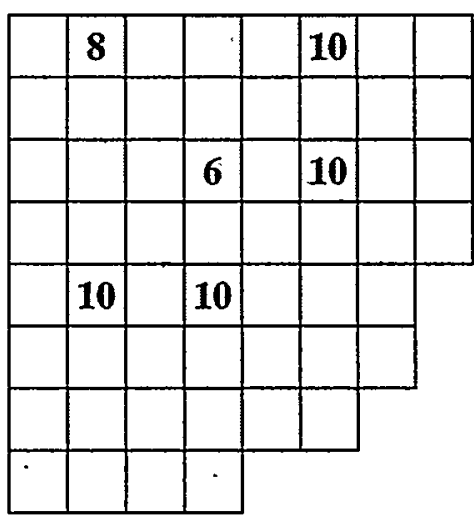

9.0-10.5 GWd/ST

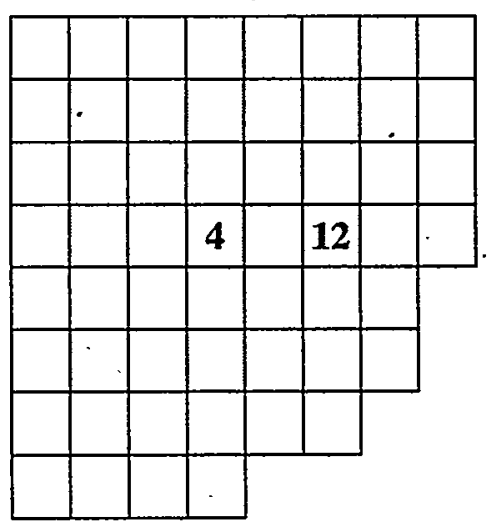

1.5-3.0 GWd/ST

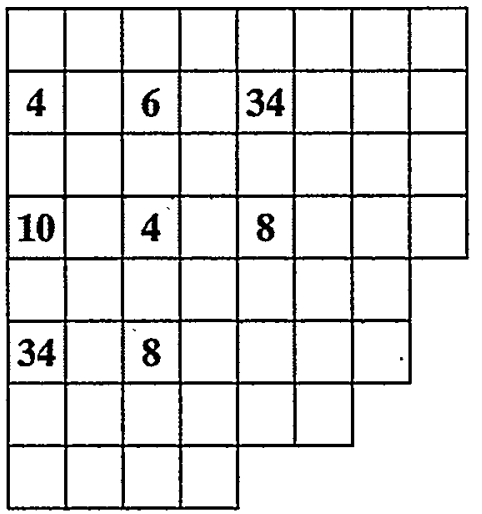

6.0-7.5 GWd/ST

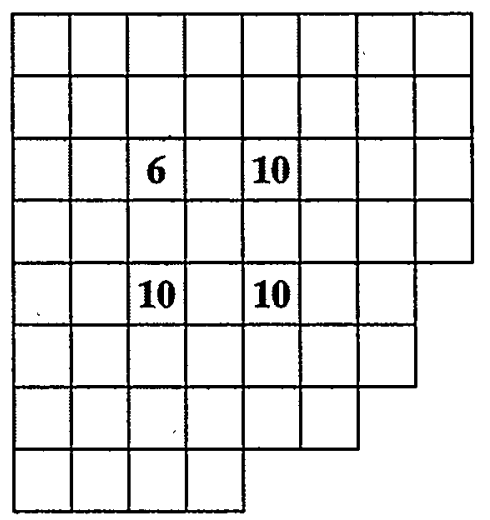

10.5-11.5 GWd/ST

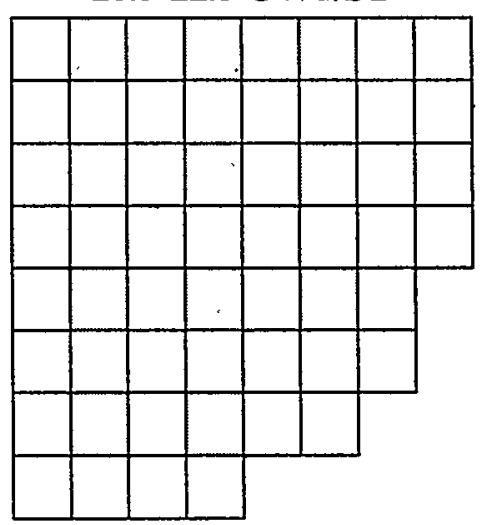

3.0-4.5 GWd/ST

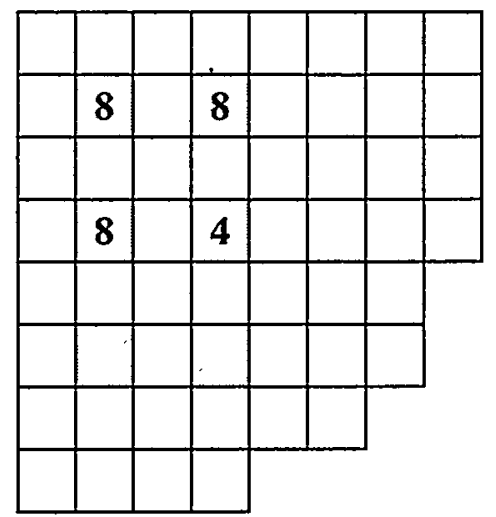

7.5-9.0 GWd/ST

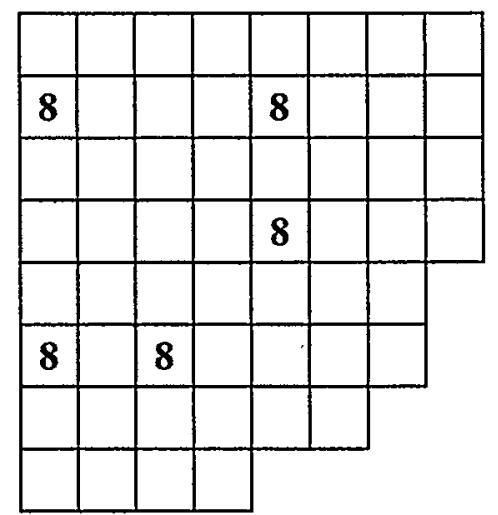

11.5-12.35 GWd/ST

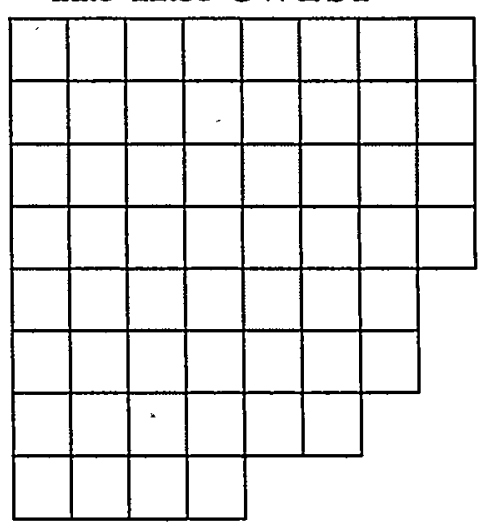

FIGURE 6.24. Nearly Full LEU Core Control Rod Patterns 
First Index

\begin{tabular}{|c|c|c|c|c|c|c|c|c|c|c|c|c|c|c|c|c|}
\hline & 16 & 15 & 14 & 13 & 12 & 11 & 10 & 9 & 8 & 7 & 6 & 5 & 4. & 3 & 2 & 1 \\
\hline 16 & 2 & 1 & 1 & 1 & 1 & 2 & 1 & 2 & F3 & 2 & F3 & 2 & F3 & 1 & F3 & 2 \\
\hline 15 & 2 & F2 & 1 & F2 & 2 & F2 & 2 & F3 & 2 & F3 & 1 & F3 & 1 & F3 & 1 & 2 \\
\hline 14 & 1 & 2 & 1 & 1 & 1 & 2 & 1 & 1 & F3 & 1 & F3 & 2 & F3 & 1 & 2 & \\
\hline 13 & 1 & F2 & 1 & F3 & 2 & F3 & 2 & F3 & 2 & F3 & 2 & F3 & 1 & 1 & 2 & \\
\hline 12 & 1 & 2 & 1 & 2 & 1 & 2 & 1 & 2 & F3 & 2 & F3 & 1 & F2 & 2 & 2 & \\
\hline 11 & 1 & F2 & 2 & F3 & 1 & F3 & 2 & F3 & 2 & F3 & 1 & F3 & 1 & 1 & 2. & \\
\hline 10 & 1 & 2 & 1 & 1 & 1 & 2 & F3 & 1 & F3 & 1 & F3 & 1 & F2 & 2 & 2 & \\
\hline 9 & 2 & F3 & 1 & F3 & 1 & F3 & 1 & F3 & 1 & $\mathrm{~F} 2$ & 2 & F2 & 2 & 1 & & \\
\hline 8 & F3 & 2 & F3 & 1 & F3 & 2 & F3 & 1 & F3 & 2 & $F 2$ & 1 & 2 & & & \\
\hline 7 & 2 & F3 & 1 & F3 & 1 & F3 & 2 & F2 & 1 & F3 & 2 & 1 & 3 & & & \\
\hline 6 & F3 & 2 & F3 & 1 & F3 & 1 & F3 & 2 & F2 & 1 & F2 & 1 & 2 & & & \\
\hline 5 & 1 & F3 & 1 & F3 & 2 & F3 & 1 & F2 & 1 & 2 & 1 & 3 & & & & \\
\hline 4 & F3 & 2 & F3 & 1 & F2 & 1 & F2 & 1 & 2 & 2 & 2 & & & & & \\
\hline 3 & 1 & F3 & 2 & 1 & 2 & 1 & 2 & 2 & & & & & & & & \\
\hline 2 & F3 & 1 & 2 & 2 & 2 & 2 & 2 & & . & & & & & & & \\
\hline 1 & 2 & 2 & & & & & & & & & & & & & & \\
\hline
\end{tabular}

The numbers represent the number of previous cycles the bundle has been in the core. The symbol $\mathrm{F}$ denotes fresh fuels.

F2 = LEU Enrichment 2 (Figure 5.7)

F3 = LEU Enrichment 3 (Figure 5.8)

FIGURE 6.25. Full LEU Core Loading Pattern 

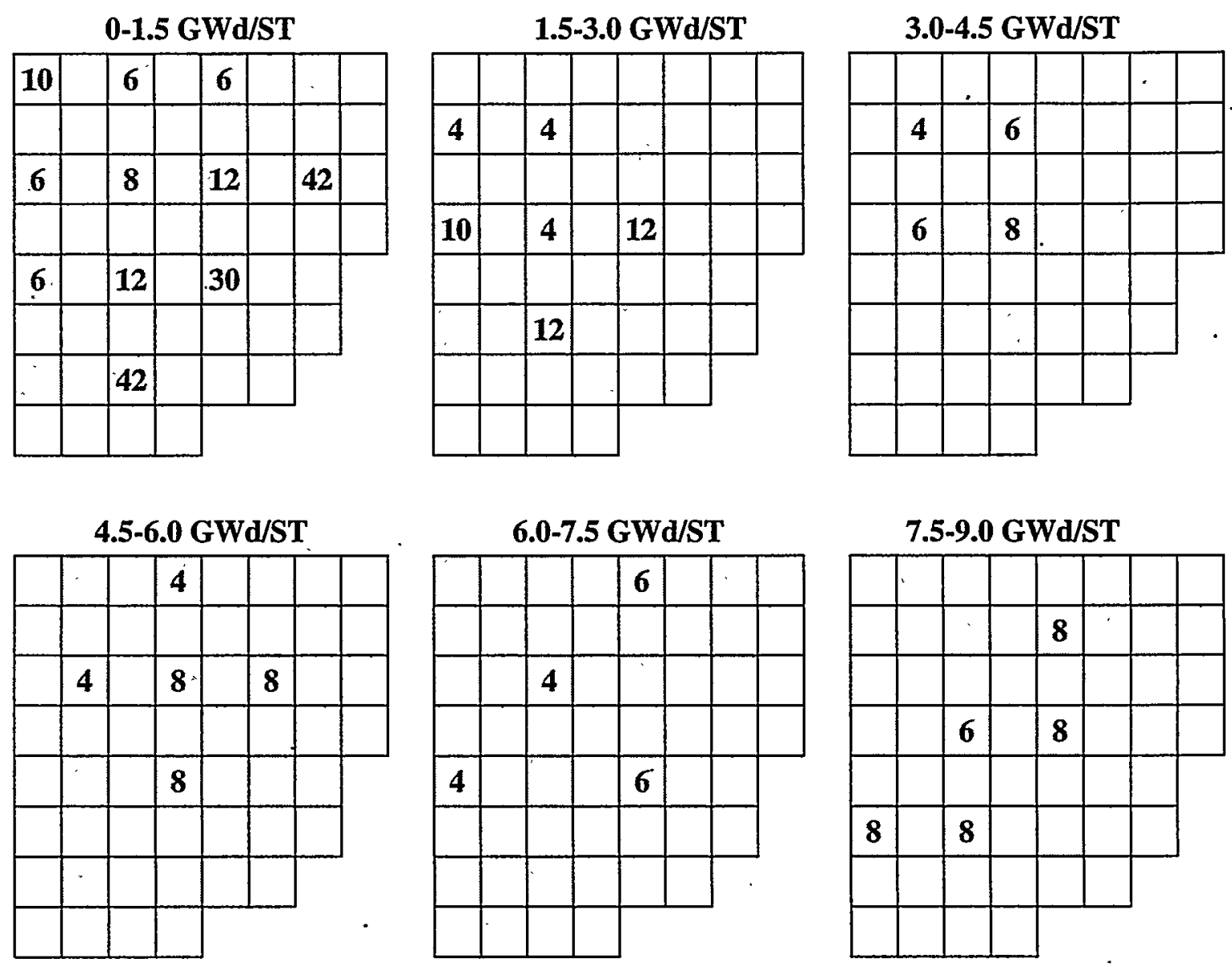

9.0-10.5 GWd/ST
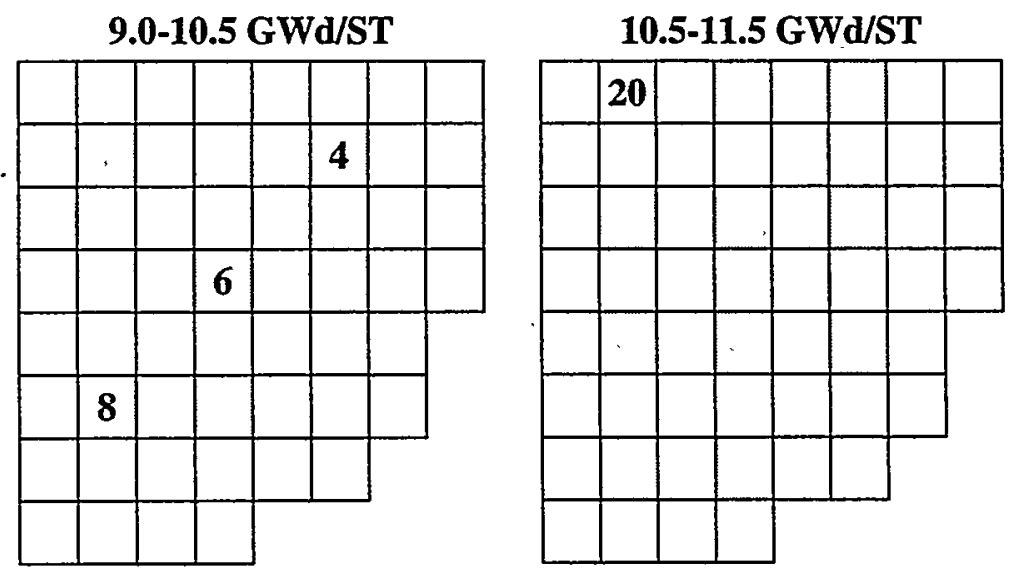

11.5-12.35 GWd/ST

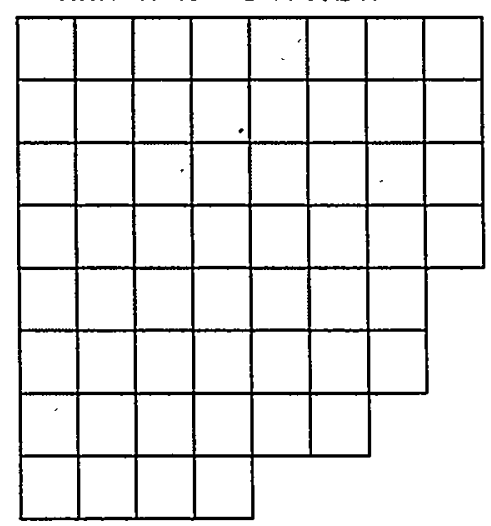

FIGURE 6.26. Full LEU Core Control Rod Patterns 


\subsection{Summary of Disruption Scenario 2 Cores}

We conclude that we have established the second disruption scenario in which the transitioning from an equilibrium cycle, full MOX core to a full LEU core occurs by assuming complete availability of LEU fresh assemblies for subsequent cycles following the initial mixed MOX-LEU core. A summary of LEU feed assemblies' mean enrichments is shown in Table 6.11. Figure 6.11 through Figure 6.17 summarizes disruption scenario 1 cores' key properties.

TABLE 6.11: Summary of Feed Assemblies' Mean Enrichment for Disruption Scenario 2 Cores

\begin{tabular}{|lc|}
\hline \multicolumn{1}{|c|}{ Core } & Mean Enrichment \\
\hline \hline Equilibrium Cycle, Full MOX Core & 4.310 w/o WGPu \\
Initial Mixed MOX-LEU Core & 3.847 w/o U-235 \\
Scenario 2 Mixed MOX-LEU Core & 3.915 w/o U-235 \\
Nearly Full LEU Core & 3.924 w/o U-235 \\
Full LEU Core & 3.870 w/o U-235 \\
\hline
\end{tabular}

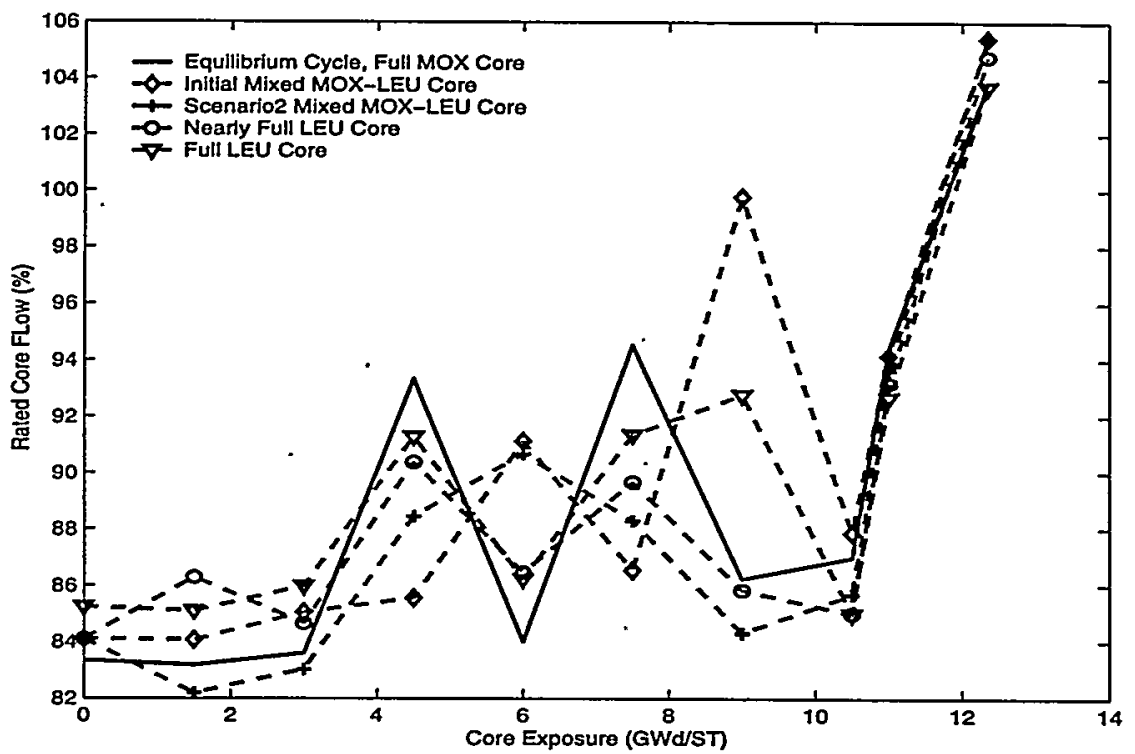

FIGURE 6.27. Summary of Rated Core Flow for Disruption Scenario 2 Cores 


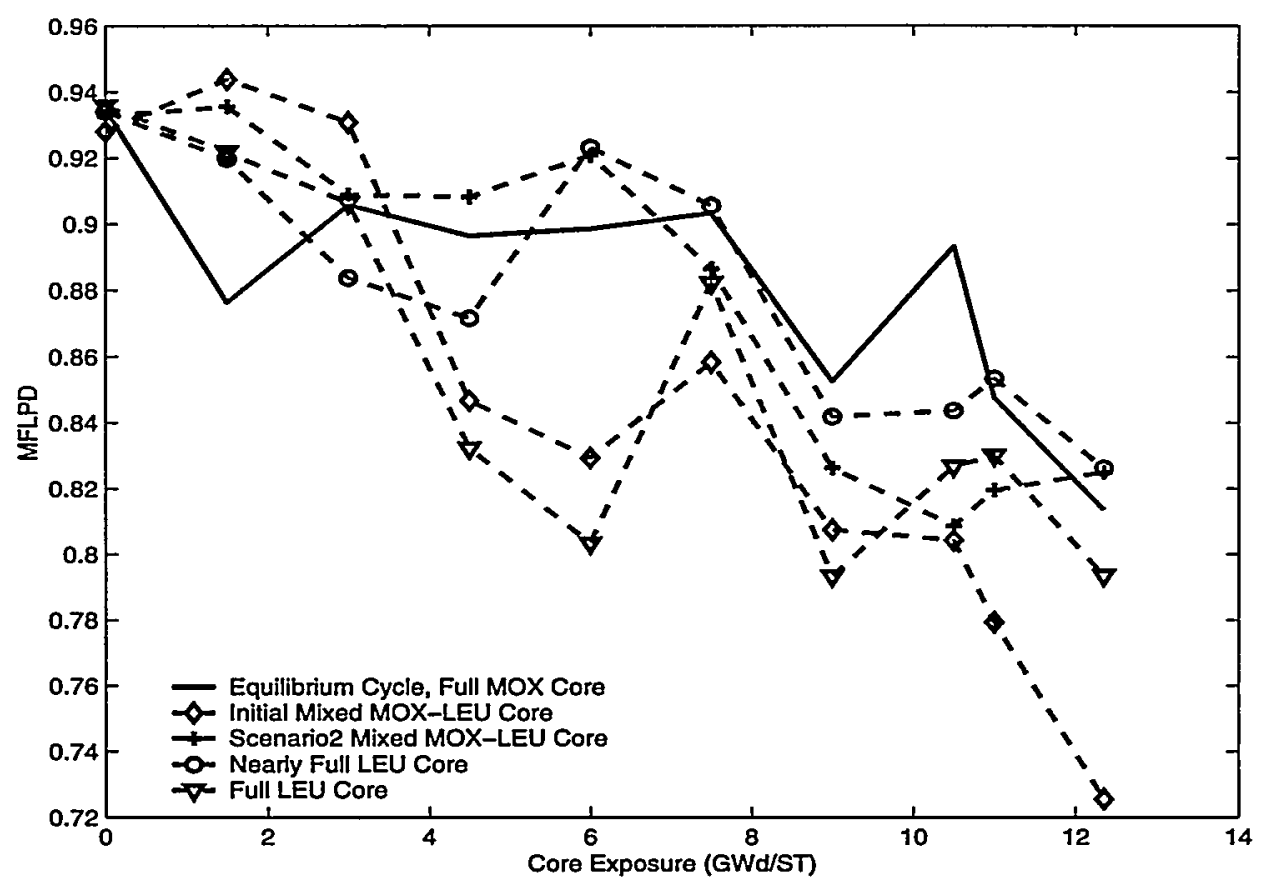

FIGURE 6.28. Summary of MFLPD for Disruption Scenario 2 Cores

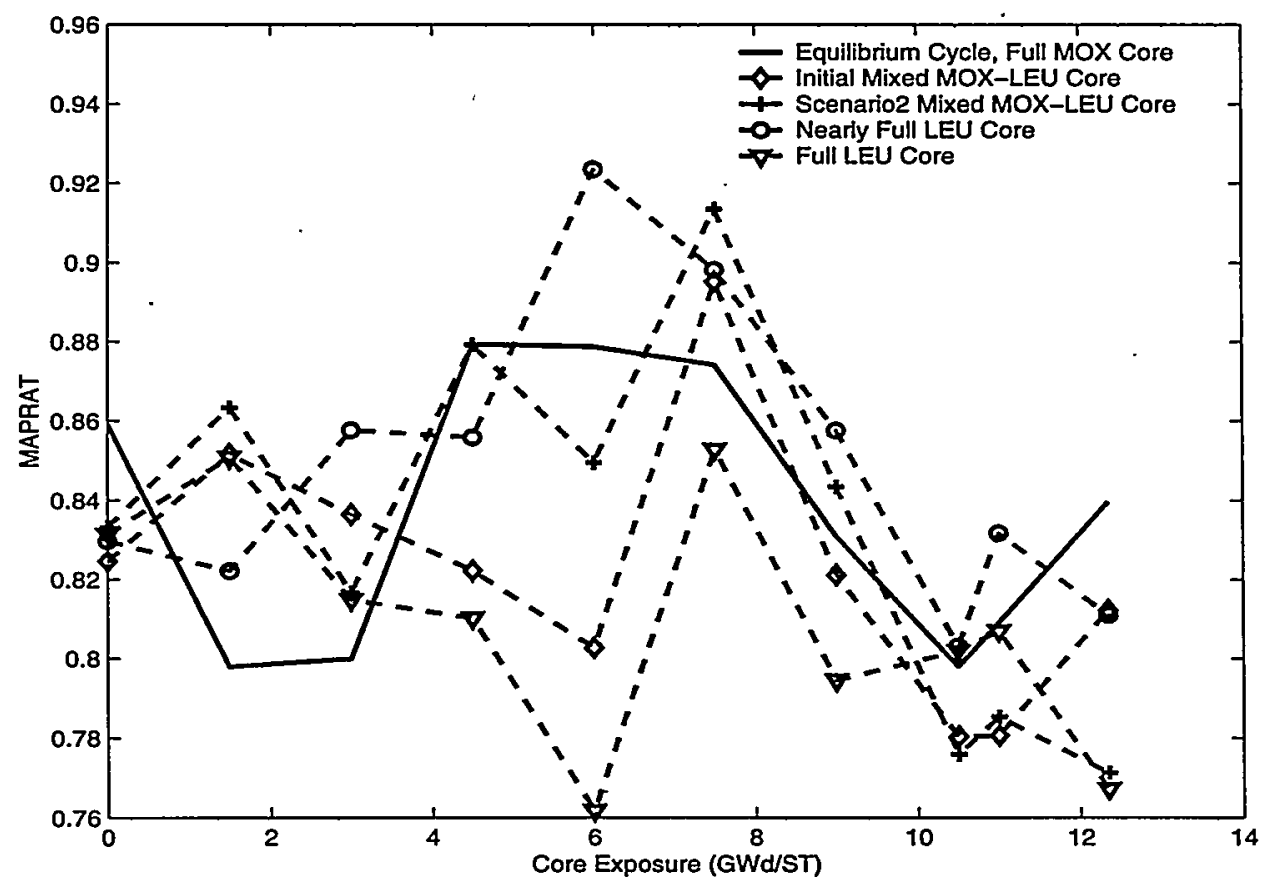

FIGURE 6.29. Summary of MAPRAT for Disruption Scenario 2 Cores 


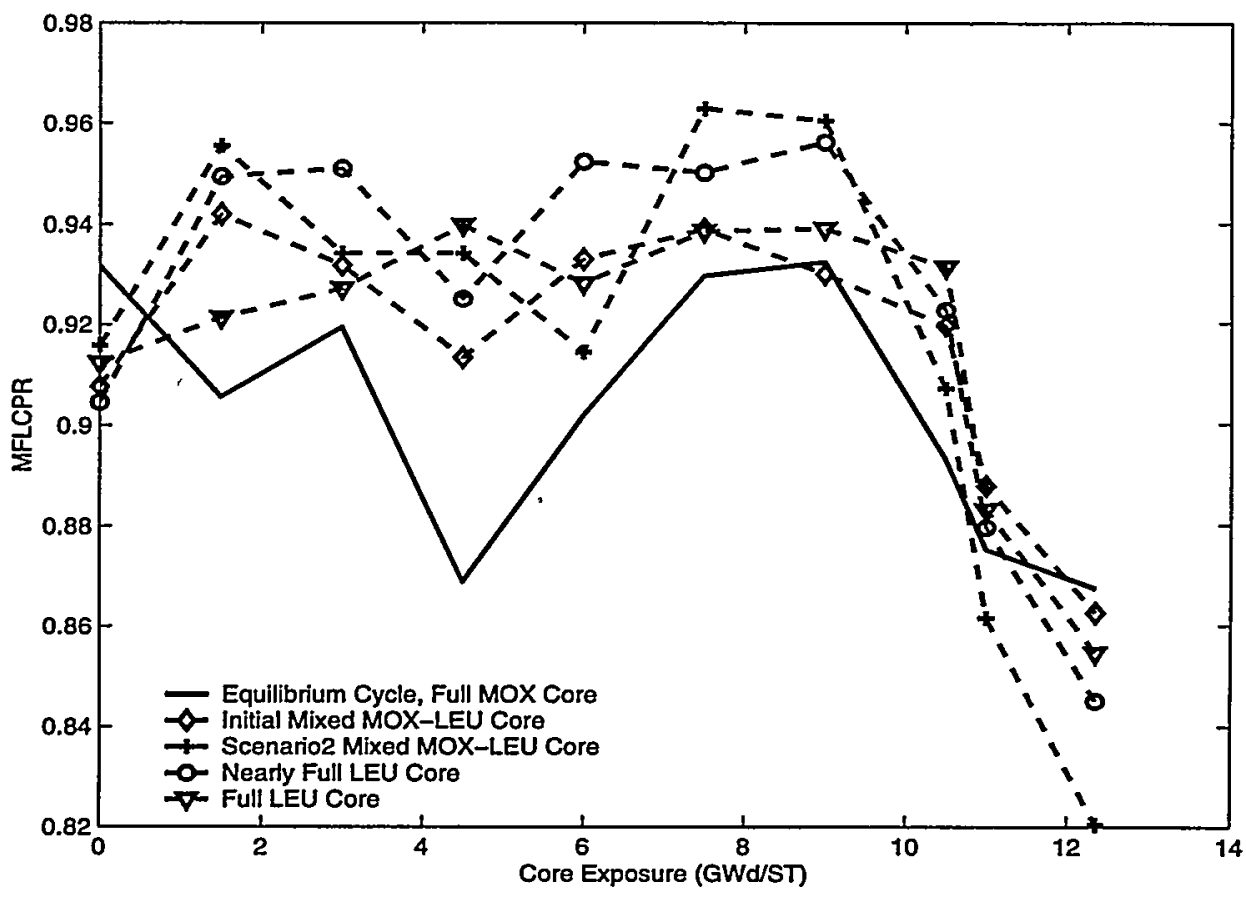

FIGURE 6.30. Summary of MFLCPR for Disruption Scenario 2 Cores

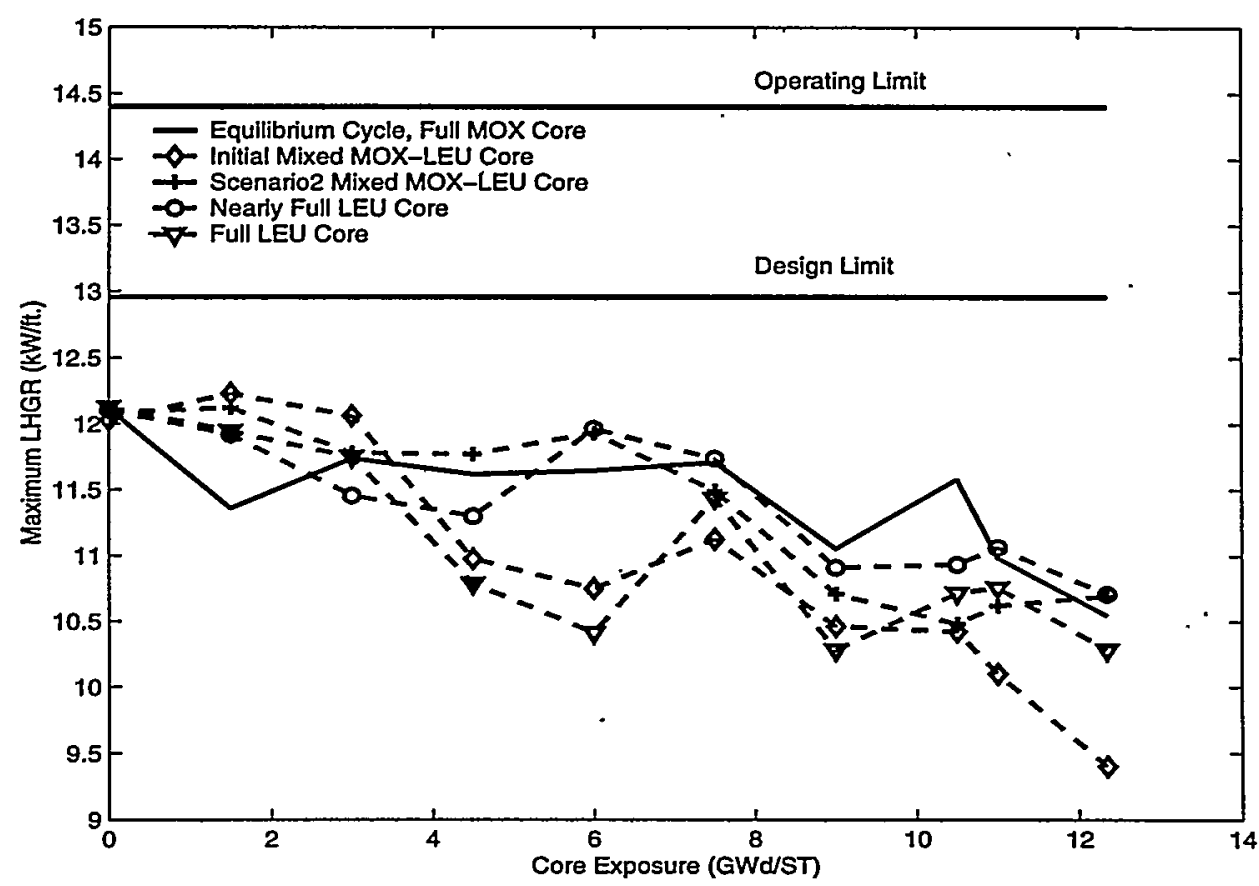

FIGURE 6.31. Summary of Maximum LHGR for Disruption Scenario 2 Cores 


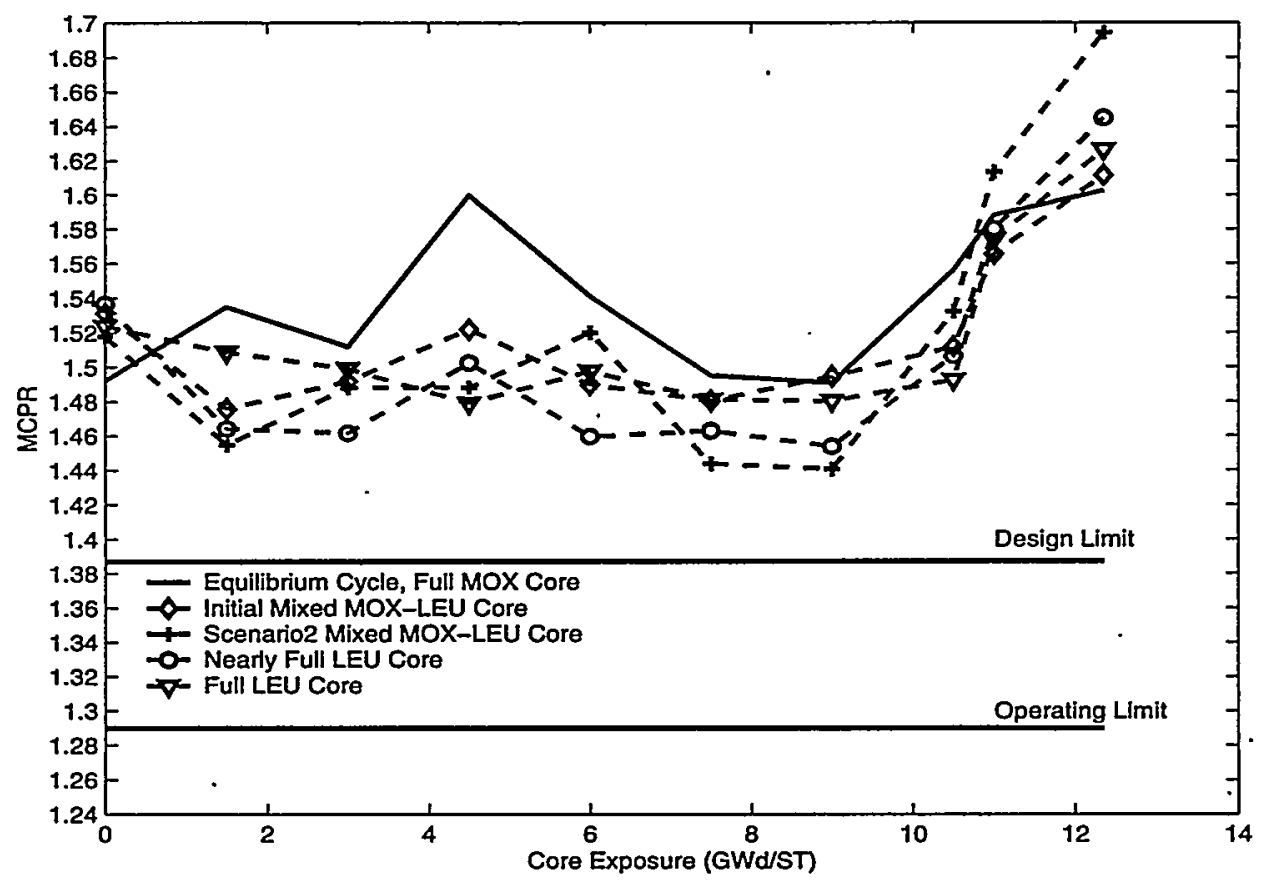

FIGURE 6.32. Summary of MCPR for Disruption Scenario 2 Cores

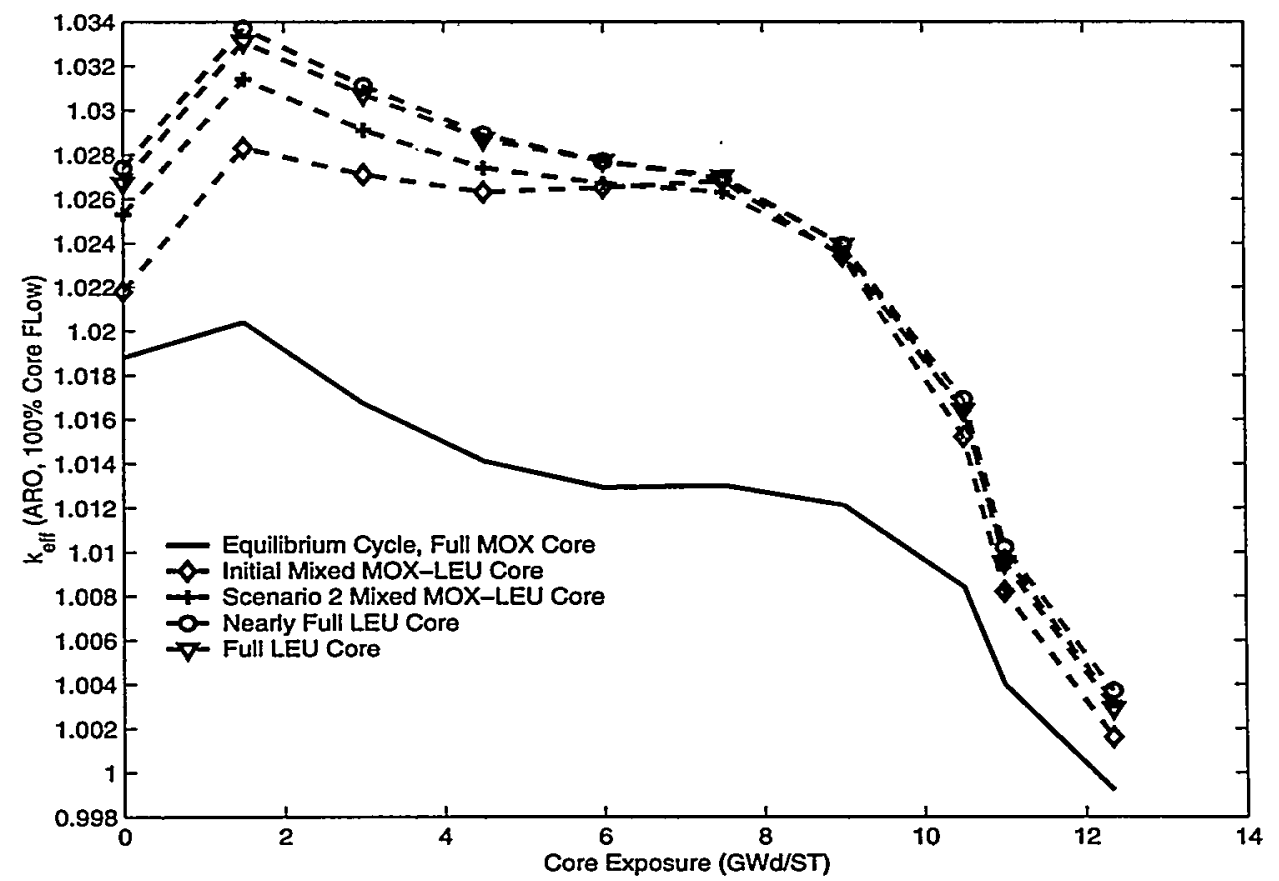

FIGURE 6.33. Summary of $\mathrm{k}_{\mathrm{eff}}$ (ARO, 100\% Core Flow) for Disruption Scenario 2 Cores 


\section{Conclusions}

For the initial mixed LEU-MOX core, starting with the GE equilibrium cycle, full MOX core, an acceptable LP was determined in regard to satisfying all constraints. For the subsequent cycle, the following two disruption scenarios were examined: (1) complete availability of MOX feed assemblies, and (2) complete unavailability of MOX feed assemblies.

The first scenario leads to a full MOX core and the second scenario leads to a full LEU core. Transition cycles from the equilibrium cycle, full MOX core to another full MOX core and from the equilibrium cycle, full MOX core to a full LEU core have been developed. LPs and CRPs found by FORMOSA-B for those transition cycles are judged to be acceptable with regard to the constraints imposed.

\section{Acknowledgment}

The authors would like to thank Trent Primm and Dr. Felix C. Difilippo of Oak Ridge National Laboratory, Dr. Steven Congdon of General Electric, and Dr. Atul Karve of the Electric Power Research Center for their cooperation during the course of this study.

\section{References}

[1] I. ARIANI and P.J. TURINSKY, "Evaluation of Mixed LEU-MOX Loading Patterns for LWRs Originating from Inability to Deliver MOX Assemblies: ABBCE System 80 Reload Core Study," ORNL/SUB/99-19XSY063V-1, Oak Ridge National Laboratory, February 1999. 
[2] I. ARIANI and P.J. TURINSKY, "Evaluation of Mixed LEU-MOX Loading Patterns for LWRs Originating from Inability to Deliver MOX Assemblies: Westinghouse Reload Core Study," ORNL/SUB/99-19XSY063V-2, Oak Ridge National Laboratory, February 1999.

[3] "FMS: The Scanpower Fuel Management System, HELIOS Documentation," Scanpower A/S, 1995.

[4] "FORCIP-B V1.0 Code Manual: FORMOSA-B Cross Section Interface Program," Electric Power Research Center, North Carolina State University, 1998

[5] "FORMOSA-B V1.0 User's Manual: Fuel Management Optimization Software for BWRs," Electric Power Research Center, North Carolina State University, 1999

[6] "Optimization and Implementation Study of Plutonium Disposition using Existing GE Boiling Water Reactors," Contract No. DE-AC01-93SF-19681, GE Nuclear Energy, September 1996. 


\section{Appendices}

HELIOS, ZENITH, FORCIP-B and FORMOSA-B input and output files for cores examined in this study are available in the /u10/fmdp2/GEBWR.DIR directory. Below is the summary of the contents of the /u10/fmdp2/GEBWR.DIR directory:

HELIOSINPUT . DIR

MOX Lattice:

Lower:

1. lower0.inp

: 0\% Void, Unrodded

2. Iower 40 .inp

: 40\% Void, Unrodded

3. Iower 70 : inp

: $70 \%$ Void, Unrodded

Middle:

4.lower40CRH.inp : $40 \%$ Void, Rodded

. ' Upper:

5.middle0.inp : $0 \%$ Void, Unrodded

6.middle40.inp : $40 \%$ Void, Unrodded

7.middle70.inp $\quad: 70 \%$ Void, Unrodded

8.middle40CRH.inp : $40 \%$ Void, Rodded

9. upper0.inp

10. upper 40 .inp

11.upper70.inp

: 0\% Void, Unrodded

12 . upper $40 \mathrm{CRH}$. inp

: 40\% Void, Unrodded

: 70\% Void, Unrodded

: 40\% Void, Rodded

LEU Enrichment 2 Lattice:

Lower:

13.1leulower00.inp : 0\% Void, Unrodded

14.1leulower40.inp : 40\% Void, Unrodded

15.1leulower70.inp : 70\% Void, Unrodded Middle:

16.1leulower40CRH.inp: $40 \%$ Void, Rodded

17. Ileumiddle0.inp : $0 \%$ Void, Unrodded

18. Ileumiddle40.inp : 40\% Void, Unrodded

19.1leumiddle70.inp : 70\% Void, Unrodded

20.Ileumiddle $40 \mathrm{CRH}$.inp: $40 \%$ Void, Rodded Upper :

21.1leuupper00.inp : 0\% Void, Unrodded

22.1leuupper40.inp : 40\% Void, Unrodded

23.1leuupper70.inp : 70\% Void, Unrodded

24.1leuupper40CRH.inp: 40\% Void, Rodded 
LEU Enrichment 3 Lattice:

Lower:

25.hleulower00.inp : 0\% Void, Unrodded

26.hleulower40.inp : 40\% Void, Unrodded

27.hleulower70.inp : 70\% Void, Unrodded

28.hleulower40CRH.inp: $40 \%$ Void, Rodded Midale:

29.hleumiddle0.inp : 0\% Void, Unrodded

30.hleumiddle40.inp : 40\% Void, Unrodded

31.hleumiddle70.inp : 70\% Void, Unrodded

32.hleumiddle40CRH.inp: $40 \%$ Void, Rodded Upper :

33.hleuupper00.inp : 0\% Void, Unrodded

34.hleuupper40.inp : 40\% Void, Unrodded

35.hleuupper70.inp : 70\% Void, Unrodded

36.hleuupper $40 \mathrm{CRH}$. inp: $40 \%$ Void, Rodded

ZENITHINPUT.DIR

File names are in a similar format to the HELIOS input file names. Add ' $z$ ' at the beginning of each file name. Example: zlower0.inp is the ZENITH input file for MOX lattice (lower, o\% void, unrodded).

Number of files $=36(37-72)$

ZENITHOUTPUT.DIR

File names follow the format of filenames for ZENITH input. Replace ".inp" with ".out". Example: zlower0.out is the ZENITH output file for MOX lattice (lower, 0\% void, unrodded) .

Number of files $=36(72-108)$

Cross sections for reflector regions:

109. bwrrref.cax

110. rrefcld.cax

111. bwrbref.cax

112. brefcld.cax

113. bwrtref0.cax

114. bwrtref4.cax

115. bwrtref7. cax

116. trefcld.cax 
FORCIPB.DIR

117. forcip.inp : FORCIP-B input file

118. forcipb.table : FORCIP-B output file

119. fcpv1.0.sun. : FORCIP-B executable (SUN)

FORMOSAB.DIR

120. fsbv1.5.sun : FORMOSA-B executable (SUN)

121. basic.data : Basic Operational Data

122. steam.data :

123 spcvmox.dat :

Files in the following subdirectories have the following format:

form.cntl : FORMOSA-B Control File

* inp $\quad$ FORMOSA-B Input File

*. cYc : FORMOSA-B Cycle Data File

*out : FORMOSA-B Output File

*aro.inp : FORMOSA-B Input File (ARO, 100\% Core Flow)

*aroout : FORMOSA-B Output File (ARO, 100\% Core Flow)

EQCYCFULLMOX.DIR : Equilibrium Cycle Full MOX Core

124. form.cntl

125. eqcycfullmox.inp

126. eqcycfullmox.cyc

127. eqcycfullmoxout

128. eqcycfullmoxaro.inp

129. eqcycfullmoxaroout

INITMIXED.DIR : Initial Mixed MOX-LEU Core

130. form.cntl

131. initmixmoxleu.inp

132. initmixmoxleu.cyc

133. initmixmoxleuout

134. initmixmoxleuaro.inp

135. initmixmoxleuaroout

SCENARIO1.DIR : Disruption Scenario1 Cores

136. form.cntl

137. slmix.inp : Scenario 1 Mixed MOX-LEU Core

138. slmix.cyc 
139. simixout

140. slmixaro.inp

141. slmixaroout

142. SIbmix.inp: Scenario 1b Mixed MOX-LEU Core

143. slbmix. cyc

144. slbmixaro.inp

145. slbmixaroout

146. optslbmix.inp: Optimized LP / CRP

147. opts1bmixout

148. opts1bmixaro.inp

149. opts1bmixaroout

150. nearlymox.inp

151. nearlymox.cyc

152. nearlymoxout

153. nearlymoxaro.inp

154. nearlymoxaroout

155. fulimox.inp

156. fullmox.cyc

157. fullmoxout

158. fullmoxaro.inp

159. fullmoxaroout

\section{SCENARIO2.DIR}

: Disruption Scenario2 Cores

136. form.cntl

137. s2mix.inp : Scenario 2 Mixed MOX-LEU Core

138. s2mix.cyc

139. s2mixout

140. s2mixaro.inp

141. s2mixaroout

146. opts2mix.inp: Optimized LP / CRP

147. opts 2 mixout

148. opts2mixaro.inp

149. opts2mixaroout

150. nearlyleu.inp

151. nearlyleu.cyc

152. nearlyleuout

153. nearlyleuaro.inp

154. nearlyleuaroout

155. fullleu.inp

156. fullieu.cyc

157. fullleuout

158. fullleuaro.inp

159. fullleuaroout 


\section{A1. HELIOS Sample Input File}

HELIOS input files for MOX lattices are available in the subdirectories of $/ \mathrm{u} 10 / \mathrm{fcd} /$ helios_user_dir/XSBWR. The following is a HELIOS input file for one of the LEU lattice designs employed in the mixed MOX-LEU cores. The complete HELIOS/ZENITH input files for LEU lattices are available in /u10/fmdp2/GEBWR.DIR.

\section{HELIOS Input File for Lower LEU Enrichment 2 Lattice}

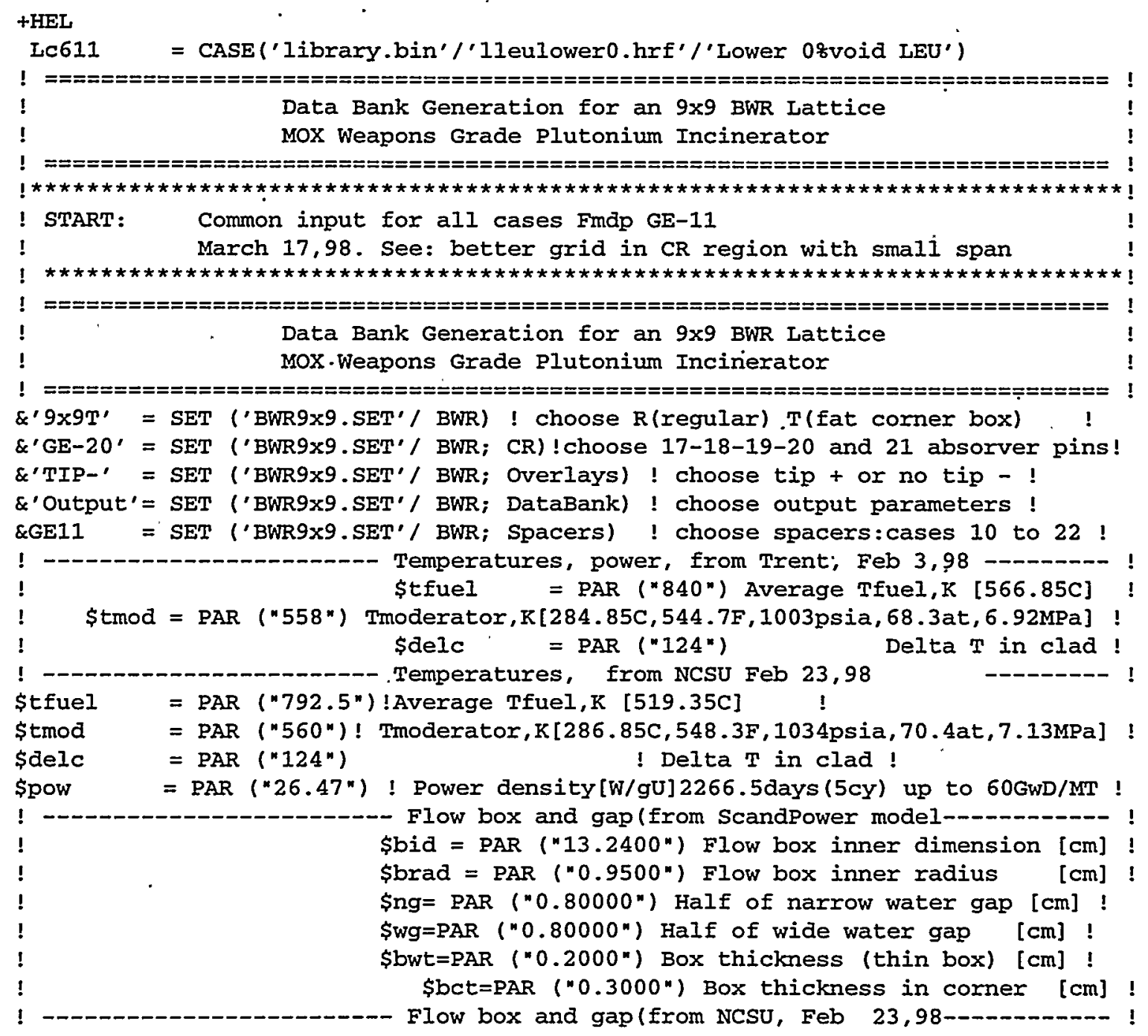




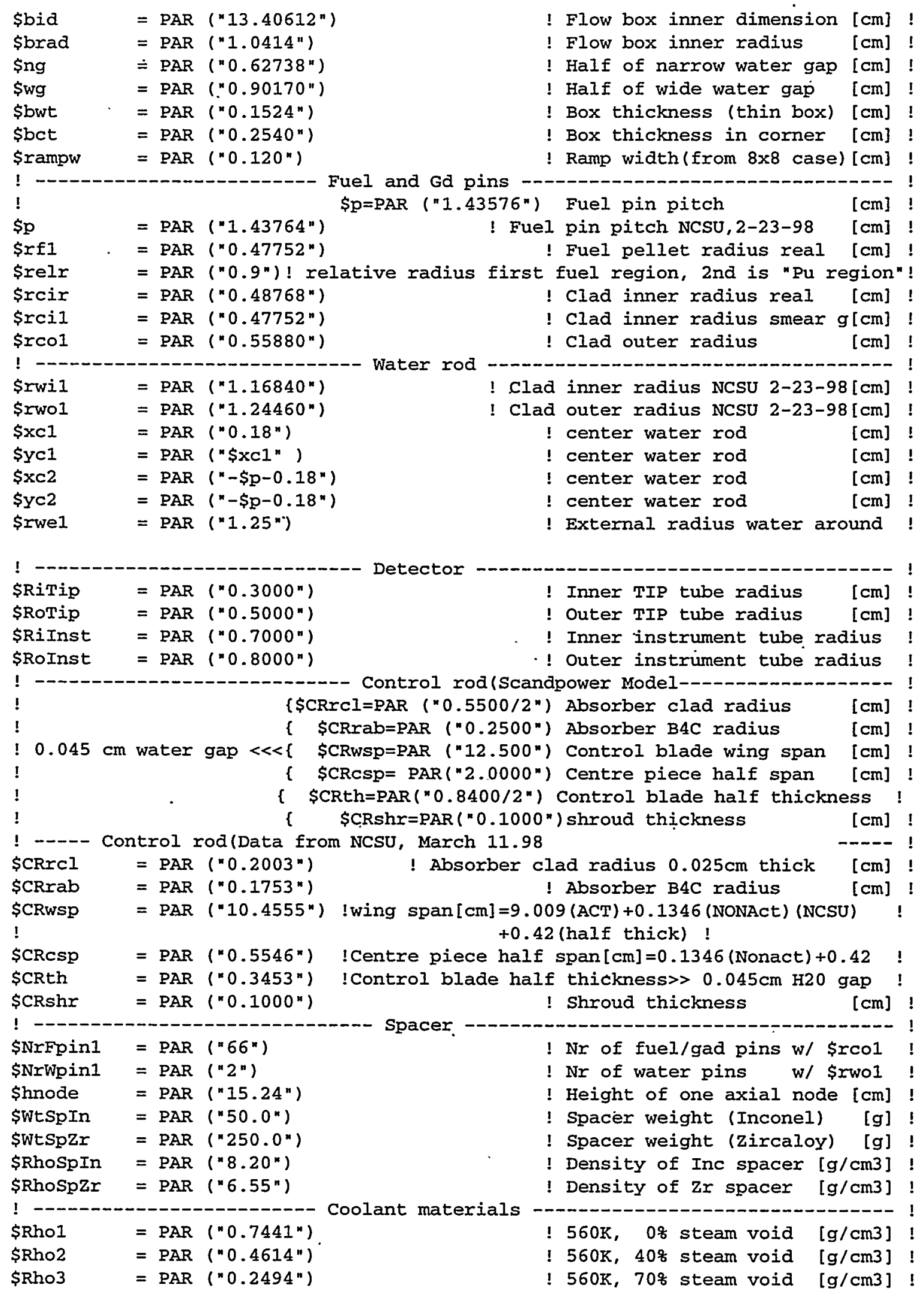




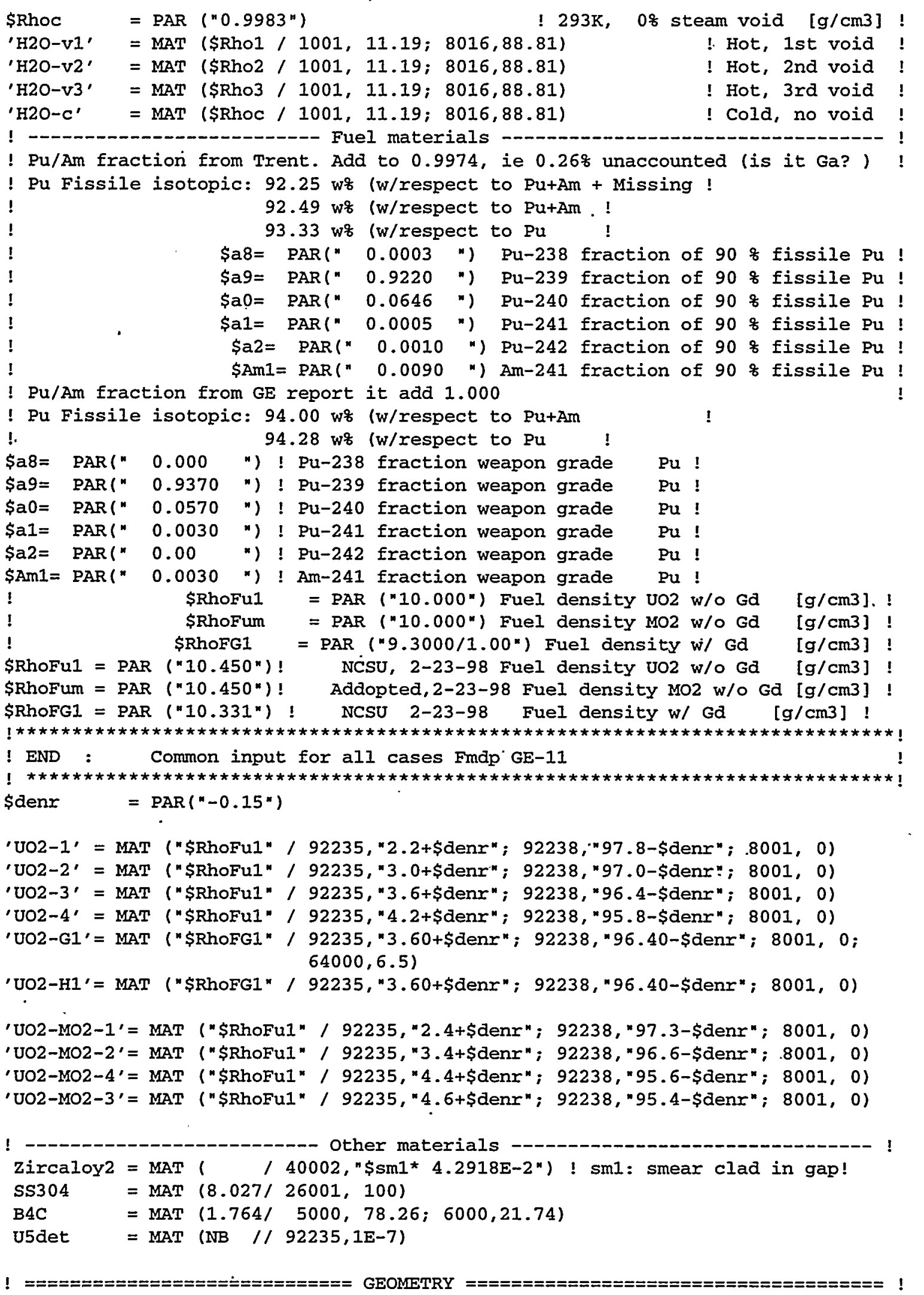




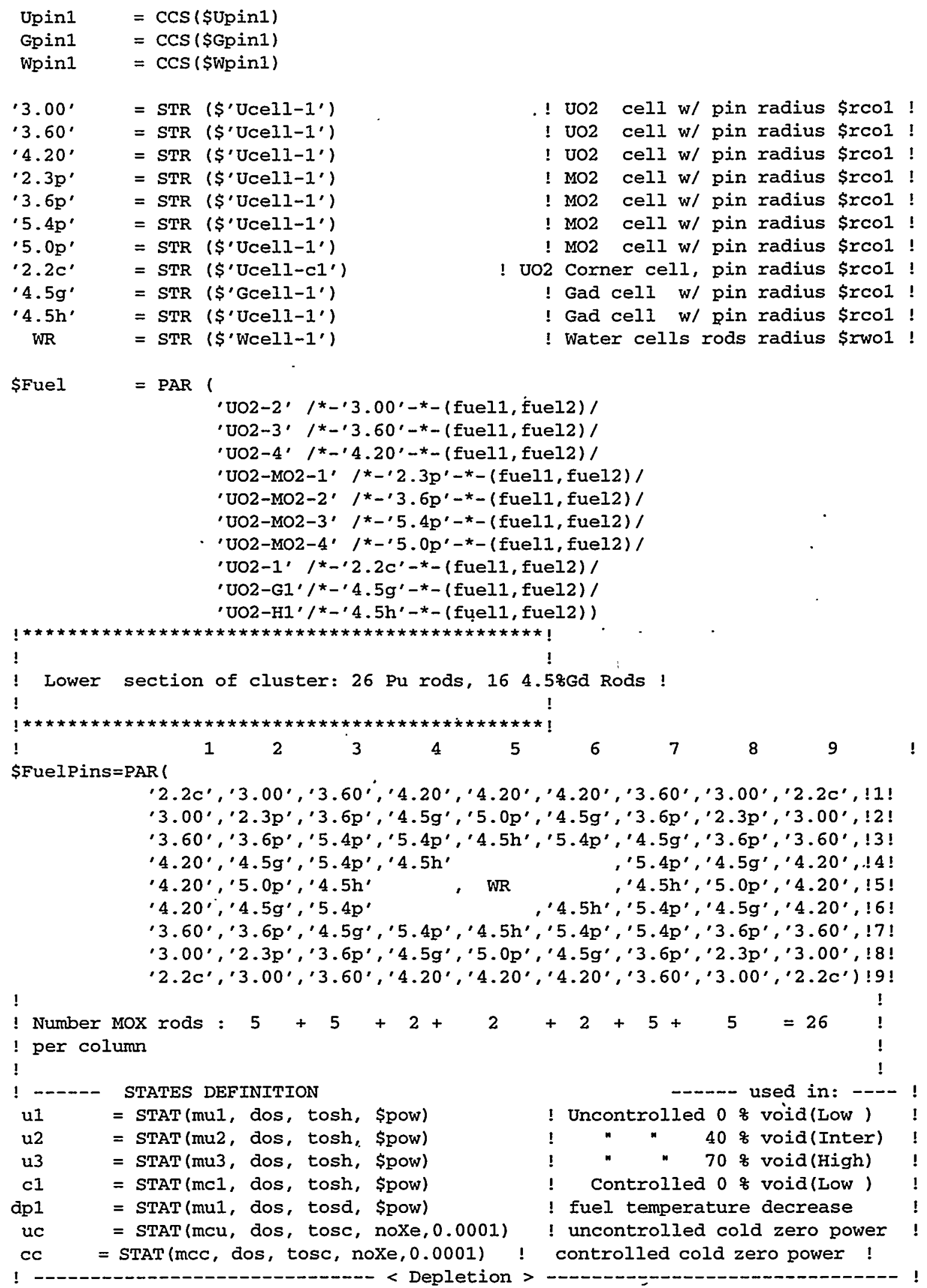




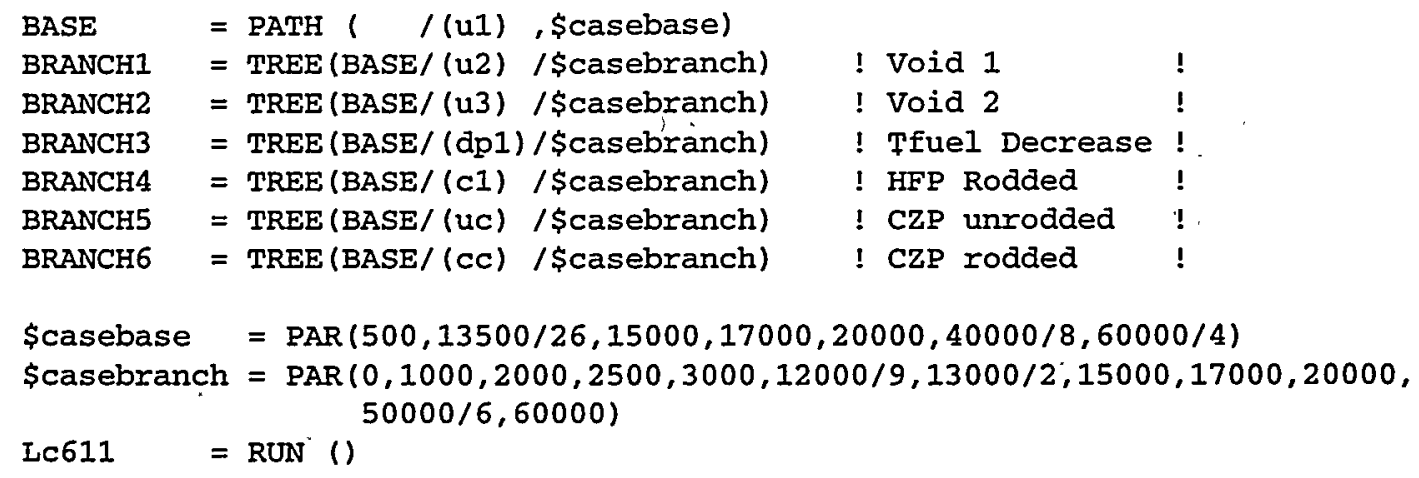

\section{A2. ZENITH Sample Input/Output File}

The following is a ZENITH input/output (input file is contained in the output file) file for the lower axial segment of the LEU enrichment 2 lattice.

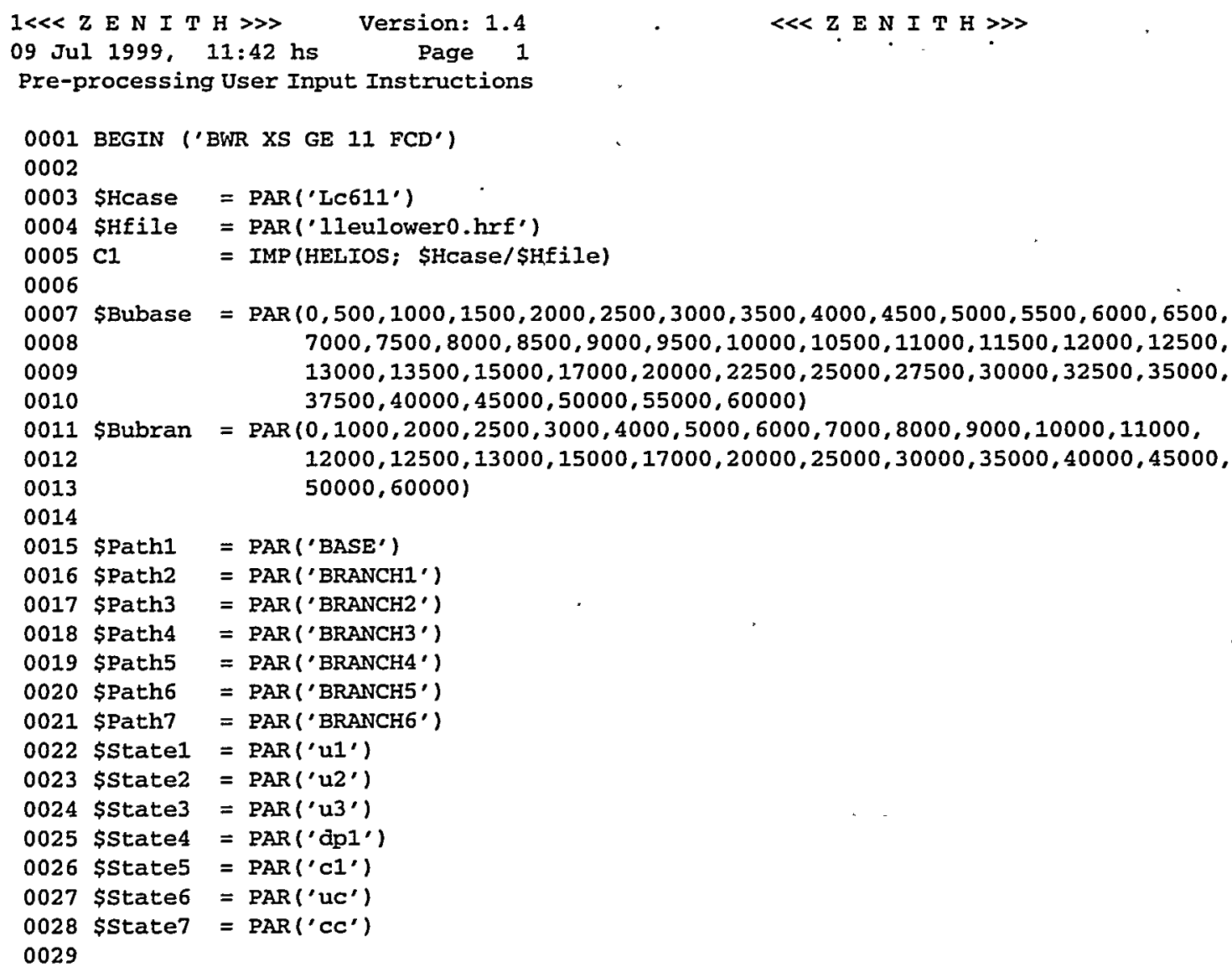




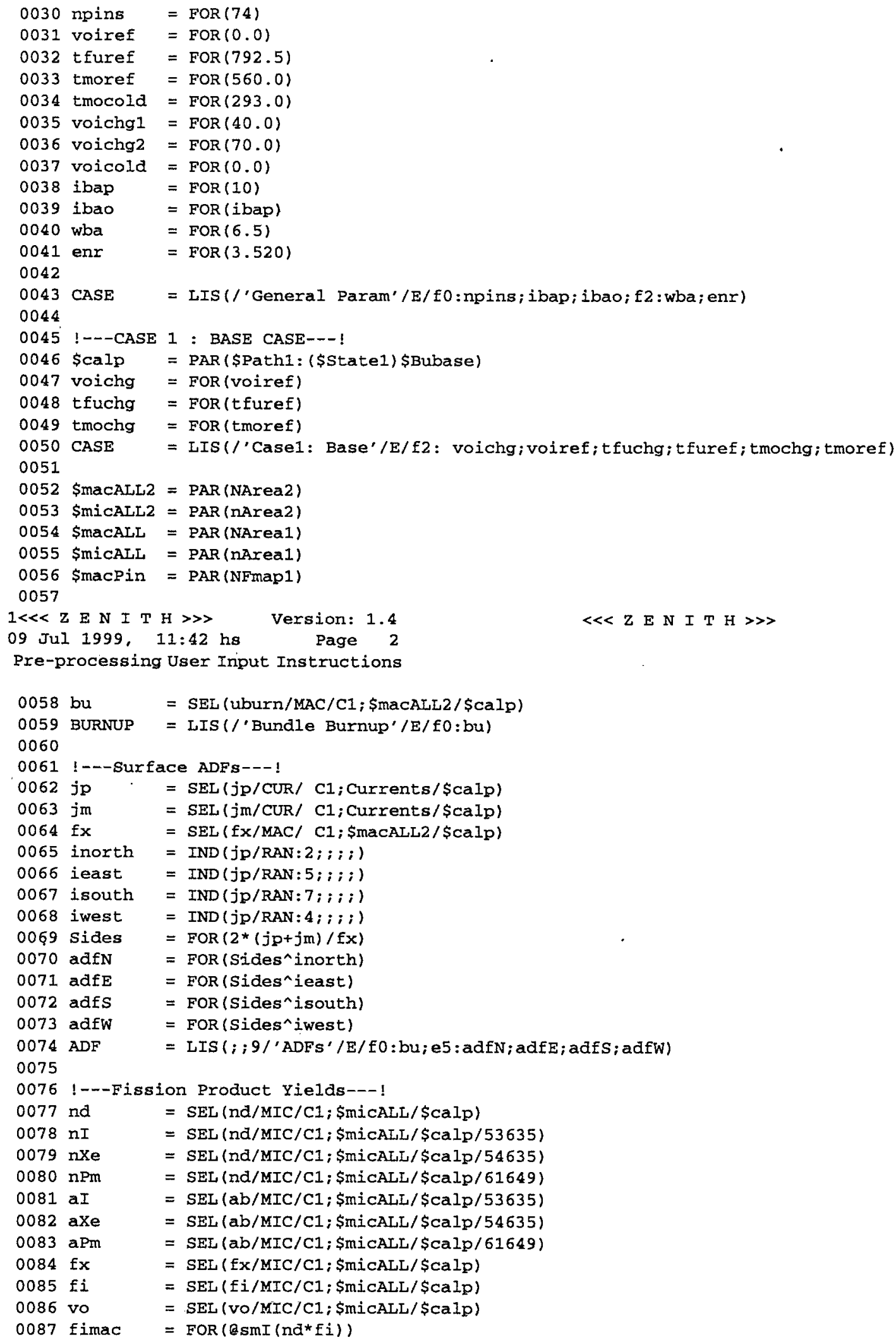




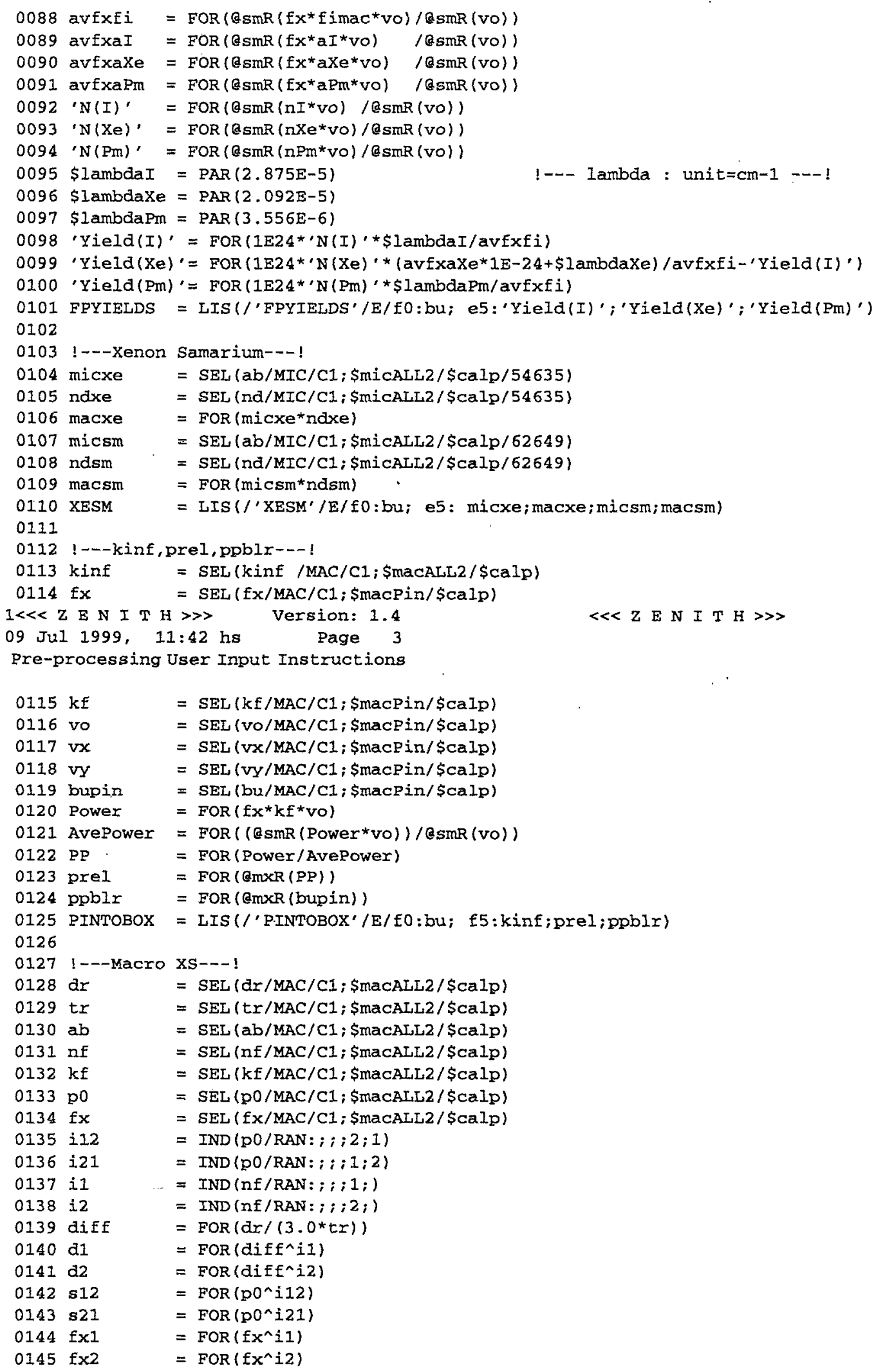




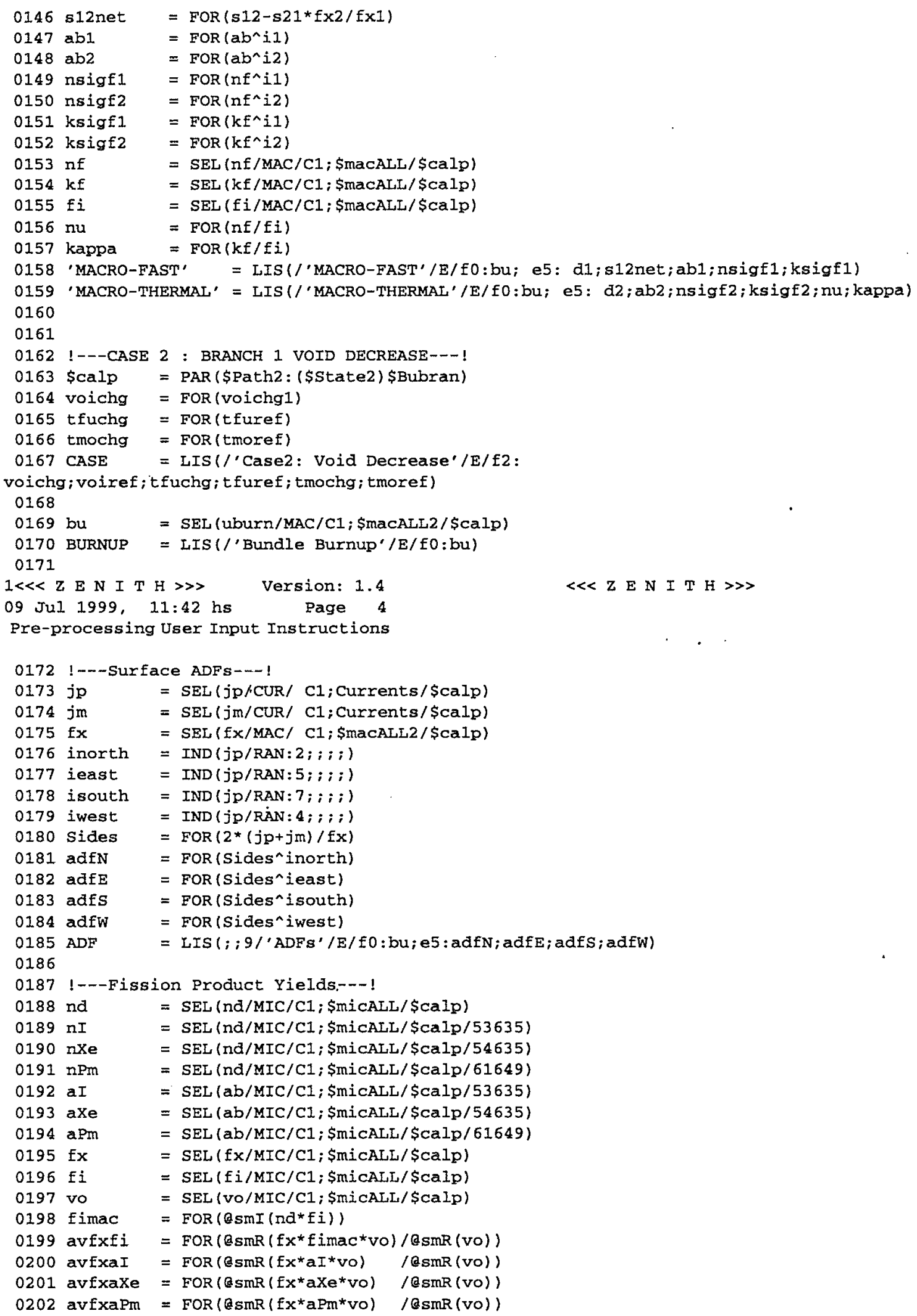




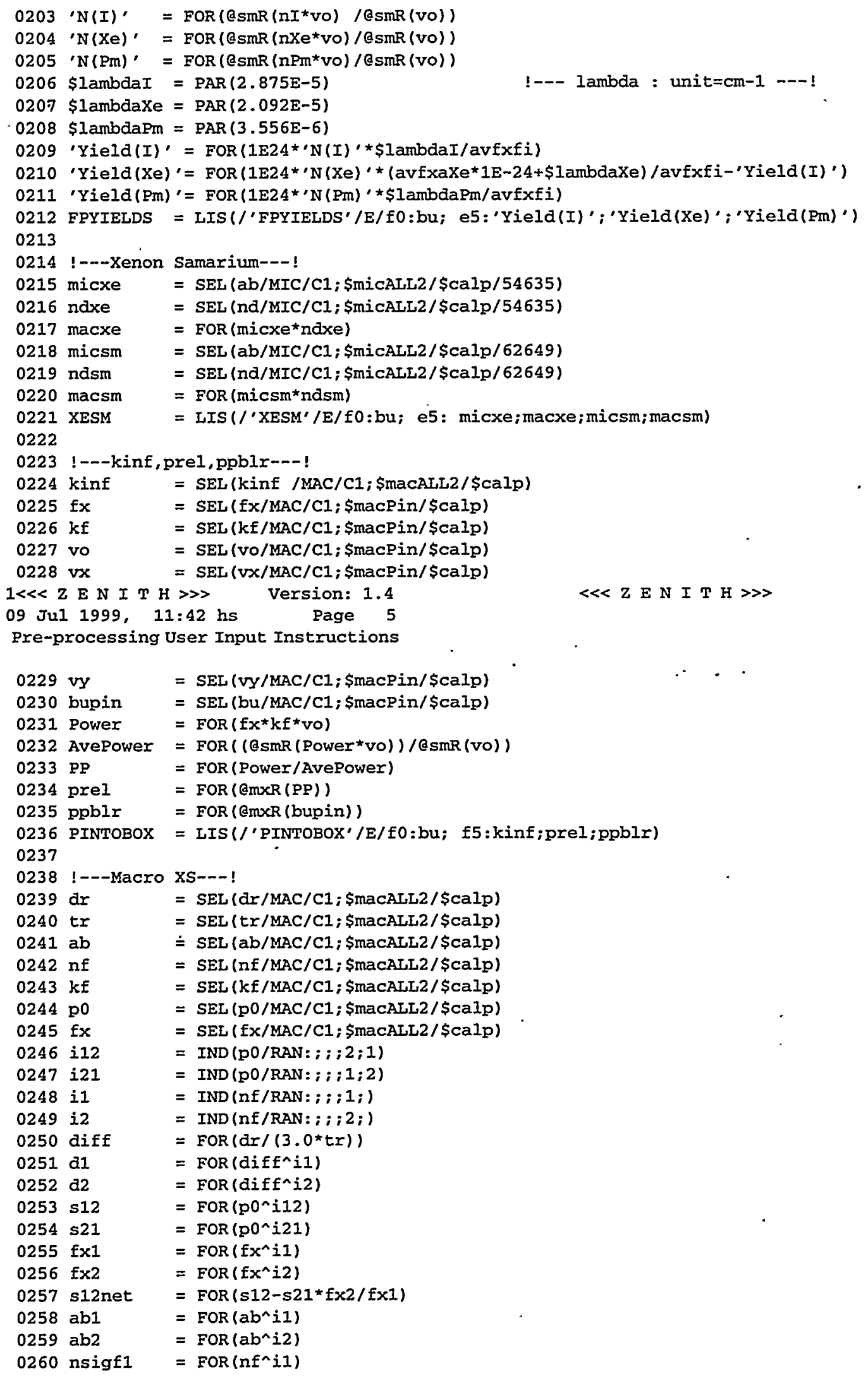




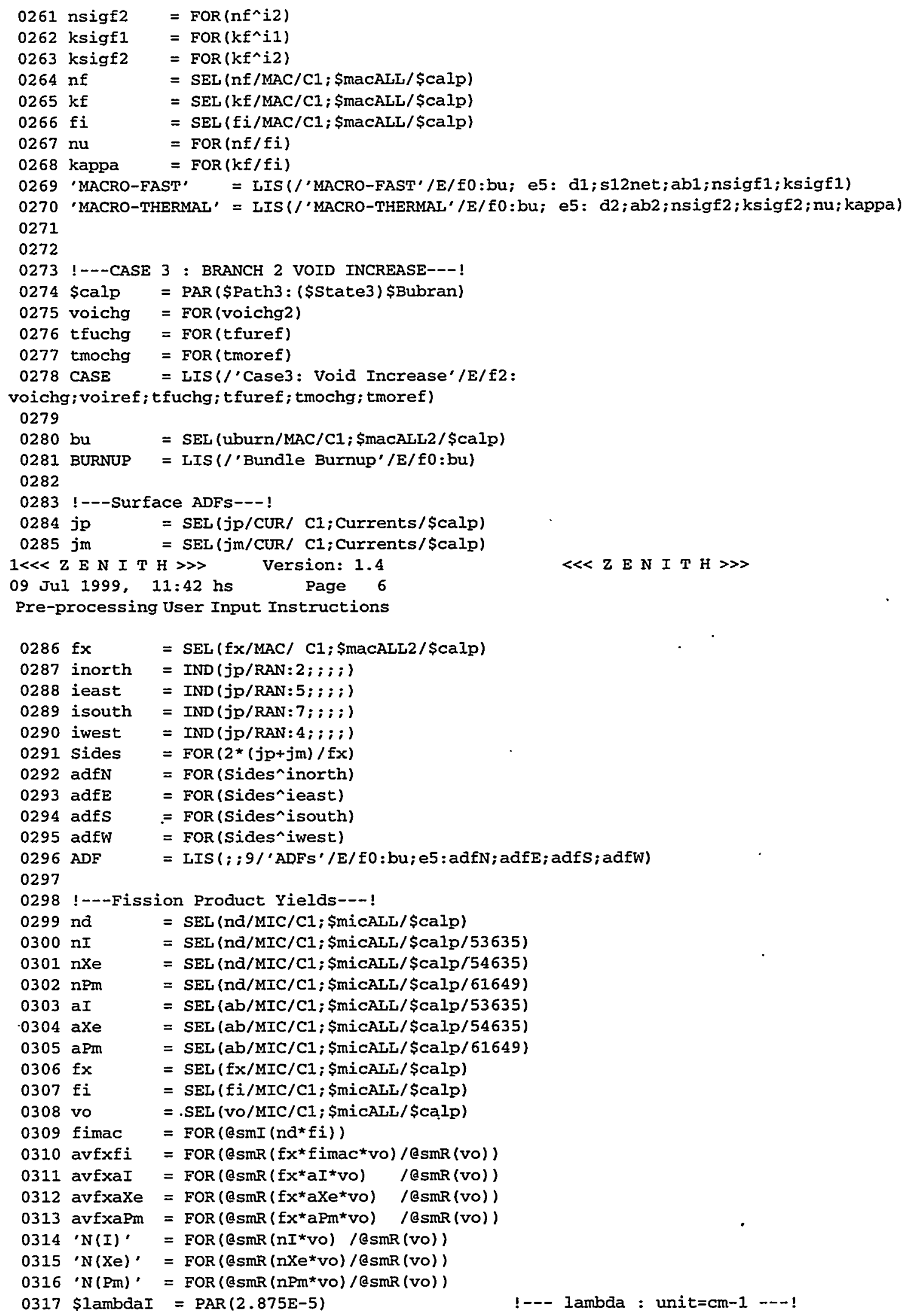




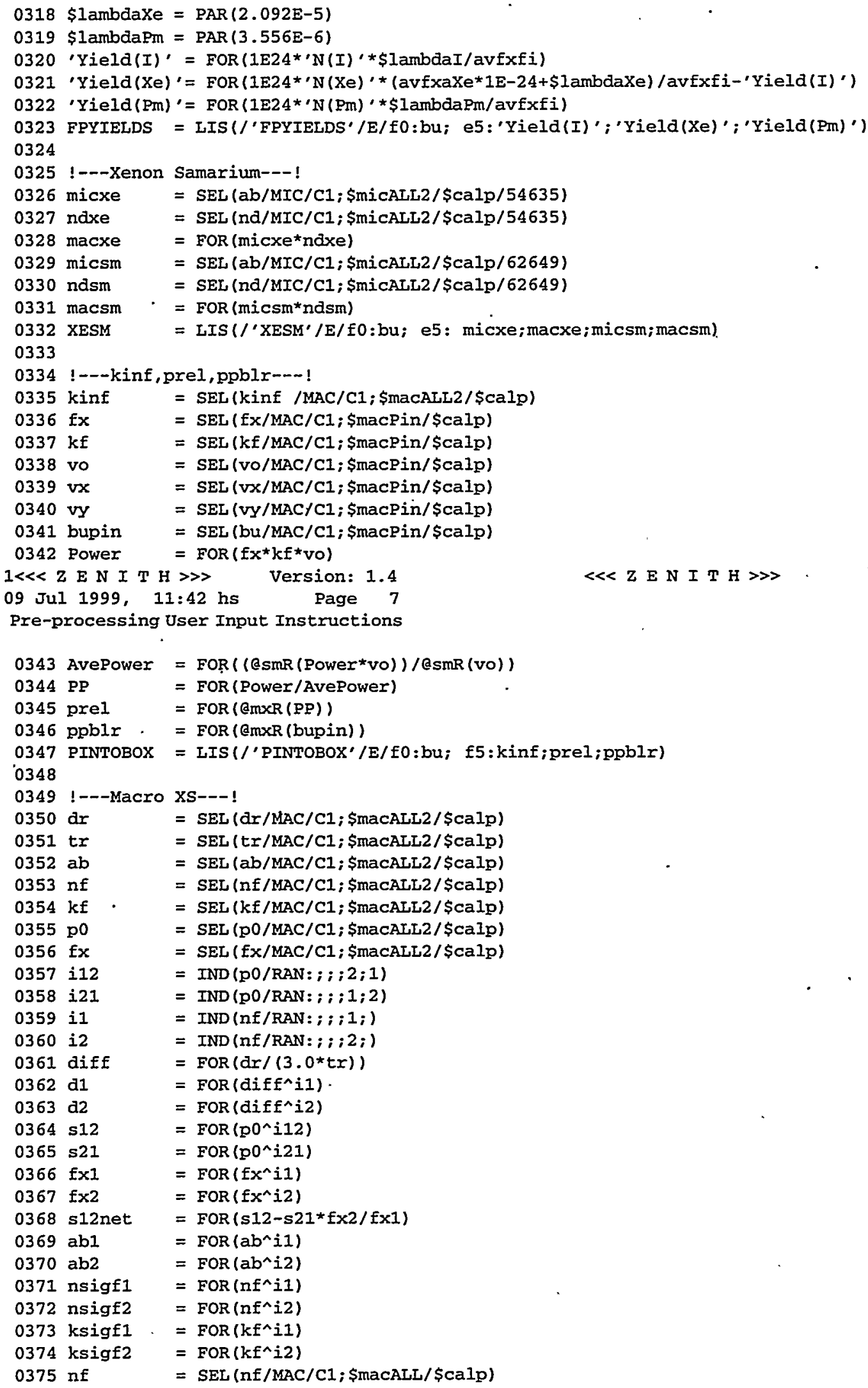




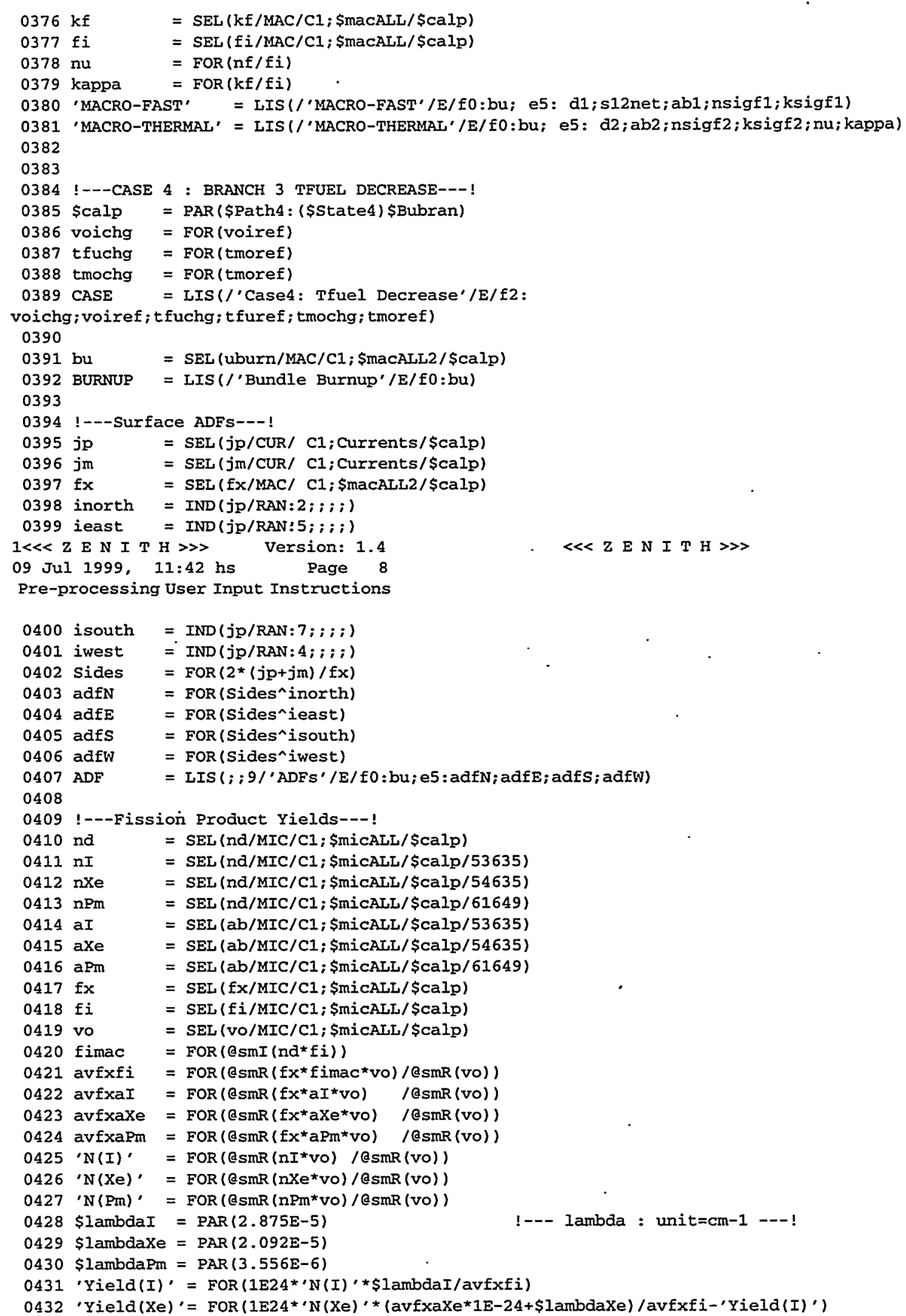




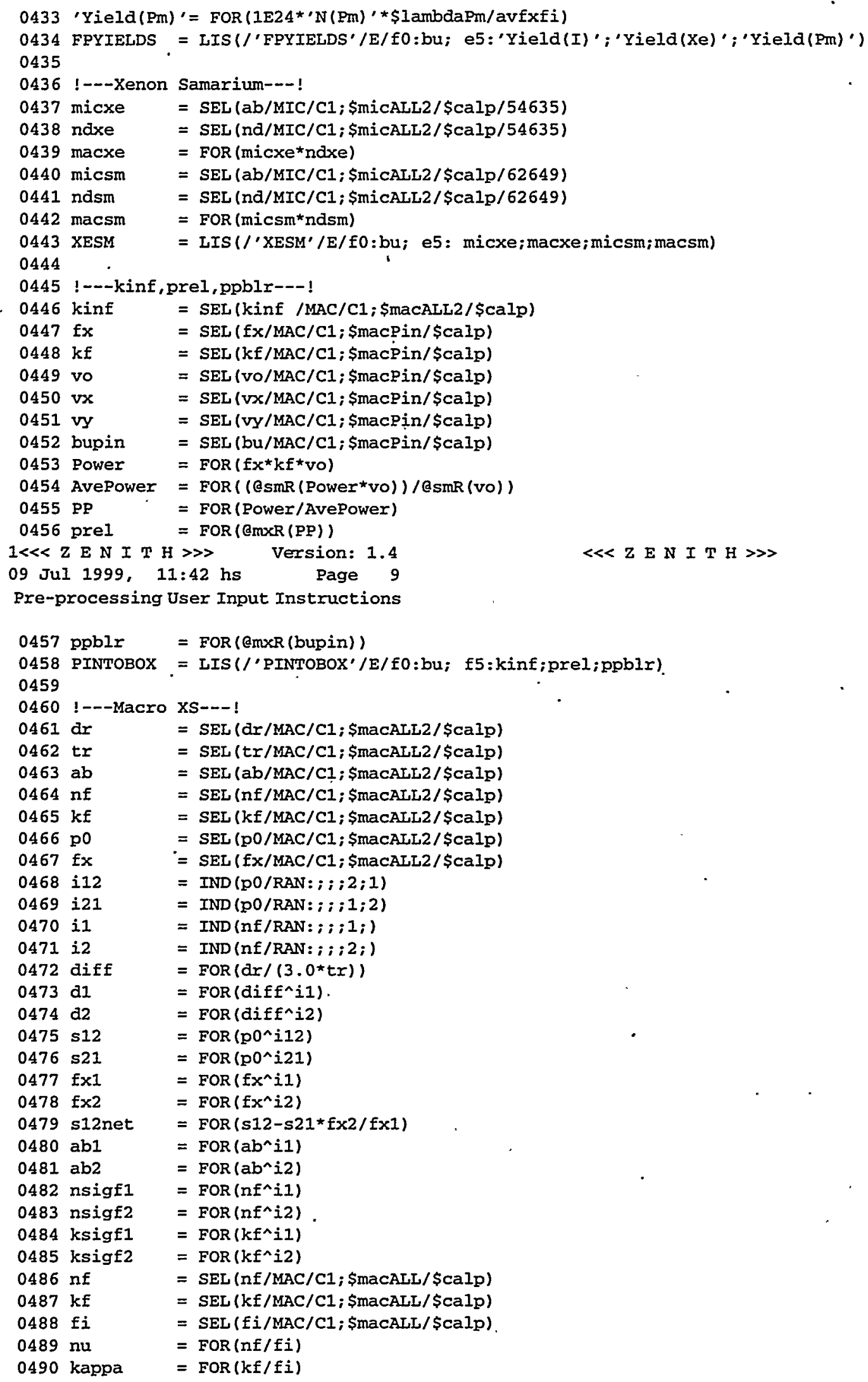




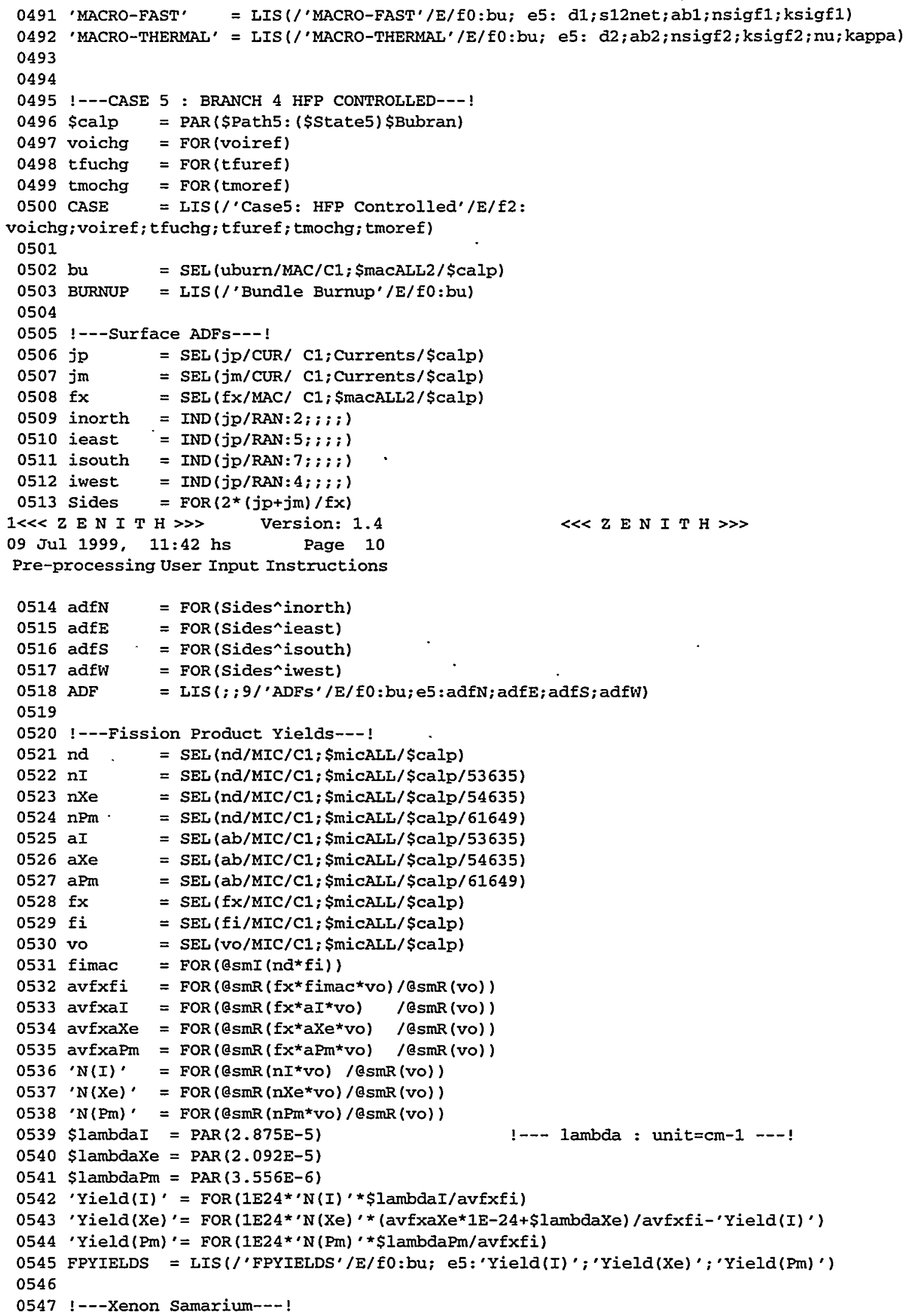


$\checkmark$

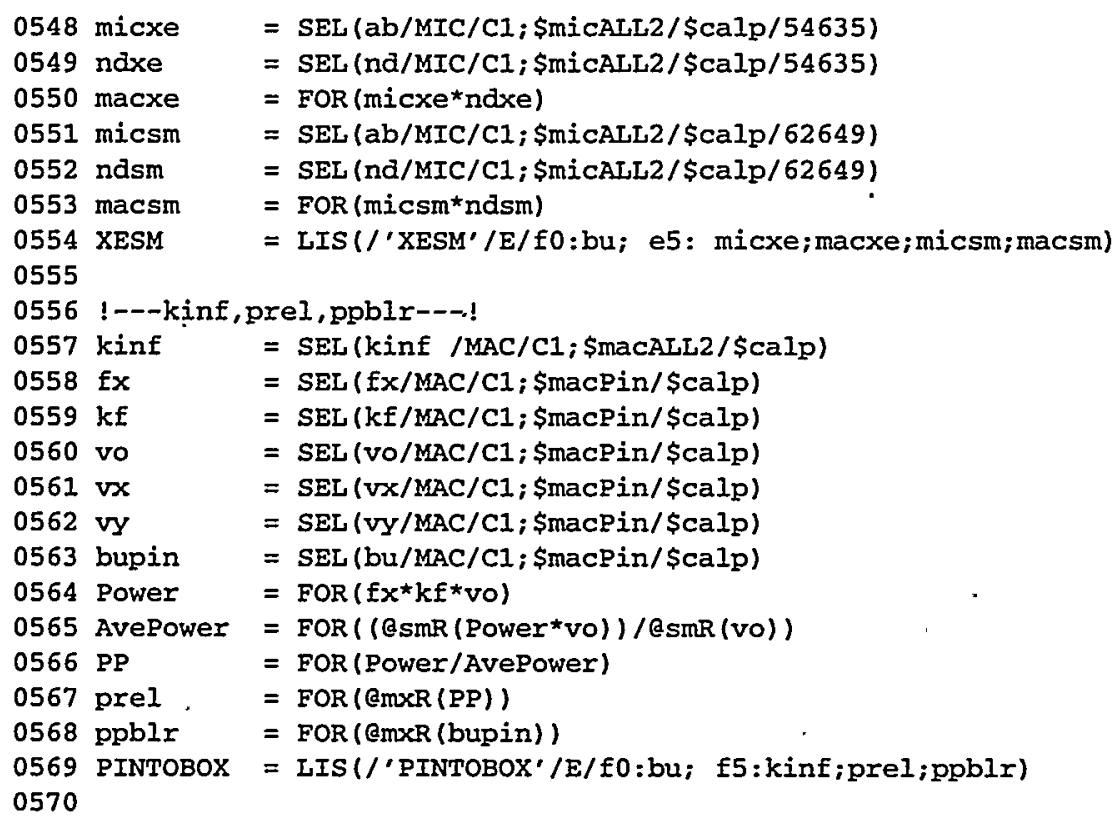




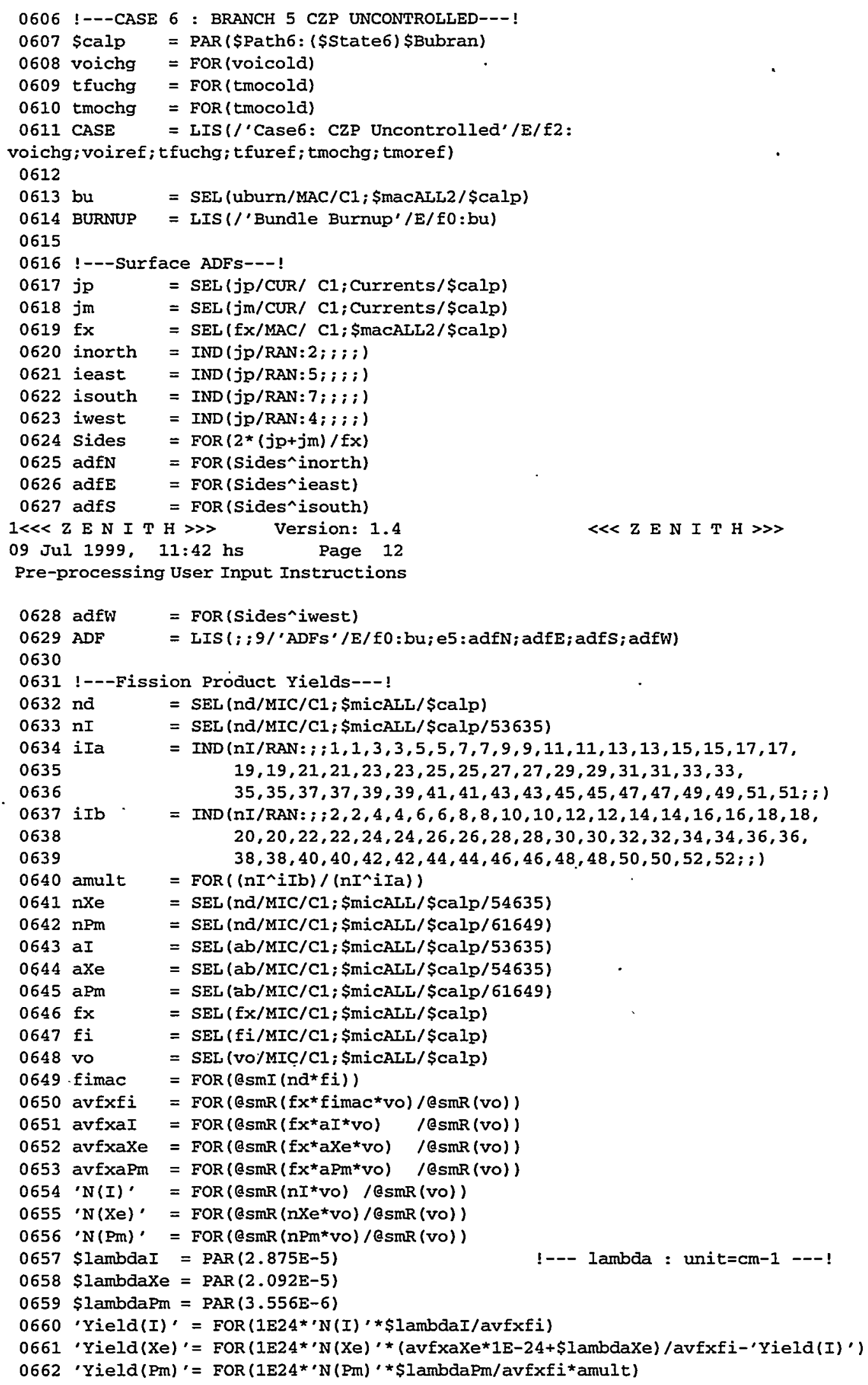




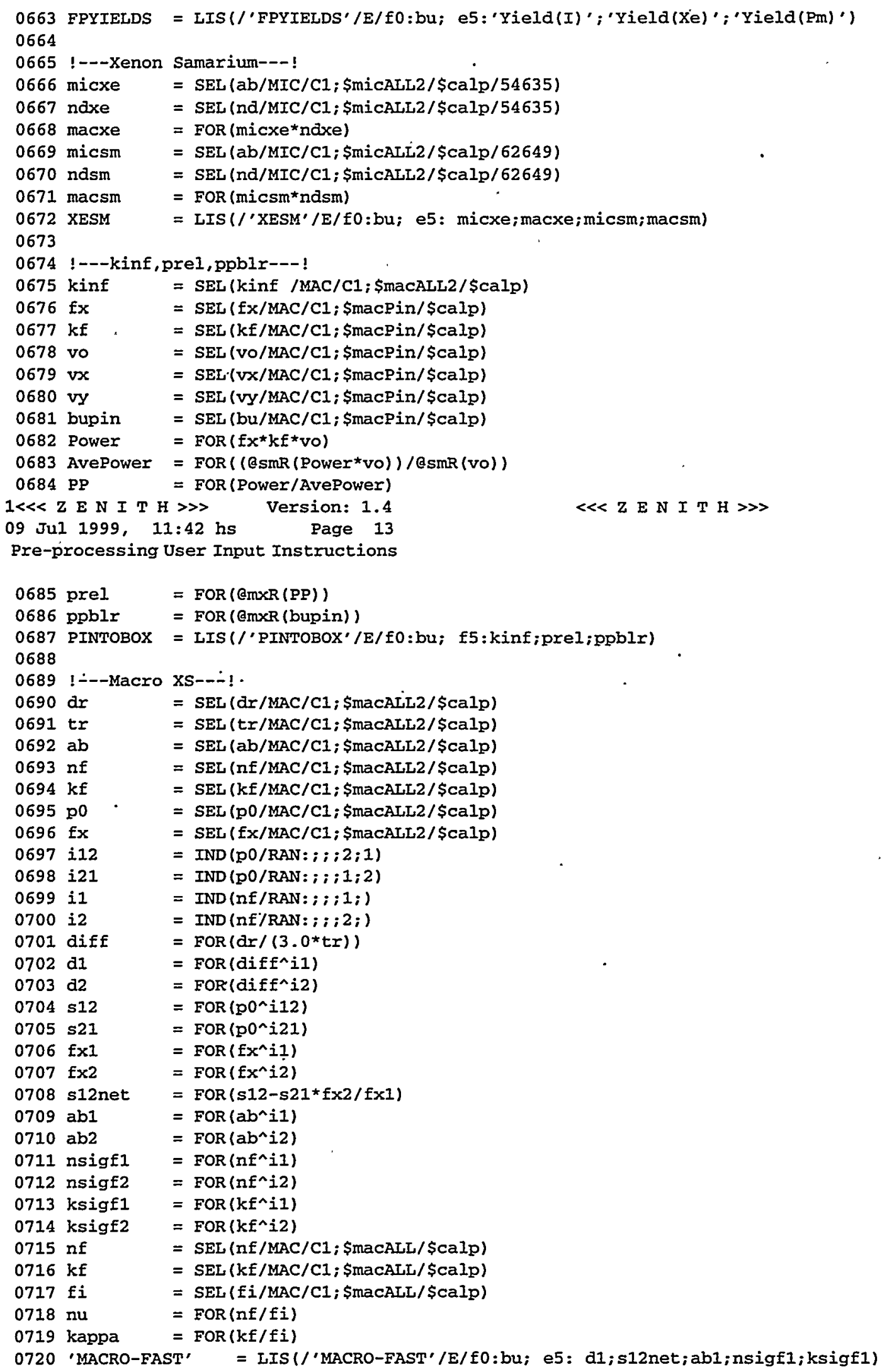




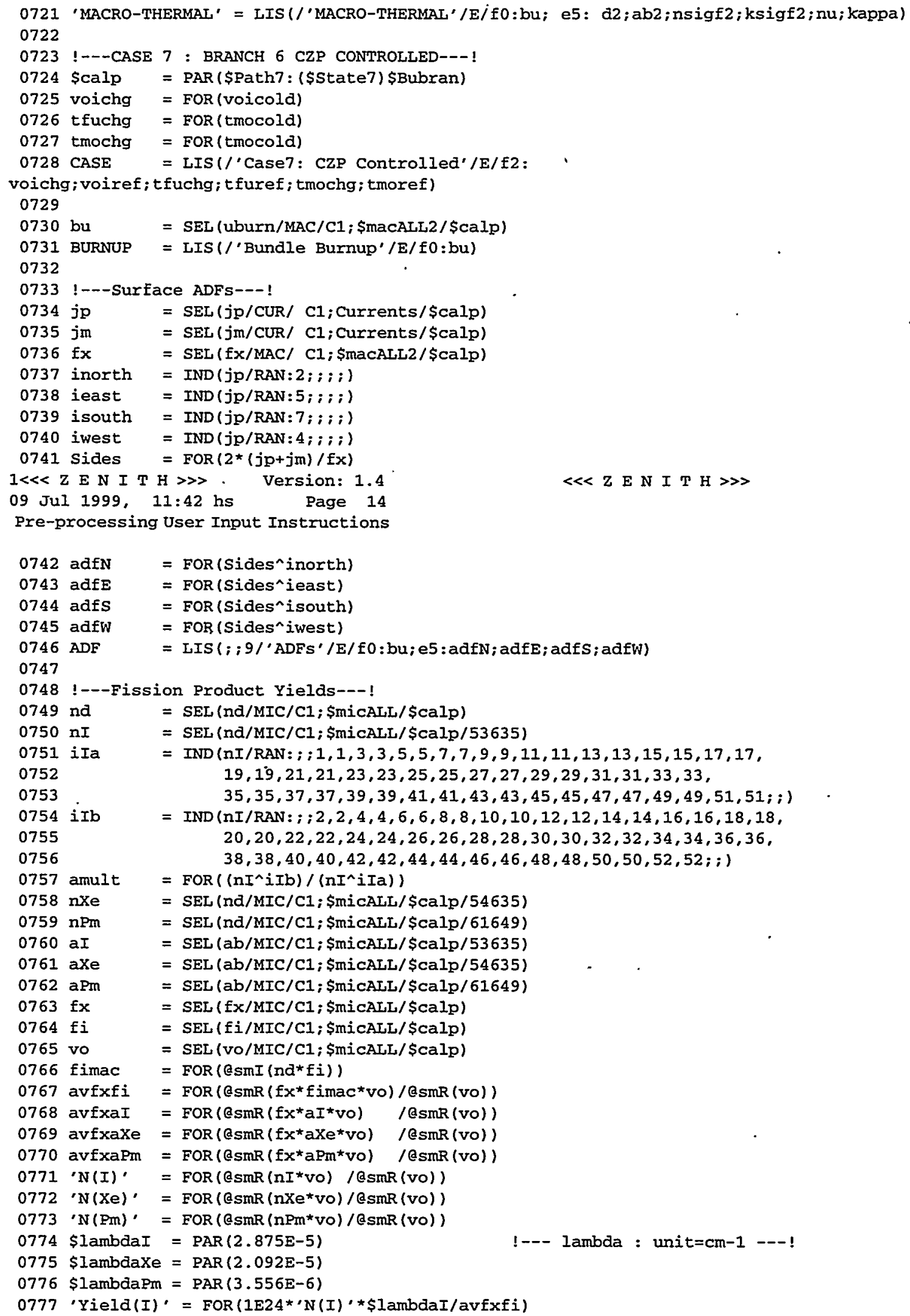




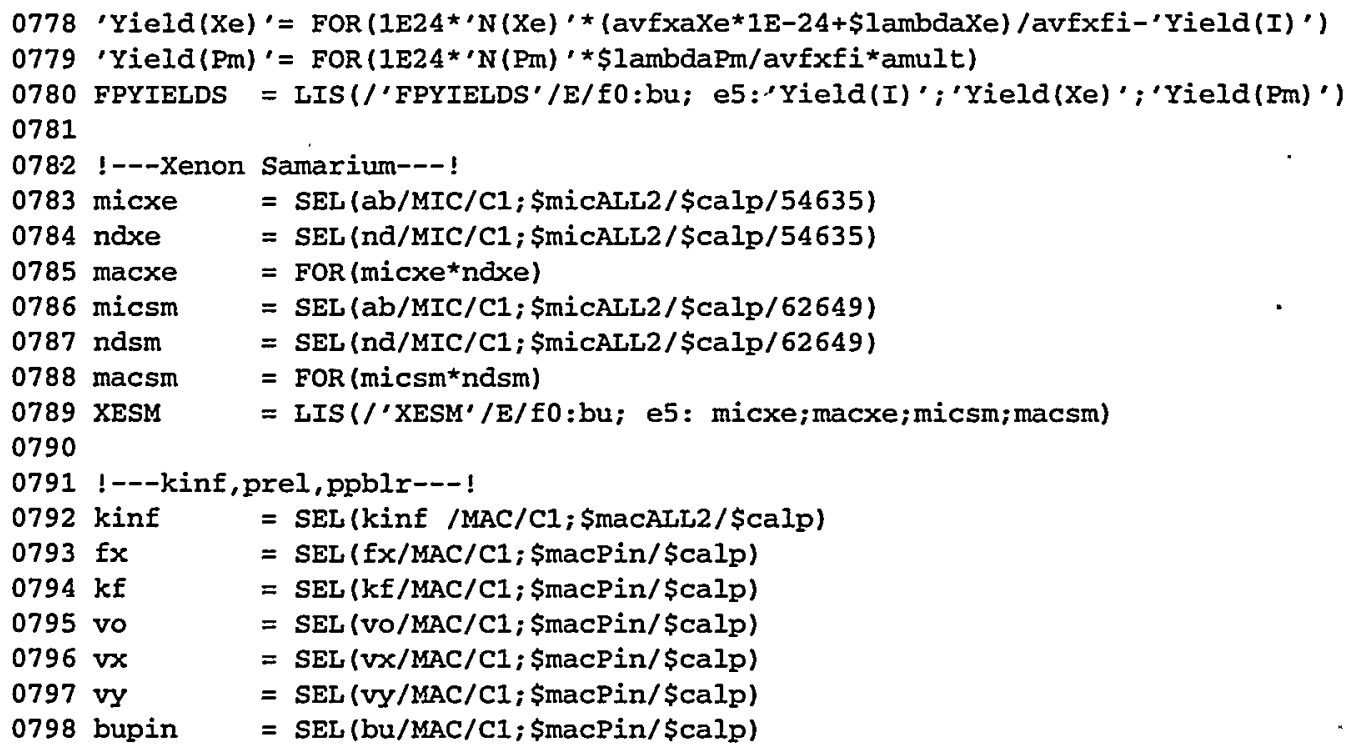




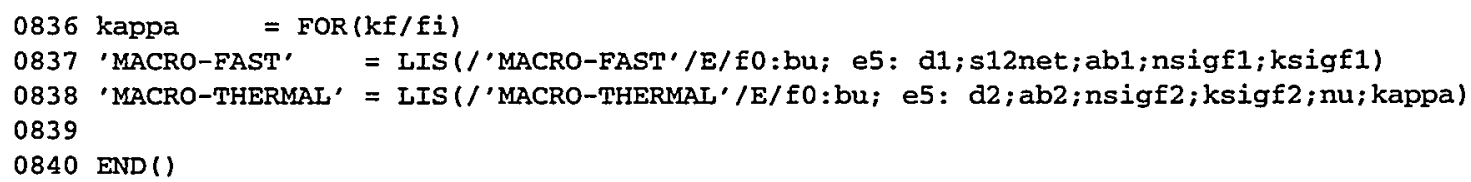

\section{A3. FORCIP-B Input File}

FORCIP-B is an interface code which reads and processes cross sections from the lattice physics code's outputs, the output of FORCIP-B utilized as input to the FORMOSA-B code. The FORCIP-B code has been modified to read HELIOS/ZENITH outputs. For the complete documentation on the FORCIP-B input file, please refer to [4].

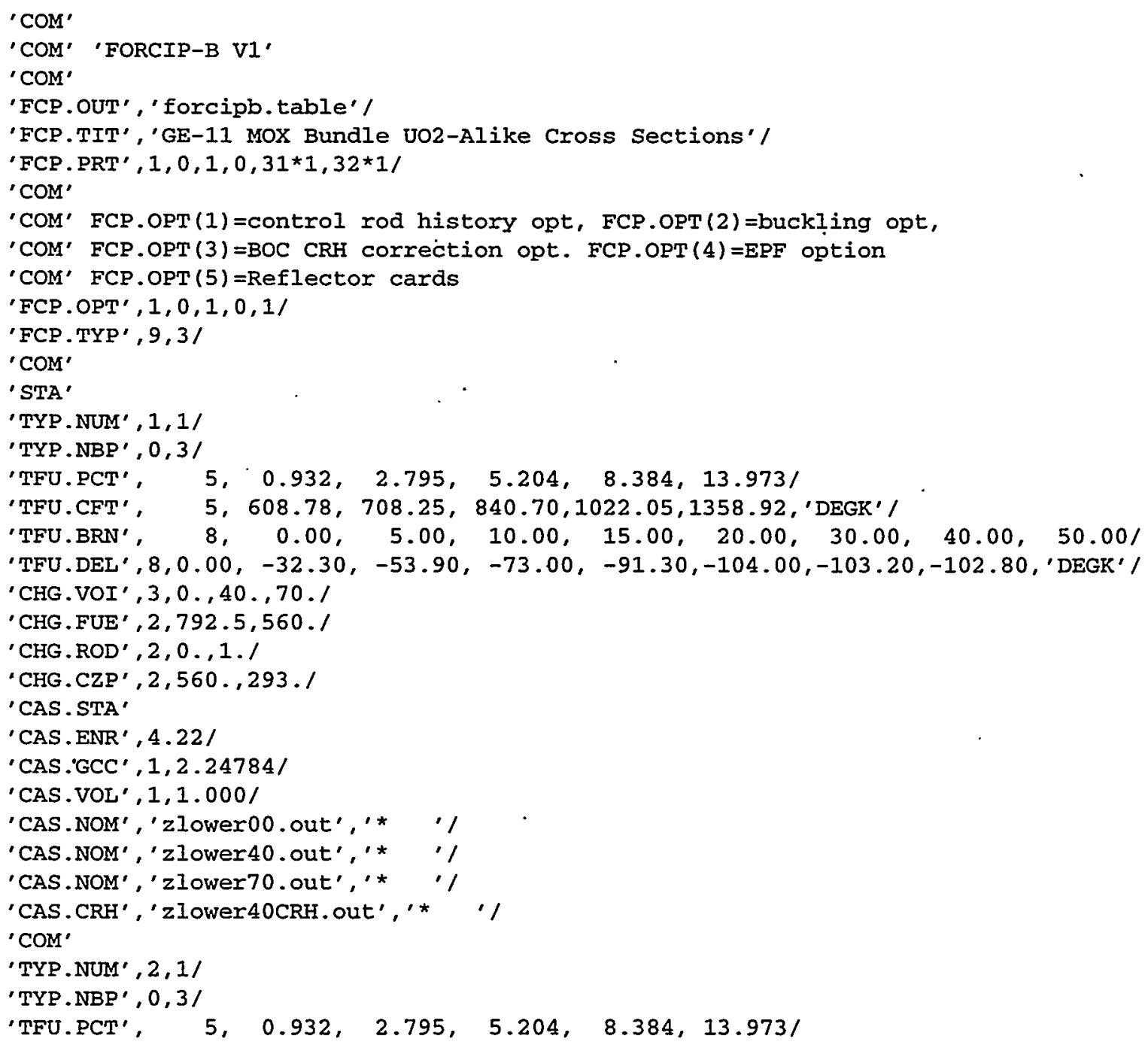




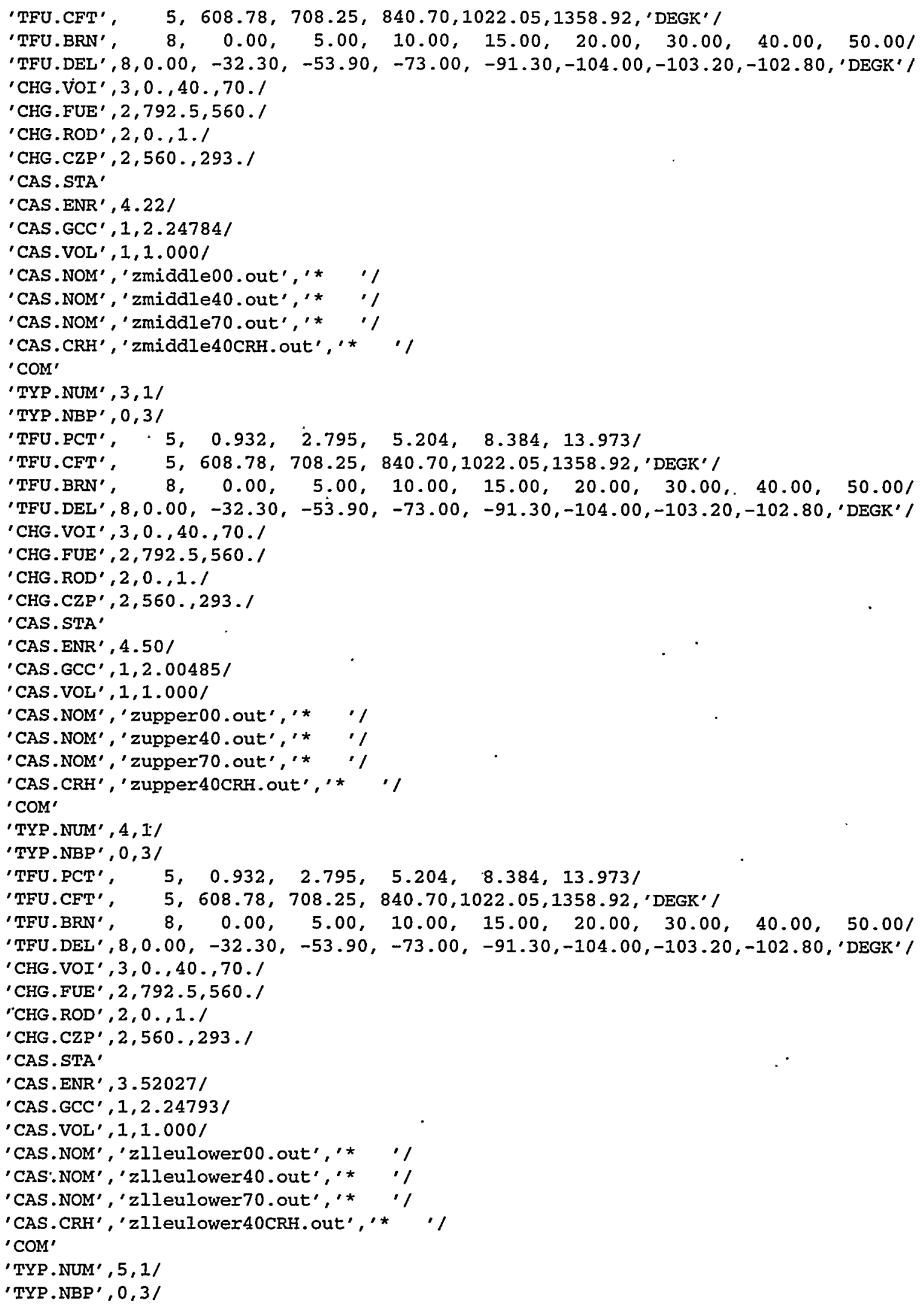




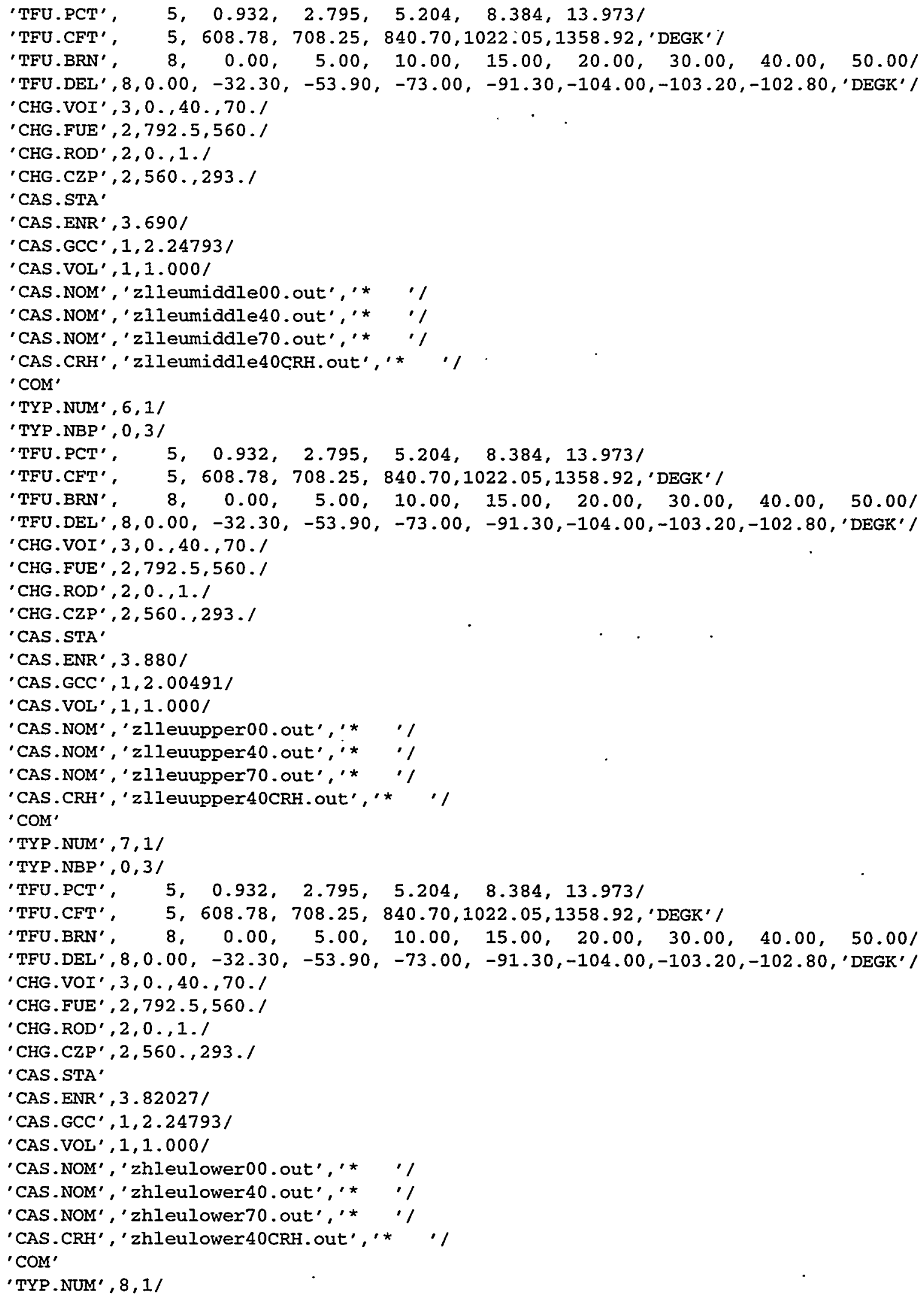




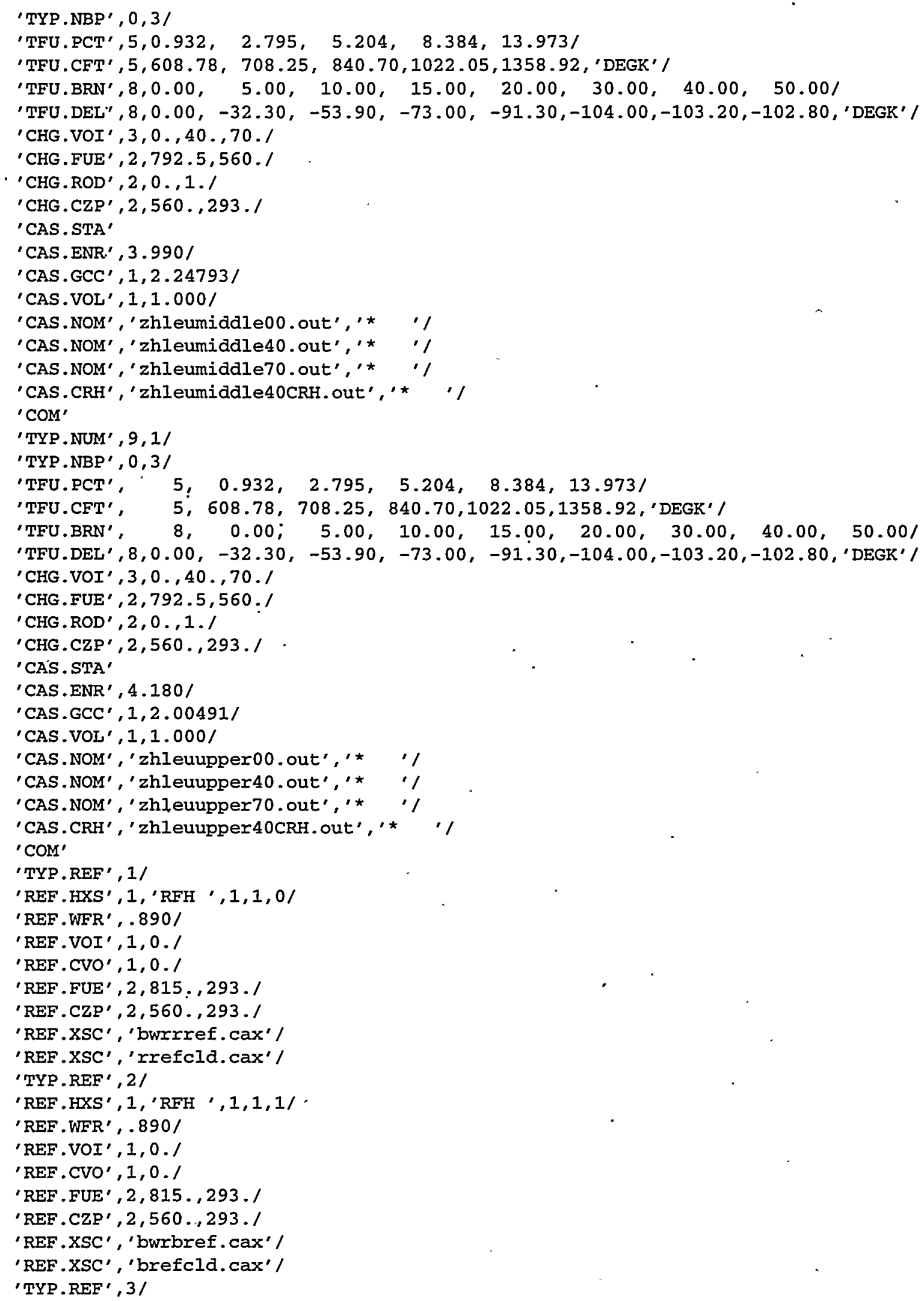


'REF.HXS' , 1, 'RFH ' , 1, 1, 1/

'REF.WFR', . 890/

'REF.VOI', 3,0 .,40.,70.l

'REF.CVO', 1, 0.'

'REF.FUE' , 2, 815 .,293./

'REF.CZP', 2, 560 .,293.l

'REF .XSC', 'bwrtrefo . cax'/

'REF .XSC', 'bwrtref4.cax'/

'REF .XSC', 'bwrtref7.cax'/

'REF.XSC', 'trefcld.cax'/

'END'

/EOF

\section{A4. FORMOSA-B Sample Input File}

The following is a sample of the.FORMOSA-B standard input file for the equilibrium cycle, full MOX core. For complete documentation on the FORMOSA-B standard input file, please refer to [5].

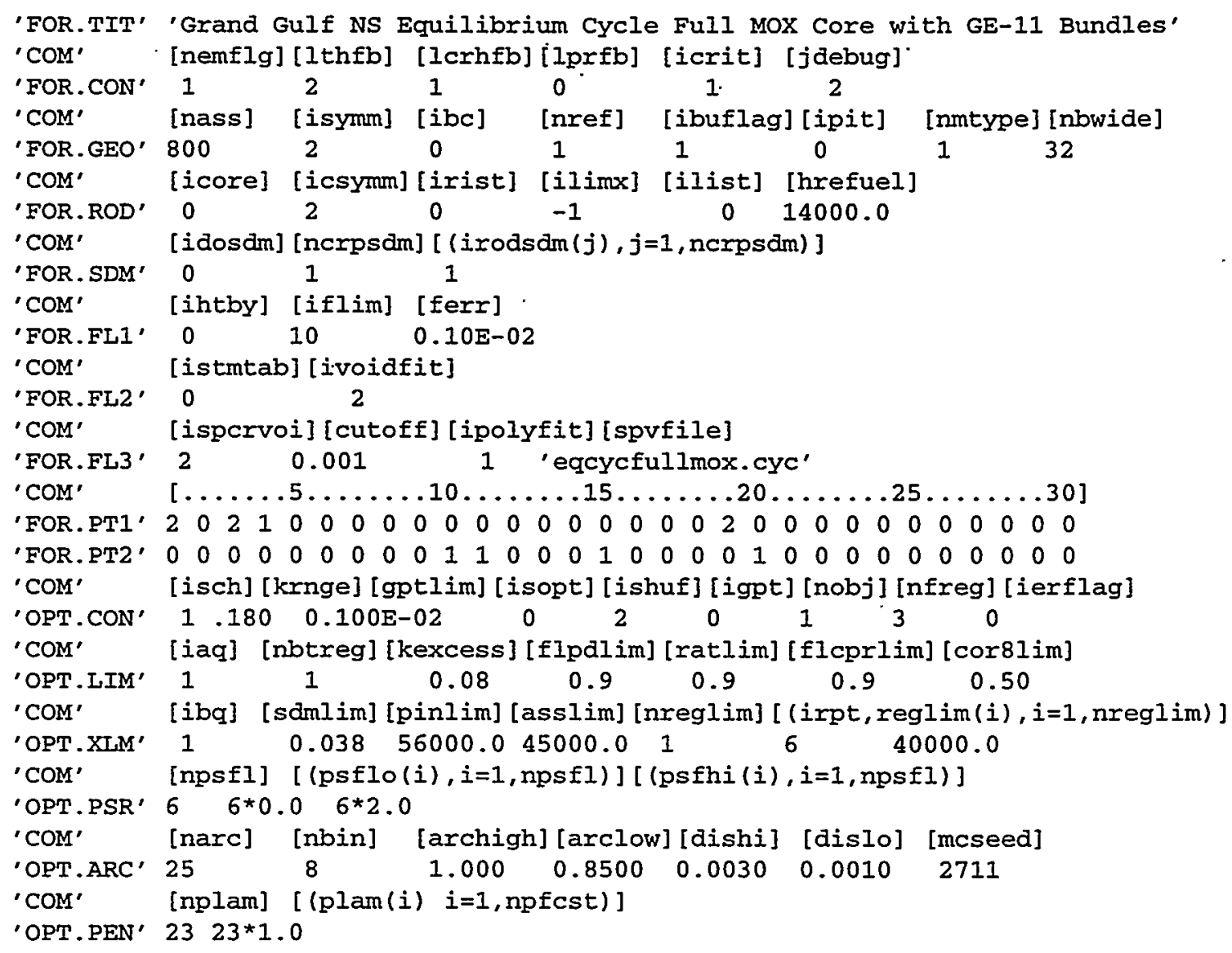




\begin{tabular}{|c|c|c|c|c|c|c|c|c|c|c|c|}
\hline & & & & & & & & & & & \\
\hline 'OPT.DBU' & & & & 1 & 1 & & & & & & \\
\hline 'COM' & & & & & & & & & & & \\
\hline 'FUL . MEC' & & & & mox.cyc' 'IMECH_TYI & & & & & & & \\
\hline ' COM' & & & & reg] [numbat] [fileic & & L)] [file & eidxs (2) & ] [idsu] & ] [ids & & \\
\hline 'FUL. IDS' & 1 & 2 & 1 & 'eqcycfullmox. cyc' & .6 & $53-286$ & $11-286$ & $11-386$ & $53-38^{\prime}$ & 0 & 0 \\
\hline 'FUL. IDS' & 2 & 2 & 1 & 'eqcycfullmox.cyc' & $\cdot 6$ & $51-266$ & $13-266$ & $13-406$ & $51-40^{\prime}$ & & 0 \\
\hline 'FUL.IDS' & 3 & 2 & 1 & 'eqcycfullmox.cyc' & 16 & $35-146$ & $29-146$ & $29-526$ & $35-52^{\prime}$ & 0 & 0 \\
\hline 'FUL. IDS' & 4 & 2 & 1 & 'eqcycfullmox.cyc' & $\cdot 6$ & $37-166$ & $27-166$ & $27-506$ & $37-50^{\prime}$ & 0 & \\
\hline 'FUL.IDS' & 5 & 2 & 1 & 'eqcycfullmox. cyc' & $\cdot 6$ & $51-306$ & $13-306$ & $13-366$ & $51-36^{\prime}$ & & \\
\hline 'FUL. IDS' & 6 & 2 & 1 & 'eqcycfullmox.cyc' & $\cdot 6$ & $51-186$ & $13-186$ & $13-486$ & $51-48^{\prime}$ & & \\
\hline 'FUL.IDS' & 7 & 2 & 1 & 'eqcycfullmox.cyc' & 6 & $49-286$ & $15-286$ & $15-386$ & $49-38^{\prime}$ & & 0 \\
\hline 'FUL.IDS' & 8 & 2 & 1 & 'eqcycfullmox. cyc' & $\cdot 6$ & $49-166$ & $15-166$ & $15-506$ & $49-50^{\prime}$ & 0 & \\
\hline 'FUL. IDS' & 9 & 2 & 1 & 'eqcycfullmox.cyc' & $\cdot 6$ & $53-246$ & $11-246$ & $11-426$ & $53-42^{\prime}$ & & \\
\hline 'FUL.IDS' & 10 & 2 & 1 & 'eqcycfullmox.cyc' & 6 & $47-146$ & $17-146$ & $17-526$ & $47-52$ & & \\
\hline 'FUL.IDS' & 11 & 2 & 1 & 'eqcycfullmox.cyc' & $\cdot 6$ & $39-146$ & $25-146$ & $25-526$ & $39-52$ & & \\
\hline 'EUL. IDS' & 12 & 2 & 1 & 'eqcycfullmox.cyc' & 6 & $43-146$ & $21-146$ & $21-526$ & $43-52$ & & \\
\hline 'EUL.IDS' & 13 & 2 & 1 & 'eqcycfullmox. cyc' & 6 & $37-126$ & $27-126$ & $27-546$ & $37-54^{\prime}$ & & \\
\hline 'EUL. IDS' & $14^{\circ}$ & 1 & 2 & 'eqcycfullmox.cyc' & $\cdot 5$ & $61-325$ & $3-325$ & $3-345$ & $61-34^{\prime}$ & & \\
\hline 'FUL.IDS' & 15 & 1 & 2 & 'eqcycfullmox. cyc' & .5 & $33-045$ & $31-045$ & $31-625$ & $33-62$ ' & & \\
\hline 'FÚL . IDS' & 16 & 2 & 1 & 'eqcycfullmox.cyc' & 16 & $51-226$ & $13-226$ & $13-446$ & $51-44^{\prime}$ & & \\
\hline 'FUL. IDS' & 17 & 2 & 1 & 'eqcycfullmox.cyc' & 16 & $47-266$ & $17-266$ & $17-406$ & $47-40^{\prime}$ & & \\
\hline 'FUL. IDS' & 18 & 2 & 1 & 'eqcycfullmox.cyc' & $\cdot 6$ & $47-226$ & $17-226$ & $17-446$ & $47-44^{\prime}$ & & \\
\hline 'FUL.IDS' & 19 & 2 & 1 & 'eqcycfullmox.cyc' & .6 & $49-246$ & $15-246$ & $15-426$ & $49-42$ & & \\
\hline 'FUL . IDS' & 20 & 2 & 1 & 'eqcycfullmox.cyc' & .6 & $41-126$ & $23-126$ & $23-546$ & $41-54^{\prime}$ & & \\
\hline 'FUL. IDS' & 21 & 2 & 1 & 'eqcycfullmox.cyc' & .6 & $41-166$ & $23-166$ & $23-506$ & $41-50$ & & \\
\hline 'FUL. IDS' & 22 & 2 & 1 & 'eqcycfullmox.cyc' & '6 & $43-186$ & $21-186$ & $21-486$ & $43-48^{\prime}$ & & 0 \\
\hline 'FUL'.IDS' & 23 & 2 & 1 & 'eqcycfullmox.cyc' & .6 & $43-266$ & $21-266$ & $21-406$ & $43-40^{\prime}$ & & \\
\hline 'FUL. IDS' & 24 & 2 & 1 & 'eqcycfullmox. cyc' & 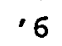 & $39-186$ & $25-186$ & $25-486$ & $39-48^{\prime}$ & & \\
\hline 'FUL. IDS' & 25 & 2 & 1 & 'eqcycfullmox. cyc' & 6 & $53-166$ & $11-166$ & $11-506$ & $53-50^{\prime}$ & & \\
\hline 'FUL. IDS' & 26 & 2 & 1 & 'eqcycfullmox.cyc' & 6 & $53-326$ & $11-326$ & $11-346$ & $53-34$ & & \\
\hline 'FUL. IDS' & 27 & 2 & 1 & 'eqcycfullmox.cyc' & 6 & $53-206$ & $11-206$ & $11-466$ & $53-46^{\prime}$ & & \\
\hline 'FUL. IDS' & 28 & 2 & 1 & 'eqcycfullmox.cyc' & 6 & $41-086$ & $23-086$ & $23-586$ & $41-58^{\prime}$ & & \\
\hline 'FUL . IDS' & 29 & 2 & 1 & 'eqcycfullmox.cyc' & 16 & $55-226$ & $9-226$ & $9-446$ & $55-44^{\prime}$ & $U$ & 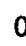 \\
\hline 'FUL . IDS' & 30 & 2 & 1 & 'eqcycfullmox.cyc' & 16 & $33-126$ & $31-126$ & $31-546$ & $33-54^{\prime}$ & & \\
\hline 'FUL. IDS' & 31 & 2 & 1 & 'eqcycfullmox.cyc' & 6 & $43-226$ & $21-226$ & $21-446$ & $43-4$ & & \\
\hline 'FUL. IDS' & 32 & 2 & 1 & 'eqcycfullmox.cyc' & .16 & $49-126$ & $15-126$ & $15-546$ & $49-54^{\prime}$ & 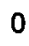 & \\
\hline 'FUL. IDS' & 33 & 2 & 1 & 'eqcycfullmox.cyc' & $\cdot 6$ & $39-226$ & $25-226$ & $25-446$ & $39-44$ & & \\
\hline 'FUL.IDS' & 34 & 2 & 1 & 'eqcycfullmox.cyc' & .6 & $45-126$ & $19-126$ & $19-546$ & $45-54$ & 0 & 0 \\
\hline 'FUL.IDS' & 35 & 2 & 1 & 'eqcycfullmox.cyc' & 6 & $49-326$ & $15-326$ & $15-346$ & $49-34^{\prime}$ & & \\
\hline 'FUL.IDS' & 36 & 2 & 1 & 'eqcycfullmox.cyc' & 6 & $51-146$ & $13-146$ & $13-526$ & $51-52^{\prime}$ & & \\
\hline 'FUL. IDS' & 37 & 2 & 1 & 'eqcycfullmox.cyc' & 6 & $47-306$ & $17-306$ & $17-366$ & $47-36^{\prime}$ & & \\
\hline 'FUL . IDS' & 38 & 2 & 1 & 'eqcycfullmox.cyc' & 6 & $43-106$ & $21-106$ & $21-566$ & $43-56^{\prime}$ & & \\
\hline 'FUL.IDS'. & 39 & 2 & 1 & 'eqcycfullmox.cyc' & 6 & $57-246$ & $7-246$ & $7-426$ & $57-42^{\prime}$ & 0 & 0 \\
\hline 'FUL. IDS' & 40 & 2 & 1 & 'eqcycfullmox.cyc' & 6 & $35-306$ & $29-306$ & $29-366$ & $35-36^{\prime}$ & & \\
\hline 'FUL . IDS' & 41 & 2 & 1 & 'eqcycfullmox.cyc' & 16 & $35-186$ & $29-186$ & $29-486$ & $35-48^{\prime}$ & & \\
\hline 'FUL.IDS' & 42 & 2 & 1 & 'eqcycfullmox.cyc' & 6 & $57-286$ & $7-286$ & $7-386$ & $57-38^{\prime}$ & & \\
\hline 'FUL. IDS' & 43 & 2 & 1 & 'eqcycfullmox.cyc' & 6 & $33-086$ & $31-086$ & $31-586$ & $33-58^{\prime}$ & & \\
\hline 'FUL. IDS' & 44 & 2 & 1 & 'eqcycfullmox.cyc' & 16 & $33-166$ & $31-166$ & $31-506$ & $33-50^{\prime}$ & & \\
\hline 'FUL. IDS' & 45 & 2 & 1 & 'eqcycfullmox.cyc' & 16 & $39-266$ & $25-266$ & $25-406$ & $39-40^{\prime}$ & & \\
\hline 'FUL . IDS' & 46 & 2 & 1 & 'eqcycfullmox. cyc' & 6 & $57-326$ & $7-326$ & $7-346$ & $57-34^{\prime}$ & & \\
\hline 'FUL.IDS' & 47 & 2 & 1 & 'eqcycfullmox.cyc' & 6 & $37-086$ & $27-086$ & $27-586$ & $37-58^{\prime}$ & & \\
\hline 'FUL.IDS' & 48 & 2 & 1 & 'eqcycfullmox.cyc' & 16 & $45-166$ & $19-166$ & $19-506$ & $45-50^{\prime}$ & & \\
\hline 'FUL. IDS' & 49 & 2 & 1 & 'eqcycfullmox.cyc' & $\cdot 6$ & $49-206$ & $15-206$ & $15-466$ & $49-46^{\prime}$ & & \\
\hline 'FUL . IDS' & 50 & 2 & 1 & 'eqcycfullmox.cyc' & 16 & $35-226$ & $29-226$ & $29-446$ & $35-44^{\prime}$ & & \\
\hline 'FUL . IDS' & 51 & 2 & 1 & 'eqcycfullmox.cyc' & $\cdot 6$ & $43-306$ & $21-306$ & $21-366$ & $43-36^{\prime}$ & 0 & c \\
\hline
\end{tabular}




\begin{tabular}{|c|c|c|c|c|c|c|c|c|c|c|}
\hline 'FUL. IDS' & 52 & 2 & 1 & 'eqcycfullmox.cyc' & 6 & $53-126$ & $11-126$ & $11-546$ & $53-54$ & \\
\hline 'FUL. IDS' & 53 & 2 & 1 & 'eqcycfullmox. cyc' & $\cdot 6$ & $47-186$ & $17-186$ & $17-486$ & $47-48^{\prime}$ & \\
\hline 'FUL. IDS' & 54 & 2 & 1 & ' eqcycfullmox.cyc' & 6 & $55-266$ & $9-266$ & $9-406$ & $55-40^{\prime}$ & \\
\hline 'FUL . IDS' & 55 & 2 & 1 & 'eqcycfullmox.cyc' & 16 & $45-206$ & $19-206$ & $19-466$ & $45-46^{\prime}$ & \\
\hline 'FU். IDS' & 56 & 2 & 1 & eqcycfullmox.cyc' & 16 & $39-106$ & $25-106$ & $25-566$ & $39-56^{\prime}$ & 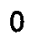 \\
\hline 'FUL . IDS' & 57 & 2 & 1 & eqcycfullmox. cyc' & 6 & $35-266$ & $29-266$ & $29-406$ & $5-40^{\prime}$ & 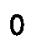 \\
\hline 'FUL . IDS' & 58 & 2. & 1 & eqcycfullmox.cyc' & .6 & $55-306$ & $9-306$ & $9-366$ & $55-36$ & 0 \\
\hline 'FUL. IDS' & 59 & 2 & 1 & eqcycfullmox.cyc' & 16 & $35-106$ & $29-106$ & $29-566$ & $35-56^{\prime}$ & 0 \\
\hline 'FUL. IDS' & 60 & 2 & 1 & eqcycfullmox. cyc: & 16 & $39-306$ & $25-306$ & $25-366$ & $39-36^{\prime}$ & 0 \\
\hline 'FUL. IDS' & 61 & 2 & 1 & eqcycfullmox.cyc' & $\cdot 6$ & $47-106$ & $17-106$ & $17-566$ & $47-56^{\prime}$ & 0 \\
\hline 'FUL. IDS'. & 62 & 2 & 1 & eqcycfullmox.cyc' & $\cdot 6$ & $55-186$ & $9-186$ & $9-486$ & $55-48^{\prime}$ & 0 \\
\hline 'FUL. IDS' & 63 & 3 & 1 & eqcycfullmox.cyc' & 7 & $43-227$ & $21-227$ & $21-447$ & $43-44^{\prime}$ & \\
\hline 'FUL. IDS' & 64 & 3 & 1 & eqcycfullmox.cyc' & 7 & $45-207$ & $19-207$ & $19-467$ & $45-46^{\prime}$ & \\
\hline 'FUL . IDS' & 65 & 2 & 1 & eqcycfullmox.cyc' & .6 & $57-206$ & $7-206$ & $7-466$ & $57-46^{\prime}$ & \\
\hline 'FUL . IDS' & 66 & 2 & 1 & eqcycfullmox.cyc' & .6 & $59-306$ & $5-306$ & $5-366$ & $59-36^{\prime}$ & 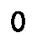 \\
\hline 'FUL. IDS' & 67 & 2 & 1 & eqcycfullmox.cyc' & $\cdot 6$ & $45-086$ & $19-086$ & $19-586$ & $45-58^{\prime}$ & 0 \\
\hline 'FUL. IDS' & 68 & 2 & 1 & eqcycfullmox.cyc' & $\cdot 6$ & $35-c$ & $29-066$ & $29-606$ & $35-60^{\circ}$ & \\
\hline 'FUL . IDS' & 69 & 3 & 1 & eqcycfullmox.cyc' & 7 & $39-227$ & $25-227$ & $25-447$ & $39-44^{\prime}$ & 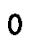 \\
\hline 'FUL. IDS' & 70 & 3 & 1 & eqcycfullmox.cyc' & $\cdot 7$ & $49-207$ & $15-207$ & $15-467$ & $49-46^{\prime}$ & \\
\hline 'FUL. IDS' & 71 & 3 & 1 & eqcycfullmox.cyc' & 7 & $39-147$ & $25-147$ & $25-527$ & $39-52^{\prime}$ & 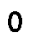 \\
\hline 'FUL . IDS' & 72 & 3 & 1 & eqcycfullmox.cyc' & 17 & $41-127$ & $23-127$ & $23-547$ & $41-54^{\prime}$ & 0 \\
\hline 'FUL.IDS' & 73 & 3 & 1 & eqcycfulimox.cyc' & 7 & 227 & $17-227$ & $17-447$ & $47-44^{\prime}$ & 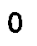 \\
\hline 'FUL. IDS' & 74 & 3 & 1 & eqcycfullmox.cyc' & 7 & $39-267$ & $25-267$ & $25-407$ & $-40^{\prime}$ & \\
\hline 'FUL. IDS' & 75 & 3 & 1 & eqcycfullmox.cyc' & 7 & $33-047$ & $31-047$ & $31-627$ & $33-62$ & 0 \\
\hline 'FUL.IDS' & 76 & 3 & 1 & eqcycfullmox.cyc' & 7 & $61-327$ & $3-327$ & $3-347$ & & 0 \\
\hline 'FUL. IDS' & 77 & 2 & 2 & 'eqcycfullmox.cyc' & $\cdot 6$ & $61-326$ & $3-326$ & $3-346$ & $61-34^{\prime}$ & 0 \\
\hline 'FUL. IDS' & 78 & 3 & 1 & 'eqcycfullmox.cyc' & 17 & $43-267$ & $21-267$ & $21-407$ & $43-40^{\prime}$ & \\
\hline 'FUL . IDS' & 79 & 2 & 2 & eqcycfullmox.cyc' & 16 & $33-046$ & $31-046^{\circ}$ & $31-626$ & $33-62^{\prime}$ & U \\
\hline 'FUL . IDS' & 80 & 3 & 1 & 'eqcycfulimox.cyc' & $\cdot 7$ & $49-167$ & $15-167$ & $15-507$ & $49-50^{\prime}$ & \\
\hline 'FUI . IDS' & 81 & 3 & 1 & 'eqcycfulimox.cyc' & $\cdot 7$ & $51-267$ & $13-267$ & $13-407$ & $51-40^{\prime}$ & \\
\hline 'FUI. IDS' & 82 & 3 & 1 & 'eqcycfulimox.cyc' & 17 & $45-167$ & $19-167$ & $19-507$ & $45-50^{\prime}$ & \\
\hline 'FUL. IDS' & 83 & 3 & 1 & 'eqcycfulimox.cyc' & $\cdot 7$ & $47-187$ & $17-187$ & $17-487$ & $47-48^{\prime}$ & \\
\hline 'FUL. IDS' & 84 & 3 & 1 & 'eqcycfullmox.cyc' & $\cdot 7$ & $35-267$ & $29-267$ & $29-407$ & $35-40^{\prime}$ & \\
\hline 'FUL . IDS' & 85 & 3 & 1 & 'eqcycfullmox.cyc' & 7 & $35-307$ & $29-307$ & $29-367$ & $35-36^{\prime}$ & c \\
\hline 'FUL. IDS' & 86 & 3 & 1 & 'eqcycfullmox.cyc' & $\cdot 7$ & & $17-147$ & $17-527$ & $52^{\prime}$ & \\
\hline 'FUL.IDS' & 87 & 3 & 1 & 'eqcycfullmox.cyc' & 7 & $51-227$ & $13-227$ & $13-447$ & $51-44^{\prime}$ & \\
\hline 'FUL. IDS' & 88 & 3 & 1 & 'eqcycfullmox.cyc' & 7 & $53-247$ & $11-247$ & $11-427$ & $53-42^{\prime}$ & \\
\hline 'FUL. IDS' & 89 & 3 & 1 & 'eqcycfullmox.cyc' & $\cdot 7$ & $49-247$ & $15-247$ & $15-427$ & $49-42^{\prime}$ & \\
\hline 'FUL. IDS' & 90 & 3 & 1 & 'eqcycfullmox.cyc' & 17 & $39-307$ & $25-307$ & $25-367$ & $39-36^{\prime}$ & \\
\hline 'FUL. IDS' & 91 & 3 & 1 & 'eqcycfullmox.cyc' & $\cdot 7$ & $49-$ & $15-287$ & $15-387$ & $49-38^{\prime}$ & \\
\hline 'FUL. IDS' & 92 & 3 & 1 & 'eqcycfullmox.cyc' & $\cdot 7$ & $51-187$ & $13-187$ & $13-487$ & $51-48^{\prime}$ & \\
\hline 'FUL. IDS' & 93 & 3 & 1 & 'eqcycfullmox.cyc' & 7 & $53-287$ & $11-287$ & $11-387$ & $53-38^{\prime}$ & \\
\hline 'FUL. IDS' & 94 & 3. & 1 & 'eqcycfullmox. cyc' & $\cdot 7$ & $47-267$ & $17-267$ & $17-407$ & $47-40^{\prime}$ & \\
\hline 'FUL. IDS' & 95 & 3 & 1 & 'eqcycfullmox.cyc' & $\cdot 7$ & $43-147$ & $21-147$ & $21-527$ & $43-52^{\prime}$ & \\
\hline 'FUL. IDS' & 96 & 3 & 1 & 'eqcycfullmox.cyc' & 7 & $41-167$ & $23-167$ & $23-507$ & $41-50^{\prime}$ & \\
\hline 'FUL . IDS' & 97 & 3 & 1 & ' eqcycfullmox.cyc' & 7 & $51-307$ & $13-307$ & $13-367$ & $51-36^{\prime}$ & \\
\hline 'FUL . IDS' & 98 & 3 & 1 & 'eqcycfullmox.cyc' & 7 & $37-167$ & $27-167$ & $27-507$ & $37-50^{\prime}$ & \\
\hline 'FUL. IDS' & 99 & 3 & 1 & 'eqcycfullmox.cyc' & 17 & $49-327$ & $15-327$ & $15-347$ & $49-34^{\prime}$ & \\
\hline 'FUL. IDS' & 100 & 3 & 1 & 'eqcycfullmox.cyc' & $\cdot 7$ & $53-207$ & $11-207$ & $11-467$ & $53-46^{\prime}$ & \\
\hline 'FUL . IDS' & 101 & 3 & 1 & 'eqcycfullmox.cyc' & 7 & $45-127$ & $19-127$ & $19-547$ & $45-54^{\prime}$ & \\
\hline 'FUL. IDS' & 102 & 3 & 1 & 'eqcycfullmox.cyc' & 7 & $39-187$ & $25-187$ & $25-487$ & $39-48^{\prime}$ & \\
\hline 'FUL. IDS' & 103 & 3 & 1 & 'eqcycfullmox. cyc' & 7 & $35-147$ & $29-147$ & $29-527$ & $35-52^{\prime}$ & \\
\hline 'FUL. IDS' & 104 & 3 & 1 & 'eqcycfullmox. cyc' & $\cdot 7$ & $37-127$ & $27-127$ & $27-547$ & $37-54^{\prime}$ & 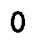 \\
\hline 'FUI. IDS' & 105 & 3 & 1 & 'eqcycfullmox.cyc' & 7 & $43-187$ & $21-187$ & $21-487$ & $43-48^{\prime}$ & \\
\hline 'FUL. IDS' & 106 & 3 & 1 & 'eqcycfullmox.cyc' & 7 & $55-267$ & $9-267$ & $9-407$ & $55-40^{\prime}$ & 0 \\
\hline 'FUL. IDS' & 107 & 3 & 1 & 'eqcycfullmox.cyc' & 7 & $47-307$ & $17-307$ & $17-367$ & $47-36^{\prime}$ & 0 \\
\hline
\end{tabular}




\begin{tabular}{|c|c|c|c|c|c|c|c|c|c|}
\hline 'FUL. IDS' 108 & 3 & 1 & 'eqcycfullmox.cyc' & 7 & $33-167$ & $31-167$ & $31-507$ & $33-50^{\prime}$ & $n$ \\
\hline 'FUL. IDS' 109 & 3 & 1 & 'eqcycfullmox.cyc' & 7 & $53-327$ & $11-327$ & $11-347$ & $53-34^{\prime}$ & \\
\hline 'FUL. IDS' 110 & & & 'eqcycfullmox.cyc' & 77 & $35-187$ & $29-187$ & $29-487$ & $35-48^{\prime}$ & \\
\hline 'FUL. IDS' 111 & 3 & $\perp$ & 'eqcycfull mox.cyc' & 17 & $55-307$ & $9-307$ & $9-367$ & $55-36^{\prime}$ & \\
\hline 'FUL. IDS' 112 & 3 & 1 & 'eqcycfulimox.cyc' & 17 & $51-147$ & $13-147$ & $13-527$ & $51-52^{\prime}$ & \\
\hline 'FUL. IDS' 113 & 3 & $\perp$ & 'eqcycfullmox. cyc' & 7 & $5-227$ & $9-227$ & $9-447$ & $55-44^{\prime}$ & \\
\hline 'FUL.IDS' 114 & 3 & & 'eqcycfullmox. cyc' & 17 & $33-127$ & $31-127$ & $31-547$ & $33-54$ & \\
\hline 'FUL.IDS' 115 & 3 & & 'eqcycfullmox.cyc' & 17 & $39-107$ & $25-107$ & $25-567$ & $39-56^{\prime}$ & \\
\hline 'FUL. IDS'116 & 3 & & 'eqcycfullmox.cyc' & 7 & $35-107$ & $29-107$ & $29-567$ & $35-56^{\prime}$ & \\
\hline 'FUL. IDS' 117 & 3 & & 'eqcycfullmox.cyc' & .7 & $35-227$ & $29-227$ & $29-447$ & $35-44^{\prime}$ & \\
\hline 'FUL . IDS' 118 & 3 & 1 & 'eqcycfullmox. cyc' & 7 & $43-1.07$ & $21-107$ & $21-567$ & $43-56^{\prime}$ & \\
\hline 'FUL. IDS' 119 & 3 & 1 & 'eqcycfullmox.cyc' & 7 & $49-127$ & $15-127$ & $15-547$ & $49-54^{\prime}$ & \\
\hline 'FUL. IDS' 120 & 3 & 1 & 'eqcycfullmox.cyc' & 7 & $53-167$ & $11-167$ & $11-507$ & $53-50^{\prime}$ & \\
\hline 'FUL. IDS' 121 & 3 & 1 & 'eqcycfullmox.cyc' & 7 & $53-127$ & $11-127$ & $11-547$ & $-54^{\prime}$ & \\
\hline 'FUI. IDS' 122 & 3 & 1 & 'eqcycfullmox.cyc' & 17 & $43-307$ & $21-307$ & $21-367$ & $43-36^{\prime}$ & \\
\hline 'FUL. IDS' 123 & 3 & 1 & 'eqcycfullmox.cyc' & 7 & $57-327$ & $7-327$ & $7-347$ & $57-34^{\prime}$ & \\
\hline 'FUL. IDS' 124 & 3 & 1 & 'eqcycfullmox. cyc' & 7 & $35-067$ & $29-067$ & $29-607$ & $35-60^{\prime}$ & \\
\hline 'EUL. IDS' 125 & 3 & & 'eqcycfullmox.cyc' & 7 & $33-087$ & $31-087$ & $31-587$ & $33-58^{\prime}$ & \\
\hline 'FUL.IDS' 126 & 3 & 1 & 'eqcycfullmox.cyc' & 7 & $57-287$ & $7-287$ & $7-387$ & $57-38^{\prime}$ & \\
\hline 'FUL. IDS' 127 & 3 & & 'eqcycfullmox.cyc' & .7 & $59-307$ & $5-307$ & $5-367$ & $59-36^{\prime}$ & \\
\hline 'FUL. IDS' 128 & 3 & 1 & 'eqcycfullmox.cyc' & 7 & $47-107$ & $17-107$ & $17-567$ & $47-56^{\prime}$ & 0 \\
\hline 'FUL . IDS' 129 & 3 & 1 & 'eqcycfullmox.cyc' & 7 & $37-087$ & $27-087$ & $27-587$ & $37-58^{\prime}$ & \\
\hline 'FUL. IDS' 130 & 3 & 1 & 'eqcycfullmox.cyc' & 7 & $55-187$ & $9-187$ & $9-487$ & $55-48^{\prime}$ & 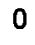 \\
\hline 'FUL.IDS' 131 & 3 & 1 & 'eqcycfullmox.cyc' & 17 & $45-087$ & $19-087$ & $19-587$ & $45-58^{\prime}$ & 0 \\
\hline 'FUL. IDS' 132 & 3 & 1 & 'eqcycfullmox.cyc' & 17 & $57-207$ & $7-207$ & $7-467$ & $57-46^{\prime}$ & \\
\hline 'FUL. IDS' 133 & 3 & 1 & 'eqcycfull Imox.cyc' & 7 & $57-247$ & $7-247$ & $7-427$ & $57-42^{\prime}$ & 0 \\
\hline 'FUL . IDS' 134 & 3 & 1 & 'eqcycfullmox.cyc' & 17 & $41-087$ & $23-087$ & $23-587$ & $41-58^{\prime}$ & 0 \\
\hline 'FUL. IDS' 135 & 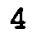 & 1 & 'eqcycfullmox.cyc' & & & $572 \pi$ & 70 LYV57 & $1-M O X$ & 0 \\
\hline
\end{tabular}

\section{'MAP.FLP'}

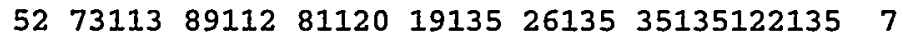

$\begin{array}{llllllll}105135 & 27135 & 18135 & 46135 & 36135 & 49135 & 60135127 & 14\end{array}$

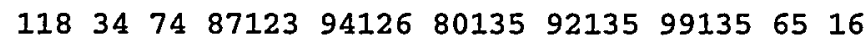

. $96135951353713542135291355113562106 \quad 6$

$\begin{array}{llllllll}85 & 22125 & 41121 & 91133 & 88135 & 97135100135 & 64 & 8\end{array}$

$\begin{array}{lllllll}71135102135 & 98135 & 39135 & 25135 & 54135 & 66111 & 23\end{array}$

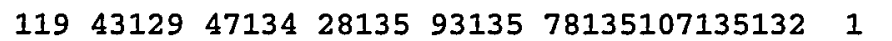

$21135 \quad 631357213510413555135 \quad 58135130 \quad 5$

$\begin{array}{llllll}135 & 40135 \quad 38135 \quad 32135 \cdot 53135109135 & 70 & 2\end{array}$

$\begin{array}{llllll}30135 & 86135103135 & 69135114135 & 77 & 90 & 17\end{array}$

$\begin{array}{llllllll}135 & 48135 & 50135 & 56135 & 59135 & 79135 & 76 & 9\end{array}$

$\begin{array}{llllll}44135108135101135110135 & 82 & 84 & 75 & 45\end{array}$

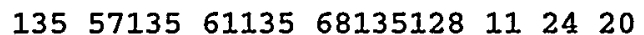

$\begin{array}{llll}117135 & 67115 & 83116131 & 3\end{array}$

$\begin{array}{lllllll}135124 & 12 & 10 & 31 & 33 & 13^{\circ}\end{array}$.

415

'MAP.SLP', Initial Fuel Subshade Pattern (fixed-format)

$\begin{array}{llllllllllllllll}0 & 0 & 0 & 0 & 0 & 0 & 0 & 0 & 0 & 0 & 0 & 0 & 0 & 0 & 0 & 0 \\ 0 & 0 & 0 & 0 & 0 & 0 & 0 & 0 & 0 & 0 & 0 & 0 & 0 & 0 & 0 & 0 \\ 0 & 0 & 0 & 0 & 0 & 0 & 0 & 0 & 0 & 0 & 0 & 0 & 0 & 0 & 0 & \\ 0 & 0 & 0 & 0 & 0 & 0 & 0 & 0 & 0 & 0 & 0 & 0 & 0 & 0 & 0 & \\ 0 & 0 & 0 & 0 & 0 & 0 & 0 & 0 & 0 & 0 & 0 & 0 & 0 & 0 & 0 & \\ 0 & 0 & 0 & 0 & 0 & 0 & 0 & 0 & 0 & 0 & 0 & 0 & 0 & 0 & 0 & \\ 0 & 0 & 0 & 0 & 0 & 0 & 0 & 0 & 0 & 0 & 0 & 0 & 0 & 0 & 0 & \\ 0 & 0 & 0 & 0 & 0 & 0 & 0 & 0 & 0 & 0 & 0 & 0 & 0 & 0 & & \\ 0 & 0 & 0 & 0 & 0 & 0 & 0 & 0 & 0 & 0 & 0 & 0 & 0 & & & \\ 0 & 0 & 0 & 0 & 0 & 0 & 0 & 0 & 0 & 0 & 0 & 0 & 0 & & & \end{array}$




$$
\begin{array}{lllllllllllll}
0 & 0 & 0 & 0 & 0 & 0 & 0 & 0 & 0 & 0 & 0 & 0 & 0 \\
0 & 0 & 0 & 0 & 0 & 0 & 0 & 0 & 0 & 0 & 0 & 0 & \\
0 & 0 & 0 & 0 & 0 & 0 & 0 & 0 & 0 & 0 & 0 & & \\
0 & 0 & 0 & 0 & 0 & 0 & 0 & 0 & & & & & \\
0 & 0 & 0 & 0 & 0 & 0 & 0 & & & & & & \\
0 & 0 & & & & & & & & & & &
\end{array}
$$

'MAP.ROT', Initial Orientation Pattern (fixed-format)

$$
\begin{array}{llllllllllllllll}
0 & 1 & 0 & 1 & 0 & 1 & 0 & 1 & 0 & 1 & 0 & 1 & 0 & 1 & 0 & 1
\end{array}
$$

$\begin{array}{llllllllllllllll}3 & 2 & 3 & 2 & 3 & 2 & 3 & 2 & 3 & 2 & 3 & 2 & 3 & 2 & 3 & 2\end{array}$

$\begin{array}{lllllllllllllll}0 & 1 & 0 & 1 & 0 & 1 & 0 & 1 & 0 & 1 & 0 & 1 & 0 & 1 & 0\end{array}$

$\begin{array}{lllllllllllllll}3 & 2 & 3 & 2 & 3 & 2 & 3 & 2 & 3 & 2 & 3 & 2 & 3 & 2 & 3\end{array}$

$\begin{array}{lllllllllllllll}0 & 1 & 0 & 1 & 0 & 1 & 0 & 1 & 0 & 1 & 0 & 1 & 0 & 1 & 0\end{array}$

$\begin{array}{lllllllllllllll}3 & 2 & 3 & 2 & 3 & 2 & 3 & 2 & 3 & 2 & 3 & 2 & 3 & 2 & 3\end{array}$

$\begin{array}{lllllllllllllll}0 & 1 & 0 & 1 & 0 & 1 & 0 & 1 & 0 & 1 & 0 & 1 & 0 & 1 & 0\end{array}$

$\begin{array}{llllllllllllll}3 & 2 & 3 & 2 & 3 & 2 & 3 & 2 & 3 & 2 & 3 & 2 & 3 & 2\end{array}$

$\begin{array}{lllllllllllll}0 & 1 & 0 & 1 & 0 & 1 & 0 & 1 & 0 & 1 & 0 & 1 & 0\end{array}$

$\begin{array}{lllllllllllll}3 & 2 & 3 & 2 & 3 & 2 & 3 & 2 & 3 & 2 & 3 & 2 & 3\end{array}$

$\begin{array}{lllllllllllll}0 & 1 & 0 & 1 & 0 & 1 & 0 & 1 & 0 & 1 & 0 & 1 & 0\end{array}$

$\begin{array}{llllllllllll}3 & 2 & 3 & 2 & 3 & 2 & 3 & 2 & 3 & 2 & 3 & 2\end{array}$

$\begin{array}{lllllllllll}0 & 1 & 0 & 1 & 0 & 1 & 0 & 1 & 0 & 1 & 0\end{array}$

$\begin{array}{llllllll}3 & 2 & 3 & 2 & 3 & 2 & 3 & 2\end{array}$

$\begin{array}{lllllll}0 & 1 & 0 & 1 & 0 & 1 & 0\end{array}$

$\begin{array}{ll}3 & 2\end{array}$

'MAP.ROD', Control Rod Identification Array (fixed-format)

$$
\begin{array}{rrrrrrrrrrrrrrrr}
1 & 2 & 2 & 3 & 3 & 4 & 4 & 5 & 5 & 6 & 6 & 7 & 7 & 8 & 8 & 0 \\
9 & 10 & 10 & 11 & 11 & 12 & 12 & 13 & 13 & 14 & 14 & 15 & 15 & 16 & 16 & 0
\end{array}
$$

$\begin{array}{lllllllllllllll}9 & 10 & 10 & 11 & 11 & 12 & 12 & 13 & 13 & 14 & 14 & 15 & 15 & 16 & 16\end{array}$

$\begin{array}{lllllllllllllll}17 & 18 & 18 & 19 & 19 & 20 & 20 & 21 & 21 & 22 & 22 & 23 & 23.24 & 24\end{array}$

$\begin{array}{lllllllllllllll}17 & 18 & 18 & 19 & 19 & 20 & 20 & 21 & 21 & 22 & 22 & 23 & 23 & 24 & 24\end{array}$

$\begin{array}{lllllllllllllll}25 & 26 & 26 & 27 & 27 & 28 & 28 & 29 & 29 & 30 & 30 & 31 & 31 & 32 & 32\end{array}$

$\begin{array}{lllllllllllllll}25 & 26 & 26 & 27 & 27 & 28 & 28 & 29 & 29 & 30 & 30 & 31 & 31 & 32 & 32\end{array}$

$\begin{array}{llllllllllllll}33 & 34 & 34 & 35 & 35 & 36 & 36 & 37 & 37 & 38 & 38 & 39 & 39 & 0\end{array}$

$\begin{array}{lllllllllllll}33 & 34 & 34 & 35 & 35 & 36 & 36 & 37 & 37 & 38 & 38 & 39 & 39\end{array}$

$\begin{array}{lllllllllllll}40 & 41 & 41 & 42 & 42 & 43 & 43 & 44 & 44 & 45 & 45 & 46 & 46\end{array}$

$\begin{array}{lllllllllllll}40 & 41 & 41 & 42 & 42 & 43 & 43 & 44 & 44 & 45 & 45 & 46 & 46\end{array}$

$\begin{array}{llllllllllll}47 & 48 & 48 & 49 & 49 & 50 & 50 & 51 & 51 & 52 & 52 & 0\end{array}$

$\begin{array}{lllllllllll}47 & 48 & 48 & 49 & 49 & 50 & 50 & 51 & 51 & 52 & 52\end{array}$

$\begin{array}{llllllll}53 & 54 & 54 & 55 & 55 & 56 & 56 & 0\end{array}$

$\begin{array}{lllllll}53 & 54 & 54 & 55 & 55 & 56 & 56\end{array}$

00

'MAP.ORF', Hydraulic Throttling Map (fixed-format)

$\begin{array}{llllllllllllllll}1 & 1 & 1 & 1 & 1 & 1 & 1 & 1 & 1 & 1 & 1 & 1 & 1 & 1 & 1 & 2\end{array}$

$\begin{array}{llllllllllllllll}1 & 1 & 1 & 1 & 1 & 1 & 1 & 1 & 1 & 1 & 1 & 1 & 1 & 1 & 1 & 2\end{array}$

$\begin{array}{lllllllllllllll}1 & 1 & 1 & 1 & 1 & 1 & 1 & 1 & 1 & 1 & 1 & 1 & 1 & 1 & 2\end{array}$

$\begin{array}{lllllllllllllll}1 & 1 & 1 & 1 & 1 & 1 & 1 & 1 & 1 & 1 & 1 & 1 & 1 & 1 & 2\end{array}$

$\begin{array}{lllllllllllllll}1 & 1 & 1 & 1 & 1 & 1 & 1 & 1 & 1 & 1 & 1 & 1 & 1 & 1 & 2\end{array}$

$\begin{array}{lllllllllllllll}1 & 1 & 1 & 1 & 1 & 1 & 1 & 1 & 1 & 1 & 1 & 1 & 1 & 1 & 2\end{array}$

$\begin{array}{lllllllllllllll}1 & 1 & 1 & 1 & 1 & 1 & 1 & 1 & 1 & 1 & 1 & 1 & 1 & 1 & 2\end{array}$

$\begin{array}{llllllllllllll}1 & 1 & 1 & 1 & 1 & 1 & 1 & 1 & 1 & 1 & 1 & 1 & 1 & 2\end{array}$

$\begin{array}{lllllllllllll}1 & 1 & 1 & 1 & 1 & 1 & 1 & 1 & 1 & 1 & 1 & 1 & 2\end{array}$

$\begin{array}{lllllllllllll}1 & 1 & 1 & 1 & 1 & 1 & 1 & 1 & 1 & 1 & 1 & 1 & 2\end{array}$

$\begin{array}{lllllllllllll}1 & 1 & 1 & 1 & 1 & 1 & 1 & 1 & 1 & 1 & 1 & 1 & 2\end{array}$

$\begin{array}{llllllllllll}1 & 1 & 1 & 1 & 1 & 1 & 1 & 1 & 1 & 1 & 1 & 2\end{array}$

$\begin{array}{lllllllllll}1 & 1 & 1 & 1 & 1 & 1 & 1 & 1 & 2 & 2 & 2\end{array}$

$\begin{array}{llllllll}1 & 1 & 1 & 1 & 1 & 1 & 1 & 2\end{array}$

$\begin{array}{lllllll}1 & 1 & 2 & 2 & 2 & 2 & 2\end{array}$ 


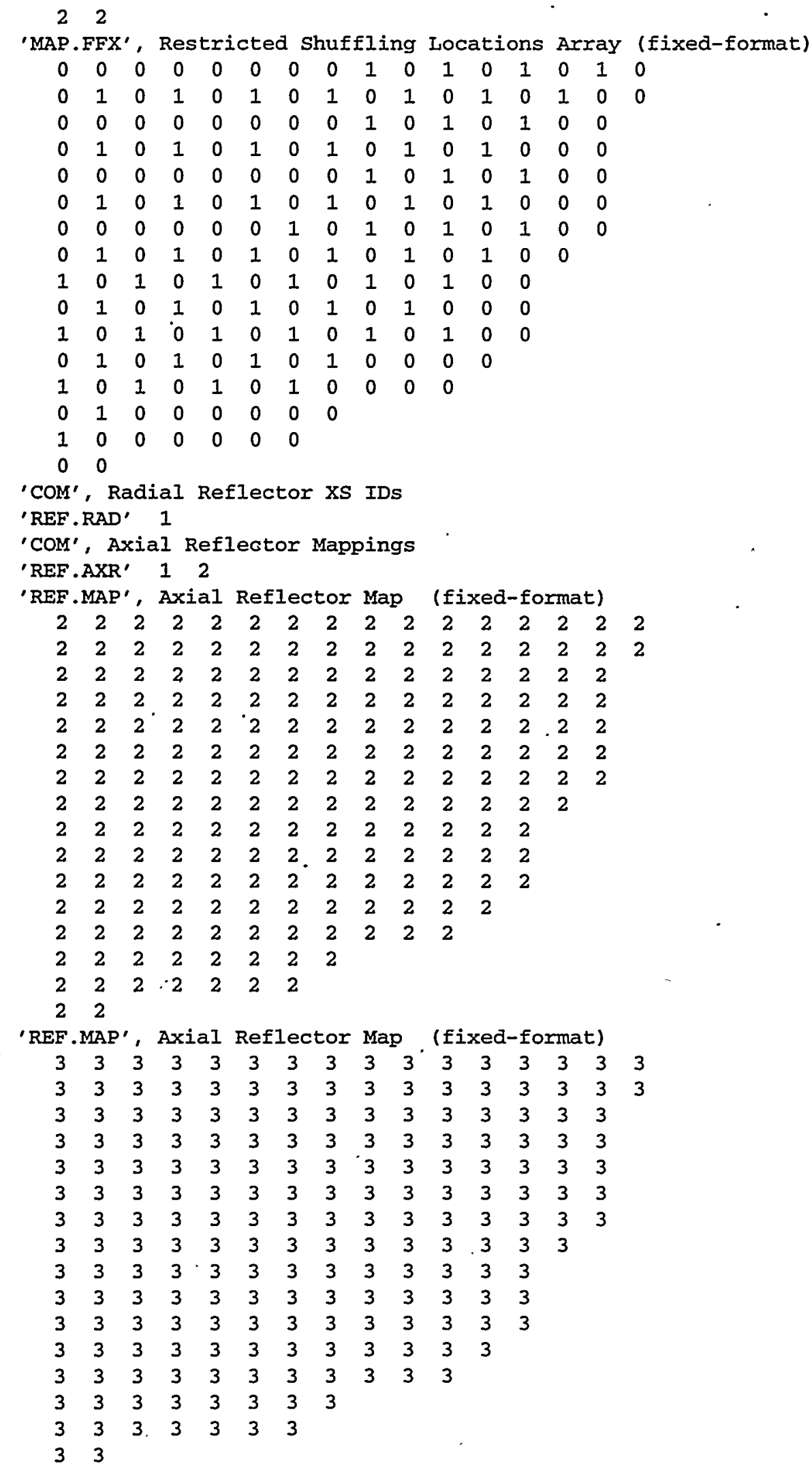




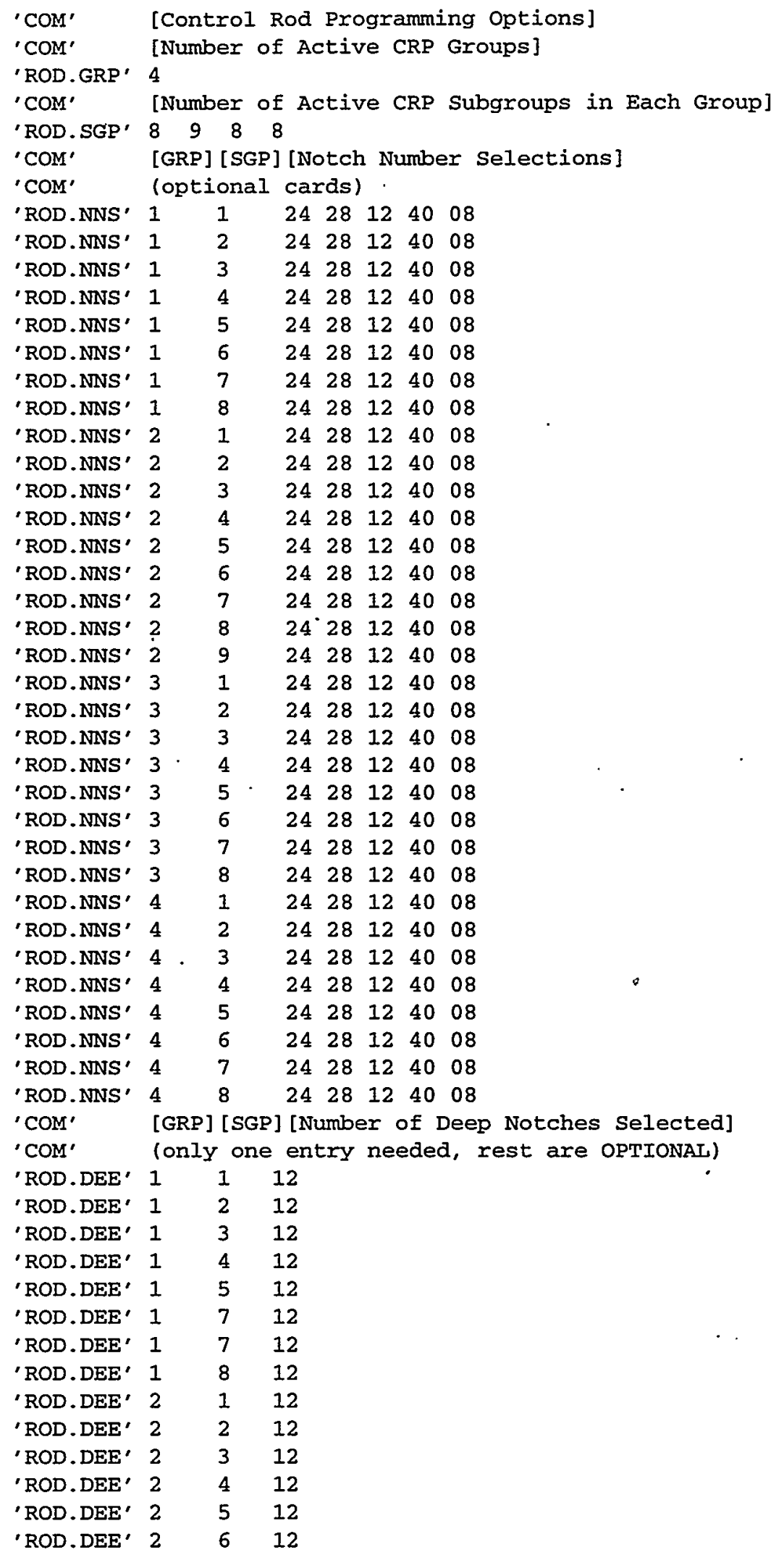




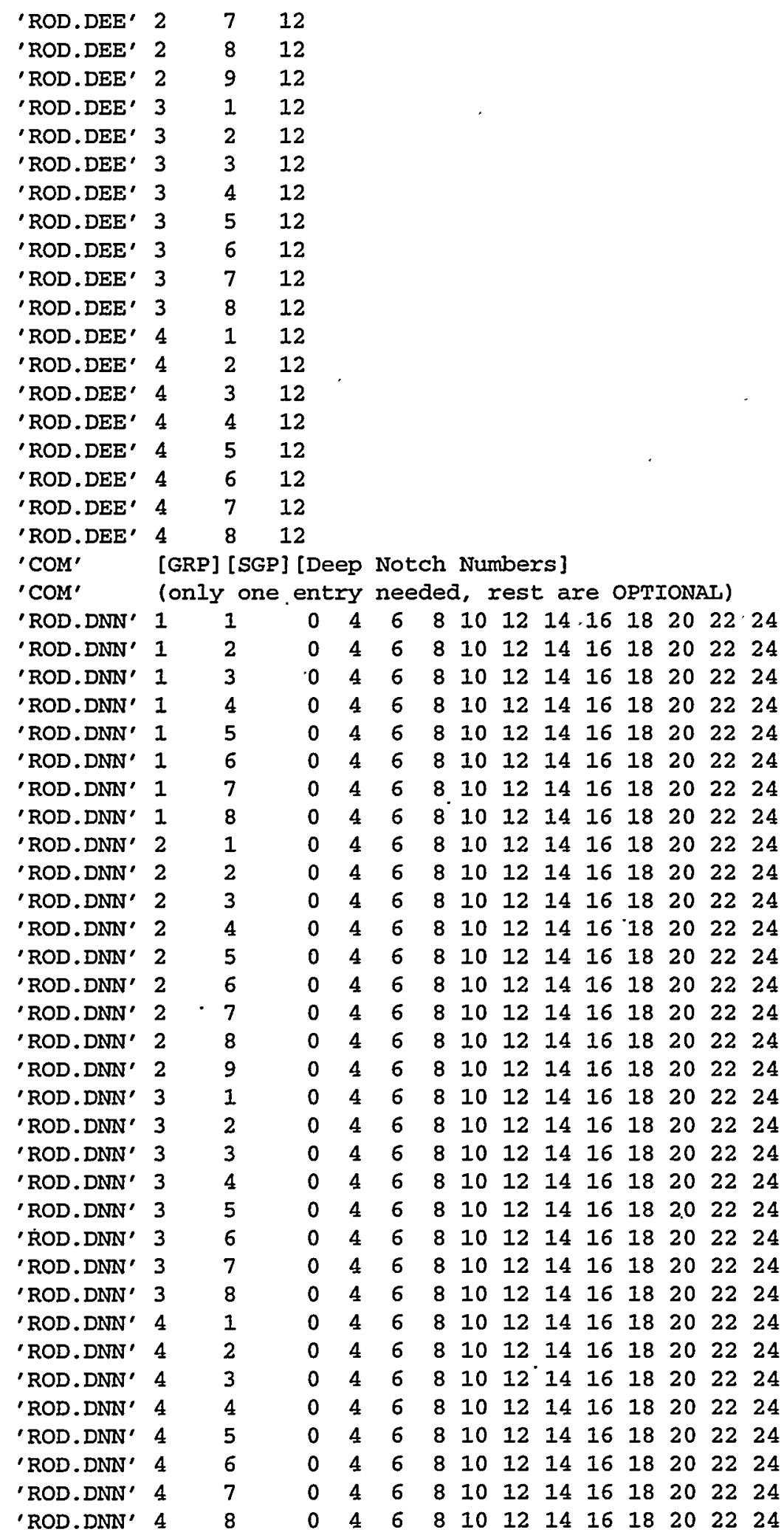

' COM' [GRP] [SGP] [Number of Shallow Notches Selected] ' COM' (only one entry needed, rest are OPTIONAL) 


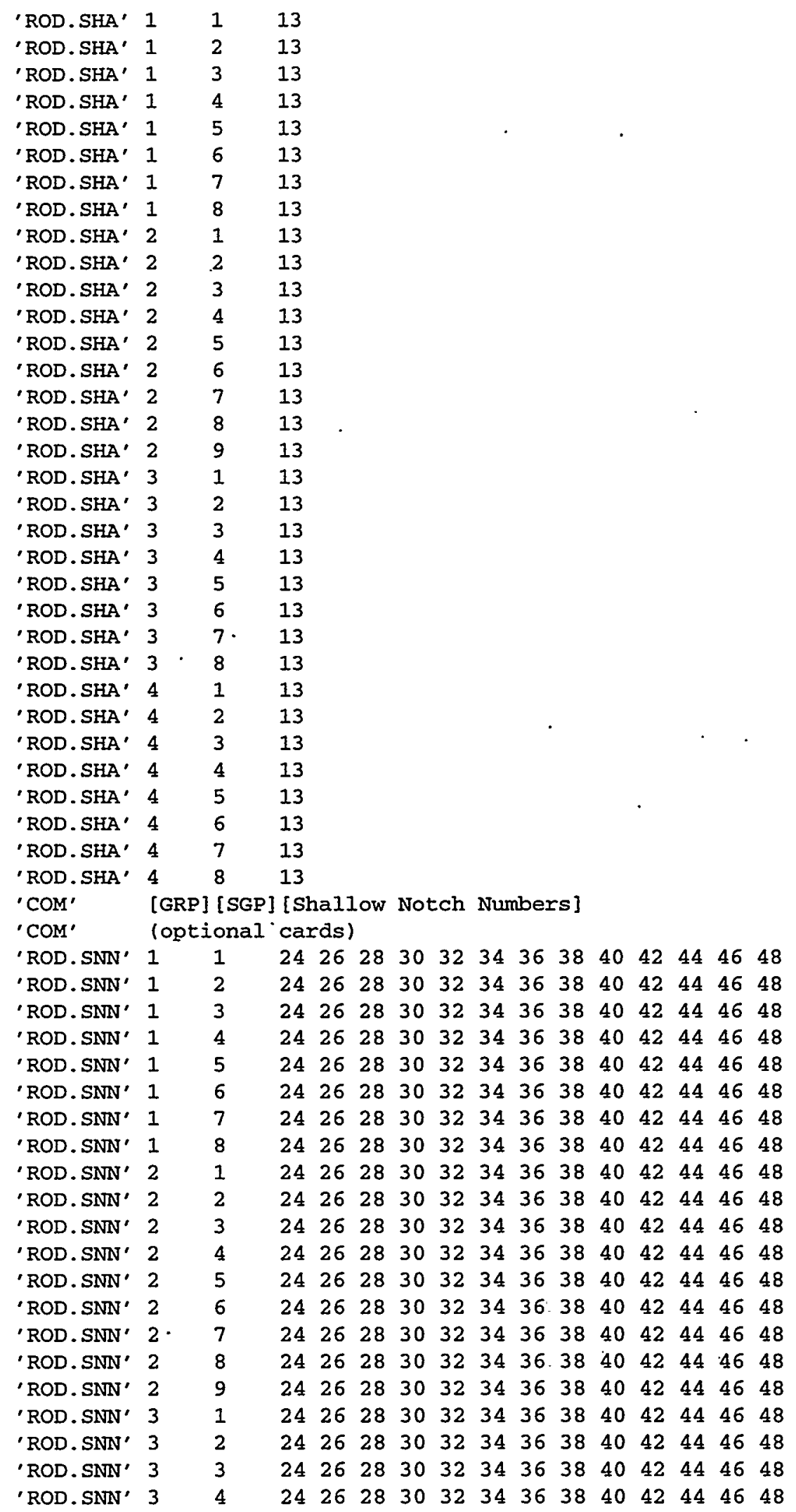




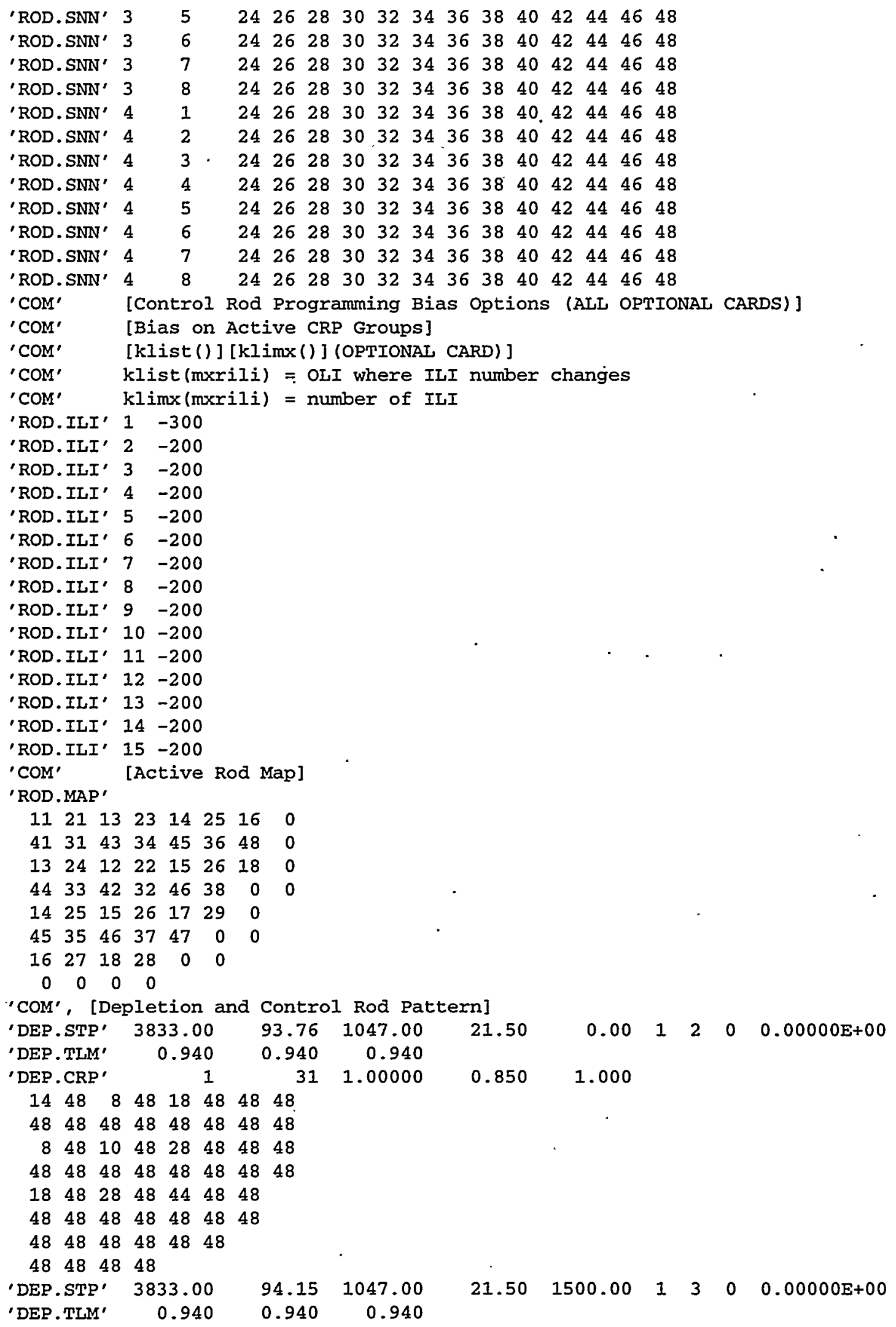




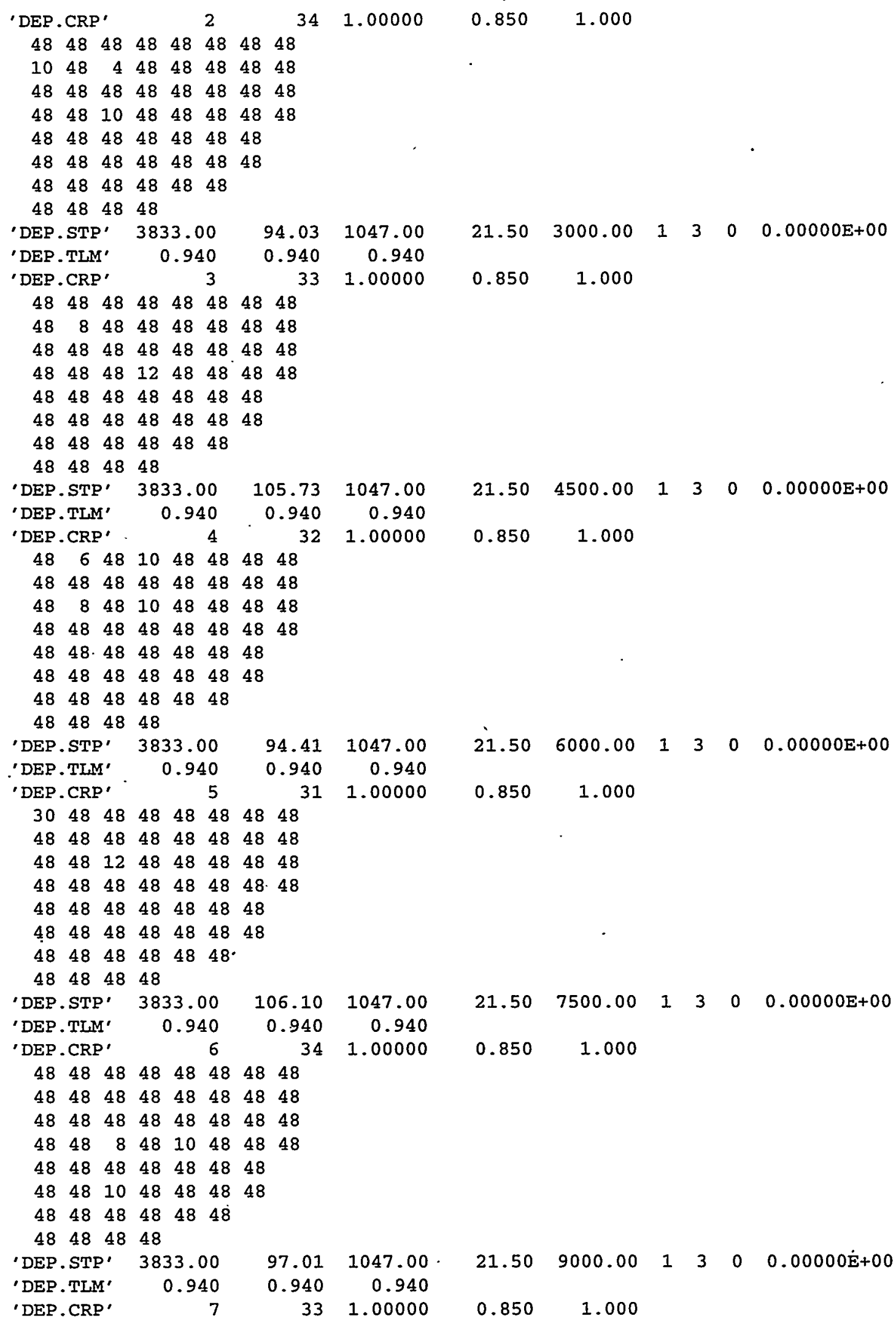




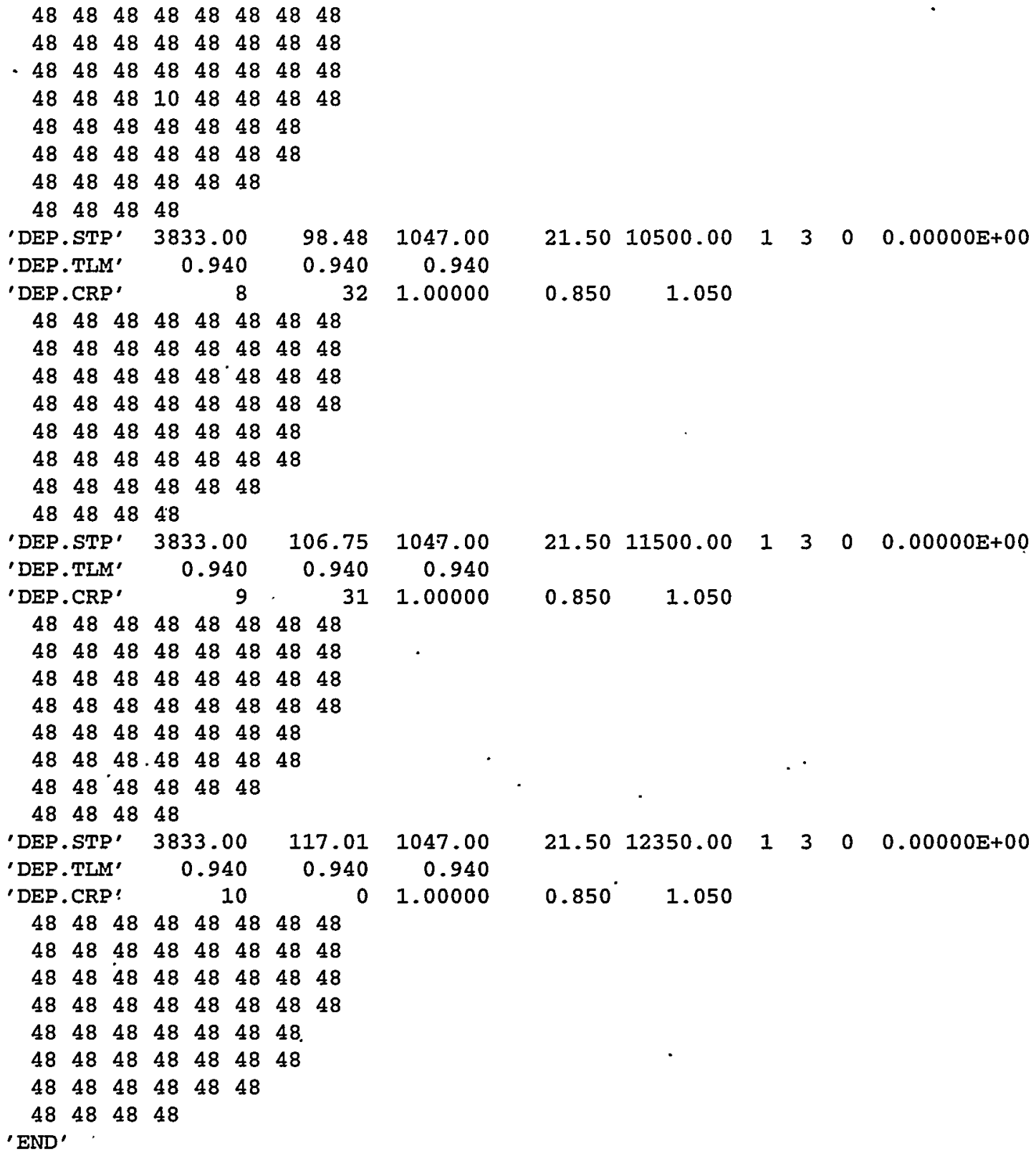




\section{A5. FORMOSA-B Sample Output File}

Since the complete FORMOSA-B output file is very long, only part of this file for the equilibrium cycle, full MOX core is shown below. The complete FORMOSA-B output is available in /u10/fmdp2/GEBWR.DIR.

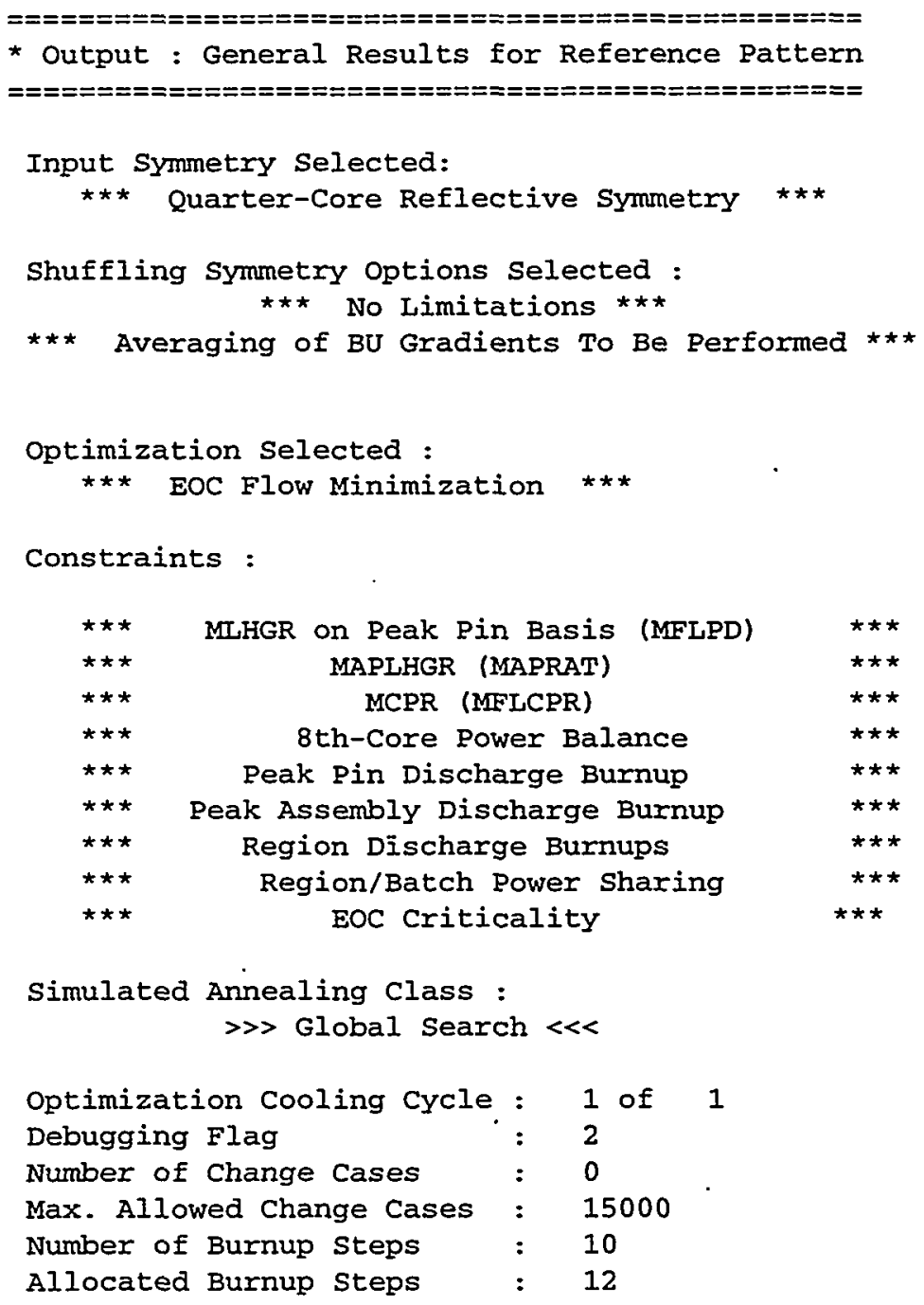


CRD Notches Inserted

Total Core Loading (MTU) Average Absolute Fast Flux Average Absolute Thermal Flux Fission Product Conditions Thermal Hydraulic Conditions
642

153.3893

$2.0964 \mathrm{E}+14$

$4.1605 \mathrm{E}+13$

: EqXe \& PkSm

: $\mathrm{T} / \mathrm{H}$, Flow on

Reference Control Rod Pattern

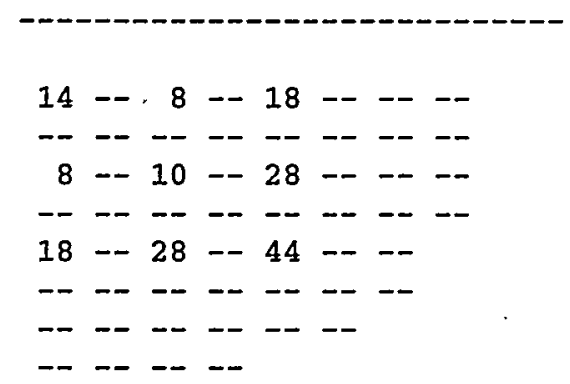

EDIT: IPROPT[ 1] $=2$

Assembly $\mathrm{k}$-infinity Map (Reference IP / WITH Converged Feedbacks)

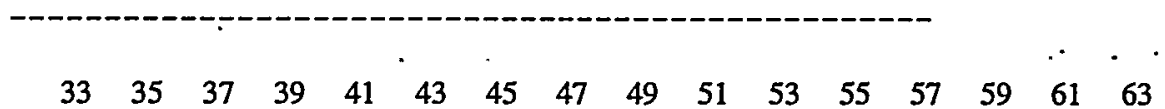

32- 0.89741 .07061 .09050 .93320 .93651 .08521 .09300 .89960 .91351 .00371 .01551 .00561 .01721 .10761 .02741 .0132 30- 1.08741 .00930 .99371 .01040 .98581 .00970 .99831 .01271 .00221 .01441 .01331 .01511 .03681 .02101 .11721 .0132 28- 1.09050 .99621 .06811 .08731 .09211 .08401 .09231 .08841 .01301 .09211 .01321 .09151 .01681 .06631 .0036 26- 0.93521 .01051 .08820 .87480 .87041 .01040 .99860 .94550 .93531 .01261 .01111 .01381 .04231 .10941 .0068 24- 0.93010 .98831 .09250 .87180 .94351 .08651 .09331 .02210 .94611 .09041 .01221 .09421 .01691 .07661 .0090 22- 1.07231 .01031 .08641 .01051 .08861 .01020 .99461 .01070 .99241 .01161 .02011 .01411 .05931 .11271 .0194 20- 1.09470 .99771 .09351 .00191 .09390 .98881 .01001 .08661 .01051 .07921 .01271 .09611 .01981 .11741 .0073 18- 0.90201 .01381 .05770 .94631 .00951 .01071 .08850 .99741 .00401 .01151 .02841 .01741 .11321 .0066 $16-0.91401 .00371 .01390 .93940 .94670 .99871 .01071 .00150 .99861 .09221 .01491 .08570 .9913$ 14- 1.00601 .01461 .09231 .01291 .09211 .01201 .05971 .01171 .09311 .01401 .07571 .10061 .0115 $12-1.01571 .01281 .01331 .01101 .01231 .02341 .01301 .03071 .01481 .07671 .02151 .09741 .0166$ $10-1.00811 .01521 .09351 .01371 .09441 .01391 .09711 .01691 .10101 .10011 .09571 .0232$ 08- 1.01731 .03241 .01671 .04181 .01641 .06091 .01941 .11220 .99991 .01321 .0211 $06-1.10771 .02101 .06661 .10941 .10521 .11221 .11630 .9994$ $04-1.02741 .11611 .00131 .00731 .01631 .02211 .0187$ $02-1.00881 .0140$

\section{EDIT: IPROPT[ 3] $=2$}

Bundle Average Exposure Map (GWD/STU) (NEM / Reference LP)

\section{$\begin{array}{llllllllllllllll}33 & 35 & 37 & 39 & 41 & 43 & 45 & 47 & 49 & 51 & 53 & 55 & 57 & 59 & 61 & 63\end{array}$}

32- 24.46814 .69114 .04115 .21913 .77615 .67413 .42529 .1460 .000028 .7350 .000028 .4380 .000015 .1380 .000029 .376 30- 15.2830 .000028 .5720 .000027 .9940 .000026 .9030 .000028 .0830 .000025 .9730 .000023 .5870 .000011 .42429 .499 28- 13.86028 .15113 .30015 .42513 .19915 .18913 .18415 .0960 .000015 .0190 .000014 .3840 .000020 .42328 .660 
26- 15.0020 .000015 .2540 .000028 .4220 .000028 .2620 .000027 .7640 .000026 .7020 .000022 .70814 .51929 .986 24- 13.54828 .59412 .99628 .20010 .80615 .64412 .83615 .2530 .000015 .2370 .000014 .6410 .000013 .88330 .082 22- 14.7270 .000014 .9080 .000015 .3100 .000027 .1520 .000027 .8830 .000025 .0430 .000020 .35614 .29828 .996 $20-13.44426 .97512 .96327 .86912 .67626 .2230 .000015 .2280 .000014 .3070 .000015 .3570 .000011 .52229 .560$ 18- 29.0750 .000013 .4760 .000014 .3510 .000014 .8600 .000023 .9670 .000024 .0940 .000012 .49730 .218 $16-0.000028 .0610 .000027 .6080 .000028 .4920 .000025 .5240 .000014 .6020 .000014 .88429 .933$ 14- 28.474 0.0000 15.055 0.0000 15.0120.000013.3360.000014.391 0.0000 17.804 13.474 29.714 $12-0.000026 .7200 .000026 .6980 .000024 .6190 .000023 .7500 .000017 .6230 .00009 .097929 .801$ 10- 28.0420 .000014 .3170 .000014 .5370 .000015 .2440 .000015 .57414 .1158 .966826 .753 $08-0.000024 .1640 .000022 .7130 .000020 .0240 .000012 .53929 .59929 .39928 .693$ $06-15.3640 .000020 .34014 .16515 .42514 .04011 .48129 .229$ $04-0.000011 .25428 .83229 .66828 .25328 .00629 .671$

02- 28.30229 .408

EDIT: IPROPT[ 4] = 1

Bundle Average Relative Power Fraction Map (NEM/ Reference LP)

$\begin{array}{llllllllllllllll}33 & 35 & 37 & 39 & 41 & 43 & 45 & 47 & 49 & 51 & 53 & 55 & 57 & 59 & 61 & 63\end{array}$

32- 1.02781 .30331 .32011 .06241 .06101 .29641 .28150 .92150 .91870 .98181 .04670 .95590 .97130 .92070 .68570 .3729 $30-1.33631 .27621 .17821 .23201 .14661 .25051 .15821 .16361 .04071 .09411 .02061 .04360 .94480 .85660 .69730 .3581$ 28- 1.33741 .18391 .29691 .28871 .29291 .30361 .31031 .24941 .15231 .18481 .11281 .11180 .98340 .82320 .5507 26- 1.05431 .23221 .29000 .99510 .94591 .23391 .16581 .10571 .00881 .14951 .06631 .08010 .95580 .84990 .5053 241.04001 .13941 .29140 .94641 .04851 .28381 .31121 .16751 .07621 .20721 .13691 .12540 .97570 .80600 .4701 $22-1.25081 .23481 .29691 .22991 .28401 .23341 .15981 .21701 .10511 .16521 .07391 .06180 .93800 .79940 .4429$ 20- 1.24881 .13621 .29511 .16131 .30731 .15501 .22811 .27761 .20021 .20351 .10821 .06780 .88590 .71410 .3878 18- 0.90661 .12961 .19371 .08901 .14921 .21751 .27921 .20181 .12281 .15021 .02960 .95420 .82310 .5017 16- 0.90721 .02471 .12311 .00001 .06361 .10481 .19981 .11121 .14651 .16331 .03000 .89900 .5895 $14-0.97521 .08561 .17471 .14131 .20281 .15201 .17631 .14751 .16521 .05750 .96500 .81670 .4852$ $12-1.04131 .01291 .10971 .06241 .13481 .07441 .09991 .03451 .03450 .96940 .83590 .67800 .3877$ $10-0.95461 .04051 .11311 .08151 .12941 .06571 .07400 .96770 .92540 .82220 .67860 .4405$ 08- 0.96940 .93840 .98590 .96340 .98640 .94990 .89490 .83600 .60900 .49280 .3945 $06-0.91850 .85690 .82830 .87360 .84790 .81490 .72840 .5077$ $04-0.68470 .69740 .55460 .51820 .50050 .46250 .4011$ 02- 0.36890 .3589

EDIT: IPROPT[ 4] $=\dot{1}$

Nodal Relative Power Fractions (NEM / Reference LP)

$\begin{array}{llllllllllllllll}33 & 35 & 37 & 39 & 41 & 43 & 45 & 47 & 49 & 51 & 53 & 55 & 57 & 59 & 61 & 63\end{array}$

260.53020 .46840 .44440 .45420 .42700 .38990 .36490 .39790 .32970 .31680 .26110 .25750 .20200 .17460 .13200 .1025 250.87520 .78640 .74180 .77470 .72080 .65400 .60560 .62360 .54190 .53250 .42880 .42650 .33630 .30660 .22740 .1706 241.17121 .15071 .09691 .14981 .06790 .97480 .89320 .82060 .76420 .71810 .60820 .57100 .48140 .44950 .32490 .2180 $231.33931 .35461 .32851 .36921 .30691 .17581 .10180 .95460 .92290 .83270 .7340 \quad 0.66360 .58700 .54560 .39960 .2527$ 221.40141 .40591 .37551 .35991 .31791 .23111 .19011 .04181 .00980 .89730 .79930 .71880 .64600 .60380 .44860 .2842 211.47701 .49461 .46751 .12091 .11051 .32961 .34101 .13961 .14360 .98220 .91220 .79050 .74300 .68790 .51370 .3046 201.47381 .46911 .44291 .06551 .07211 .35401 .41091 .20811 .23181 .04050 .98780 .84440 .81220 .75330 .56400 .3273 191.32931 .38271 .36801 .02381 .03851 .32861 .40371 .24491 .27091 .07441 .02230 .88150 .85040 .79600 .59870 .3536 181.02961 .34611 .34901 .02761 .04291 .35721 .42911 .31831 .35501 .14811 .10930 .96270 .93630 .86650 .66080 .3882 170.96271 .33351 .36241 .04601 .06961 .39701 .45851 .29021 .35461 .18791 .19771 .03541 .02980 .94450 .72400 .4111 160.93311 .29931 .33791 .03581 .06381 .38101 .42541 .00411 .04411 .17901 .22081 .07731 .07310 .98980 .76170 .4307 150.91331 .26681 .30721 .01991 .04751 .35201 .37520 .93290 .96921 .15961 .22011 .09721 .09381 .01860 .78940 .4497 140.92801 .30531 .35191 .05131 .08581 .39071 .41130 .92920 .98331 .16681 .27431 .13361 .16311 .08380 .83890 .4584 130.93241 .31561 .36301 .06101 .09541 .39521 .41160 .92300 .98131 .16361 .28871 .14931 .19511 .12090 .87040 .4685 
120.93311 .31111 .35611 .06211 .09091 .38051 .38890 .91490 .96691 .15261 .27741 .15231 .20211 .13880 .88900 .4792 110.95361 .35201 .39651 .10121 .11741 .40991 .40970 .92000 .96631 .14621 .29871 .16681 .24141 .18070 .91890 .4819 100.96871 .38161 .42481 .12561 .13421 .42831 .41500 .91190 .94701 .12951 .29411 .17221 .25611 .20500 .93070 .4810 90.98121 .39821 .44181 .13821 .14281 .43871 .41020 .90110 .91781 .10821 .27191 .17101 .25321 .21450 .93020 .4780 81.00961 .45231 .49581 .18021 .18071 .48521 .44490 .90220 .90691 .09741 .27971 .18601 .28341 .25440 .94730 .4766 71.04431 .53631 .57711 .24131 .23371 .56091 .50620 .90430 .89801 .08041 .30261 .20621 .33411 .31380 .96770 .4705 61.06961 .61641 .65111 .28851 .27071 .63451 .56330 .89890 .87811 .05081 .31011 .21541 .36901 .35610 .96910 .4590 51.06981 .66521 .69611 .29071 .26751 .68691 .59900 .87860 .83821 .00511 .28341 .20081 .35691 .34460 .93140 .4350 41.01631 .59331 .63571 .19371 .18681 .64431 .55050 .82150 .75660 .94271 .19621 .14511 .25511 .22990 .82180 .3928 30.84741 .22911 .29770 .89760 .93641 .32311 .25000 .69730 .61100 .83880 .98411 .00170 .99690 .93530 .62590 .3307 20.50610 .66870 .69390 .48240 .49700 .70640 .67710 .45810 .37910 .59430 .60410 .67070 .58370 .50290 .35730 .2185

$260.46590 .44320 .4668 \quad 0.41800 .44600 .3753 \quad 0.39100 .3343 \quad 0.3432 \quad 0.28260 .2768 \quad 0.22950 .22020 .16750 .13170 .0985$ 250.77940 .72070 .78170 .68280 .68690 .61120 .62630 .54460 .55490 .46360 .46990 .38000 .36850 .28450 .23140 .1785 241.14261 .02731 .06870 .97700 .91140 .87730 .84550 .77800 .74230 .66190 .64350 .54450 .50040 .41060 .33910 .2354 231.34841 .21961 .22131 .16211 .04571 .05340 .98870 .94400 .86670 .80360 .75220 .66260 .59610 .50480 .42450 .2739 221.40061 .27211 .25601 .19781 .09001 .11651 .05871 .02520 .94190 .87750 .81240 .72660 .65490 .56080 .48130 .3045 211.49701 .37601 .30331 .28051 .14201 .23211 .15541 .16351 .04271 .00440 .90060 .83550 .73560 .64570 .55790 .3251 201.50141 .38581 .28431 .27911 .15691 .27521 .20661 .24341 .11081 .08470 .96860 .91100 .79740 .70750 .61420 .3450 191.41781 .31561 .22271 .23001 .13791 .25661 .21311 .26081 .14631 .11781 .00550 .94860 .83760 .74360 .64900 .3653 181.38411 .32001 .23441 .25861 .17971 .30971 .27351 .33471 .22551 .20611 .08901 .03920 .91910 .81820 .69010 .3946 171.37351 .34271 .24711 .30331 .21611 .37331 .31701 .39491 .27211 .29121 .16401 .13760 .99910 .89860 .74140 .4131 161.33851 .31661 .22681 .29351 .21071 .36901 .30501 .37821 .26351 .30471 .18951 .17701 .03930 .94000 .77810 .4282 151.30491 .28431 .20641 .27021 .19701 .34391 .28061 .33941 .24151 .29221 .19621 .19091 .06170 .96440 .80570 .4423 141.34511 .33341 .22551 .32081 .22371 .39621 .30011 .37831 .25411 .34271 .23031 .25991 .11321 .02720 .85370 .4464 131.35621 .34671 .22911 .33371 .22881 .40361 .29461 .37591 .24681 .34951 .23771 .28601 .13821 .06070 .88360 .4511 121.35151 .33861 .22591 .32421 .22171 .38241 .27151 .34341 .22321 .32591 .22711 .28311 .14491 .07440 .90150 .4555 111.39381 .38181 .24551 .36281 .24321 .40931 .27181 .34451 .20481 .33491 .23231 .31621 .17141 .11290 .92860 .4514 101.42461 .40391 .25961 .38031 .25901 .41601 .26731 .32621 .17871 .31891 .22531 .32321 .18521 .12920 .93750 .4445 91.44381 .40951 .26911 .38261 .27061 .40971 .26261 .29881 .15151 .28891 .21021 .31221 .18981 .13050 .92940 .4351 81.49841 .45581 .29751 .42521 .30911 .44881 .28701 .31161 .14451 .29351 .21331 .33621 .21661 .16000 .94550 .4270 71.58131 .53121 .33301 .49601 .36771 .52261 .33211 .34801 .14161 .31601 .22411 .38261 .25991 .20290 .96460 .4148 61.66021 .59691 .35781 .55541 .42131 .59201 .37661 .37391 .12691 .32391 .22461 .41551 .29421 .22730 .96460 .3993 51.71611 .62231 .35371 .56921 .44371 .62471 .40101 .36251 .08961 .29591 .20371 .40581 .29571 .20350 .92530 .3756 41.66571 .53481 .28871 .47041 .38361 .54241 .35621 .26911 .01791 .20271 .14681 .30971 .23231 .09620 .80960 .3442 31.31211 .21841 .10711 .15861 .15671 .22081 .15171 .01670 .88310 .97851 .00381 .05271 .03670 .85430 .60400 .2976 20.70500 .70670 .74340 .66830 .71670 .69990 .72150 .60000 .60300 .59060 .66780 .62410 .61090 .49060 .34050 .2054

$260.44590 .46780 .44040 .41730 .39620 .37890 .34990 .32900 .2998 \quad 0.27860 .24900 .23020 .19720 .17280 .1466$ 250.74260 .78230 .74190 .69730 .65840 .63530 .58120 .54910 .49060 .46730 .41110 .39390 .33230 .30840 .2432 241.09561 .06791 .05741 .03150 .96960 .93470 .86090 .81690 .70550 .69530 .59380 .58740 .48280 .45350 .3187 231.32441 .21911 .23471 .23821 .18411 .11641 .06151 .00900 .86240 .86260 .72790 .71740 .59390 .54360 .3759 221.36961 .25301 .28451 .29171 .24851 .18161 .14911 .09580 .94440 .94510 .80010 .78540 .65650 .59500 .4213 211.46591 .30301 .37651 .37501 .35741 .29171 .30281 .24531 .08301 .08460 .92340 .89370 .75700 .66490 .4567 201.46031 .28781 .39731 .37331 .37201 .33271 .37581 .32801 .17121 .17151 .00480 .96860 .82740 .71540 .4906 191.38911 .22751 .33661 .31391 .32031 .31521 .37461 .34841 .20341 .20891 .04291 .00810 .86420 .74610 .5223 181.37011 .24031 .33451 .32191 .33081 .35761 .41981 .41441 .29911 .28891 .14031 .09080 .94910 .80760 .5685 171.38501 .25451 .36421 .35621 .37521 .41701 .48981 .49391 .39931 .38181 .24641 .18571 .04230 .86940 .6008 161.36081 .23421 .35241 .34871 .37361 .42141 .49801 .50761 .42231 .41331 .28601 .23581 .08440 .90300 .6248 151.32991 .21341 .32861 .32901 .35481 .40531 .48051 .49681 .41741 .41571 .29531 .25461 .10480 .92400 .6461

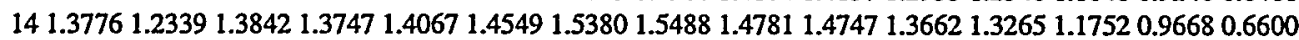
131.39031 .23811 .40061 .38561 .41561 .45961 .53751 .54281 .47781 .47961 .38551 .35421 .20700 .98920 .6719 121.38341 .23451 .39271 .37631 .39901 .43691 .49441 .48461 .42211 .44421 .36841 .35171 .21260 .99880 .6822 111.42531 .25511 .43561 .40831 .42721 .46551 .49061 .45151 .39431 .43531 .38931 .38571 .25161 .02300 .6877 101.45321 .26941 .45271 .42691 .44021 .47151 .47321 .40691 .34491 .40991 .38361 .39871 .26671 .03650 .6893 91.46771 .27811 .44061 .43511 .44181 .46441 .45311 .37621 .30211 .38321 .36321 .39391 .26521 .04380 .6889 81.52131 .30701 .48471 .47701 .48591 .50561 .48471 .38781 .30631 .39271 .38331 .42861 .29741 .06720 .6941 71.60261 .34331 .56181 .54391 .56381 .58691 .55441 .42241 .33441 .42201 .43011 .49411 .35121 .09680 .6960 61.67601 .36801 .62601 .60201 .63851 .66871 .62411 .44861 .34981 .44061 .46621 .55171 .39051 .11510 .6901 51.72041 .36341 .63851 .62521 .67921 .71831 .66501 .44361 .32601 .42791 .46101 .56181 .38371 .10590 .6692 41.66001 .29831 .52161 .55151 .59571 .63561 .59141 .36941 .22411 .35251 .36481 .44961 .28411 .04700 .6255 31.31661 .11371 .17191 .24501 .22201 .25071 .23331 .11240 .97491 .11981 .09421 .11701 .01800 .87950 .5394 
260.46240 .42230 .41870 .41730 .43890 .36060 .37070 .32160 .33230 .26760 .26650 .21740 .20190 .16390 .1369 250.78730 .68880 .69910 .68950 .73320 .59060 .60540 .52900 .53340 .44030 .44250 .36320 .35800 .28910 .2408 241.16240 .98261 .03320 .98540 .99170 .85110 .82450 .76430 .71940 .63680 .60610 .52990 .52410 .43600 .3196 231.37711 .16571 .24001 .18501 .15121 .03120 .96740 .93630 .84960 .78270 .71910 .65480 .62980 .54810 .3745 221.36161 .20431 .29431 .25671 .21981 .10271 .04001 .02120 .93330 .86220 .78780 .72430 .68880 .61060 .4165 211.11031 .28501 .37931 .30091 .22281 .21681 .13841 .16941 .04270 .99730 .88420 .83980 .77260 .69580 .4447 201.05611 .28081 .37731 .02660 .96761 .25541 .19081 .26121 .12331 .08820 .95510 .91960 .83130 .74540 .4695 191.01691 .23191 .31770 .94770 .90231 .23311 .20511 .29341 .17221 .12940 .99840 .95900 .86420 .77300 .4950

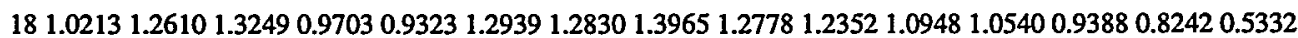
171.04061 .30651 .35981 .00860 .96341 .37341 .35501 .51041 .37321 .35171 .18601 .16001 .01510 .88460 .5579 161.03171 .29761 .35261 .01100 .97081 .38891 .37601 .55271 .41841 .39661 .22681 .20621 .05240 .92090 .5782 151.01721 .27541 .33331 .00310 .97341 .38021 .37931 .56671 .44571 .40851 .24531 .22441 .07290 .94470 .5977 141.05031 .32731 .38031 .04260 .99331 .44191 .41551 .64511 .49331 .48411 .29361 .30191 .12280 .99560 .6056 131.06161 .34151 .39181 .05330 .99761 .44911 .40951 .65441 .49711 .49391 .30541 .33151 .14541 .02360 .6143 121.06411 .33301 .38281 .04770 .99331 .41861 .36241 .53921 .39881 .44451 .29031 .32731 .14971 .03820 .6244 111.10331 .37181 .41491 .07040 .99671 .42791 .32741 .17271 .07991 .42471 .29141 .36261 .17401 .06990 .6257 101.12411 .38781 .43281 .07320 .99261 .41601 .28791 .06840 .97951 .38221 .28361 .37161 .18731 .08950 .6251 91.13161 .38761 .43971 .06510 .98901 .39501 .26561 .02770 .94541 .34561 .27341 .36421 .19281 .10140 .6244 81.17071 .42771 .48031 .08781 .00211 .42291 .27981 .02930 .93731 .35801 .29181 .39711 .21921 .13280 .6245 71.22781 .49541 .54601 .12361 .01821 .48551 .31541 .04650 .93351 .39811 .32991 .45831 .25841 .16950 .6162 61.26831 .54991 .60111 .14281 .02241 .54121 .34701 .05070 .91921 .42771 .36281 .50911 .28791 .18850 .6003 51.25791 .55451 .61881 .11750 .99661 .55181 .34941 .01860 .88381 .41571 .36981 .51591 .28921 .17010 .5749 41.14731 .44451 .53861 .01430 .92041 .44521 .27970 .92450 .80941 .31321 .31361 .42061 .23251 .08910 .5364 30.85071 .13051 .22870 .78310 .76121 .12711 .07490 .72000 .67481 .04371 .12281 .13221 .04700 .86820 .4705 20.45620 .65090 .66280 .45320 .49750 .64610 .69420 .42180 .44570 .60970 .71690 .65850 .63870 .47580 .3269

260.45570 .45380 .39860 .43900 .38500 .35590 .32860 .31560 .28840 .26530 .23420 .21260 .18320 .15870 .1284 $250.78180 .70050 .66170 .73530 .64630 .59910 .54800 .53160 .47600 .4487 \quad 0.38870 .36400 .31250 .28510 .2234$ $241.11170 .92680 .97230 .99610 .94890 .89250 .81580 .79630 .68860 .6727 \quad 0.56650 .55080 .46030 .42760 .2949$ 231.29871 .05851 .18611 .15691 .17111 .08131 .01570 .98790 .84650 .82880 .70010 .69430 .57320 .52470 .3459 221.29351 .09871 .25571 .22511 .26701 .15541 .10791 .08050 .92940 .90940 .77450 .77050 .63850 .58280 .3867 211.07651 .14561 .36501 .22881 .35001 .26401 .26691 .23341 .07331 .04130 .90010 .89350 .73940 .65610 .4127 201.03781 .15351 .37570 .97241 .08821 .30151 .34711 .32521 .17101 .13010 .98680 .97520 .80930 .69880 .4330 191.01121 .13411 .32270 .90671 .01511 .28561 .35871 .36251 .21751 .17481 .02901 .01610 .84480 .72940 .4559 181.02251 .17741 .33230 .93611 .02641 .33831 .42051 .45301 .33361 .27031 .13221 .09540 .92700 .77750 .4917 171.05021 .21391 .37720 .96721 .07391 .41401 .51481 .56271 .46211 .38081 .24791 .19261 .01820 .83400 .5145 161.04711 .2101 i.3765 0.97491 .09051 .43761 .54901 .61461 .52031 .43191 .29741 .24011 .06160 .86970 .5340 151.03471 .19891 .35890 .97781 .09021 .43761 .55211 .63931 .54771 .45191 .31521 .26131 .08390 .89400 .5534 141.07661 .22551 .41270 .99841 .14671 .49701 .62911 .71851 .63591 .52611 .39681 .33651 .15580 .94540 .5613 131.09151 .23201 .42291 .00311 .16041 .50371 .63281 .73011 .65321 .53791 .42161 .36611 .19000 .97520 .5707 121.09101 .22671 .40700 .99911 .14651 .47331 .57101 .61231 .54401 .49311 .40491 .36461 .19830 .99160 .5820

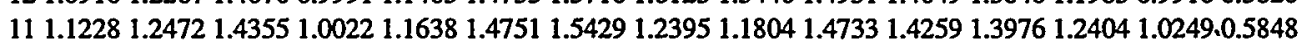
101.13441 .26021 .44730 .99751 .15321 .46411 .49711 .13401 .07781 .43991 .42281 .41181 .26011 .04340 .5854 91.12421 .26761 .44590 .99241 .12581 .44891 .46031 .10181 .03781 .41731 .40661 .41471 .26441 .04890 .5857 81.15771 .30111 .48781 .00311 .15211 .47831 .48391 .10881 .03941 .44071 .43651 .45291 .30391 .08170 .5870 71.21091 .35251 .56301 .01511 .20201 .54401 .54601 .13131 .05571 .49521 .49891 .51641 .36621 .12320 .5803 61.24431 .39621 .63251 .01401 .23651 .60821 .60461 .13961 .05711 .54491 .55261 .57091 .41391 .14590 .5660 51.21751 .40281 .66120 .98201 .21941 .63721 .62741 .10981 .01921 .55771 .56431 .59231 .41331 .12320 .5403 41.08201 .32411 .56030 .90251 .09761 .55081 .53531 .01760 .91861 .46741 .47101 .52691 .31231 .01820 .4999 30.79161 .09571 .18210 .74540 .80771 .20391 .17970 .80340 .71251 .14821 .16931 .24071 .03200 .76460 .4364 20.43430 .68280 .64470 .48860 .44740 .64770 .64480 .43880 .41880 .63240 .67970 .67700 .59020 .42480 .2973

260.40050 .38120 .38150 .36170 .35580 .32860 .33950 .28980 .29780 .24790 .24030 .19890 .17940 .14260 .1158 $\begin{array}{llllllllllllllll}25 & 0.6728 & 0.6202 & 0.6391 & 0.5923 & 0.5986 & 0.5379 & 0.5550 & 0.4753 & 0.4892 & 0.4097 & 0.4160 & 0.3351 & 0.3202 & 0.2541 & 0.1977\end{array}$ 240.99680 .88670 .93920 .85280 .89240 .77800 .75500 .68910 .66710 .59560 .58930 .49260 .47980 .38560 .2598 231.19141 .06221 .12101 .03221 .08310 .94930 .88660 .84670 .78820 .73400 .70330 .61270 .58600 .48930 .3060 221.23831 .12021 .18481 .10281 .15781 .02550 .95730 .92900 .86100 .81000 .76820 .68160 .64590 .55110 .3450 211.32261 .22501 .29271 .21591 .26801 .14761 .05611 .07070 .96240 .94050 .86450 .79360 .73100 .63200 .3717 201.31611 .26361 .33251 .25381 .30651 .20171 .12081 .15691 .03741 .02640 .93250 .87210 .79000 .68620 .3989 191.28671 .24341 .31391 .23091 .29051 .20641 .14931 .19231 .08231 .06980 .97240 .91230 .82160 .71740 .4253 
181.30961 .29541 .35441 .29111 .34191 .28871 .24041 .29961 .18621 .17821 .06601 .00560 .89100 .76470 .4596 171.34681 .35831 .41391 .37041 .41771 .38911 .32921 .42221 .28661 .30041 .15951 .11030 .96450 .81970 .4818 161.33301 .35551 .41931 .38651 .44131 .42071 .36361 .47051 .33451 .35311 .20281 .15881 .00090 .85520 .5012 151.30721 .33301 .40431 .37891 .44121 .42241 .37531 .48401 .35931 .37241 .22391 .18121 .02130 .88120 .5207 . 141.34711 .38651 .45651 .44181 .50231 .49501 .42161 .56771 .41221 .45801 .27961 .26221 .07510 .93150 .5309 131.35391 .39611 .46301 .45041 .50981 .50471 .42281 .57861 .41871 .48061 .29851 .29751 .10170 .96230 .5419 121.34181 .37721 .44151 .42101 .47921 .46871 .38671 .52371 .37931 .45371 .29111 .30051 .10990 .98170 .5544 111.37091 .40421 .47001 .43091 .48151 .47341 .36711 .50241 .34881 .46541 .30621 .34341 .14301 .01670 .5594 101.38611 .40941 .47431 .41841 .46941 .45251 .33901 .45681 .30941 .45091 .30971 .36121 .16721 .04050 .5621 91.38991 .40051 .46301 .39591 .45191 .42251 .31611 .41841 .28381 .42801 .30921 .36301 .18611 .05740 .5647 81.43211 .43641 .50101 .42191 .47971 .44451 .32821 .43481 .29251 .45551 .33851 .40621 .23081 .09530 .5687 71.50351 .50541 .57871 .48141 .54261 .50341 .36311 .48371 .31741 .51701 .38961 .47951 .29571 .13980 .5654 61.56681 .56561 .65341 .53181 .60181 .55591 .39331 .52081 .33101 .56751 .43441 .54251 .35281 .16520 .5535 51.58851 .57781 .68751 .53331 .62381 .56431 .39391 .50851 .30521 .56801 .44841 .55841 .37461 .14560 .5285 41.49551 .46931 .58661 .41761 .53391 .45851 .32111 .38971 .20951 .45351 .38861 .46171 .31271 .04660 .4813 31.15611 .14371 .19981 .10291 .19091 .14091 .10851 .08731 .00671 .13721 .17341 .15351 .07780 .79630 .4072 20.61700 .65230 .65100 .62990 .63870 .65500 .70530 .62730 .66020 .65750 .74240 .66030 .59020 .42740 .2718

260.36990 .39530 .35250 .37200 .32900 .34020 .29120 .27790 .25470 .24400 .21240 .19240 .15690 .12540 .1037 250.61360 .63320 .58530 .60810 .54870 .55470 .47620 .46620 .41920 .41790 .35530 .33530 .26940 .22240 .1824 $240.90080 .85050 .86440 .82740 .81630 .75350 .68950 .69940 .60900 .61360 .5200 \quad 0.50560 .39660 .33210 .2394$ 231.10580 .98651 .06500 .96891 .01580 .88420 .84600 .86680 .75010 .74390 .64400 .62450 .49650 .42380 .2817 221.18981 .05151 .14891 .04051 .10730 .95360 .92510 .94940 .82720 .81750 .71440 .69240 .55850 .48540 .3185 211.33111 .14191 .29281 .13911 .26571 .05041 .05641 .08970 .96060 .94090 .83170 .79490 .64950 .56600 .3404 201.38051 .18731 .36211 .19001 .34511 .11741 .13411 .17631 .04851 .03110 .91050 .86940 .71480 .61960 .3633 191.36881 .19171 .35991 .20371 .35581 .14621 .16581 .21551 .09201 .08250 .95300 .91230 .75050 .65120 .3866 181.39251 .24971 .40341 .28001 .41631 .23531 .26951 .30941 .20261 .17761 .05300 .99320 .82490 .68910 .4161 171.42041 .29241 .47271 .35181 .51011 .32331 .38851 .42231 .32781 .28981 .16571 .08590 .90660 .73810 .4331 161.38931 .28231 .48281 .37341 .54471 .35721 .43361 .47181 .38131 .34491 .21771 .13610 .94940 .77320 .4494 151.34291 .26091 .46801 .37831 .54861 .36901 .44411 .48851 .40021 .36831 .24111 .16450 .97570 .80020 .4669 141.37961 .28221 .52791 .41611 .62711 .41631 .52601 .56751 .48931 .45631 .32741 .24061 .04450 .85130 .4721 131.38221 .27921 .53031 .41231 .63211 .41811 .54171 .58571 .51581 .48861 .36361 .27901 .08240 .88350 .4800 121.36291 .25871 .49011 .36681 .57201 .38191 .50881 .56021 .49631 .48011 .36401 .29091 .09900 .90360 .4897 111.38391 .26011 .48761 .33271 .54591 .36371 .51801 .56921 .51851 .51361 .40701 .33651 .14430 .93660 .4901 101.38831 .25471 .46931 .29311 .49971 .33641 .49941 .56181 .51381 .52111 .42261 .36511 .16880 .95260 .4890 91.38241 .24851 .44581 .26881 .46051 .31361 .46951 .54741 .49501 .50771 .42101 .38131 .17900 .95440 .4872 81.41441 .27051 .47531 .28101 .48271 .32581 .49161 .57821 .52711 .54901 .46411 .43401 .22210 .98480 .4859 71.46941 .31201 .54301 .31281 .54331 .36061 .54981 .64271 .59531 .63061 .54041 .51751 .28461 .02010 .4770 61.51411 .34771 .60601 .33811 .59851 .39141 .59831 .69911 .65101 .70401 .60521 .59251 .32801 .03440 .4620 51.52381 .35121 .62681 .32901 .61361 .39011 .59771 .71161 .64661 .72311 .61771 .62021 .31501 .00410 .4358 41.44371 .27971 .52261 .24601 .51281 .31431 .47761 .59681 .49941 .59371 .50601 .52311 .19650 .88710 .3926 31.15041 .06831 .16101 .03711 .16051 .09601 .14851 .23501 .14321 .19431 .17501 .17430 .91720 .65300 .3307 $20.61930 .66810 .63320 .66440 .63130 .6823 \quad 0.65600 .65140 .64000 .65250 .67320 .63420 .51560 .35960 .2219$

260.39890 .33660 .33910 .32360 .31680 .28980 .27770 .25640 .26010 .21930 .21100 .17160 .14270 .1242 250.62490 .54720 .57310 .53240 .53460 .47570 .46600 .42190 .43630 .36500 .36960 .29180 .24950 .2160 $240.81900 .77720 .82900 .76720 .80070 .68970 .69930 .61370 .60100 .53380 .5295 \cdot 0.42890 .37390 .2875$ 230.94840 .93840 .98780 .93750 .99090 .84730 .86760 .75700 .71210 .66120 .63590 .53520 .47710 .3416 221.03231 .01611 .06551 .02541 .08360 .92920 .95020 .83510 .77800 .73330 .69790 .59920 .54340 .3873 211.12671 .14671 .19511 .16871 .23141 .07101 .09180 .97050 .87930 .85620 .78810 .69670 .63460 .4193 201.19401 .21021 .25931 .24431 .30431 .16161 .18141 .05980 .96120 .94380 .85240 .76090 .69420 .4489 191.23051 .21991 .28251 .27321 .33751 .19661 .22161 .10181 .01770 .98870 .89120 .79850 .72930 .4777 181.30201 .29051 .34411 .37411 .42071 .30331 .31451 .21251 .12161 .09370 .97870 .88150 .77850 .5175 171.27381 .34851 .41991 .48551 .52661 .42571 .42791 .33861 .22621 .21261 .06770 .97360 .84090 .5439 160.99031 .33411 .43641 .52851 .57771 .47421 .47761 .39251 .27511 .26711 .11201 .02040 .88170 .5665 150.92211 .29951 .42981 .54511 .60201 .48831 .49381 .41161 .29811 .29111 .13691 .04710 .91020 .5896 140.91971 .33931 .48791 .62401 .68211 .57281 .57461 .50311 .36531 .38271 .19711 .12310 .97090 .6011 130.91561 .34031 .48911 .63631 .69411 .58451 .59311 .53331 .39091 .42171 .22631 .16311 .00680 .6144 120.90971 .31231 .43741 .52621 .58011 .53061 .56701 .51931 .38731 .42281 .23401 .17791 .02720 .6291 $110.91481 .31511 .41341 .16141 .23301 .50971 .57661 .55211 .41411 .47021 .26711 .22681 .06700 .6347^{\circ}$ 100.90601 .29721 .36671 .05741 .12781 .46381 .56821 .55511 .42771 .48891 .28811 .25311 .08850 .6378 90.89341 .26911 .31801 .01511 .09071 .42361 .55141 .54201 .43441 .48891 .30221 .26371 .09730 .6403 
80.89111 .28031 .33021 .01381 .09651 .43801 .58021 .58021 .47601 .53611 .34461 .31031 .13710 .6471 70.88721 .31371 .37341 .02721 .11871 .48421 .64361 .65561 .54631 .61931 .40731 .38031 .18710 .6473 60.87421 .33211 .40311 .02551 .12481 .51741 .69861 .71741 .60771 .68961 .46111 .43231 .21480 .6372 50.84281 .30391 .37620 .98611 .09051 .49961 .70741 .70801 .61361 .69711 .47641 .42661 .19140 .6094 40.77061 .18891 .23480 .88000 .98951 .37491 .58751 .48311 .43701 .55011 .40331 .30821 .08080 .5549 30.64870 .93520 .92860 .67350 .75981 .07061 .21920 .87120 .91961 .17141 .16011 .01090 .81180 .4631 20.42800 .54810 .52320 .39320 .41480 .61620 .64360 .45280 .48270 .64980 .70110 .57240 .44010 .3070

260.32780 .34530 .30040 .33250 .28870 .29700 .25470 .25850 .22190 .20590 .18050 .15980 .1441 250.53680 .52690 .49060 .53430 .47690 .49150 .42000 .44050 .36880 .35310 .30620 .28160 .2395

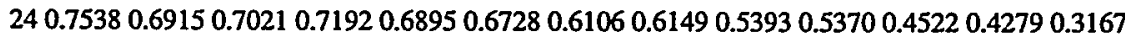
230.90810 .80590 .85510 .84740 .84780 .79650 .75220 .73080 .66920 .67470 .56550 .53480 .3758 220.99260 .88460 .93460 .92980 .93500 .87030 .82930 .79820 .74360 .74970 .63250 .59830 .4254 211.12270 .99151 .06711 .03551 .07630 .97130 .96370 .90070 .86890 .87090 .73890 .68420 .4639 201.20861 .06251 .14101 .11461 .15951 .04381 .05570 .97560 .95500 .95340 .81530 .73590 .4985 191.24721 .10101 .16591 .16201 .20311 .08821 .09931 .02060 .99940 .99790 .85620 .77350 .5321 181.33051 .18331 .25841 .26641 .31731 .19251 .20961 .12321 .10571 .08520 .94670 .83490 .5825 171.33121 .23401 .35631 .36141 .44381 .29281 .33471 .22581 .22621 .18951 .04840 .90610 .6194 161.02961 .23051 .38111 .40781 .50221 .34151 .38851 .27361 .28191 .24301 .09890 .94980 .6476 150.95781 .21371 .37951 .43761 .53111 .36811 .40811 .29761 .30681 .27041 .12640 .97980 .6742 140.97351 .23391 .44111 .48691 .61941 .42121 .49801 .36021 .40061 .35641 .21091 .04370 .6944 130.97361 .23341 .44421 .49351 .63861 .42911 .52521 .38441 .44161 .39791 .25471 .08180 .7132 120.96131 .21431 .39311 .39591 .53281 .39131 .50621 .38131 .44531 .40801 .26941 .10350 .7308 110.96181 .20351 .36721 .07671 .16971 .36031 .52801 .40371 .49611 .45921 .32371 .14780 .7442 100.94321 .18301 .31930 .97651 .06761 .31851 .52161 .41251 .51851 .49221 .35291 .17780 .7540 90.91431 .16021 .27640 .94091 .02661 .28941 .49971 .41521 .52221 .51101 .36491 .19670 .7617 80.90331 .16041 .27970 .93031 .02531 .29221 .52771 .44891 .57471 .57161 .41681 .24200 .7771 70.89441 .16911 .30660 .92381 .03741 .30811 .58991 .50321 .66381 .66851 .49721 .30040 .7907 60.87311 .16541 .31800 .90611 .03341 .30971 .63681 .54521 .73851 .75771 .56171 .33750 .7933 50.82991 .12901 .28310 .86690 .99281 .27211 .62111 .53081 .73691 .79261 .56561 .32140 .7698 40.74311 .04361 .16590 .78300 .88881 .17001 .46701 .35591 .50641 .66001 .44051 .21400 .7069 $30.59500 .88180 .91520 .64680 .68470 .97971 .11780 .88470 .8781 \quad 1.2335 .1 .10540 .93870 .5926$

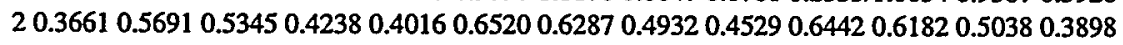

260.31290 .27980 .27660 .26630 .26420 .24790 .24530 .21930 .20540 .18150 .16580 .14250 .1200 250.52540 .45780 .46330 .43820 .44680 .41040 .42150 .36550 .35240 .30740 .29410 .25640 .1980 240.70740 .65180 .68790 .63320 .67010 .59680 .61680 .53510 .53600 .45590 .45120 .37590 .2579 230.81970 .79030 .85250 .77770 .82780 .73570 .74640 .66310 .67500 .57220 .56350 .46240 .3032 220.88330 .86290 .93370 .85630 .90920 .81090 .81890 .73560 .75080 .64120 .62610 .52210 .3448

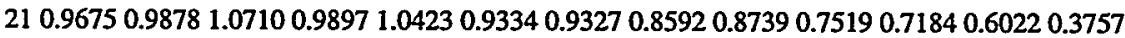
201.02571 .06911 .15681 .07901 .13011 .01801 .02620 .94670 .95810 .83140 .78390 .66550 .4077 191.05991 .10261 .19391 .11941 .17401 .06111 .07660 .99151 .00270 .87350 .81910 .70540 .4394 181.13391 .19061 .27361 .22451 .26821 .16811 .16511 .09631 .08970 .96710 .89140 .76080 .4817 171.17511 .27631 .36661 .34071 .37871 .28901 .27351 .21501 .19441 .07320 .97240 .82450 .5106 161.16801 .29181 .39971 .38661 .43051 .34181 .32791 .26961 .24801 .12511 .01490 .86730 .5352 151.15101 .28181 .40451 .40031 .45141 .36191 .35141 .29401 .27531 .15261 .04040 .89980 .5598 141.16081 .33431 .46511 .47721 .52741 .44781 .44051 .38591 .36221 .24121 .10760 .96560 .5760 131.15941 .34361 .47241 .48891 .54081 .47141 .47381 .42511 .40351 .28661 .14461 .00890 .5932 121.15031 .32221 .43951 .44151 .49691 .44591 .46611 .42661 .41331 .30091 .16001 .03620 .6118 111.14511 .33281 .43191 .42301 .47721 .45751 .49941 .47341 .46441 .35791 .20521 .08480 .6287 101.12951 .31771 .40731 .38071 .44261 .44151 .50051 .49031 .49651 .38881 .23721 .11050 .6365 91.10891 .28821 .38051 .34251 .41731 .41601 .47421 .48771 .51361 .40111 .25971 .11330 .6407 81.09871 .29301 .38941 .35321 .43741 .43961 .50861 .53161 .57281 .45661 .31081 .15220 .6496 71.08241 .31531 .41721 .39101 .48691 .49481 .57991 .60971 .66811 .54501 .38641 .20380 .6540 61.05251 .32191 .43241 .41631 .52911 .53681 .63701 .67281 .75491 .62021 .45021 .22880 .6475 51.00511 .29041 .41331 .39821 .53341 .52671 .62971 .67161 .78751 .63401 .46931 .19010 .6205 40.94001 .19231 .33011 .29041 .43931 .40641 .47571 .52111 .65531 .50321 .38661 .04920 .5649 30.83250 .96531 .09761 .02241 .12821 .10221 .10581 .15601 .23291 .14221 .09410 .76990 .4692 20.58580 .58040 .61020 .59670 .62010 .63800 .61450 .64420 .64460 .62620 .57220 .41990 .3030

260.25820 .27360 .24650 .26410 .23270 .23910 .21210 .21000 .17980 .16530 .13860 .11350 .0971 250.42410 .46530 .40700 .43990 .38650 .41430 .35510 .36810 .30490 .29320 .24000 .20020 .1686 
240.60130 .63880 .58790 .60330 .56360 .58740 .52010 .52850 .45060 .45000 .35410 .29490 .2195 230.72570 .74770 .72070 .71600 .69700 .70170 .64480 .63540 .56400 .56230 .44540 .37300 .2581 220.79060 .80840 .79260 .78510 .77140 .76670 .71470 .69790 .63150 .62500 .50490 .43000 .2940 210.90260 .89540 .91540 .88250 .89690 .86260 .82590 .78950 .73920 .71800 .59050 .50720 .3183 200.97780 .95740 .99860 .95350 .98360 .93160 .90520 .85620 .81690 .78390 .65540 .56890 .3445 191.01290 .99431 .03730 .99661 .02600 .97250 .94820 .89600 .85880 .81960 .69400 .60740 .3721 181.10031 .07941 .13511 .09281 .12951 .06641 .04780 .98450 .95010 .89250 .76610 .64710 .4053 171.18961 .15511 .24191 .18471 .24571 .16101 .15991 .07461 .05260 .97390 .84440 .69860 .4258 161.21451 .18221 .28291 .22611 .29591 .20501 .21201 .11931 .10351 .01660 .88950 .73940 .4457 151.21571 .19131 .29361 .24571 .31481 .22651 .23591 .14441 .13101 .04190 .92160 .77410 .4674 141.27141 .22561 .36611 .29461 .39751 .28411 .32241 .20581 .21621 .10960 .99340 .83570 .4773 131.28741 .23401 .38691 .30741 .42351 .30401 .35921 .23581 .26011 .14661 .03900 .87980 .4904 121.27751 .22521 .37101 .29301 .40771 .29661 .36021 .24301 .27461 .16151 .06540 .91050 .5059 111.29951 .23001 .39261 .29371 .42931 .31241 .40281 .27641 .32911 .20711 .11610 .95190 .5139 101.29501 .22241 .38681 .28541 .42581 .31571 .41701 .29711 .35841 .23991 .14100 .96070 .5138 91.27231 .20641 .36551 .27341 .40861 .31411 .41301 .31011 .37011 .26441 .14800 .93750 .5090 81.27881 .20711 .38411 .28911 .43681 .34231 .45301 .35151 .42201 .31751 .18670 .95460 .5054 $71.29941 .21321 .42861 .32231 .4967^{-1} 1.39171 .52501 .41361 .50271 .39561 .24080 .97640 .4952$ 61.30381 .20801 .46181 .34981 .54731 .43491 .58421 .46711 .56841 .46421 .27130 .97270 .4776 51.27401 .18191 .45401 .35091 .55581 .44631 .59041 .48191 .57591 .48961 .24090 .91970 .4459 41.18541 .12261 .35671 .29191 .46101 .38391 .47661 .41001 .45551 .41061 .10950 .78890 .3954 30.97510 .98671 .08791 .10581 .16111 .16671 .15211 .16631 .12071 .10800 .83750 .58260 .3282 20.59930 .67040 .64130 .71360 .67500 .73170 .66080 .69990 .62680 .57810 .46440 .32450 .2175

260.25400 .22700 .22710 .21490 .21030 .19710 .19060 .17000 .15780 .14160 .11320 .1020 250.42250 .37600 .38830 .35930 .36020 .33220 .33180 .28900 .27730 .25460 .19970 .1641 240.56680 .53930 .58080 .52470 .54550 .48890 .50250 .42540 .42210 .37450 .29430 .2129 230.65960 .65760 .71270 .64930 .68890 .60910 .62350 .53180 .53000 .46070 .37380 .2540 220.71500 .72170 .78200 .71940 .76590 .67870 .69300 .59630 .59430 .51990 .43080 .2946 210.78770 .82930 .89230 .83560 .89060 .79180 .79830 .69630 .68470 .59620 .50510 .3283 200.84270 .90490 .96820 .91650 .97430 .87180 .87530 .76880 .75060 .66030 .56600 .3665 190.88070 .94351 .00840 .95691 .01630 .91300 .91960 .80890 .79170 .70180 .60500 .4028 180.96281 .03501 .09151 .05301 .09661 .00751 .00100 .89380 .85780 .75820 .64520 .4427 171.03681 .13441 .18741 .16031 .19541 .11351 .09490 .98810 .93260 .82240 .69700 .4718 161.08011 .17501 .23771 .20761 .24401 .16291 .14561 .03580 .97760 .86530 .73790 .4971 $151.10071 .19031 .25731 .22671 .26601 .1859^{\circ} 1.17411 .06291 .00830 .89750 .77270 .5225$ 141.13861 .26061 .33071 .30571 .34311 .26821 .25211 .14091 .07410 .96270 .83440 .5436 131.15491 .28781 .35951 .33631 .37371 .30431 .29111 .18191 .11331 .00530 .87850 .5640 121.15811 .28581 .35751 .33281 .37271 .30751 .30281 .19661 .13541 .03210 .90900 .5823 111.17251 .31911 .39111 .36871 .40661 .35101 .34841 .24641 .18081 .07940 .95040 .5936 101.17711 .32561 .40311 .37771 .42101 .36891 .37581 .27281 .21261 .10630 .95930 .5958 91.17421 .31331 .39621 .36981 .42321 .37021 .38971 .28281 .23451 .11480 .93640 .5921 81.18611 .33491 .42891 .40191 .46111 .41291 .44021 .32921 .28091 .15740 .95460 .5956 71.20251 .37791 .49171 .46221 .52431 .48621 .51991 .39991 .34091 .21430 .97740 .5982 61.20711 .40671 .54691 .51261 .57891 .54981 .59071 .45351 .38091 .24950 .97620 .5909 51.18881 .39461 .56111 .52081 .60141 .56811 .61771 .45181 .37111 .22640 .92690 .5633 41.13381 .29961 .46111 .42771 .53861 .47481 .53031 .33891 .27421 .10190 .79960 .5028 30.99451 .04761 .13021 .13571 .25271 .16321 .19731 .04111 .01110 .81450 .59250 .4005 20.66730 .62410 .63580 .66160 .68390 .66520 .64430 .59000 .54100 .43870 .32990 .2317

260.19990 .21780 .19450 .19850 .17990 .17640 .15490 .14100 .14230 .11920 .0967 250.33300 .36540 .32790 .35180 .30660 .31460 .26590 .24640 .23730 .19670 .1674 240.47710 .49600 .47720 .51630 .45260 .47270 .39210 .36980 .31530 .25690 .2181

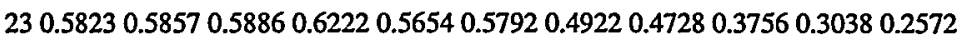
220.64180 .64410 .65180 .68210 .63170 .64020 .55510 .53990 .42650 .34610 .2932 $\begin{array}{llllllllllllll}21 & 0.7394 & 0.7291 & 0.7521 & 0.7682 & 0.7349 & 0.7287 & 0.6482 & 0.6343 & 0.4688 & 0.3772 & 0.3183\end{array}$ 200.80960 .79330 .82410 .82960 .80810 .79060 .71610 .70030 .51260 .41110 .3482 $\begin{array}{lllllllllllll}19 & 0.8490 & 0.8351 & 0.8628 & 0.8652 & 0.8462 & 0.8240 & 0.7538 & 0.7380 & 0.5498 & 0.4445 & 0.3770\end{array}$ 180.93590 .91750 .94930 .94210 .93080 .89550 .83000 .78900 .60240 .48780 .4104 171.03060 .99821 .04411 .02051 .02440 .97140 .91350 .85340 .64120 .51780 .4318 161.07511 .03931 .08741 .05931 .06911 .00880 .95720 .89500 .67090 .54290 .4521 151.09701 .06331 .10891 .08051 .09181 .02930 .98370 .92380 .69840 .56770 .4737 
141.16741 .11541 .18081 .13221 .16561 .08481 .05400 .98620 .72050 .58460 .4847 131.20031 .14141 .21361 .15561 .20061 .11191 .09251 .02280 .74020 .60210 .4982 121.20781 .14891 .21961 .16021 .20881 .11961 .10911 .04310 .75850 .62050 .5135 111.24701 .17481 .25911 .18551 .25241 .15391 .15531 .08370 .77290 .63790 .5221 101.26071 .18691 .27431 .19991 .27371 .18001 .18031 .10600 .78300 .64620 .5229 91.25591 .18871 .27201 .20591 .27941 .20071 .19091 .11530 .79060 .65080 .5191 81.28251 .21031 .30301 .23261 .32111 .24861 .23511 .15600 .80580 .66010 .5167 71.32771 .24481 .35531 .27281 .38681 .31971 .30001 .20780 .81860 .66490 .5079 61.35651 .26811 .39361 .30431 .43991 .38491 .34741 .23830 .82050 .65930 .4918 51.34051 .26071 .38811 .31021 .44841 .41811 .34041 .21950 .79840 .63430 .4626 41.24141 .19661 .29201 .25861 .35641 .36501 .22741 .11410 .73710 .58210 .4128 30.99181 .01551 .02841 .07321 .07251 .12080 .94560 .84600 .62500 .48840 .3425 20.58410 .62190 .59840 .65790 .61250 .60740 .53180 .45830 .41380 .31620 .2228

260.17230 .16530 .16990 .15970 .15240 .13910 .12310 .1229 250.30260 .28100 .30320 .28120 .27050 .24710 .21790 .2134 240.44560 .40610 .44700 .42510 .41130 .37570 .32610 .2844 230.54220 .50010 .53870 .53630 .51460 .47910 .41740 .3387 220.60120 .55650 .59090 .59920 .57580 .54210 .47950 .3850 210.68700 .64190 .65980 .68830 .65720 .62640 .56300 .4190 200.75360 .70510 .71250 .75070 .71650 .68620 .62360 .4508 190.79700 .74270 .74580 .78640 .75340 .72090 .65800 .4806 180.86790 .81870 .80960 .84120 .81000 .77070 .69780 .5215 170.94680 .90040 .87370 .90580 .87250 .82910 .74900 .5492 160.99310 .94310 .90870 .94400 .91020 .86580 .78530 .5722 151.02300 .96850 .93070 .96840 .93590 .89170 .81260 .5947 141.08891 .03250 .97521 .02330 .98830 .94470 .86590 .6076 131.12671 .06690 .99861 .05281 .01840 .97640 .89920 .6208 121.14501 .08101 .00851 .06711 .03530 .99530 .92030 .6347 111.18591 .11981 .03361 .10161 .07051 .03240 .95520 .6413 101.20861 .13541 .04791 .12361 .09521 .05880 .97170 .6451 91.21611 .13531 .05531 .13701 .11361 .07820 .97460 .6482 81.25081 .16221 .07831 .17241 .15161 .12091 .00830 .6564 71.30091 .20131 .10761 .21571 .19571 .17331 .04870 .6588 61.33231 .22151 .12501 .24251 .22251 .20881 .06890 .6514 51.31431 .19561 .11601 .23361 .21271 .20141 .04460 .6266 41.20891 .09121 .05911 .15811 .13751 .10910 .93180 .5744 30.94370 .85600 .89400 .92140 .89430 .84620 .68890 .4802 20.50790 .49480 .51750 .50450 .48160 .45290 .37850 .3156

260.13050 .12990 .14410 .13350 .12620 .11270 .1014 250.22500 .22810 .23910 .23430 .20900 .19080 .1787 240.32180 .33460 .31350 .31180 .27340 .25100 .2355 230.39650 .42080 .36840 .36660 .32190 .29710 .2789 220.44570 .47790 .41410 .40990 .36360 .33740 .3173 $210.51070 .55290 .45270 .44200 .39560 .3707 \quad 0.3421$ 200.56190 .61070 .48890 .47160 .43190 .40660 .3678

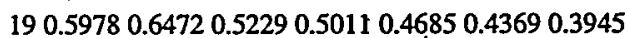
180.66090 .68950 .57090 .54250 .51020 .47380 .4269 170.72500 .74220 .60510 .56980 .53840 .49880 .4456 160.76370 .78020 .63030 .59140 .56040 .51960 .4633 150.79220 .80880 .65250 .61180 .58070 .53970 .4819 140.84250 .85820 .66750 .62090 .59370 .55210 .4879 130.87470 .88910 .68020 .63040 .60580 .56440 .4967 120.89350 .90750 .69100 .64090 .61790 .57720 .5074 110.92320 .93510 .69720 .64370 .62630 .58430 .5086 100.93410 .94340 .69900 .64470 .63250 .58970 .5086 90.93180 .93320 .69870 .64570 .63830 .59490 .5081 80.94600 .94740 .70360 .64830 .64820 .60290 .5085 70.96230 .96400 .70450 .64210 .65300 .60460 .5011

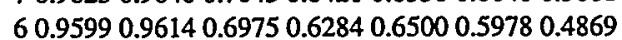
50.92100 .92030 .67540 .60550 .63450 .57710 .4631 
40.81490 .80580 .63230 .56960 .59710 .53490 .4210 30.62470 .60500 .54840 .50160 .51020 .45400 .3567 $\begin{array}{lllllllll}2 & 0.3578 & 0.3431 & 0.3664 & 0.3466 & 0.3243 & 0.2936 & 0.2393\end{array}$

260.10140 .0972

250.16850 .1760

240.21540 .2322

230.24980 .2704

220.28030 .3012

210.29850 .3223

200.32290 .3430

190.34990 .3643

180.38420 .3945

170.40760 .4137

160.42740 .4293

150.44610 .4440

140.45580 .4485

130.46600 .4535

120.47620 .4582

110.47900 .4544

100.47790 .4475

90.47400 .4383

80.47140 .4302

70.46430 .4178

60.45210 .4026

50.42800 .3788

40.38800 .3473

30.32660 .3009

20.21140 .2068

EDIT: IPROPT[19] $=2$

Bundle Average Fast Group Fluxes (NEM / Reference LP) *1.0E-14

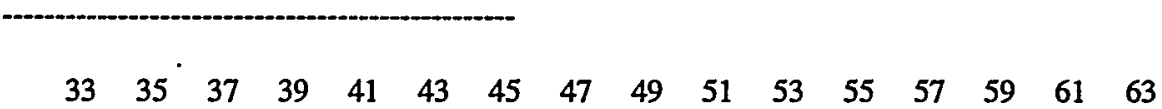

32- 2.48922 .83812 .83082 .45802 .44482 .77632 .68522 .15771 .96872 .11982 .12972 .06301 .93901 .73951 .23520 .6523 30- 2.87372 .80112 .76482 .67542 .68122 .73732 .68572 .45642 .30542 .26552 .23572 .13251 .96741 .64521 .18770 .5702 28- 2.85842 .77432 .80302 .74512 .72932 .80762 .78422 .62982 .43652 .44552 .33292 .25541 .97161 .60411 .0256 $26-2.44612 .67022 .74362 .31932 .32442 .66952 .70732 .44762 .34012 .42762 .38262 .23991 .99401 .57780 .9252$ 24- 2.40032 .65402 .71702 .31992 .36572 .73712 .76732 .52562 .36472 .51062 .39812 .28641 .95641 .52680 .8634 22- 2.69952 .69082 .78082 .65682 .73272 .70692 .71062 .63162 .54222 .48922 .38692 .19151 .90611 .44830 .7898 20- 2.60482 .62242 .73982 .68282 .75812 .70942 .69312 .72652 .59082 .54062 .32782 .13471 .72551 .23460 .6168 18- 2.10562 .38462 .55712 .40812 .50312 .62712 .72362 .61302 .54642 .44132 .23931 .91761 .48770 .8567 16- 1.93272 .25902 .37792 .3031 .2 .33672 .52282 .57762 .52922 .45242 .39642 .10431 .74361 .1398 14- 2.09762 .23832 .41592 .40352 .49202 .46292 .51192 .43132 .39752 .18931 .93831 .51230 .8807 $12-2.11232 .21622 .31912 .37222 .39182 .37982 .31762 .24632 .11661 .94711 .59291 .17000 .6144$ 10- 2.05462 .12242 .25372 .24432 .29612 .20322 .14901 .94391 .77481 .52881 .17480 .7245 08- 1.93171 .96121 .97812 .01551 .98411 .93391 .75001 .51611 .16760 .89650 .6231 06- 1.73591 .64561 .61741 .61831 .57941 .48561 .26380 .8761

04- 1.23231 .18931 .03700 .95510 .90900 .82510 .6370

02- 0.65080 .5717

Bundle Average Thermal Group Fluxes (NEM/Reference LP) *1.0E-13

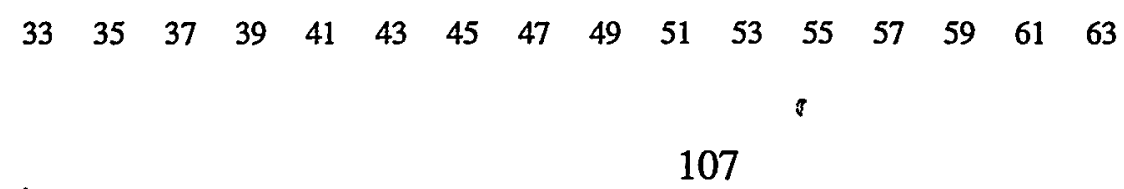


32- 4.41135 .17025 .26873 .95583 .92775 .22545 .10784 .23573 .47774 .76954 .11004 .62303 .80263 .69962 .69071 .8533 30- 5.37615 .00535 .71304 .83035 .44704 .88635 .45264 .57175 .00984 .29254 .83924 .08424 .33823 .35742 .76151 .9045 28-5.3410 5.7183 5.1214 5.1902 5.1415 5.2216 5.1979 5.0140 4.5112 4.7555 4.3453 4.43393.8489 3.6969 2.7478 26- 3.91134 .83475 .18853 .68454 .23034 .83285 .61474 .20324 .61384 .49325 .09994 .21644 .41493 .40492 .5730 24- 3.8351 5.4906 5.1334 4.2233 3.8662 5.1683 5.1959 4.5046 4.0833 4.8481 4.4349 4.5186 3.81473.15302.3891 22- 4.94774 .82815 .18364 .81825 .15414 .79855 .46274 .74515 .27314 .53705 .09394 .14224 .20943 .20042 .1917 20-4.9714 5.3392 5.1307 5.5610 5.1744 5.3162 4.7684 5.1278 4.6683 4.7587 4.3152 4.2978 3.4642 2.8246 1.9618 $18-4.15404 .42734 .66554 .13594 .36754 .75065 .12074 .65505 .14144 .47734 .84173 .71803 .27032 .5864$ 16 - 3.4357 4.8595 4.3853 4.5699 4.03175.3615 4.6743 5.2900 4.4412 4.6590 4.0178 3.5675 2.9768 $14-4.72024 .26134 .71724 .46254 .82254 .47954 .58444 .46834 .66054 .11614 .18963 .22132 .4473$ $12-4.09034 .88124 .33585 .09294 .42635 .06244 .27554 .83834 .03494 .19553 .27322 .67961 .9920$ $10-4.60334 .07244 .43414 .22094 .53094 .15614 .31863 .77503 .73843 .25032 .68192 .1251$ 08- 3.7962 4.3465 3.8577 4.45103.8548 4.2414 3.4974 3.32473.0771 2.4750 1.9872 06- 3.69653 .35813 .71453 .49953 .42063 .25732 .88322 .5566 $04-2.68662 .76052 .76762 .63522 .44542 .25132 .0556$ $02-1.78681 .9052$

EDIT: $\operatorname{IPROPT}[40]=1$ Bundle Peak kW/ft (MLHGR) Map (NEM / Reference LP)

\section{$\begin{array}{llllllllllllllll}33 & 35 & 37 & 39 & 41 & 43 & 45 & 47 & 49 & 51 & 53 & 55 & 57 & 59 & 61 & 63\end{array}$}

$32-9.817411 .24511 .27010 .0799 .822611 .18910 .6318 .040710 .5607 .06129 .09667 .56469 .50829 .04756 .69522 .9674$ 30- 11.434 11.649 8.5694 10.8809.196011.274 8.8862 10.596 7.6431 9.4310 7.64079.8433 8.3508 8.49996.4769 2.6784 28- 11.4368 .596011 .09110 .75511 .24111 .53911 .1449 .677610 .3579 .470510 .20710 .5049 .66207 .17794 .3375 26- 9.874610 .80910 .71710 .8487 .990310 .7568 .472811 .5189 .032410 .4588 .641910 .5128 .20177 .88113 .7940 24-9.7049 8.8952 11.132 8.05469.7739 10.914 10.898 10.81511.510 10.406 10.844 10.541 9.82767.83473.5768 22- 10.68810 .93911 .32810 .67610 .81510 .8518 .795711 .0678 .588810 .9299 .147110 .7988 .93627 .75563 .5077 $20-10.1318 .561610 .8998 .496710 .8098 .867411 .15111 .36611 .52211 .65411 .21610 .8409 .21066 .94192 .9923$ $18-7.951810 .2409 .676311 .38510 .77911 .10711 .35911 .96710 .39511 .7959 .38939 .95338 .11313 .9539$ 16- 10.3597 .465010 .0929 .009911 .4028 .560411 .4189 .683212 .11411 .99110 .8778 .94144 .9255 14- 6.9935 9.38809.412510.421 10.237 10.708 11.16311.674 11.957 11.3249.66078.3028 4.0679 $12-9.06547 .509610 .1758 .493410 .7889 .165411 .0459 .446210 .9279 .81918 .80566 .46433 .1191$ $10-7.51869 .780510 .49710 .53810 .59910 .86710 .79510 .1069 .09508 .43786 .46143 .8380$ 08- 9.41968 .08479 .68438 .331110 .0239 .24939 .34968 .26435 .07254 .13513 .2111 06- 8.8571 8.4594 7.24168.2533 8.0688 8.0530 7.1710 4.0699 $04-6.65736 .45344 .38383 .94474 .09343 .76123 .1094$ 02- 2.97082 .6944

EDIT: IPROPT[40] $=1$

Bundle MFLPD Map

(NEM / Reference LP)

$\begin{array}{llllllllllllllll}33 & 35 & 37 & 39 & 41 & 43 & 45 & 47 & 49 & 51 & 53 & 55 & 57 & 59 & 61 & 63\end{array}$

32- 0.75750 .86770 .86960 .77770 .75790 .86330 .82030 .62040 .81480 .54480 .70190 .58370 .73370 .69810 .51660 .2290 $30-0.88220 .89880 .66120 .83950 .70960 .86990 .68570 .81760 .58970 .72770 .58960 .75950 .64440 .65590 .49980 .2067$ 28- $0.88240 .66330 .85580 .82980 .86740 .89040 .8598 \quad 0.74670 .79920 .73070 .78760 .81050 .74550 .55380 .3347$ 26- 0.76190 .83400 .82690 .83700 .61650 .82990 .65380 .88880 .69690 .80700 .66680 .81110 .63290 .60810 .2927 24- 0.74880 .68640 .85890 .62150 .75420 .84210 .84090 .83450 .88810 .80300 .83670 .81330 .75830 .60450 .2760 22- 0.82470 .84400 .87410 .82380 .83450 .83730 .67870 .85390 .66270 .84330 .70580 .83320 .68950 .59840 .2707 20- 0.78170 .66060 .84100 .65560 .83400 .68420 .86040 .87700 .88900 .89920 .86540 .83640 .71070 .53560 .2309 $18-0.61360 .79010 .74660 .87840 .83170 .85710 .87650 .92330 .80210 .91010 .72450 .76800 .62600 .3051$ $16-0.79930 .57600 .77870 .69520 .87980 .66050 .88100 .74720 .93470 .92530 .83930 .68990 .3801$ 14- 0.53960 .72440 .72630 .80410 .78990 .82620 .86130 .90080 .92260 .87380 .74540 .64060 .3139 
$12-0.69950 .57940 .78510 .65540 .83240 .70720 .85230 .72890 .84320 .75770 .67940 .49880 .2407$

$10-0.58010 .75470 .81000 .81310 .81780 .83850 .83300 .77980 .70180 .65110 .49860 .2961$

$08-0.72680 .62380 .74720 .64280 .77340 .71370 .72140 .63770 .39140 .31910 .2478$

$06-0.68340 .65270 .55880 .63680 .62260 .62140 .55330 .3140$

$04-0.51370 .49790 .33830 .30440 .31590 .29020 .2399$

02- 0.22920 .2079

Bundle CPR Map

(NEM / Reference LP)

$\begin{array}{llllllllllllllll}33 & 35 & 37 & 39 & 41 & 43 & 45 & 47 & 49 & 51 & 53 & 55 & 57 & 59 & 61 & 63\end{array}$

32- 1.82861 .59251 .57471 .98251 .98241 .61191 .61762 .01211 .86382 .04081 .88642 .23772 .13942 .48413 .15694 .4843 30- 1.54931 .49201 .69481 .55871 .79961 .54031 .75731 .62001 .91541 .76732 .04911 .94722 .33822 .47813 .31314 .5743 28- 1.55221 .68921 .60241 .61381 .61851 .60611 .58131 .63151 .63811 .76901 .78921 .97562 .11222 .73223 .0261 26- 1.99161 .55501 .61131 .87912 .08821 .54881 .71601 .57121 .85831 .68131 .98561 .88962 .30142 .68603 .2627 24-2.0157 1.8009 1.61712.0833 1.96971.6219 1.5709 1.6381 1.64341.75431.76771.9541 2.1471 2.8583 3.5207 22- 1.67301 .55681 .61261 .55241 .62021 .55861 .76051 .57561 .85791 .70632 .00201 .95092 .40592 .90473 .7768 20- 1.65991 .79021 .59951 .72291 .57471 .77311 .58531 .65861 .65841 .80721 .85282 .10542 .41923 .26904 .2793 $18-2.04731 .67521 .72001 .60241 .67271 .57381 .65601 .66961 .93321 .77932 .13942 .22412 .81943 .3253$ 16- 1.90381 .98211 .68811 .88201 .66631 .84701 .65401 .93681 .79591 .90962 .04292 .56012 .8500 $14-2.06451 .78811 .78801 .69551 .75941 .72371 .84651 .77991 .90471 .98722 .36102 .85153 .4786$ 12- 1.89942 .06061 .79621 .99011 .77142 .00241 .86612 .12882 .03332 .35322 .58963 .41884 .3063 $10-2.24081 .95321 .97381 .88861 .94771 .94442 .08992 .18992 .47802 .84033 .41773 .8624$ $08-2.14102 .34572 .10742 .28612 .12802 .38312 .39582 .77452 .75873 .42914 .2518$

06- 2.4819 2.4741 2.71752.6204 2.7171 2.85863.2108 3.2985

04- 3.15853 .30753 .00633 .19713 .37003 .64624 .1509

$02-4.53924 .5698$

Bundle R-Factors

$\begin{array}{llllllllllllllll}33 & 35 & 37 & 39 & 41 & 43 & 45 & 47 & 49 & 51 & 53 & 55 & 57 & 59 & 61 & 63\end{array}$

32- 1.01101 .00281 .00271 .00301 .00271 .00351 .00271 .01491 .04071 .01451 .04071 .01431 .04071 .00291 .04071 .0151 30- 1.00311 .04071 .01441 .04071 .01391 .04071 .01301 .04071 .01401 .04071 .01221 .04071 .01041 .04071 .00511 .0152 28- 1.00271 .01401 .00271 .00321 .00271 .00301 .00271 .00291 .04071 .00281 .04071 .00281 .04071 .00801 .0145 26- 1.00281 .04071 .00311 .04071 .01431 .04071 .01411 .04071 .01371 .04071 .01281 .04071 .00971 .00281 .0156 24- 1.00271 .01441 .00261 .01411 .00661 .00351 .00261 .00311 .04071 .00301 .04071 .00281 .04071 .00271 .0156 22- 1.00281 .04071 .00281 .04071 .00311 .04071 .01321 .04071 .01381 .04071 .01141 .04071 .00801 .00271 .0148 $20-1.00271 .01311 .00261 .01381 .00261 .01241 .04071 .00301 .04071 .00271 .04071 .00321 .04071 .00491 .0152$ 18- 1.01481 .04071 .00271 .04071 .00271 .04071 .00281 .04071 .01061 .04071 .01071 .04071 .00261 .0157 16- 1.04071 .01401 .04071 .01361 .04071 .01431 .04071 .01181 .04071 .00281 .04071 .00281 .0155 14- 1.01431 .04071 .00291 .04071 .00281 .04071 .00271 .04071 .00281 .04071 .00571 .00271 .0154 $12-1.04071 .01281 .04071 .01281 .04071 .01111 .04071 .01051 .04071 .00551 .04071 .00951 .0154$ $10-1.01401 .04071 .00271 .04071 .00281 .04071 .00311 .04071 .00341 .00271 .00961 .0129$ 08- 1.04071 .01081 .04071 .00971 .04071 .00771 .04071 .00261 .01531 .01511 .0145 $06-1.00321 .04071 .00801 .00271 .00321 .00271 .00501 .0150$ 04- 1.04071 .00551 .01461 .01531 .01411 .01391 .0153 $02-1.01421 .0151$

EDIT: IPROPT[45] = I

Bundle FLCPR Map (NEM / Reference LP)

$\begin{array}{llllllllllllllll}33 & 35 & 37 & 39 & 41 & 43 & 45 & 47 & 49 & 51 & 53 & 55 & 57 & 59 & 61 & 63\end{array}$

32- 0.76010 .87290 .88270 .70110 .70120 .86230 .85930 .69080 .74580 .68110 .73690 .62120 .64970 .55960 .44030 .3100 30- 0.89720 .93170 .82020 .89180 .77240 .90240 .79100 .85800 .72570 .78650 .67840 .71380 .59450 .56090 .41960 .3039 
28- 0.89550 .82290 .86750 .86130 .85880 .86550 .87900 .85200 .84860 .78580 .77690 .70360 .65810 .50870 .4593 26- $0.69790 .89390 .86270 .73970 .66560 .89750 .81000 .88470 .74800 .82670 .7000 \quad 0.73560 .60400 .51750 .4260$ 24- 0.68960 .77190 .85960 .66720 .70570 .85700 .88480 .84860 .84580 .79240 .78630 .71130 .64740 .48630 .3948 22- $0.8308 \quad 0.89290 .86200 .89540 .85790 .89180 .78950 .88220 .74820 .81460 .6943 \quad 0.71250 .57770 .47850 .3680$ 20- $0.83740 .77640 .86900 .80680 .88270 .78400 .87680 .83810 .83820 .76910 .7502 \quad 0.66020 .57460 .42520 .3248$

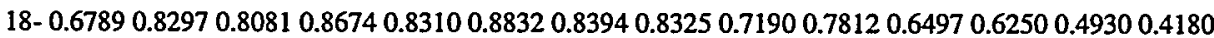

16- 0.73010 .70130 .82340 .73860 .83420 .75260 .84040 .71770 .77400 .72790 .68040 .54290 .4877

$14-0.67330 .77740 .77740 .81980 .79000 .80640 .75280 .78090 .72980 .69950 .58870 .48750 .3996$

$12-0.73180 .67460 .77390 .69850 .78470 .69420 .74490 .65290 .68360 .59070 .53680 .40660 .3228$

$10-0.62030 .71160 .70420 .73600 .71370 .71490 .66510 .63470 .56090 .48940 .40670 .3599$

$08-0.64920 .59260 .65960 .60800 .65320 .58330 .58020 .50100 .50390 .40530 .3269$

$06-0.56000 .56180 .51150 .53050 .51160 .48630 .43290 .4214$

04- 0.44010 .42030 .46240 .43480 .41250 .38120 .3349

02- 0.30620 .3042

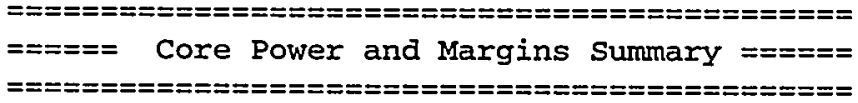

CORE POWER AND FLOW:

Rated Thermal Power (MWt):

Core Thermal Power (MWt):

Percentage Power:

Core Average Exposure (GWD/STU):
3833.000 Rated Coolant Flow Rate $(\mathrm{Mlbm} / \mathrm{hr})$ : 3833.000 Core Coolant Flow Rate $(\mathrm{Mlbm} / \mathrm{hr})$ : 100.000 Percentage Core Flow:

13.7783 Numbex Fuel Assemblies:
112.500

93.752

83.335

800

13.58

12.733

0.000
Average Linear Heat Rate (kW/ft)
Average Heat Flux (MBtu/hr-ft2): Average Power Density $(\mathrm{kW} / \mathrm{l})$ : Bypass Exit Void:

T/H CONDITIONS

Inlet Enthalpy (Btu/lbm)

Inlet Temperature $(F)$ :

Average Coolant Temperature (F):

Average Fuel Temperature (F):

PRESSURE DROP:

Core Inlet Pressure (psia):

Core Exit Pressure (psia):

Steam Dome Pressure (psia):

LIMITS :

Peak Core Rel. Power (TPF): Peak Average Nodal Rel. Power: Max. Ass. Axial Peaking (APF): Max. Ass. Rel, Power (RPF): Core MLHGR (CMRPD) : Core MFLPD (CMFLPD) : Core MAPLHGR (CLHGR) : Core MAPRAT (CMAPR): Core MCPR:

Core MFLCPR (CMFCP) :

Eighth Core Power Imbalance:
5.2662 Bypass and WT Flow Fraction $(8)$ : 0.1557 Total Bypass Flow Rate $(\mathrm{M} l \mathrm{bm} / \mathrm{hr})$
54.1418 Water Tube Flow Rate $(\mathrm{Mblm} / \mathrm{hr})$ : 0.0000
532.6852 Inlet Subcooling (Btu/lbm): 537.00 Avg. Coolant Void Fraction: 550.88 Avg. Exit Void Fraction: 959.00 Avg. Coolant Density (g/cc):

1077.62 Core Support Plate DEL-P (psi): 1058.28 Required Core Pres. Drop (psi): 1047.00 Average Bundle Press. Drop (psi):
21.5000

0.5332

0.8001

0.3744

1077.62 19.34 19.33 


\section{INTERNAL DISTRIBUTION}

1. M. D. DeHart

2. F. C. Difilippo

3.. R. J. Ellis

4. J. C. Gehin

5. S. R. Greene

6. D. T. Ingersoll

7. M. A. Kuliasha

8. . G. E. Michaels
9-13. R. T. Primm III

14. C. C. Southmayd

15-19. D. J. Spellman

20. Central Research Library

21. FMDP Library

22-23. ORNL Laboratory Records (OSTI)

24. ORNL Laboratory Records-RC

\section{EXTERNAL DISTRIBUTION}

25. M. L. Adams, Department of Nuclear Engineering, Texas A\&M University, Zachry 129, College Station, TX 77843

26-28. Imelda Ariani, Department of Nuclear Engineering, P.O: Box 7909, North Carolina State University, Raleigh, NC 27695-7909

29. L. Holgate, Office of Fissile Materials Disposition, U.S. Department of Energy, MD-1/2, 1000 Independence Avenue SW, Washington DC 20585

30. G. S. Chang, INEEL, P.O. Box 1625, MS-3885, Idaho Falls, ID 83415-3885

31. D. Nulton, Office of Fissile Materials Disposition, U.S. Department of Energy, MD-4, 1000 Independence Avenue SW, Washington, DC 20585

32-36. Office of the ORNL Site Manager, Department of Energy, Oak Ridge National Laboratory, P.O. Box 2008, Oak Ridge, TN 37831

37. P. T. Rhoads, Office of Fissile Materials Disposition, U.S. Department of Energy, MD-4, 1000 Independence Avenue SW, Washington, DC 20585

38. J. Thompson, Office of Fissile Materials Disposition, U.S. Department of Energy, MD-4, 1000 Independence Avenue SW, Forrestal Building 3F043, Washington, DC 20585

39-48. Dr. Paul Turinsky, Department of Nuclear Engineering, North Carolina State University, Raleigh, NC 27695-7909

49-51. Dr. Brian Moore, GE Nuclear Energy, P.O. Box 780, Wilmington, NC 28402-0780 